University of Louisville

ThinkIR: The University of Louisville's Institutional Repository

Electronic Theses and Dissertations

8-2018

\title{
Modeling and validations of control parameters for material extrusion-based additive manufacturing of thixotropic aluminum alloys.
}

Lars Herhold

University of Louisville

Follow this and additional works at: https://ir.library.louisville.edu/etd

Part of the Manufacturing Commons, Mechanics of Materials Commons, Metallurgy Commons, and the Other Mechanical Engineering Commons

\section{Recommended Citation}

Herhold, Lars, "Modeling and validations of control parameters for material extrusion-based additive manufacturing of thixotropic aluminum alloys." (2018). Electronic Theses and Dissertations. Paper 3055.

https://doi.org/10.18297/etd/3055

This Doctoral Dissertation is brought to you for free and open access by ThinkIR: The University of Louisville's Institutional Repository. It has been accepted for inclusion in Electronic Theses and Dissertations by an authorized administrator of ThinkIR: The University of Louisville's Institutional Repository. This title appears here courtesy of the author, who has retained all other copyrights. For more information, please contact thinkir@louisville.edu. 


\title{
MODELING AND VALIDATION OF CONTROL PARAMETERS FOR MATERIAL EXTRUSION-BASED ADDITIVE MANUFACTURING OF THIXOTROPIC ALUMINUM ALLOYS
}

\author{
By \\ Lars Herhold \\ Dipl.Ing.(FH) University of Applied Science Berlin, 2001 \\ Master of Science, University of Louisville, 2010
}

\author{
A Dissertation \\ Submitted to the Faculty of the \\ J. B. School of Engineering of the University of Louisville \\ in Partial Fulfillment of the Requirements for the \\ Doctor of Philosophy \\ in Industrial Engineering \\ Department of Industrial Engineering \\ Speed School of Engineering \\ University of Louisville \\ Louisville, Kentucky, USA
}

August 2018 
Copyright 2018 by Lars Herhold

All rights reserved 



\title{
MODELING AND VALIDATION OF CONTROL PARAMETERS FOR MATERIAL EXTRUSION-BASED ADDITIVE MANUFACTURING OF THIXOTROPIC ALUMINUM ALLOYS
}

\author{
By \\ Lars Herhold \\ Dipl.Ing.(FH) University of Applied Science Berlin, 2001 \\ Master of Science, University of Louisville, 2010
}

A Dissertation approved on

May 22, 2018

By the following Dissertation Committee:

Dissertation Director: Dr. Brent Stucker

Dr. Gail W. DePuy

Dr. Thomas L. Starr

Dr. Kevin Chou 


\title{
DEDICATION
}

This dissertation is dedicated to my parents

\author{
Mrs. Karin Herhold \\ and \\ Mr. Rolf Herhold
}

who supported me during the years of work.

This dissertation is also dedicated to my loved son

Mr. Jasper Hannes Sommerfeldt 


\section{ACKNOWLEDGMENTS}

I would like to thank my major professor, Dr. Brent Stucker for his guidance and endurance. I would also thank Rene Vetter for his laboratory support and David Belau for his support in the lab equipment production. Also, many thanks to everyone from FZS Berlin who supported me with ideas and analysis help like Dr. Sven Gall, Dr. Soeren Mueller and Stefan Kuhnke. Furthermore, I would like to thank Tobias Norkowski for his support during the study course and joint work at the beginning of this project. 


\begin{abstract}
MODELING AND VALIDATION OF CONTROL PARAMETERS FOR MATERIAL EXTRUSION-BASED ADDITIVE MANUFACTURING OF THIXOTROPIC

ALUMINUM ALLOYS
\end{abstract}

Lars Herhold

May 22, 2018

Additive Manufacturing (AM) with metals has been accomplished mainly through powder bed fusion processes. Initial experiments and simulations using Material Extrusion Additive Manufacturing (MEAM) have been performed by various researchers especially using low melting alloys. Recently Stratasys Inc. submitted a patent application for the use of their Material Extrusion technology also called Fused Deposition Modeling (FDM) where they describe the process using thixotropic semi-solid alloys. Currently this process using semi-solid, engineering type alloys such as A356 or THIXALLOY 540 aluminum have not been researched to evaluate the control parameters. This research combines the in-depth knowledge of applying thixotropic semi-solid aluminum alloy processing as used in thixocasting and thixoforming with the MEAM research. Successful implementation of this metal AM process category besides powder bed fusion would result in the gain of certain MEAM process advantages like speed and ease of material handling (filament) for metal AM.

In this dissertation thixotropic aluminum alloys have been identified for their applicability for MEAM and optimal pre-processing as well as thermo-mechanical handling in a nozzle has been identified. A review of the optimal heating temperature for an aligned quality of microstructure were completed to provide experimental proof of thixotropic aluminum alloy applicability. As further research aging of such alloys during isothermal holding while pausing or pure movement of 
a MEAM nozzle will help to derive the required cleaning processes in case the alloy develops an in-adequate microstructure. The research results build the basis for the next phases towards a larger project goal of developing a successful MEAM machine for producing aluminum alloy parts. 


\section{TABLE OF CONTENTS}

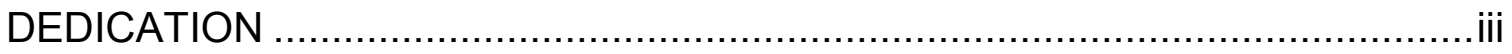

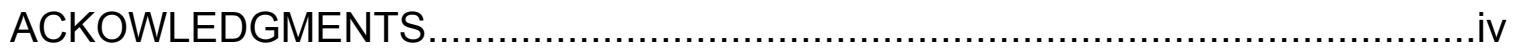

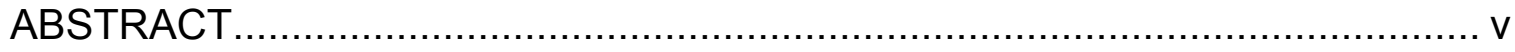

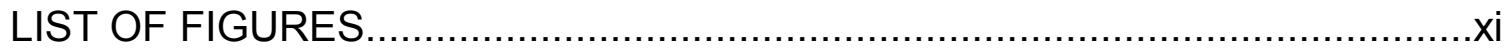

LIST OF TABLES....................................................................................

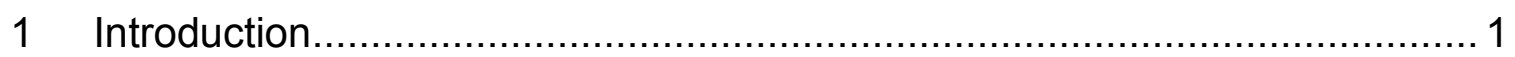

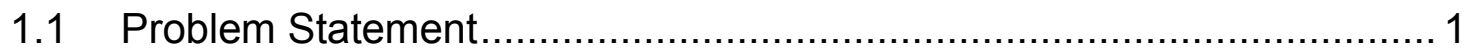

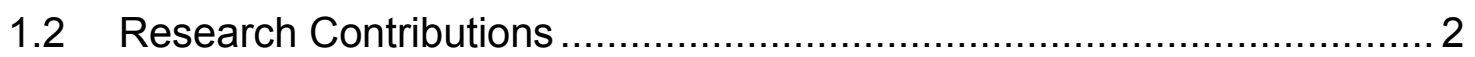

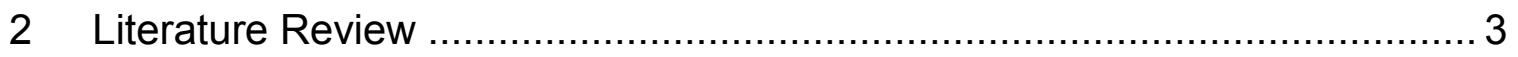

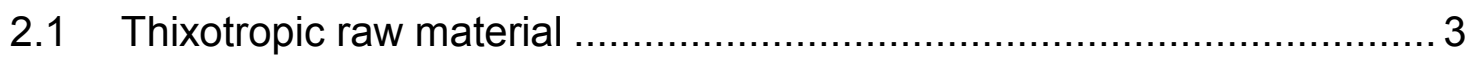

2.1.1 Fundamentals of aluminum alloys............................................. 3

2.1.2 Fundamentals of thixotropic alloy behavior ................................ 7

2.1.3 Semi-solid states of thixotropic alloys ….................................. 8

2.1.4 Shape and structure of thixotropic alloy particles......................... 10

2.1.5 Viscosity, Shear and plastic behavior of semi-solid aluminum alloys 13

2.1.6 Temperature and energetic behavior of thixotropic alloys.............. 17

2.1.7 Standards of Al-Si-Mg Alloys …............................................. 18

2.1.8 Influence of variability of alloy composition towards fraction solid... 19

2.1.9 Thixotropic raw material enhancements .................................... 20

2.1.10 Measure processability of A356 with Rheocast Quality Index .....21 
2.1.11 Derived strength of A356 from microstructure attributes.............. 22

2.1.12 Conclusion on thixotropic raw material .................................... 22

2.2 Pre-processing of semi-solid metal ................................................ 23

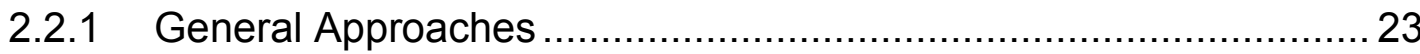

2.2.2 Semi-solid agitation/stirring production of thixotropic raw material . 23

2.2.3 Non-stirring production of thixotropic raw material ........................ 25

2.2.4 Conclusion on pre-process routes for semi-solid metal ................. 28

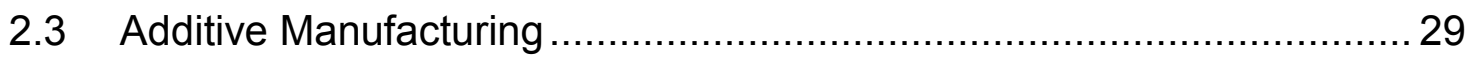

2.3.1 Variations of Additive Manufacturing ........................................... 30

2.3.2 Material Extrusion Additive Manufacturing (MEAM) ....................... 31

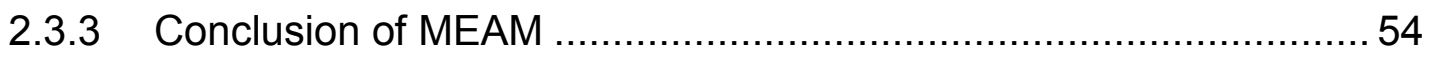

2.4 Research on MEAM with thixotropic/semi-solid metals ....................... 54

2.4.1 Rheocaster approach on MEAM and metal joining ....................... 56

2.4.2 Numeric heat simulation of deposition of aluminum ...................... 58

2.4.3 Solid Freeform Fabrication of metal components by extrusion and

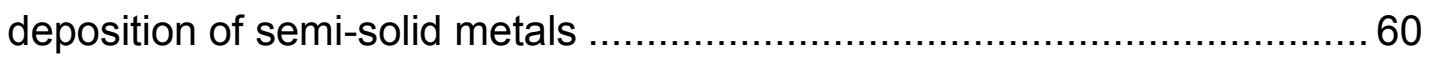

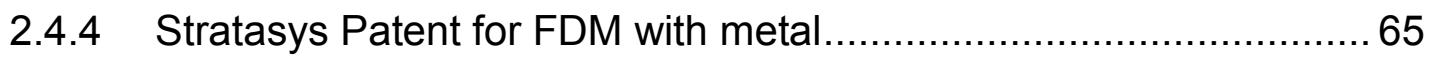

2.4.5 Use of low melting temperature soldering alloys........................... 65

2.4.6 Direct metal writing with rheocasting approach............................. 66

2.4.7 Fast Desktop-Scale Extrusion Additive Manufacturing …............... 68

2.4.8 Induction heating of aluminum alloy into semi solid state .............. 68

2.4.9 Conclusion of MEAM with thixotropic/semi-solid alloys................... 70

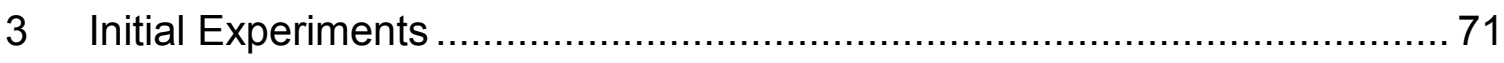

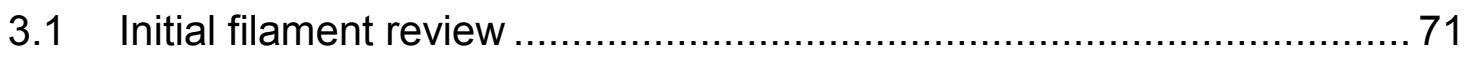

3.2 Metal MEAM test apparatus components and design ........................ 78

3.3 Material choice and filament production ............................................ 82 


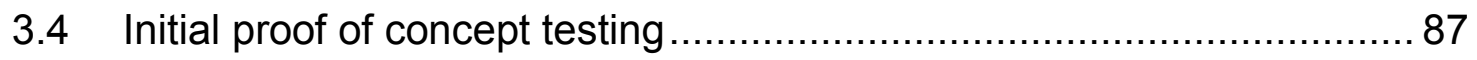

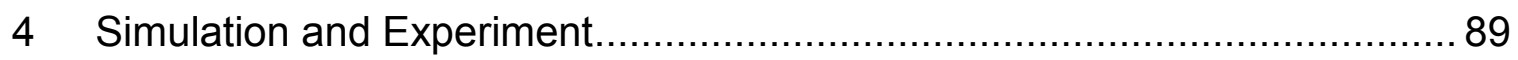

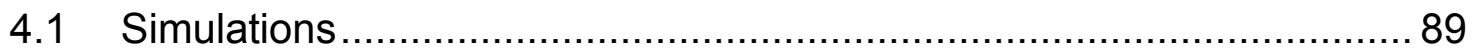

4.1.1 Simulation of heated channel changes ….................................. 89

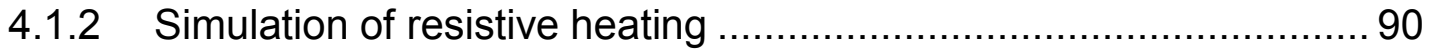

4.1.3 Simulation of inductive heating …........................................... 97

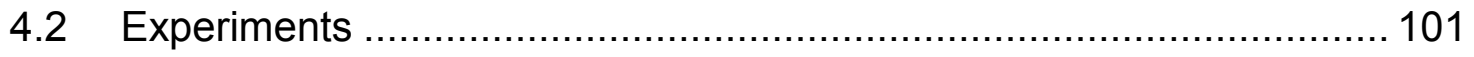

4.2.1 Heating to globular microstructure experiment .......................... 101

4.2.2 Aging experiment ............................................................. 117

4.2.3 Cleaning process development.............................................. 128

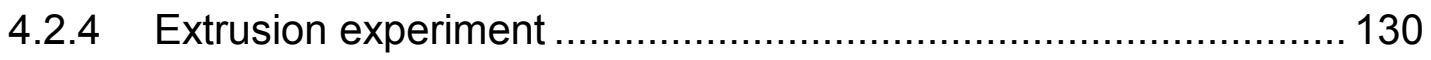

4.2.5 Induction Pre-Heating experiment............................................ 132

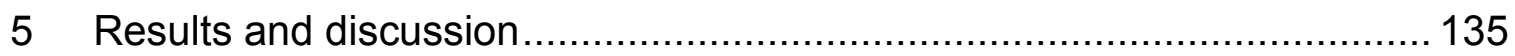

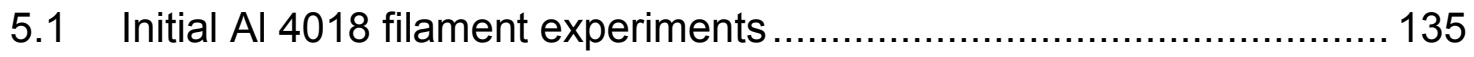

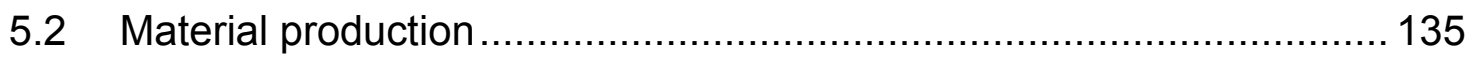

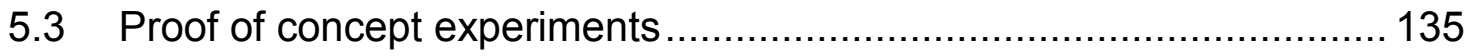

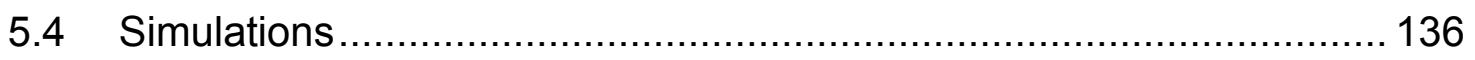

5.4.1 Simulation of heated channel dimension changes ........................ 136

5.4.2 Simulation of resistive heating …........................................... 136

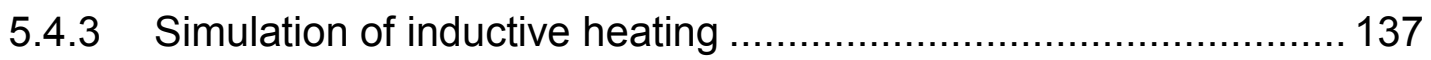

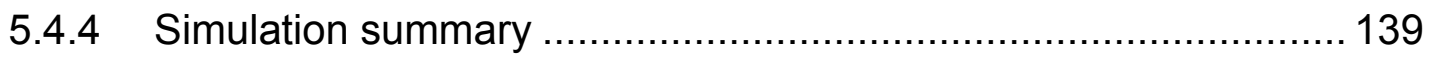

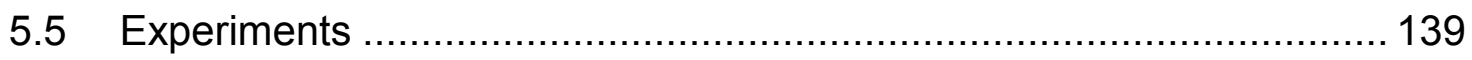

5.5.1 Heating to globular microstructure experiment ........................... 139

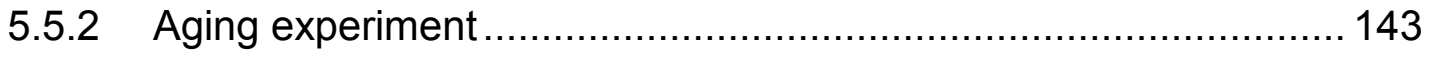

5.5.3 Cleaning process development................................................. 144

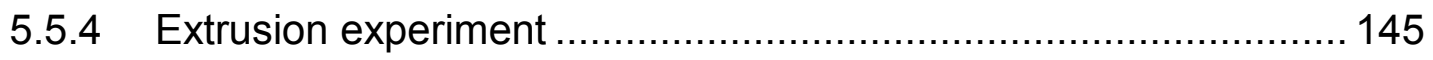


5.5.5 Induction Pre-Heating experiment............................................. 147

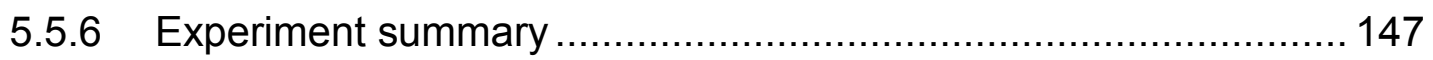

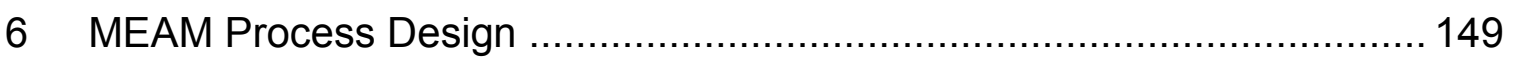

6.1 MEAM feedstock material and shape design ................................ 149

6.2 MEAM Nozzle and machine design ............................................... 152

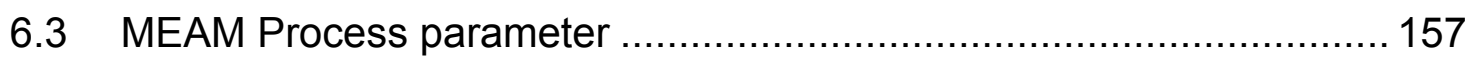

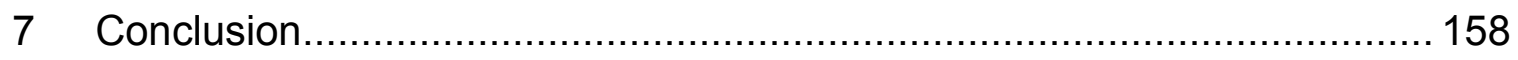

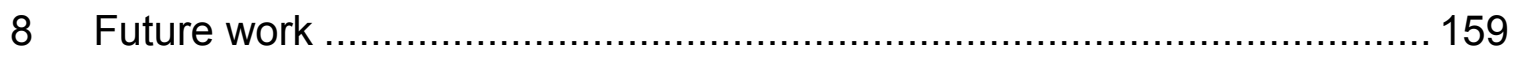

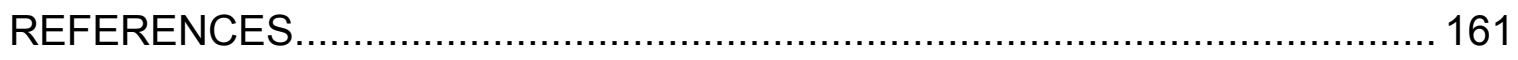

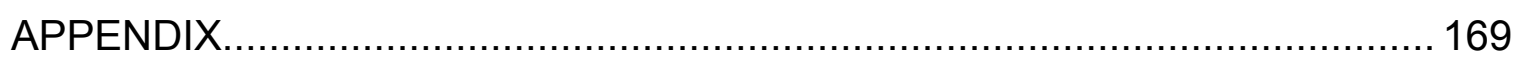

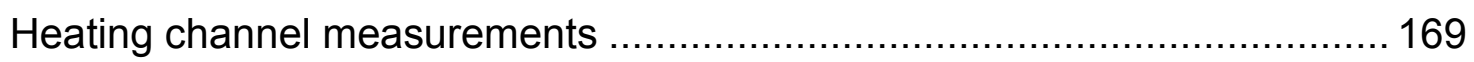

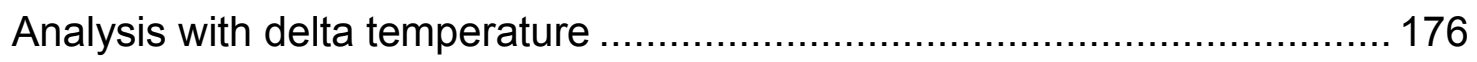

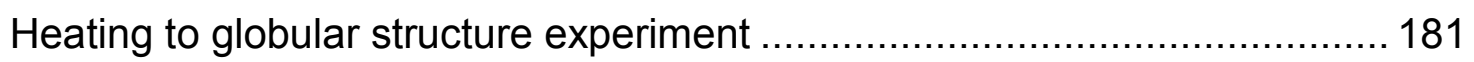

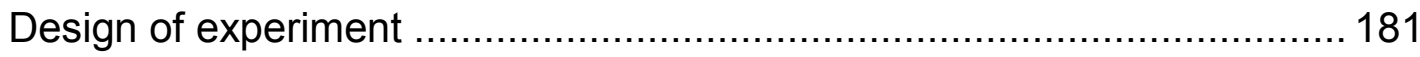

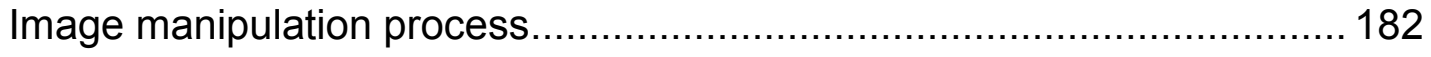

A356 Rheocast Quality Index (RQI) results - Heating Experiment .......... 186

THIXALLOY 540 Rheocast Quality Index (RQI) results - Heating Experiment 189

Count of failures before adequate sample .............................................. 191

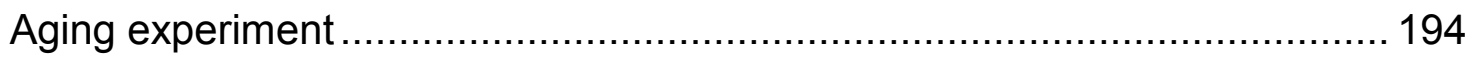

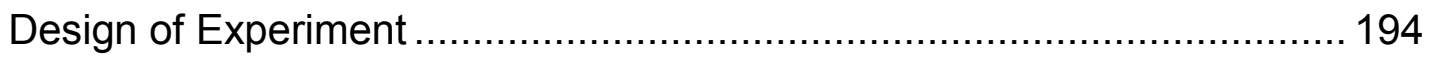

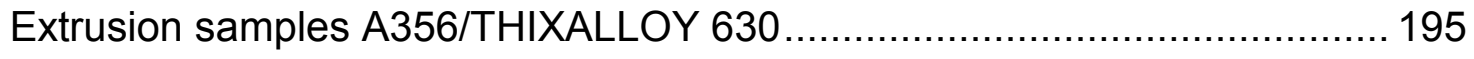

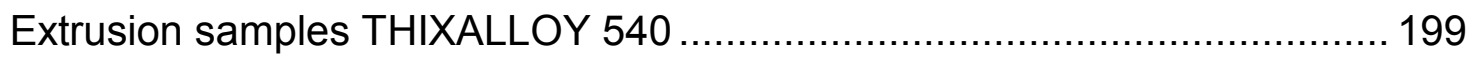

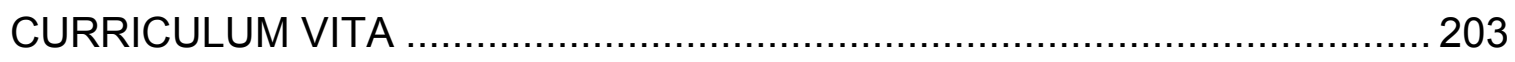




\section{LIST OF FIGURES}

Figure 1 - Face Centered Cubic (FCC) Lattice structure .................................. 4

Figure 2 - Dislocation types: (a) Edge (b) Screw [9] ...................................... 5

Figure 3 - Al-Si Phase Diagram [10] .................................................... 7

Figure 4 - Solidification of an alloy against a cold chill wall (a) columnar (b)

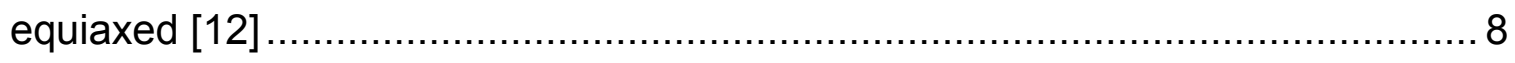

Figure 5 - Aluminum alloy billet at $\mathrm{f}_{\mathrm{s}}=0.5$ exhibits a consistency of modeling clay [13]

Figure 6 - Effect of Cooling Rate on fraction solid [15] ................................. 10 Figure 7 - $(\mathrm{a}, \mathrm{c}, \mathrm{e})$ conventional casting and $(\mathrm{b}, \mathrm{d}, \mathrm{f}) \mathrm{MHD}$ casting after holding at

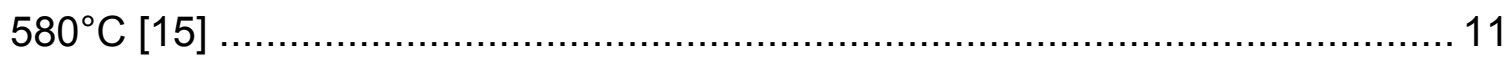

Figure 8 - Schematic Model Flow Curves ................................................... 15

Figure 9 Dependency of slurry viscosity on structural/internal variables ........... 17

Figure 10 - Simplified aluminum alloy system per ANSI H35.1 ........................ 19

Figure 11 - Thermocalc $\circledast$ calculated fraction solid by temperature for AISiMg min

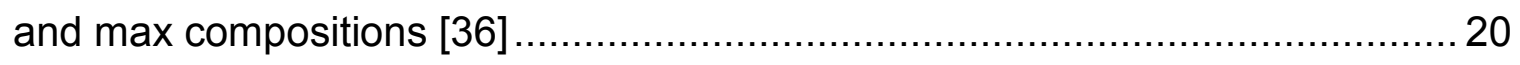

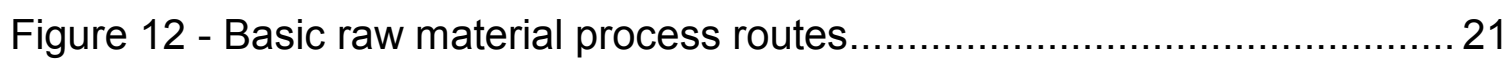

Figure 13 - Stirring modes: (a) mechanical stirring; (b) passive stirring; (c) electromagnetic vertical stirring; (d) electromagnetic horizontal stirring [43] ...... 24 Figure 14 - Grain size of electromagnetic stirred $(\bullet$ ) and cold rolled (०) AlSi7Mg06

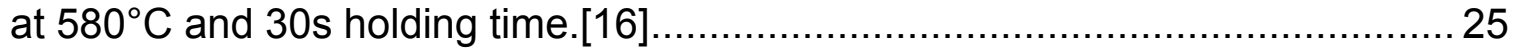

Figure 15 - Time - Temperature Profile of the SIMA Process[45] .................... 26

Figure 16 - Schematic illustration of ECAP processing routes with different or no

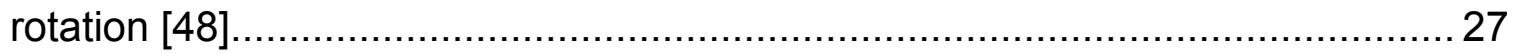

Figure 17 - Suggested continuous ECAS process for filament [51] .................. 28

Figure 18 - Additive Manufacturing process chain [55] ................................. 29

Figure 19 - Metal powder-bed system installations [61]................................. 31

Figure 20 - Schematic of Fused Deposition Modeling ....................................... 32

Figure 21 - fabbster teethed stick feed [64] ................................................. 33 
Figure 22 - Connecting of Fabbster sticks for form filament ............................ 34

Figure 23 - filament pinch roller types 1-steel, 2-aluminum [67] …................... 35

Figure 24 - Filament feed force and shear a) nozzle measurement b) feed rate vs

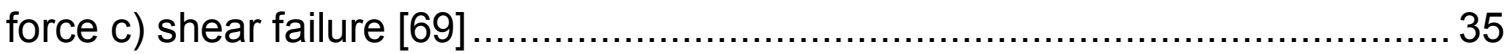

Figure 25 - FastFFF Extruder with nut feeder and laser pre-heater [70]............ 36

Figure 26 - Differential control volume for conduction analysis [73]................. 38

Figure 27 - Temperature drop due to thermal contact resistance [75] ............... 39

Figure 28 - Induction Heating and Skin Depth [76] ...................................... 41

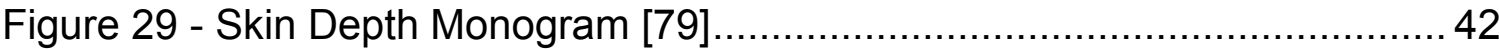

Figure 30 - Inductor-effectiveness per diameter skin depth ration (adapted from

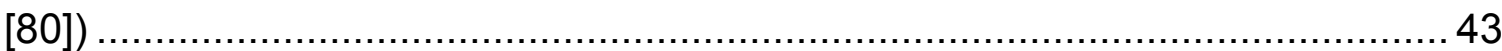

Figure 31 - Extrusion Principle (adapted from [81], [82] and [83]) …............... 44

Figure 32 - Extrusion constant for various metals at different temperatures [85] 45

Figure 33 - Material flow in a planar extrusion die [89] ................................. 47

Figure 34 - Eutectic layer besides the dead zone during A356 extrusion [26] .... 48 Figure 35 - Filament with possible contact areas $A_{1}, A_{2}, A_{4}, A_{5}$ to filament, $A_{3}$ to

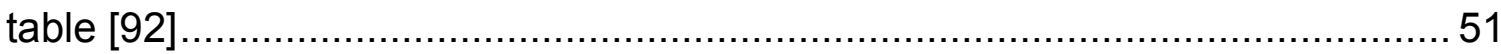

Figure 36 - Simulated time based temperature evolution for 9 filament roads [92]

Figure 37 - Temperature gradient and average approximation of layer interface [71] 53

Figure 38 - time bar showing research results and patents for MEAM with thixotropic or non-eutectic metals at the start of this work ................................ 55

Figure 39 - Rheocaster for extrusion and deposition of semi-solid slurries [3] ...56

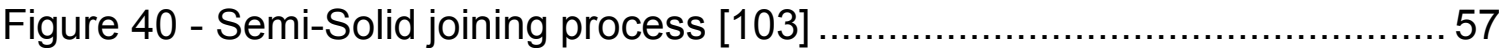

Figure 41 - Sample extruded objects and interface section (middle arrow) [3] ...58

Figure 42 - MEAM setup with rheocaster [104] .......................................... 58

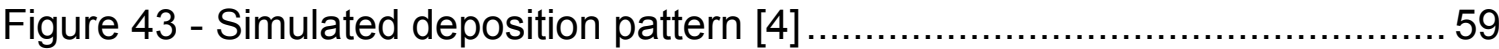

Figure 44 - fully dense deposition height based on the extrusion rate [4] ..........6 60

Figure 45 - Rheological studies of Finke [71] a) capillary viscometer, b) plate-plate rheometer, c) back extrusion experiment 61 
Figure 46 - Force resulting in segregation (solid) vs. extrusion force (dotted) [71]

Figure 47 - Experimental nozzle including force measurement via load cell [71] 63 Figure 48 - Process window for EDSSM a) 3D quantitative representation, b) cross section through the process volume 64 Figure 49 - a) standard curved liquefier of FDM 3000 b) added a strait liquefier [115] 66

Figure 50 - Extrusion examples for a) 2D and b) 6 layer 3D structures [115] ..... 66 Figure 51 - Direct Metal Writing - a) Rheocaster design b) experiment setup [113] 67

Figure 52 - Rheocasting bonding a) adequate bonding with pre-heated substrate b) Inadequate bonding without pre-heating of substrate [113]....................... 67

Figure 53 - FastFFF Extruder with laser pre-heating [70] ................................ 68

Figure 54 - Oxygen shielded feeder for Al-FFF [114] ................................... 69

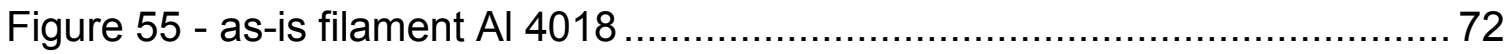

Figure 56 - Time required for the filament to reach $585^{\circ} \mathrm{C}$ in the air-flow oven .. 73 Figure 57 - Picture of micrograph example $585^{\circ} \mathrm{C}$ with automatic grain separation

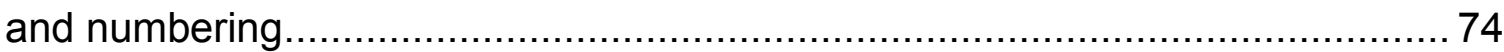

Figure 58 - Al4018 filament micrographs after 15 minutes isothermal holding ... 75 Figure 59 - Graph of average grain diameter and form factor vs. temperature... 76 Figure 60 - Fraction solid vs. temperature ............................................... 76 Figure 61 - Microstructure evolution with the first 5 min of isothermal holding ... 76 Figure 62 - Mass spectrometry of the Elisental filament ................................... 77 Figure 63 - Estimation of the Elisental filament using Thermo-Calc with Al-Si4.29 78

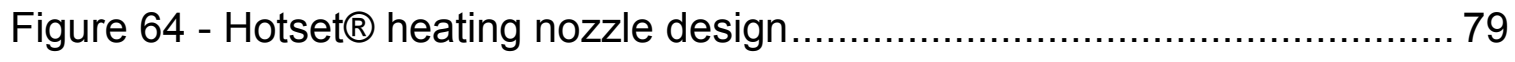

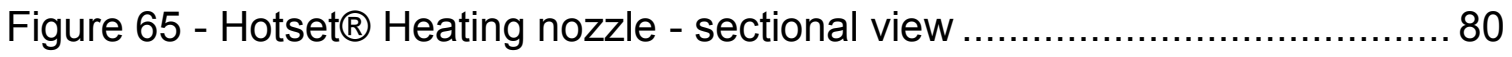

Figure 66 - Resistive heating channel and control unit ................................. 80

Figure 67 - Wire feed and control unit [118] ............................................... 81

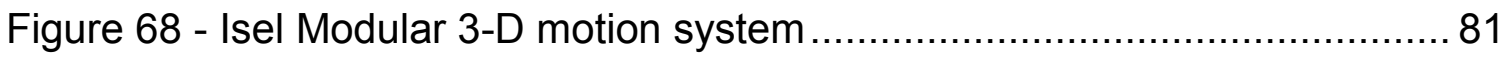

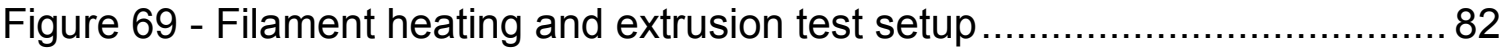

Figure 70 - Raw material $(\mathrm{a}$ - rod, b - reduced bolt) ….................................. 83 
Figure $71-0.5 \mathrm{MN}$ extrusion press and recorded processing parameter..... 84 Figure 72 - A356 (THIXALLOY 630) raw rod material a) 25x magnified b) 50x magnified c) 50x magnified d) 200x magnified 85 Figure 73 - A356 extruded filament a) 50x magnified b) 100x magnified c) 200x magnified d) 500x magnified. 86 Figure 74 - THIXALLOY 540 raw rod material a) 50x magnified b) 100x magnified c) 200x magnified d) 500x magnified... 86 Figure 75 - THIXALLOY 540 extruded filament a) 50x magnified b) 100x magnified c) 200x magnified d) 500x magnified. 87

Figure 76 - Proof of concept extrusion setting 88 Figure 77 - Cracked nozzle insulation due to expansion at elevated temperature 89

Figure 78 - Heating channel simulation a) nozzle entry dimension at ambient temperature b) displacement vector at $585^{\circ} \mathrm{C}$ c)nozzle entry dimension at $585^{\circ} \mathrm{C}$ 90

Figure 79 - A356 Density / Temperature model and approximation .................. 91

Figure 80 - A356 Specific Heat / Temperature model and approximation .......... 91 Figure 81 - A356 Thermal Conductivity / Temperature model and approximation 92

Figure 82 - A356 Viscosity at liquid temperatures model and approximation ..... 92 Figure 83 - Nozzle inside with resistive coil heaters and thermocouple connection 93

Figure 84 - Temperature contour for ideal/uniform nozzle temperature at $585^{\circ} \mathrm{C}$, $2 \mathrm{~mm} / \mathrm{s}$ 93

Figure 85 - A356 Simulation results for ideal/uniform nozzle temperature at $585^{\circ} \mathrm{C}$, $2 \mathrm{~mm} / \mathrm{s}$ Mass fraction liquid and temperature ................................................... 94

Figure 86 - Interval Plot for temperature delta from temperature setting ............ 95 Figure 87 - Fitted line plot and temperature distribution function for $585^{\circ} \mathrm{C} / 858 \mathrm{~K}$

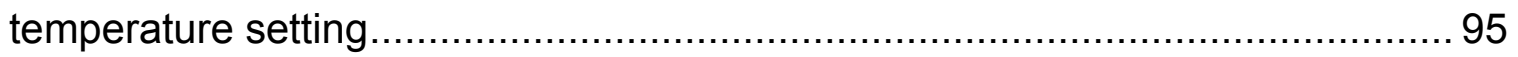
Figure 88 - ANSYS Fluent User defined function to apply the nozzle temperature distribution 96 
Figure 89 - Adapted Simulation Results for A356 heating with nozzle temperature profile Contour for resulting temperature and mass fraction liquid with setting $585^{\circ} \mathrm{C} / 858 \mathrm{~K}$

Figure 90 - A356 Electrical Conductivity / Temperature model and approximation 97

Figure 91 - Ansys induction heating simulation setup with Maxwell and Fluent . 99 Figure 92 - Induction heating simulation geometry design a) 3D model b) 2D

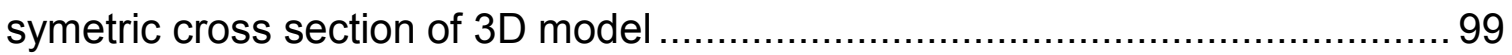
Figure 93 - Ohmic Losses - left) after one simulation pass right) after last simulation pass. 101

Figure 94 - left) Nozzle without tip right) Boron Nitride ceramic spray ............. 102

Figure 95 - Left) Experiment Setup right) sample results.............................. 103

Figure 96 - Power Curve for full factorial design for the heating experiment .... 105 Figure 97 - Example of THIXALLOY 540 low temperature with inadequate microstructure. 106

Figure 98 - Example panorama picture of Microstructure - Sample 18 - A356

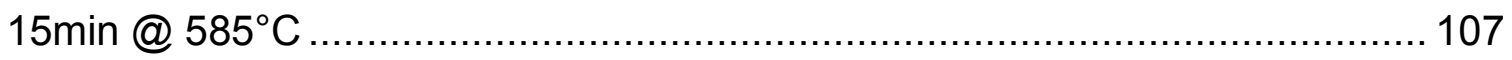
Figure 99 - Example of chosen micrograph for sample 18 middle at 50x maginification 107

Figure 100 - Macro- and microstructure analysis approach by Zoqui et.al. [37] 108 Figure 101 - left) sample micrograph - right) results of macro- and microstructure analysis 108

Figure 102 - Sample 18M image processing a) BW transfer \& smooth b) manual segmentation c) inverted final image for measurement ............................... 109 Figure 103 - Interval plot of RQI for temperature settings, alloy and hold Time 111 Figure 104 - Residuals Plot for the A356 Temperature Model verification........ 112 Figure 105 - Residual Plot for the A356 hold time and temperature effects on RQI 113

Figure 106 - A356 Residual Plots for effects on Grain Size ............................. 114

Figure 107 - THIXALLOY 540 Residuals Plot for RQI ................................... 116

Figure 108 - THIXALLOY 540 Residuals Plot for Grain Size / Diameter .......... 117

Figure 109 - Power Curve for full factorial design for the aging experiment ..... 118 
Figure 110 - Aging example - sample 19: A356 50min @ 585 $\mathrm{C}$ 119

Figure 111 - A356 a) Sample 18M - 15min @ 585 C b) Sample 19M 50min @ $585^{\circ} \mathrm{C}$

Figure 112 - Residual Plot for A356 heating to aging 120

Figure 113 - Main effects plot for RQI - A356 heating to aging..... 121

Figure 114 - Residuals Plot RQI - THIXALLOY 540 Aging ............................ 122

Figure 115 - Main effects plot for RQI - THIXALLOY 540 Aging ..................... 122

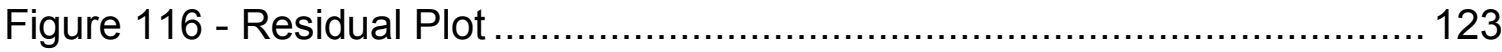

Figure 117 - Main effects plot for grain size - A356 aging …....................... 124

Figure 118 - Residual Plot for Regression Analysis Grain Size - A356 Aging .. 125

Figure 119 - Residuals Plot for Diameter - THIXALLOY 540 Aging ................. 126

Figure 120 - Residual Plot for Regression Diameter - THIXALLOY 540 aging . 127

Figure 121 - Scatterplot with regression Diameter vs Hold Time - THIXALLOY 540 128

Figure 122 - Scatterplot with regression Diameter vs temperature - THIXALLOY 540 128

Figure 123 - Nozzle cleaning left) magnetic mixer right) ultra-sonic 129

Figure 124 - Extrusion experiment piston pins 132

Figure 125 - Small scale induction heating experiment setup 132

Figure 126 - Left) filament sample with drilling right) filament fitted on thermocouple 133

Figure 127 - Initial induction heating temperature results 133

Figure 128 - Interval plot of temperature after 40 seconds 134

Figure 129 - Adapted Simulation Results for A356 heating with nozzle temperature profile diagram for resulting temperature and mass fraction liquid with setting $585^{\circ} \mathrm{C} / 858 \mathrm{~K}$ 137

Figure 130 - Induction heating simulation results - left) Outlet temperature difference Wall -Center for 318A right) Mass Fraction Liquid at the center line 312A 138

Figure 131 - - Induction heating simulation result / sensitivity of the excitation vs. fraction liquid 138 
Figure 132 - A356 main effects of temperature (in ${ }^{\circ} \mathrm{C}$ ) and hold time (in min) on $\mathrm{RQI}$ 140

Figure 133 - A356 contour plot of temperature and hold time (in min) on RQI . 140 Figure 134 - A356 Main effects of hold time (in min) on grain size ................... 141 Figure 135 - THIXALLOY 540 Main effects of hold time (in min) on RQI......... 141 Figure 136 - THIXALLOY 540 Main effects of hold time and temperature on grain size 142

Figure 137 - Holding time prediction model for A356. 144

Figure 138 - A356 extrusion run 5 - left) resulting sample right) microstructure

Figure 139 - THIXALLOY 540 run9 - left) resulting sample right) microstructure 146

Figure 140 - THIXALLOY 540 run 9 extrusion example with ruptured skin ...... 146

Figure 141 - Schematic Bolt design ............................................................ 151

Figure 142 - Schematic MEAM nozzle design ............................................. 153

Figure 143 - Schematic feedstock supply .................................................... 155

Figure 144 - MEAM apparatus - including table and housing ......................... 156 


\section{LIST OF TABLES}

Table 1 - Phase sensitivity and enthalpy of technical used alloys [33] .............. 18

Table 2 Physical Characteristics of A356.2 alloy [35] .................................... 19

Table 3 - AISiMg compositions as per EN 1706:2010 ................................... 19

Table 4 - Advanced Manufacturing variations................................................. 30

Table 5 - Typical conductance with normal surface finish and moderate preasure

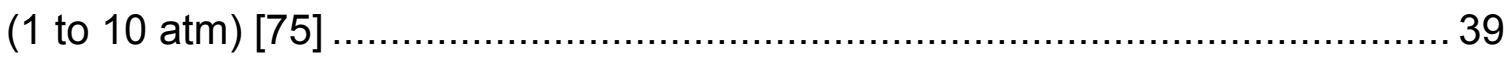

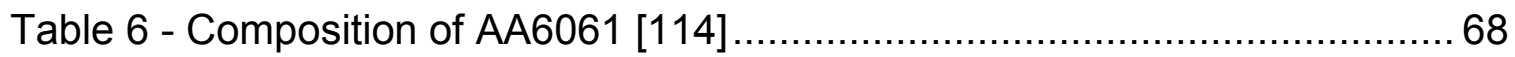

Table 7 - Alloy composition compare Al4018 vs. A356 .................................. 71

Table 8 - filament isothermal holding for $15 \mathrm{~min}$ at different temperatures results

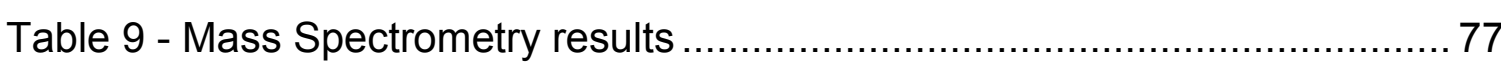

Table 10 - A356 (THIXALLOY 630) / AISi7MG0.5 composition (exclusive Al).... 83

Table 11 - THIXALLOY - 540 / AIMg5Si2 composition (exclusive Al) ................ 83

Table 12 - Filament extrusion process parameter .......................................... 84

Table 13 - Microstructure analysis results of raw and extruded material ............ 84

Table 14 - Frequency calculation based on skin depth and temperature ........... 98

Table 15 - Induction heating simulation run results for excitation of 315 Ampere .. 100

Table 16 - Induction heating simulation run results for excitation of 318 Ampere 100

Table 17- Induction heating simulation run results for excitation of 321 Ampere 100

Table 18 - Initial heating experiment results .............................................. 103

Table 19 - Temperature model for A356 heating samples with marked overlaps . 106

Table 20 - Temperature model for THIXALLOY 540 heating samples ............. 107

Table 21 - Example of individual grain results of sample 18 ........................... 109

Table 22 - Area and perimeter average of Sample 18M ................................ 110 
Table 23 - RQI calculation example for sample 18 …............................... 110

Table 24 - Extrusion experiment design aspects ............................................ 130

Table 25 - Extrusion experiment process ...................................................... 130

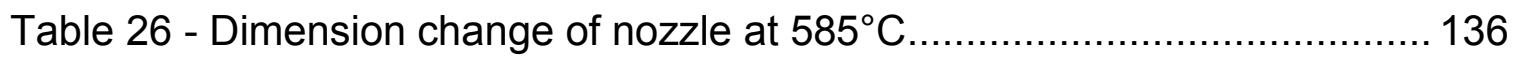

Table 27 - Expected RQI and Grain Size for holding time of 25 min prior extrusion 


\section{INTRODUCTION}

\subsection{Problem Statement}

Several strategies using additive manufacturing (AM) resulting in near net shape metal products have been implemented. Powder bed systems are commercially available to create prototypes, molds and functional parts. Most commercial systems are based on steel, chrome, titanium, nickel or iron materials to produce either direct or "green" parts ${ }^{1}$. Aluminum parts can mainly be produced via the casting route, i.e. creating a wax pattern or sand casting mold via AM and pouring the aluminum part. To allow a higher volume additive manufacturing of aluminum products a direct route to produce parts would be required. Material Extrusion Additive Manufacturing (MEAM) ${ }^{2}$ based Fused Deposition Modeling ${ }^{\circledR}$ (FDM) developed by Stratasys is a well know additive manufacturing process route using a filament to extrude shaped layers of material and therefore layer wise creating a finished product. Current commercialized MEAM filament materials are restricted to thermoplastics such as acrylonitrile butadiene styrene (ABS), polycarbonate (PC) or Polyphenylsulfone (PPSF or PPSU).

Aside of the current use of metal in additive manufacturing, David Spencer [1] and Merton Flemings discovered in 1971 the thixotropic behavior of certain alloys and applied this effect to casting and forging of products. One of the key aluminum alloy that is used in such thixotropic casting routes is $A 356^{3}$. Research on such casting alloys are focused on its use as large ingots in casting and forging environments. Specifics on alloys extruded from filaments as thixotropic slurry

\footnotetext{
${ }^{1}$ Parts that need to be sintered below the melting point to retrieve the required strength or infiltrate with a second material e.g. bronze

${ }^{2}$ Material Extrusion Additive Manufacturing will be used as the generalized term still recognizing that the method was invented by Stratasys Inc. Other terms that have been used for that method are Solid Freeform Fabrication (SFF) and

${ }^{3}$ Aluminum alloy with $\mathrm{Al}, \mathrm{Si}, \mathrm{Zn}, \mathrm{MG}$ as per ANSI H35.1
} 
have only been completed using low melting $\mathrm{Pb}$ and $\mathrm{Sn}$ and extrapolating for aluminum alloys by Finke et al.[2], Rice et al.[3] and Vijh et al.[4]. Another route for direct printing of aluminum is the use of single molten aluminum droplets as proposed by Cao \& Miyamoto [5] or Qi et al. [6]. The aluminum alloy MEAM route has been progressed in 2000/2001 but since then just numerically been simulated by Vijh et al. in 2009 [4].

\subsection{Research Contributions}

This dissertation defines the possible routes for using as-is or pre-processed aluminum alloy filament for layer-based extrusion in a semi solid state. It compares the existing paths of pre-production of cast alloys and illuminates the most promising routes. Simulations and experiments of driving, heating and extruding of such aluminum alloy filaments are performed. Individual process parameters such as extrusion temperature, velocity, holding time and designs are validated via a prototype thixotropic extrusion nozzle.

This dissertation is structured in nine main chapters. After the introduction (this chapter) chapter 2 will review the literature for aluminum alloy raw material, the pre-processing of aluminum alloys for semi-solid use, the key aspects of MEAM as the chosen additive manufacturing process and the latest results on additive manufacturing with semi-solid metals. Chapter 3 will provide details of an initial filament experiment and the MEAM apparatus design that is proposed to be used during this research. Chapter 4 will describe the performed simulations and experiments as well as show the outcome. Chapter 5 will take the outcomes of the prior chapter and derive decisions for the following simulations and experiments as well as derive models for application in a MEAM apparatus. Chapter 6 will take all the discussed results and derive a machine and process design. Chapter 7 will conclude and summarize the work while chapter 8 will define the proposed next steps in a research towards an operational MEAM machine. 


\section{LITERATURE REVIEW}

This dissertation will combine an existing research and application area of using semi solid aluminum in casting and forging with Material Extrusion Additive Manufacturing (MEAM) as a specific additive manufacturing process. The literature review will therefore go through published research areas starting with thixotropic raw materials and review it from more fundamental aspects towards the latest developments. As a next area additive manufacturing with its specific application of MEAM will be reviewed. As referred to in section "1.1 Problem Statement" there is limited research on using thixotropic / semi-solid metals applied to MEAM. This area will be reviewed last and the research topic and contribution will be discussed as well as a work plan derived from that.

\subsection{Thixotropic raw material}

\subsubsection{Fundamentals of aluminum alloys}

To understand the thixotropic behavior of an aluminum filament one would need to review the basics of metals, metallic alloys and their phase diagrams [7]. The first concept to recognize is that of crystals. Metal crystals have a specific structure defined by its lattice parameter i.e. the distance between single atoms and their angle within a certain unit. A unit that is repeated within the overall structure is called a "unit cell." Unit cells can be simple ${ }^{4}$ or stacked with additional atoms. The most important crystal structures are face centered cubic (FCC), body centered cubic (BCC) and hexagonal closed packed (HCP). Pure Aluminum exhibits an FCC structure (see Figure 1) which means the cube of 4 aluminum atoms houses 6 more atoms - a single one on each outward facing side.

\footnotetext{
${ }^{4}$ Simple unit cells can be cubic, tetragonal or hexagonal
} 


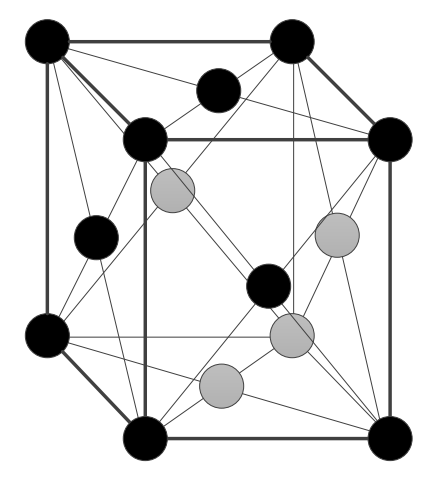

Figure 1 - Face Centered Cubic (FCC) Lattice structure

Another key concept of metals are the irregularities of a lattice structure. Those will later become very important in creation of a thixotropic raw material.

The first model for irregularities is a point defect. The point failure can be a vacancy, which means that a place which under normal conditions is being occupied is vacant. The other less frequent defect is the self-interstitial which is an atom trapped in a place in which under normal conditions an atom would not be included. The point defects may also include impurities. Alloys are the result of intentionally adding one or more types of atoms together, which typically become solutes ${ }^{5}$ or a solvent ${ }^{6}$. Such a solute atom can, depending on its size, become a substitutional or interstitial atom. Substitutional atoms replace a solvent atom whereas interstitials atom will assemble into a space between the solvent atoms. If a solute atom will differ in size more than $\pm 15 \%$ from the solute, it will create a significant deviation from the pure solvent lattice structure. Such a solid solution will result in a new phase as it exhibits significant changes to its attributes. During review of semi-solid slurries the types of phases become a fundamental aspect to understand phase diagrams. As part of understanding a phase the concept of concentration is vital as in semi-solid slurries phases with different compositions may exist. The composition or concentration for one component out of a binary metal alloy is defined in Equation 2.1 as:

${ }^{5}$ Element in minor concentration

${ }^{6}$ Element in greatest concentration - also known as host atoms 


$$
C_{1}=\frac{m_{1}}{m_{1}+m_{2}} \times 100
$$

Such a composition can be calculated as weight percent or atomic percent.

The further important types of irregularities are dislocations and interfacial defects resulting in grains. Dislocations can be separated into edge also known as line dislocations and screw dislocation (see Figure 2). For semi-solid slurries such dislocations are used as seeds for grain growth especially during the Strain Induced Melt Activated (SIMA) process to create raw material for the thixotropic route. Edge dislocations are a terminated line of atoms within a plane as per Figure 2a.

The screw dislocation is a region of atoms that is shifted by one atomic distance (see Figure $2 b$ ). Both the screw and edge dislocations generally appear as hybrid dislocations. Any dislocation significantly increases the energy of the lattice structure. The dislocation density in annealed metal is about $10^{6} \mathrm{~mm} / \mathrm{mm}^{3}$ (resulting in a dislocation line length of $1 \mathrm{~km}$ per $\mathrm{mm}^{3}$ ) and can be raise through cold processing to about $10^{10} \mathrm{~mm} / \mathrm{mm}^{3}(10000 \mathrm{~km})$. For each $1 \mathrm{~mm}$ of dislocation line, the energy increases by about $10^{-12}$ Joules. This results in an assumed maximum increase of energy of $10^{-2} \mathrm{~J} / \mathrm{mm}^{3}$. [8]

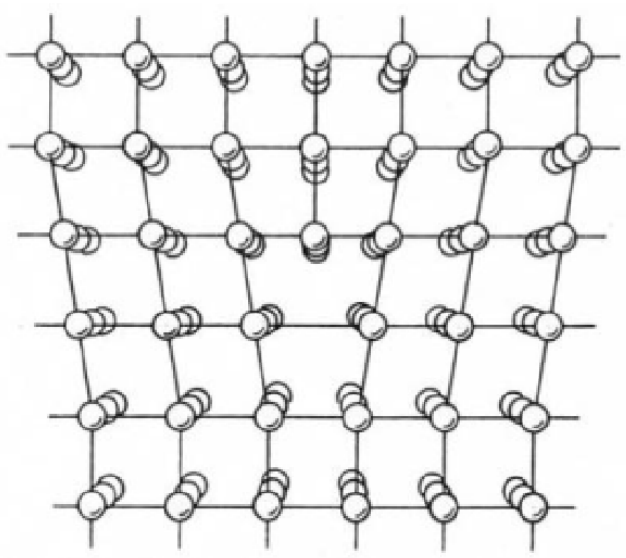

(a)

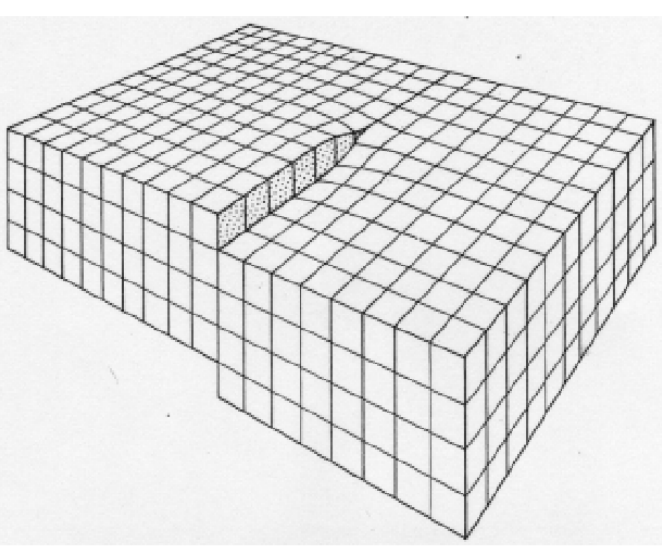

(b)

Figure 2 - Dislocation types: (a) Edge (b) Screw [9] 
Another important defect is the interfacial one. It includes external surfaces, defects in crystals, and grain and phase boundaries. During cooling of a metal in its liquid state below the liquidus temperature, solidification typically starts at walls, interfaces or dislocations with the appearance of a nucleus ${ }^{7}$. To grow further a nucleus requires a critical radius otherwise it will re-dissolve. Each nucleus will grow if cooled further until it reaches a wall or another nucleus. As the direction of the crystal is random in normal cases the boundary of the finished growing nuclei will show different crystallographic orientations. Individual crystals with a single orientation are called grains and the touching lines / areas are called grain boundaries. In solid alloys the additional boundaries that can be observed are phase boundaries which separate the phases with different chemical composition ${ }^{8}$.

To understand the cooling behavior of an alloy it is important to understand its phase diagram. An important concept besides the shown temperature, liquid semisolid, solid phase and concentration is the solubility of a compound within a second one. In the case of A356 the key solutes are silicon ( $\mathrm{Si}$ ) and magnesium (Mg). Depending on the temperature there is a maximum concentration of the solute e.g. Si that can dissolve in the solvent e.g. Al.

A binary aluminum silicon alloy is a eutectic alloy and does have a full solubility in the liquid phase as well as a limited solubility at room temperature. For the assumed thixotropic alloy the overall composition of $\mathrm{Si}$ will be chosen above the maximum solubility (see Figure 3 ) at room temperature (e.g. $1.65 \% \mathrm{Si}$ ) and the below the composition at the eutectic point (e.g. 12.6\% Si). When cooled from the pure liquid state and past the liquidus line a primary phase will form. This will continue until the eutectic temperature $\mathrm{T}_{\mathrm{e}}$.

\footnotetext{
${ }^{7}$ Known as heterogeneous nucleation

${ }^{8}$ Note that phases are a portion with uniform physical and chemical properties as such a different state (liquid, solid, gaseous) is also seen as a different phase e.g. for a pure material.
} 


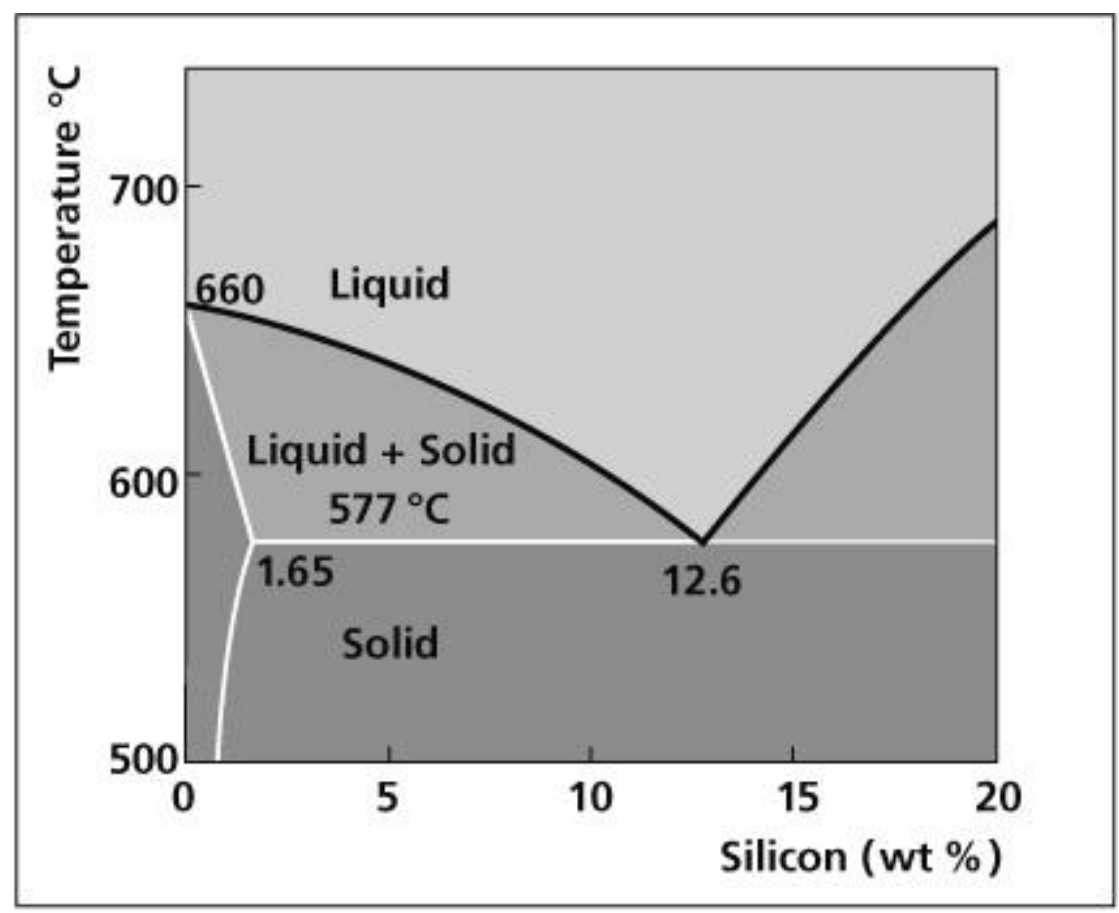

Figure 3 - Al-Si Phase Diagram [10]

If the temperature falls below the eutectic temperature the eutectic phase will crystallize to include eutectic $\alpha$ and $\beta$ phases that exhibit a repeating lamellar structure. A eutectic alloy composition solidifies directly from a pure liquid to the full solid phase at the eutectic temperature.

\subsubsection{Fundamentals of thixotropic alloy behavior}

Thixotropic behavior was discovered by Schalek and Szegvari in 1923 through experiments with iron oxide gels [11]. They found that the gel becomes completely liquid through gently shaking but solidifies after a period of time. This concept of time dependent viscosity when applying shear stress was called thixotropy. It was reviewed that microstructural breakdown of 3D structure and its re-establishment is the cause of the time dependent thixotropic shear thinning. In 1971 David Spencer with his Professor Merton J. Flemings discovered that metal alloys can be processed while in such a time dependent semi-solid state [12] (see Figure 5) as long as dendritic breakdown and therefore creation of spherical grains within the solid phase happens. The basic principle behind the behavior lies in the dendritic solidification of metals (see Figure 4 ) which is observed in nearly all metal 
alloys. If then stirred during a semi solid state these dendritic structures break and form spherical solid particles, enabling flow with less shear stress than before.

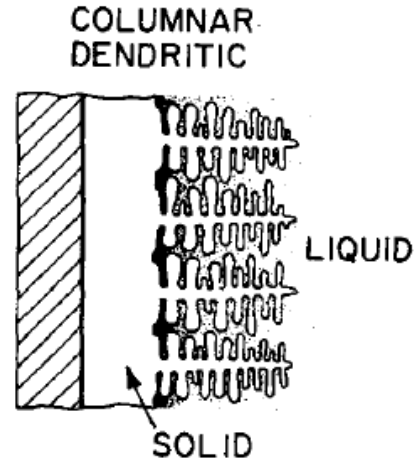

(a)

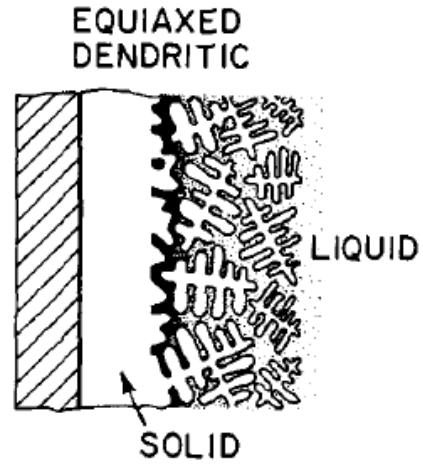

(b)

Figure 4 - Solidification of an alloy against a cold chill wall (a) columnar (b) equiaxed [12]

\subsubsection{Semi-solid states of thixotropic alloys}

During solidification the fraction liquid/ solid is a key attribute to determine whether it can be processed or not. The fraction liquid can be modeled by the Scheil equation using the composition of the liquid at a given location $\left(C_{L}\right)$, composition of the initial alloy $\left(\mathrm{C}_{0}\right)$ and a constant partition ratio $(\mathrm{k})$ as shown in equation 2.2:

$$
f_{L}=\left(\frac{C_{L}}{C_{0}}\right)^{-\frac{1}{(1-k)}}
$$

As the liquid composition $\left(C_{L}\right)$ is related to temperature, Scheil's equation can be written using a dimensionless temperature $\phi=\left(T_{M}-T\right) /\left(T_{M}-T_{L}\right)$ where $T$ is the temperature of the alloy, $T_{M}$ is the melting point of the pure solvent (e.g. Al) and $T_{L}$ is the liquidus temperature of the alloy:

$$
f_{L}=\phi^{-\frac{1}{(1-k)}}
$$

Both composition and temperature based equations measure the fraction of solid/liquid based on weight. Assuming that the liquid and solid has the same density the fraction would also reflect the volume fraction of solid. 


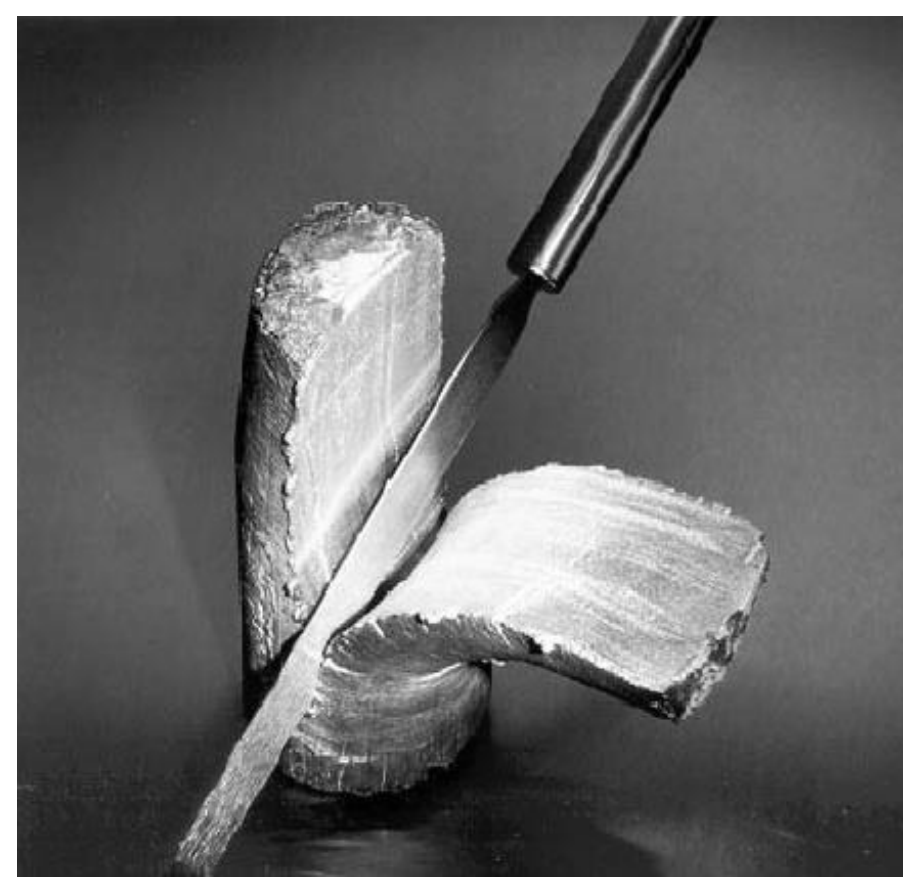

Figure 5 - Aluminum alloy billet at $f_{s}=0.5$ exhibits a consistency of modeling clay [13]

Scheil's equations[14] neglect two processes that exist in semi-solid metals and results in a somewhat lower fraction liquid than that calculated from the equation[12]. First one is diffusion or micro segregation in the solid phase (assumption for Scheil's equation $D_{s} \rightarrow 0^{9}$ ). For the liquid phase a complete diffusion / homogenous distribution is expected $(D\llcorner\rightarrow \infty)$ for Scheil's equation. As long as the cooling rates are sufficiently high, e.g. $\gg>1 \mathrm{Ks}^{-1}$ (see Figure 6 ) the effect can be neglected as a typical diffusion coefficient of $D=10^{-13} \mathrm{~m}^{2} \mathrm{~s}^{-1}$ requires several hours to occur at a center of a $100 \mu \mathrm{m}$ diameter spherical particle[15].

${ }^{9} D_{s}$ defines the mass transfer from liquid into the solid phase 


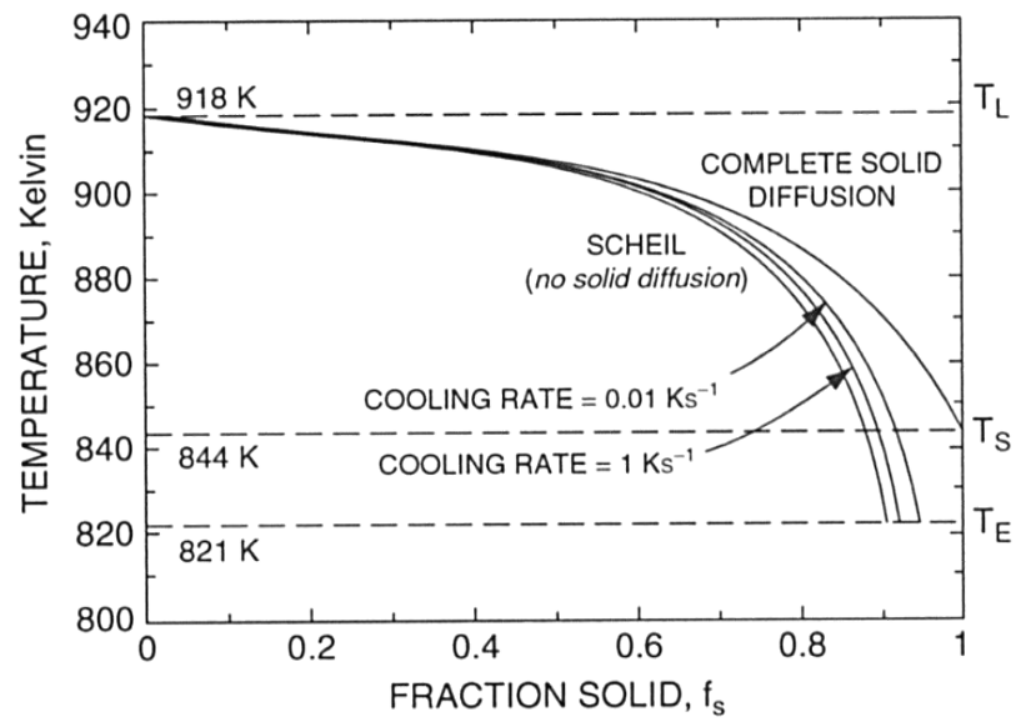

Figure 6 - Effect of Cooling Rate on fraction solid [15]

The second aspect neglected by Scheil's equation is coarsening via Ostwald Ripening and coalescence. It results in fusing of single grains into a larger one or the filling of spaced between dendrite arms and therefore the increase of the grain size in order to reduce the area between the solid - liquid interface.

\subsubsection{Shape and structure of thixotropic alloy particles}

Ostwald Ripening and coalescence is time dependent and can be described by the following equation 2.4 [16]:

$$
S_{v}=S_{v 0}(1+k t)^{-\frac{1}{3}}
$$

$\mathrm{S}_{\mathrm{v}}$ represents the solid - liquid interface surface area per unit volume, $t$ the time and $S_{\mathrm{v} o}$ is the initial value before isothermal holding. The rate constant $k$ in $\mathrm{min}^{-1}$ was determined for AISi7Mg0.6 between 0.3 and $1.8 \mathrm{~min}^{-1}$ (electromagnetically stirred and non-stirred). Figure 7 [15] shows the initial and isothermal hold structure of electromagnetically stirred and conventional AlSi7Mg0.6 alloy at $580^{\circ} \mathrm{C}$.

Additional measurable or derived shape and structure attributes of thixotropic alloys are grain size of the solid phase, number of grains per unit area, average globule diameter, volume fraction of the solid phase and a resulting shape factor. 

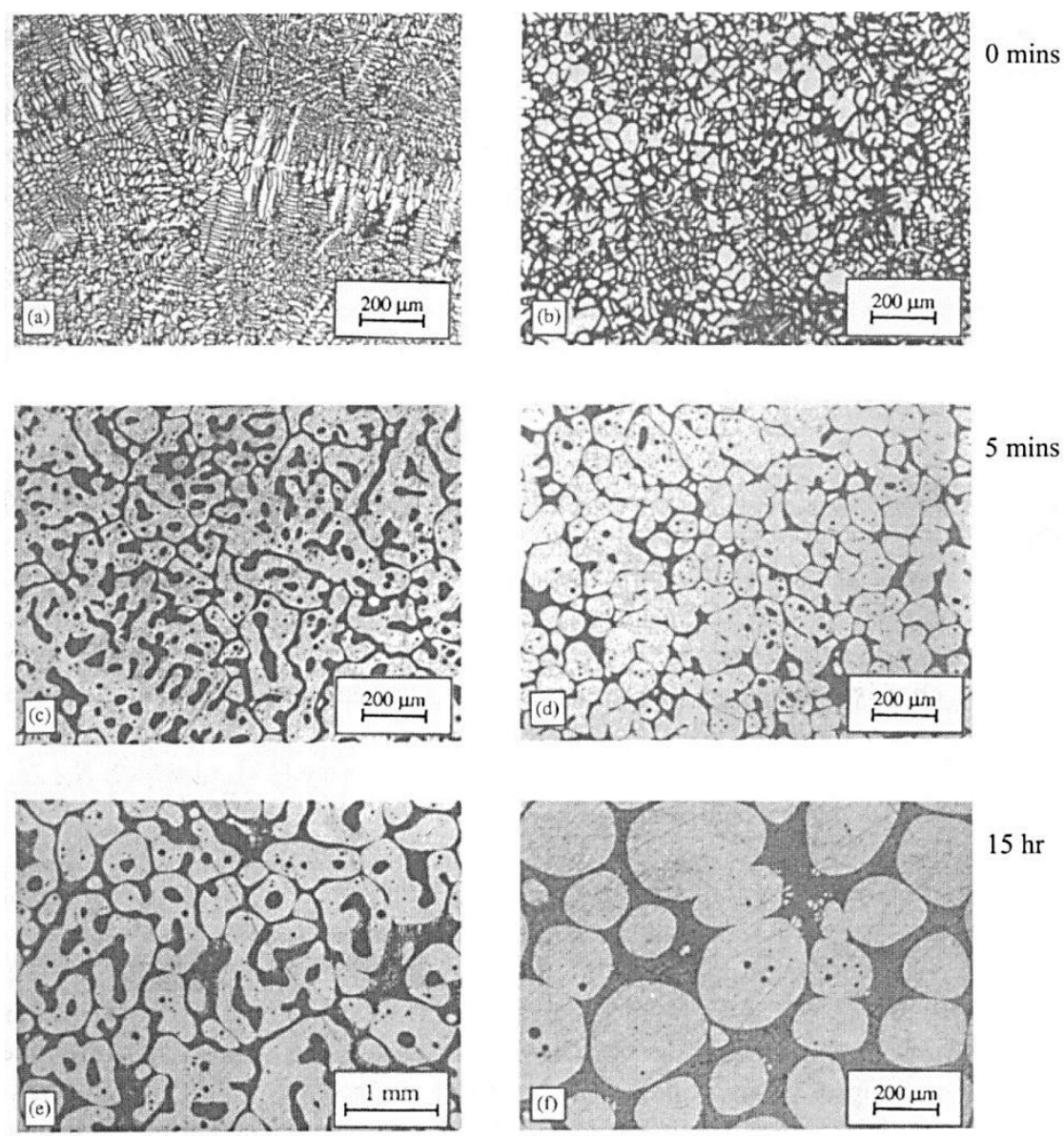

Figure $7-(a, c, e)$ conventional casting and $(b, d, f)$ MHD casting after holding at $580^{\circ} \mathrm{C}[15]$

The number of grains per unit and their final size is dependent on the development of initial nucleus or the cut off of dendrite arms within the agitated semi-solid slurry. It has to be noted that nucleus or broken off particles require either enough free energy i.e. through undercooling or need to have reached a critical size. Otherwise the nucleus or particle will melt/dissolve [8]. A common standard to determine the grain size is ASTM e112 for manual or ASTM e1382 for semi or full automatic determination [17]. The most manual method proposed by ASTM e112 is the comparison of a magnified acid treated and polished specimen with a standard chart. Aluminum is proposed to be compared to plate I at a magnification of $100 x$.

The volume fraction of solid at a given temperature can be determined by quenching of a semi-solid specimen to capture the microstructural phase status. Quenching is the process of extreme chilling that result in the creation of single phase metal with the fraction liquid in a 'frozen' state [8]. Based on that state the 
fraction solid at the quenched temperature is visible as single phase a grains under a microscope. Tzimas and Zavaliangos [18] reviewed the volume fraction approximation methods ${ }^{10}$ and found no superior ones - each with unique advantages.

The above defined solid liquid interface surface area per unit volume $S_{v}$ can be determined for a specimen using image analyzing techniques by measuring the total solid liquid interface length $\left(L_{\alpha}\right)$ and the chosen area of the specimen $(A)$ :

$$
S_{v}=\frac{4}{\pi} \frac{L_{\alpha \mid}}{A}
$$

The fraction of the solid phase $\left(f_{\alpha}\right)$ can be determined by the total solid phase area $\left(A_{\alpha}\right)$ and the chosen area of the specimen $(A)$.

$$
f_{\alpha}=\frac{A_{\alpha}}{A}
$$

Using the number of grains $\left(\mathrm{N}_{A}\right)$ in the specimen area a grain specific shape factor $\left(F_{g}\right)$ defined by Loué and Suéry [16] can be determined:

$$
F_{g}=\frac{1}{6 \pi f_{\alpha}} \frac{S_{v}{ }^{2}}{N_{A}}
$$

This shape factor shows value larger than 1 for complex e.g. dendritic grain structures and equals 1 for perfectly spherical solid phase grains. A more simple form factor used for semi solid metal processing is defined as:

$$
f=\frac{4 \pi A}{U^{2}}
$$

with $A$ being the grain area and $U$ the length of the grain contour.

Further, an important attribute of the structure is called contiguity (C) and was introduced by Gurland [19]. It defines the average fraction of the surface area of a

10 Used approximation methods: equilibrium phase diagrams, thermal analysis techniques, quantitative metallography on microstructures of quenched 
solid particle that is shared with its neighbor of the same phase. The contiguity is defined as:

$$
C=\frac{2 S_{\alpha \alpha}}{2 S_{\alpha \alpha}+S_{\alpha \beta}}
$$

$S_{\alpha \alpha}$ represents the area of interface between the $\alpha$ particles and $S_{\alpha \beta}$ between $\alpha$ and $\beta$ particles (or eutectic phase). $C$ will equal 0 for no contact and 1 for full contact i.e. in a fully solid state.

The volume contiguity calculated by multiplying the contiguity with the fraction solid:

$$
V_{C}=f_{S} C
$$

This attribute is one of the reasons for the solid behavior of slugs with a liquid fraction above 0.4 .

\subsubsection{Viscosity, Shear and plastic behavior of semi-solid aluminum alloys} Viscosity $(\eta)$ is the measure of resistance of a fluid to being deformed. The rate of deformation is called strain $(\gamma)$, its velocity strain rate $(\dot{\gamma})$ and the force divided by the area cross sectional is called shear stress $(\tau)$. The basic relationship between viscosity, strain rate and shear stress is called dynamic viscosity:

$$
\tau=\eta * \dot{\gamma}
$$

When viscosity is constant over time and for different shear stresses the fluid is called Newtonian. If the viscosity decreases with shear stress it is called shear thinning whereas if its increases it is called shear thickening. The general power law type viscosity model (known as the Ostwald and de Waele model) for all behavior to shear stress introduces a consistency factor $(k)$ and a power law index (n) [20]:

$$
\tau=k \dot{\gamma}^{n} \quad \text { or } \quad \eta=k \dot{\gamma}^{n-1}
$$

The power law index defines the type of behavior, with $n=1$ for Newtonian, $n>1$ for shear thickening and $\mathrm{n}<1$ for shear thinning. 
If change in viscosity is dependent on time it is called thixotropic for (decreasing viscosity) or rheopectic (increasing viscosity). An additional behavior is the requirement of a yield stress $\left(\tau_{0}\right)$ to start deformation. Such fluids are called Bingham fluids and can again behave from stress as a Newtonian (ideal Bingham Plastic), shear thinning or shear thickening fluid (see Figure 8). The complete power law model including Bingham behavior can be written as [21]:

$$
\eta=\frac{\tau_{0}}{\dot{\gamma}}+k \dot{\gamma}^{n-1} \quad \text { or } \quad \tau=\tau_{0}+k \dot{\gamma}^{n}
$$

Aluminum alloys can behave in several ways. Thixotropic behavior relates to processing of aluminum and other alloys. This behavior is mainly caused by breaking the dendritic structure in a semi solid state. If the alloy is allowed to recreate the dendritic structure over time it returns to a higher viscosity. However this is not the only behavior a semi-solid alloy exhibits. If the alloy is agitated or otherwise forced to create globular particle structures in the semi-solid state it can behave rheopectically by further reducing the viscosity over time especially through Ostwald Ripening when no dendritic growth is applicable anymore. When applying rapid stress changes the semi-solid alloy exhibits shear thickening which has no time dependence[22]. 


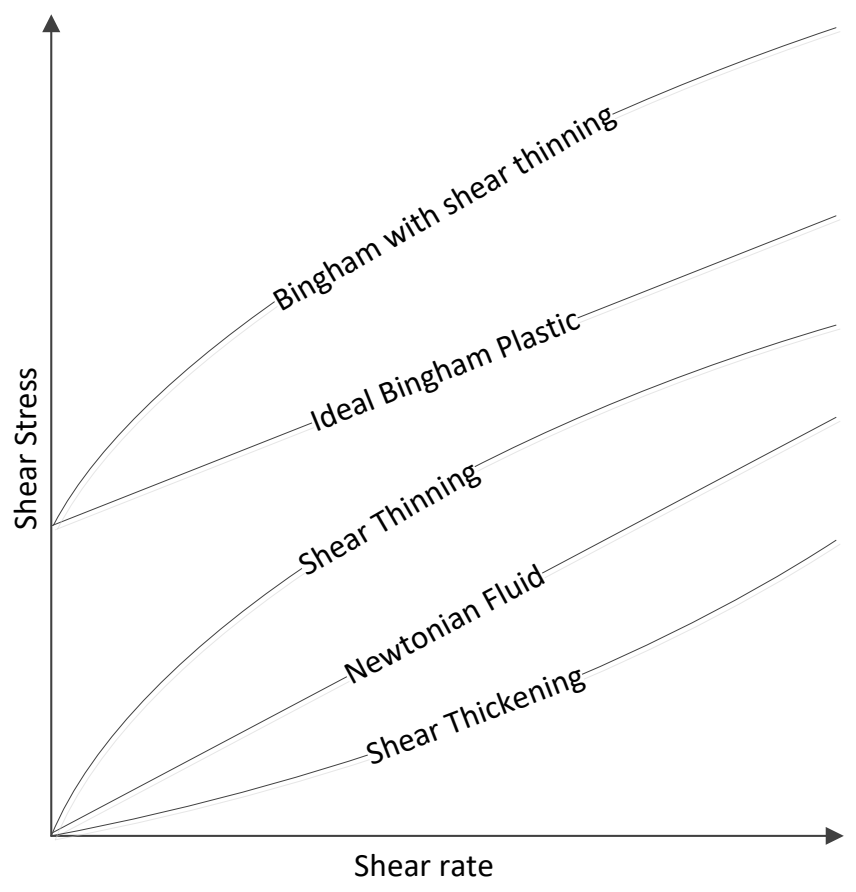

Figure 8 - Schematic Model Flow Curves

For semi-solid metals in general a yield shear stress can be observed [23] and as such have a Bingham like behavior. But compared to an ideal Bingham fluid semisolid metals do not employ a linear relationship between shear stress and shear rate above the yield stress. A simple approximation of yield stress for Al-Si alloys with a solid fraction below 0.6 has been developed by G.K. Sigworth [24]:

$$
\tau_{0}=9615 * \frac{\mathrm{f}^{3}}{\mathrm{f}_{m}-\mathrm{f}}(\mathrm{Pa})
$$

The maximum packing fraction of spherical solid grains $f_{m}$ equals 0.6. Pan et.al [23] reviewed this approximation and found that for A356 the model predicts the yield stress reasonably well up to a fraction solid of about 0.5 .

Another model that has been implemented in the commercially available simulation tool ProCast [25] is called the Power Law Cut-Off (PLCO) model that assumes the semi-solid slurry to be a viscous, isotropic and incompressible fluid. This model therefore assumes (as the models shown before) the material being a one phase 
material. The model was developed and verified by Orgeas et al. and is described as a modified power law relationship by [26]:

$$
\begin{array}{ll}
\mu=\mu_{0}\left(\frac{\dot{\gamma}}{\dot{\gamma}_{c}}\right)^{n-1} & \text { if } \dot{\gamma} \geq \dot{\gamma}_{0} \\
\mu=\mu_{0}\left(\frac{\dot{\gamma}_{0}}{\dot{\gamma}_{c}}\right)^{n-1} & \text { if } \dot{\gamma}<\dot{\gamma}_{0}
\end{array}
$$

The model assumes that for a shear thinning behavior the shear rate $\dot{\gamma}$ needs to be higher than a cut-off shear rate $\dot{\gamma}_{0}$. Below that value the viscosity will be constant. In this model $\mu_{0}$ is defined as a temperature dependent viscosity at a specific shear rate $\dot{\gamma}_{c}$ (Orgeas et al. used $1 \mathrm{~s}^{-1}[26]$ ) and can be calculated via:

$$
\mu_{0}=\mu_{\text {liquid }} \exp \left(B f_{s}\right)
$$

The specific viscosity $\left(\mu_{0}\right)$ is therefore based on the temperature which is represented by the fraction solid $\left(f_{s}\right)$, the parameter $\mathrm{B}$ that is mainly dependent on the microstructure of the semi-solid metal and the viscosity $\left(\mu_{\text {liquid }}\right)$ of the pure liquid phase which behaves as a Newtonian liquid.

The shear rate sensitivity coefficient $(n)$ is also dependent on the fraction solid $f_{s}$ and can be calculated as follows:

$$
n=\frac{1-n_{\min }}{2}\left[1-\tanh \left(\frac{2 \alpha\left(f_{s}-f_{s, 0}\right)}{1-n_{\min }}\right)\right]+n_{\min }
$$

As defined by Orgeas et al. the model for $n$ results in the asymptotic limits for low fraction solid ( $n=1$ - Newtonian behavior) and a high fraction solid $\left(n_{\min }\right)$ with a 'slope' $\alpha$ at a critical fraction solid $f_{s, 0}$.

The PLCO model has been validated with an axisymmetric Poiseuille-type extrusion experiment and besides its simplicity with using only 6 rheological parameters it can model the full range of temperature / fraction solid.

To describe the flow behavior e.g. viscosity without external variables and knowing the history Kumar [22] proposed models using internal or structural variables (see Figure 9). 


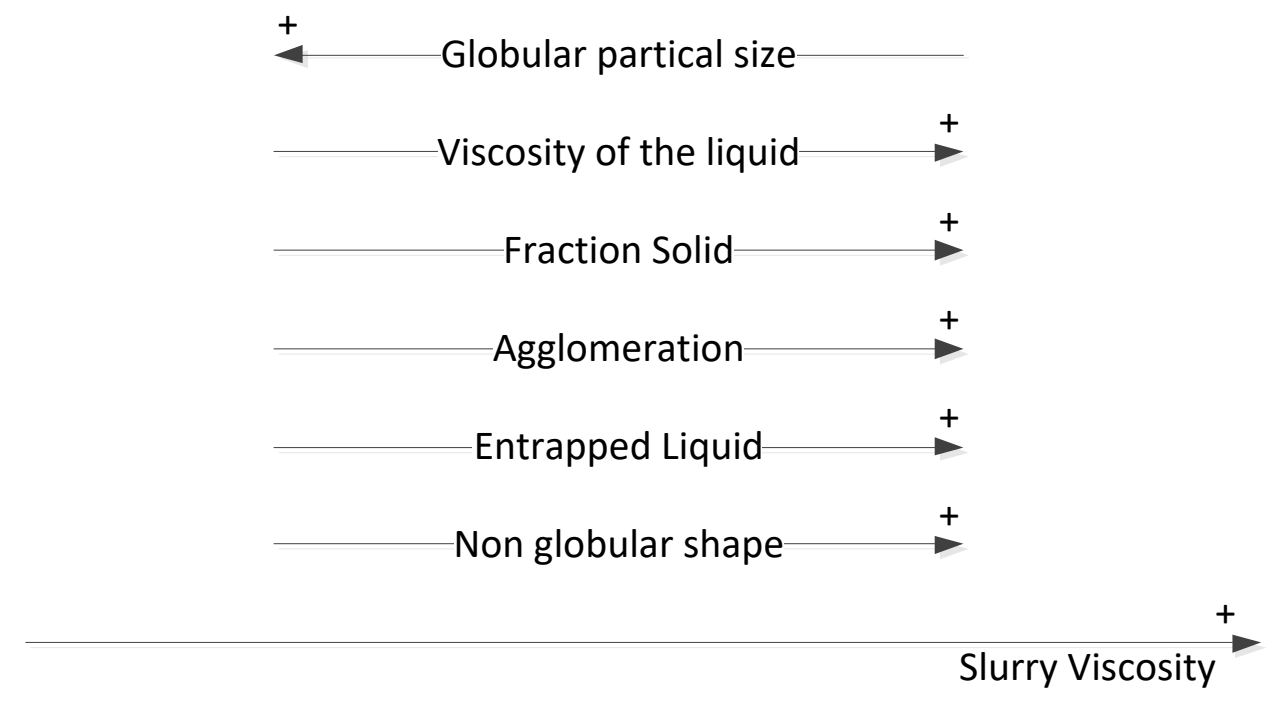

Figure 9 Dependency of slurry viscosity on structural/internal variables

The models above do assume the semi-solid slurry as a one phase material. Several attempts [27], [28], [29], [30], [31] have been made to derive two phase models that treat the liquid and the solid phase differently. The solid phase is treated as a solid skeleton modeled as a pure viscous and compressive medium. The liquid phase is treated as a Newtonian liquid and each phase has its own behavior that will influence the other behavior [15]. The calculation of such models would include a simultaneous processing of a solid fraction, pressure, $2 x$ velocity for solid and liquid and a temperature field. The resulting computation requirements and the need for a larger set of boundary values have prevented those types of models from appearing in industry simulation software packages as of now. It is also assumed that the modeling of a simple MEAM case may not need a two phase model but could rely on a one phase model. A good overview of one and two phase models proposed, compared and used for simulations can be found in the paper of H.V. Atkinson [32].

\subsubsection{Temperature and energetic behavior of thixotropic alloys}

During thixotropic processing the key attribute of the slurry for an adequate viscosity is the fraction solid. The fraction solid is dependent on the precise and homogenous temperature adjustment throughout the slurry within the machine. A 
key influence of the processability of an alloy is expressed by the change of the enthalpy for a $1^{\circ} \mathrm{K}$ change in temperature:

$$
L^{*}=\left|\frac{d H}{d T}\right|
$$

The second important attribute is the temperature difference between the solidus and liquidus line. This can be expressed by the alloy specific temperature sensitivity $\left(S^{*}\right)$ of the fraction solid $\left(f_{s}\right)[33]$.

$$
S^{*}=\left|\frac{d f_{s}}{d T}\right|
$$

For both attributes (see Table 1) the AISi7Mg, AISi5Mg, AZ91 and AM60 are suitable due to small temperature sensitive of the solid phase fraction but on the other side the sensitive alloys AISI1Mg and AE42 are not temperature sensitive towards the added or removed energy.

Table 1 - Phase sensitivity ${ }^{11}$ and enthalpy of technical used alloys [33]

\begin{tabular}{|l|c|c|c|c|c|c|}
\hline & AISi7Mg & AISi5Mg & AlSi1Mg & AZ91 & AM60 & AE42 \\
\hline $\mathbf{S}^{*}\left[\% \mathrm{~K}^{-1}\right]$ & 0.83 & 0.91 & 4.0 & 0.87 & 1.33 & 2.5 \\
\hline $\mathbf{L}^{*}\left[\mathrm{Jcm}^{-3} \mathbf{K}^{-1}\right]$ & 11.5 & 11.8 & 47 & 5.9 & 8.9 & 16.5 \\
\hline
\end{tabular}

AlSi7Mg is the basis for the A356 standard and widely researched in it behavior. It will be used as the basic material for this dissertation also due to its availability.

\subsubsection{Standards of Al-Si-Mg Alloys}

Standards for aluminum alloys (see Figure 10 for a simplified example) have been adopted in 1957 by the American National Standards Institute (ANSI) as ANSI H35.1[34]. The Unified Numbering System (UNS) ${ }^{12}$ runs in parallel to the ANSI system e.g. A356.0 is represented as A13560.

\footnotetext{
${ }^{11}$ Average values based on $0.4<\mathrm{f}_{\mathrm{s}}<0.6$ (exception AISi7Mg $0.5<\mathrm{f}_{\mathrm{s}}<0.6$ )

12 managed by ASTM International (ASTM) and Society of Automotive Engineers (SAE)
} 


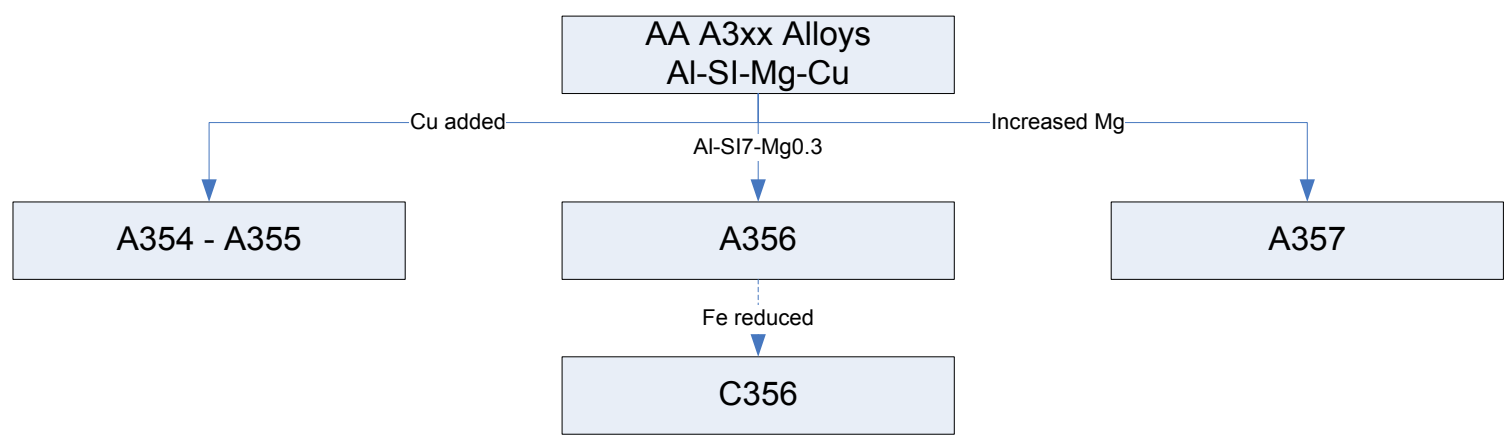

Figure 10 - Simplified aluminum alloy system per ANSI H35.1

Any standard alloy with increasing post digit number has a reduced maximum ratio for non-key components but therefore still falls into the limits of the group e.g. A356 - key components Al-Si-Mg with reduced Fe percentage 0.2 for A356.0 down to 0.12 for A356.2.

Table 2 Physical Characteristics of A356.2 alloy [35]

\begin{tabular}{|l|l|}
\hline Attribute & Values \\
\hline Density & $2.686 \mathrm{~g} / \mathrm{cm}^{3}$ \\
\hline Freezing Range & Liquidus $615^{\circ} \mathrm{C}\left(1140^{\circ} \mathrm{F}\right)-$ Solidus $555^{\circ} \mathrm{C}\left(1035^{\circ} \mathrm{F}\right)$ \\
\hline Coefficient of Thermal Expansion & $23.5 \mu \mathrm{m} / \mathrm{mK}\left(13.1 \mu \mathrm{in} / \mathrm{in}^{\circ} \mathrm{F}\right)$ at $20-300^{\circ} \mathrm{C}\left(67-572^{\circ} \mathrm{F}\right)$ \\
\hline Thermal Conductivity & $\mathrm{T} 51 \mathrm{Sand} 167 \mathrm{~W} / \mathrm{mK}\left(96 \mathrm{Btu} / \mathrm{ft}{ }^{*} \mathrm{hr}{ }^{* \circ} \mathrm{F}\right)$ \\
\hline Latent Heat of Fusion & $389 \mathrm{~kJ} / \mathrm{kg}(167 \mathrm{Btu} / \mathrm{lb})$ \\
\hline Electrical Conductivity & $\mathrm{T} 51$ Sand $43 \% \mathrm{IACS}\left(40.1 \mathrm{n} \Omega^{*} \mathrm{~m}\right)$ at $20^{\circ} \mathrm{C}$ \\
\hline
\end{tabular}

\subsubsection{Influence of variability of alloy composition towards fraction solid} Standard alloy compositions such as EN AC-42100 (A356.0 as per AA) are defined within alloy composition constraints. Basic definitions are done through a main component e.g. Al that is the main proportion defined by $100 \%$ - all additional components, key added alloy components defined by max and min proportion and additional components for refinement or impurities defined by max proportion.

Table 3 - AISiMg compositions as per EN 1706:2010

\begin{tabular}{|l|c|c|c|c|c|c|c|c|c|c|}
\hline Name & Chemical & Al & $\mathbf{S i}$ & $\mathbf{M g}$ & $\mathbf{F e}$ & $\mathbf{C u}$ & $\mathbf{M n}$ & $\mathbf{Z n}$ & Ti & Others \\
\hline EN AC-42100 & AlSi7Mg0.3 & balance & $0.65-0.75$ & $0.3-0.45$ & $\max 0.15$ & $\max 0.03$ & $\max 0.1$ & $\max 0.07$ & $\max 0.18$ & $0.03-0.1$ \\
\hline EN AC-42200 & AlSi7Mg0.6 & balance & $0.65-0.75$ & $0.5-0.7$ & $\max 0.15$ & $\max 0.03$ & $\max 0.1$ & $\max 0.07$ & $\max 0.18$ & $0.03-0.1$ \\
\hline
\end{tabular}


The variability especially in $\mathrm{Si}$ and $\mathrm{Mg}$ has an effect on the attributes of the alloy. This has been studied by Kaufmann et.al [36] for processability especially through fluidity and fraction solid.

Kaufmann et.al showed significant differences in the alloy behavior e.g. phase temperature sensitivity $\left(S^{*}\right)$ at $50 \%$ fraction solid could change from an average value (see Table 1) about $0.8 \%$ per Kelvin (e.g. at AISiMg min) to a very high sensitivity for AISi7Mg max (see Figure 11) at 50\%.

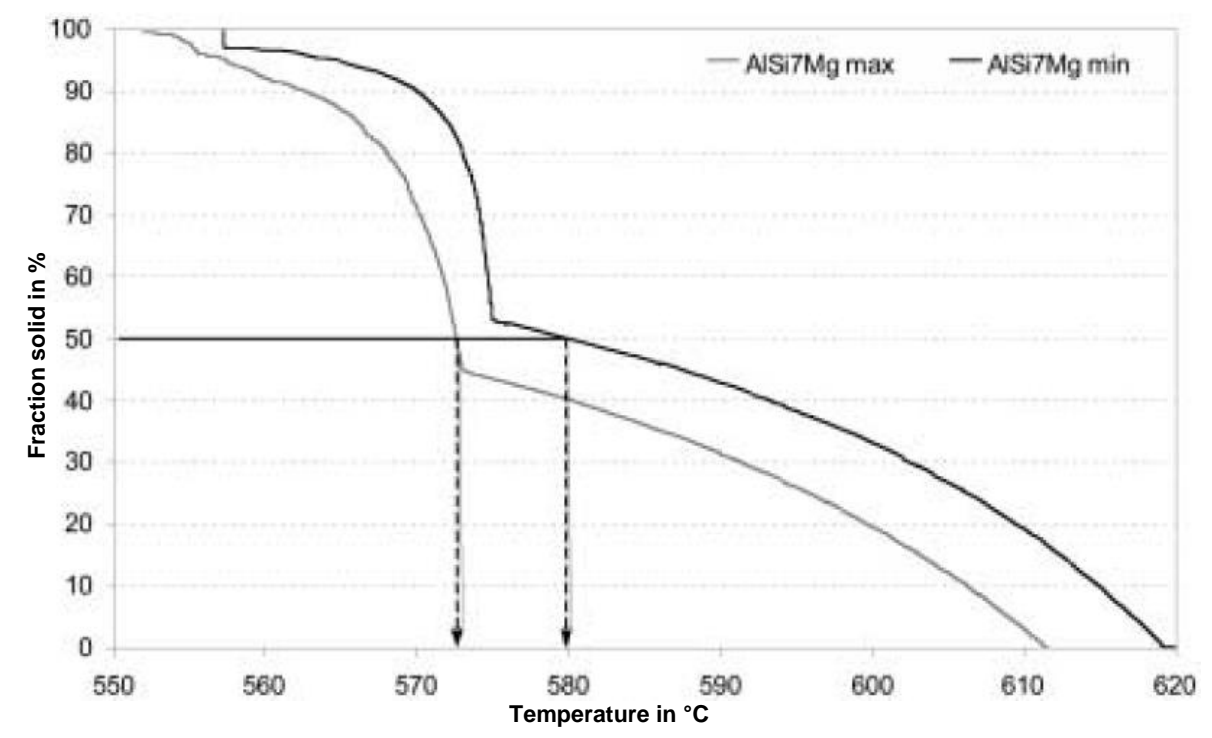

Figure 11 - Thermocalc ${ }^{\circledR}$ calculated fraction solid by temperature for AISiMg min and max compositions [36]

\subsubsection{Thixotropic raw material enhancements}

Several process routes have been identified to achieve a thixotropic / time dependent shear thinning behavior. Initially Spencer [1] physically stirred Sn-Pb alloy from a liquid state to a semi solid state. During further research different paths were discovered to create material that can be processed to achieve a thixotropic state with an adequate behavior. Process routes include (see Figure 12) chemical composition changes, cold working to increase dislocation density/energy, stirring and pure temperature processing. 


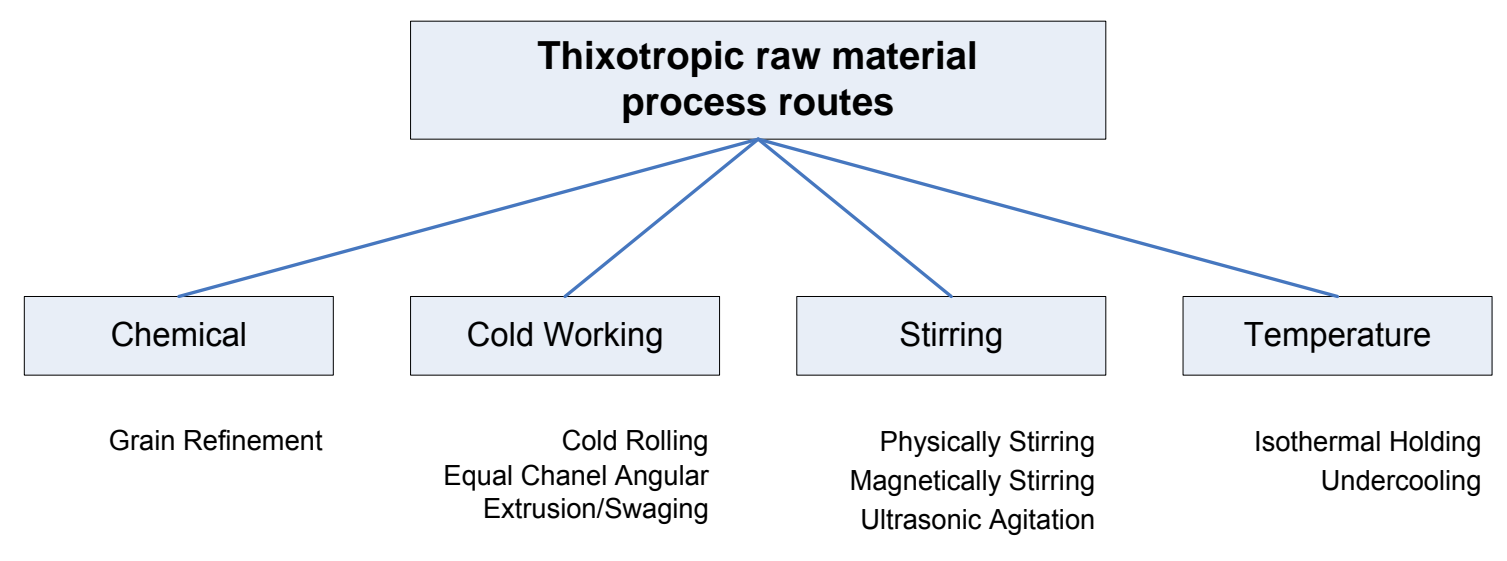

Figure 12 - Basic raw material process routes

Process techniques resulting from the basic routes will be further discussed in Section 2.2 .

\subsubsection{Measure processability of A356 with Rheocast Quality Index}

To measure the applicability of semi-solid alloys for processing within thixoforming or Rheocasting a quality index was developed called the Rheocast Quality Index (RQI) as defined by Zoqui [37]. The index is defined as:

$$
R Q I=\frac{\text { globule size }}{\text { grain size } x \text { shape factor }}
$$

The shape factor used is different than the one used by Loué and Suéry [16] (see Equation 2.7) and is defined by the area and perimeter of the primary phase:

$$
\text { shape factor }=\frac{P_{\alpha}^{2}}{4 \pi A_{\alpha}}
$$

Zoqui et.al derived the apparent viscosity based on the shearing rate and the determined $R Q$ as follows:

$$
\mu(\text { Pas })=\left(2.37+53.6544 e^{(-5.62 * R Q I)}\right) \dot{\gamma}^{(-0.5)} * 10^{5}
$$

The proposed equation can be used to simulate the apparent viscosity when knowing the shearing rate and the RQI. 


\subsubsection{Derived strength of A356 from microstructure attributes}

When either casting or producing using additive manufacturing it is critical to understand the resulting mechanical properties of the final or interim product. To standardize the representation of these quality attributes for A356 a quality index for casting has been designed by Drouzy et.al in 1980 [38]. This index condensed mechanical properties as follows:

$$
Q=U T S+K * \log (\text { Elongation })
$$

with UTS being the ultimate tensile strength. $K$ is a material constant e.g. equivalent to $150 \mathrm{Mpa}$ for $\mathrm{A} 356$. As an alternative to testing of the mechanical properties Khomamizadeh and Ghasemi [39] proposed the microstructural attributes dendrite arm spacing (DAS), porosity and spherodicity to predict the mechanical attributes via the quality index. The result of the mechanical experiments and microstructural analysis of different A356 samples enabled Khomamizadeh \& Ghasemi to approximate the quality index using linear regression as follows:

$$
\begin{gathered}
\operatorname{Qi}(M p a)=213-0.081 * D A S(\mu m)+126 * \text { spheroicity }-24 \\
* \text { porosity }(\%)
\end{gathered}
$$

The differences in microstructural attributes arise at different positions in a cast mold and through different solidification times.

The quality index proposed by Drouzy, Jacob et al. was reviewed as valid especially for A3xx alloys but may not be valid for A2xx alloys. Alexopoulos [40] reviewed other quality indices for validity for different aluminum cast alloys. A review of other quality indices becomes interesting when one would significantly deviate from the standard A356 e.g. by adding grain refinement or changing composition for reducing the oxygen affinity (corrosion).

\subsubsection{Conclusion on thixotropic raw material}

Semi-solid/thixotropic aluminum alloys have a good potential as a filament for material extrusion based additive manufacturing. A basic assumption of this thesis 
is that the composition and morphology of the filament will be a critical factor for the successful use of thixotropic slurries during the material extrusion. A356 or A367 are the most commonly used aluminum alloys for processing in a semi-solid state. Due to their mechanical attributes and wide process window (temperature) they have good potential for use in MEAM. It becomes clear that not just the composition but also the history and resulting morphology of a potential A356 filament is important e.g. resulting grain size, grain shape and entrapped liquid in the semi-solid state. As such the pre-processing for a potential filament must be reviewed to conclude a route and define initial experiments.

\subsection{Pre-processing of semi-solid metal}

\subsubsection{General Approaches}

The process to achieve a semi solid feedstock can be separated into two basic routes Rheocasting \& Thixoforming [15]. The Rheocasting route starts from a fully liquid alloy which is agitated during solidification to produce a slurry which will be directly injected into the mold. The Thixocasting/Thixoforming route includes a complete solidification of steered or otherwise prepared (e.g. SIMA) slurry and later re-heating into partially re-melted slurry. The rheocasting route using liquid metal to create a slurry is not applicable to a filament based Material Extrusion process. As such only the thixoforming route will be discussed further. Nevertheless some Rheocasting processes can be used to prepare a filament or pre-filament rod.

\subsubsection{Semi-solid agitation/stirring production of thixotropic raw material}

Mechanical stirring was the first process route used for creation of semi-solid slurries. A new type of mechanical stirring was developed by the MIT and is called Semi-Solid Rheocasting $\left(\mathrm{SSR}^{\circledR}\right)$. Rheocast processes include an agitation/stirring and rapid cooling process component. $\mathrm{SSR}^{\circledR}$ combines the two process components by inserting a cold/cooled spinning rod into the alloy just above the liquidus temperature. The liquid metal at the rod will be rapidly cooled and pushed outwards into the liquid metal which results in a fine dendritic structure [41]. Within 
the flowing liquid the dendrite arms will coarsen and those arms that are too small will melt off (see 2.1.4 for minimum nucleus/particle size).

A similar method is the gas induced semi-solid (GISS) process [42] by which also a liquid alloy just above the liquidus temperature is agitated through the injections of a graphite diffusor that applies an inert gas into the liquid alloy. Similar to the $\mathrm{SSR}^{\circledR}$ process the injected gas will rapidly cool and agitate the liquid and new solid particles. The SSR ${ }^{\circledR}$ and GISS process claim to offer semi-solid slurries with a low fraction solid $0.1-0.2$ that provide improvement during pouring without modification of the die casting machine.

A completely different feedstock production method still relying on particle agitation is call Magneto-Hydro-Dynamic (MHD) casting or extrusion (see Figure 13). This method is the most commercialized method of producing thixotropic feedstock [22]. The advantage of this method is that it can be added to a commercial caster or extruder so that the melt is agitated at the correct process step.

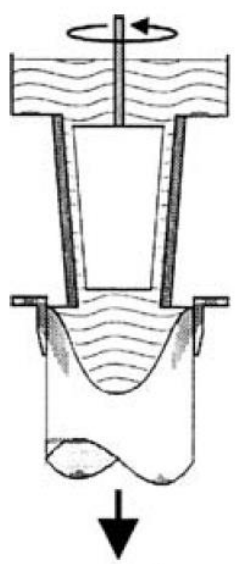

(a)

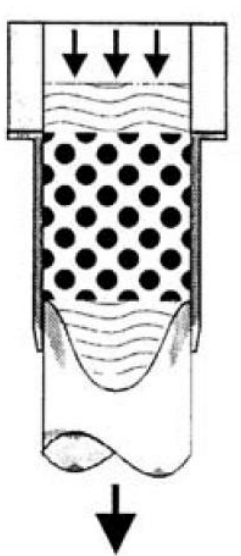

(b)

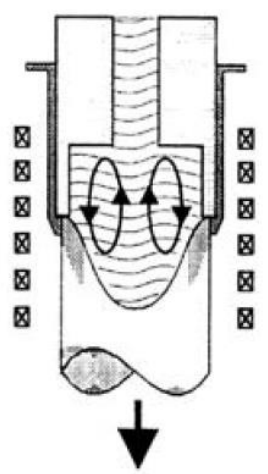

(c)

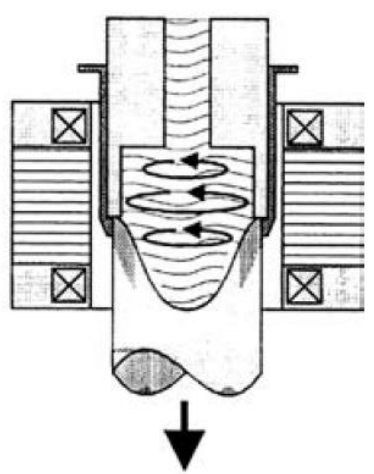

(d)

Figure 13 - Stirring modes: (a) mechanical stirring; (b) passive stirring; (c) electromagnetic vertical stirring; (d) electromagnetic horizontal stirring [43]

Electromagnetic stirring can be applied in a vertical, horizontal or hybrid mode. It results in agitation of the liquid phase. This moving liquid will disturb the growing dendrites in the cooling zone and when those break they will typically form rosettes. Several casting devices with electromagnetic stirring components are patented mainly as mentioned before as an addition to continuous casters e.g. [44]. 


\subsubsection{Non-stirring production of thixotropic raw material}

The Strain Induced Melt Activated (SIMA) process was developed by Young et.al and patented in 1983 [45]. This process does not include a particle agitation during a fluid or semi-solid state. It utilizes the changes within the microstructure (see 2.1.1) resulting from cold working. It was observed that a certain strain e.g. size reduction due to cold rolling (see Figure 14) will reduce the grain size similar to an electromagnetic stirred alloy [16].

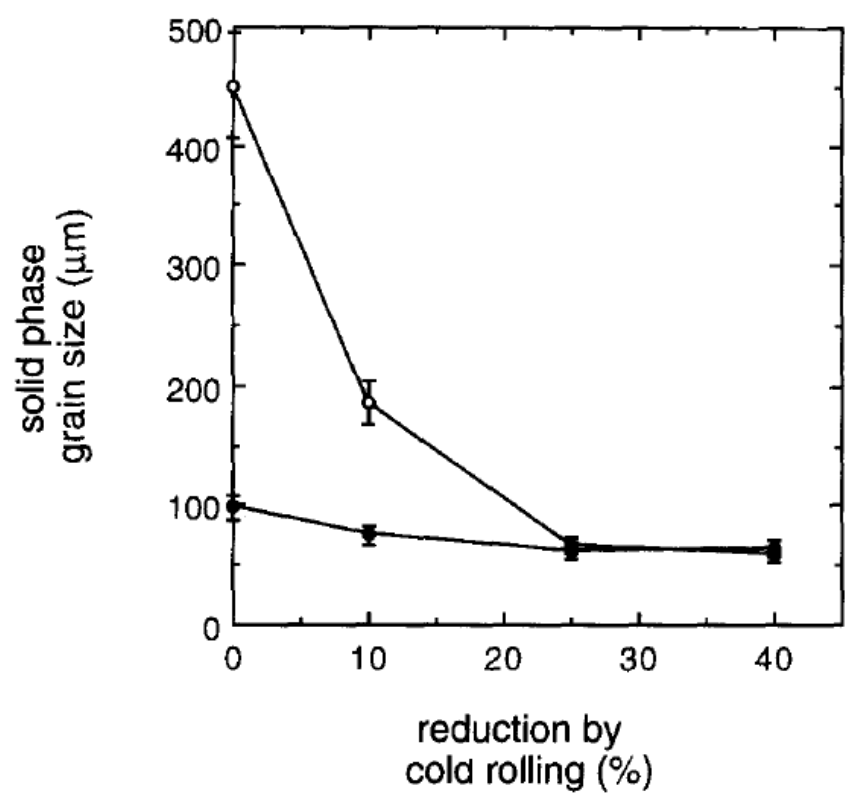

Figure 14 - Grain size of electromagnetic stirred $(\bullet)$ and cold rolled $(\circ)$ AlSi7Mg06 at $580^{\circ} \mathrm{C}$ and $30 \mathrm{~s}$ holding time.[16]

Loué and Suéry concluded that: "Cold working prior to partial remelting of AISi7Mg alloys allows the most rapid obtaining of a perfectly globular morphology of the solid phase, once the threshold deformation for recrystallization is surpassed. The final solid phase grain size is independent of the initial microstructure, coarsening while isothermal holding is slowed down when compared to the electromagnetically stirred alloy."

The patented process by Young et.al defines a four step process (see Figure 15) [45]:

1. Conventional casting of a dendritic alloy billet 
2. Hot working including size reduction by extrusion, rolling, forging or swaging at a below solidus temperature (typically $0.7^{\star} \mathrm{T}$ solidus Kelvin) to produce a striated and directional grain structure. Size reduction should be at minimum $10 / 1$ and suggested by the patent at a ratio between $1 / 19$ to about $1 / 60$.

3. Cold working as drawing, swaging, rolling, compression or upsetting equivalent to an at least $12 \%$ reduction.

4. Reheating the alloy into the semi-solid temperature range to achieve a liquid fraction between $0.05 \%$ to $0.8 \%$.

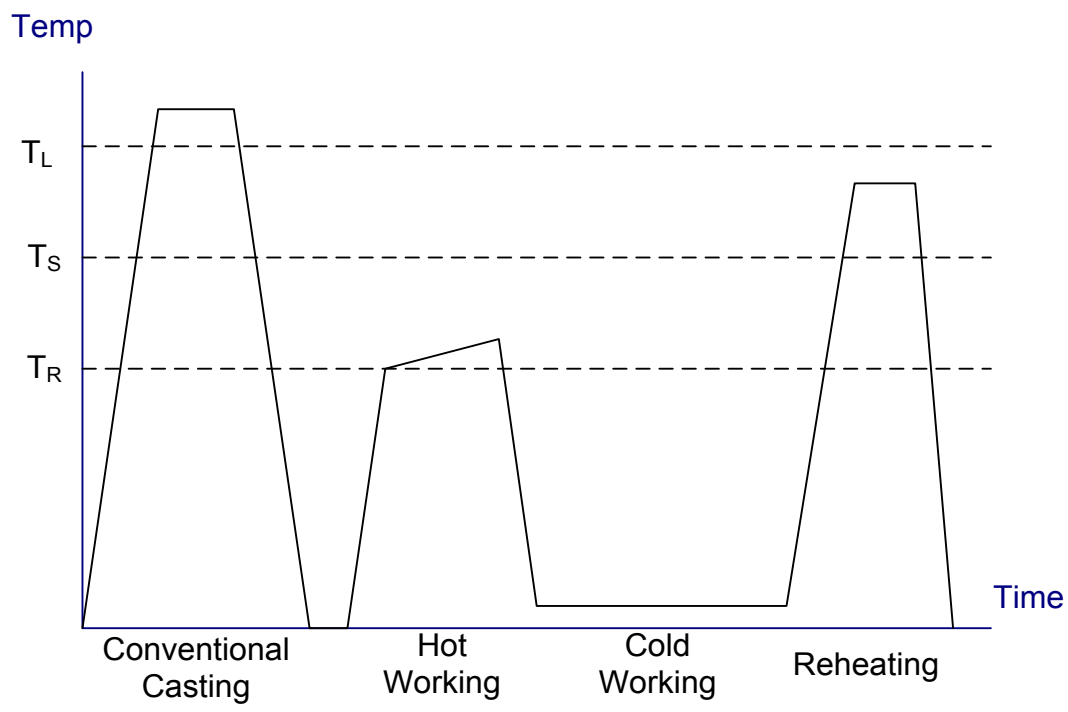

Figure 15 - Time - Temperature Profile of the SIMA Process[45]

The resulting grain size using the patented SIMA process is claimed to be below $30 \mu \mathrm{m}$. In his dissertation E. Tzimas [46] compared the SIMA, Spray Casting and the MHD process and concluded that the MHD compared to the other two processes exhibits a higher resistance to flow as a result of the morphology of the solid phase.

The SIMA process can be classified into the severe plastic deformation (SPD) that create significantly higher rates of dislocations. Equal Channel Angular Extrusion/Pressing (ECAP) is an SPD process with higher induced strain [47]. This process implies that a billet is extruded through two equal sized channels which intersect at an angle (see Figure 16) so that ideally the billet experiences simple 
shear without a change in the cross sectional area [48]. This process may result in anisotropic ${ }^{13}$ attributes.
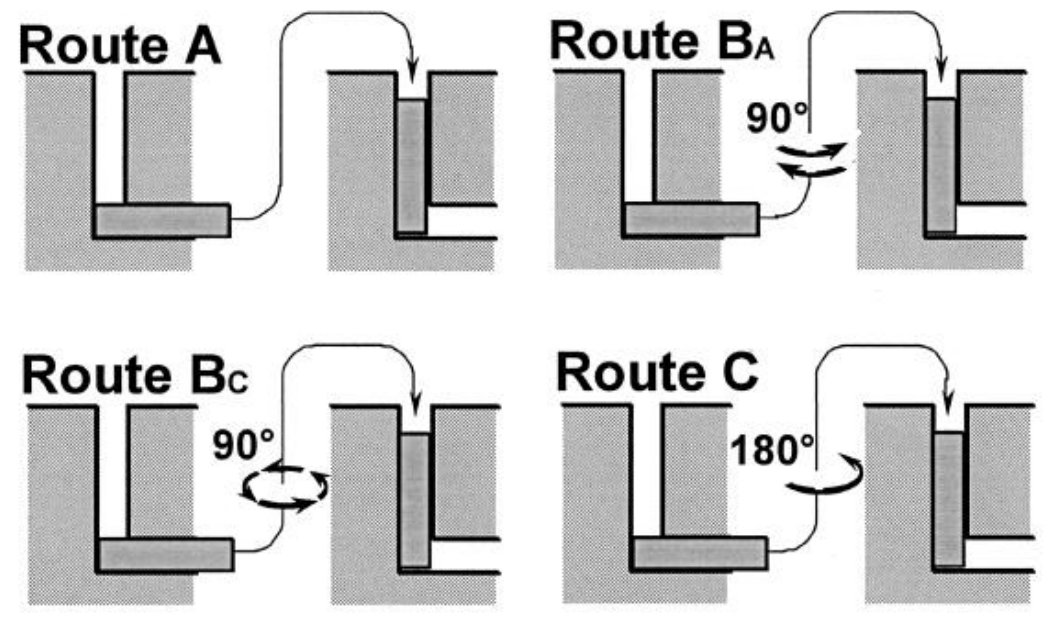

Figure 16 - Schematic illustration of ECAP processing routes with different or no rotation [48]

Jiang and Luo [49] used the ECAP as the strain induced step during the SIMA process. The "new SIMA" reduced the grain size from about $180 \mu \mathrm{m}$ to about $25 \mu \mathrm{m}$ at the semi-solid state of an AZ91D billet. Furthermore the "new SIMA" with ECAP resulted in increase of mechanical properties such as yield strength, ultimate tensile strength and elongation. Ashouri et.al [50] applied the ECAP process to A356 with one to four passes. The resulting grain size was below $90 \mu \mathrm{m}$ with a shape factor around 0.9 .

A continuous Equal Channel Angular Swaging (ECAS) process (Figure 17) for filament has been proposed by Groche et.al [51]. The swaging die performs an oscillating rotary movement resulting in a gradual severe plastic deformation. The copper specimen used had a reduced grain size from $16.3 \mu \mathrm{m}$ to $6.8 \mu \mathrm{m}$ with homogenous material attributes. The authors claim a reduce requirement of force as a result of the die exhibiting only short-term contact with the filament.

${ }^{13}$ Anisotropic is the directional dependency of attributes in case of EACP resulted by the direction of processing. Additional EACP passes with rotation can reduce the anisotropic result. 


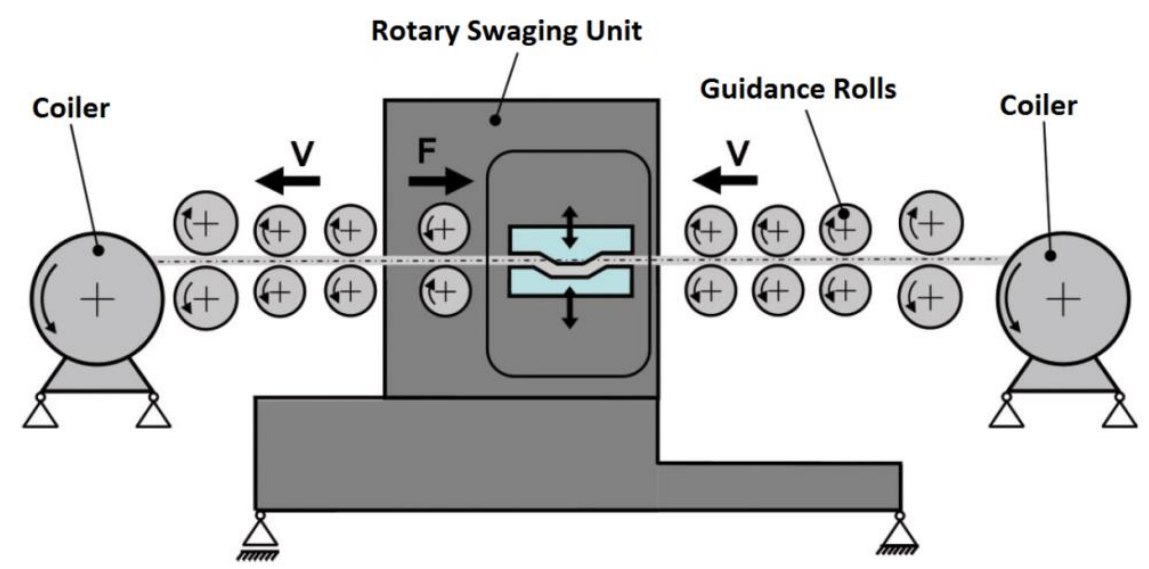

Figure 17 - Suggested continuous ECAS process for filament [51]

Chemical modification is a process by which additional elements other than the major alloy elements Al-Si-Mg or Cu or impurities e.g. Fe are added to the alloy [35]. This addition can be done during alloy production or in non thixocasting processes right before casting itself. Sauermann [52] and Noll [53] reviewed the design of Al alloys and enhancements to A356. Noll noted that chemical modification is a compromise between achievement of small grain size, spheroidization and fineness of eutectic areas. Strontium ( $\mathrm{Sr}$ ) acts as a eutectic modifier whereas Titanium and Boron are used as grain refinement.

\subsubsection{Conclusion on pre-process routes for semi-solid metal}

Semi-solid aluminum slurries can exhibit extremely different characteristics than ABS or similar plastics used in popular MEAM processing based on composition and pre-processing/history. It is essential to achieve a semi-solid slurry that exhibits a stable (in terms of time and temperature) viscosity so it can be extruded and deposited to form an object. The strain induced pre-processing route is already employed for producing an aluminum alloy filament via a drawing process. With initial extrusion molding from an ingot and then further hot and finishing cold drawing (instead of rolling) the process is very similar to the SIMA process reviewed above. It is assumed that a produced A356 filament already exhibits key attributes for semi-solid processing. Besides the potential composition changes e.g. for reduction of oxidation and further reduction of the grain size, additional changes to the pre-processing needs to be part of the research to find an adequate 
MEAM aluminum alloy filament. Recent research improves the strain induced process route through more advanced severe plastic deformation processes. Depending on the initial experiments this might lead to further options for improving the filament for the MEAM process.

\subsection{Additive Manufacturing}

The term Additive Manufacturing (AM) has been defined in 2009 by ASTM International as a "process of joining materials to make objects from 3D model data, usually layer upon layer, as opposed to subtractive manufacturing methodologies" [54].

The AM process can be separated into eight process steps (Figure 18) [55] transferring the part idea into a Computer Aided Design (CAD) file followed by the creation of an STL or newer $\mathrm{AMF}^{14}$ file. Optionally the STL or AMF file needs to be manipulated for repositioning, orientation change, segmentation or combination of objects(s), changing size to compensate for shrinkage and for other method or application specific reasons. After the completion of the STL/AMF file the machine needs to be set up e.g. changing layer size or material. The build process can typically run fully autonomously or with limited user interaction e.g. exchange of (support) material. After the object is built it must be removed from the build platform and, depending on the method, post-process removal of supports or unused material may be required. To finalize the part for use it might need some form of surface treatment e.g. polished. Some methods also require heat treatment when the resulting object is a "green" part for sintering. After those process steps the part is ready for use.

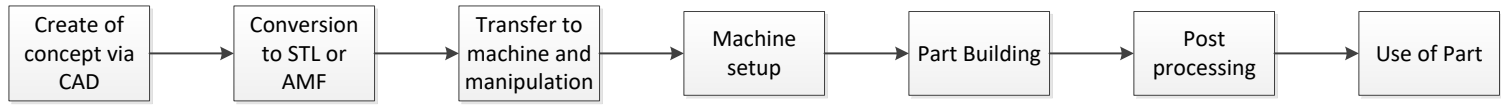

Figure 18 - Additive Manufacturing process chain [55]

\footnotetext{
${ }^{14}$ Improved file format
} 
Use may differ depending on the application for prototyping or end application. A well-known end application of AM technology is the creation of dental aligners by Align Technology [56].

\subsubsection{Variations of Additive Manufacturing}

Additive Manufacturing can be categorized by the raw material $[55,57]$ but this does not show the full complexity of the process categories. A better categorization method has been established by the ASTM International[54] through a combination of deposition and fusing types.

Table 4 - Advanced Manufacturing variations

\begin{tabular}{|c|c|c|}
\hline $\begin{array}{l}\text { ASTM F2792-12a } \\
\text { classifications [54] }\end{array}$ & Description [54] & $\begin{array}{c}\text { Example Technology } \\
\text { implementations }\end{array}$ \\
\hline Binder Jetting & $\begin{array}{l}\text { Liquid binder selectively } \\
\text { joining powder materials }\end{array}$ & ZPrinter \\
\hline $\begin{array}{l}\text { Direct Energy } \\
\text { deposition }\end{array}$ & $\begin{array}{l}\text { Focused thermal energy } \\
\text { fuses materials by melting as } \\
\text { they are being deposited }\end{array}$ & $\begin{array}{l}\text { Laser Engineered Net } \\
\text { Shaping (LENS) }\end{array}$ \\
\hline Material Extrusion & $\begin{array}{l}\text { Dispensed material } \\
\text { selectively through a nozzle } \\
\text { or orifice }\end{array}$ & $\begin{array}{l}\text { Fused Deposition Modeling }^{\circledR} \\
\text { (FDM), } \\
\text { Fused Filament Fabrication } \\
\text { (FFF) }\end{array}$ \\
\hline Material Jetting & $\begin{array}{c}\text { Droplets of build material are } \\
\text { selectively deposited }\end{array}$ & Stratasys PolyJet Process \\
\hline Powder Bed Fusion & $\begin{array}{l}\text { Thermal energy selectively } \\
\text { fuses regions of powder bed }\end{array}$ & $\begin{array}{l}\text { Selective Laser Sintering } \\
\text { (SLS), } \\
\text { Selective Laser Melting } \\
\text { (SLM), } \\
\text { Direct Metal Laser Sintering } \\
\text { (DMLS), } \\
\text { Electron Beam Melting } \\
\text { (EBM) }\end{array}$ \\
\hline $\begin{array}{c}\text { Vat } \\
\text { Photopolymerization }\end{array}$ & $\begin{array}{l}\text { Liquid photopolymer in a vat } \\
\text { is selectively cured by light- } \\
\text { activated polymerization }\end{array}$ & Stereolithography (SL) \\
\hline Sheet Lamination & $\begin{array}{l}\text { Sheets of material are } \\
\text { bonded to form an object }\end{array}$ & $\begin{array}{c}\text { Laminated Object } \\
\text { Manufacturing LOM } \\
\text { Ultrasonic Consolidation UC }\end{array}$ \\
\hline
\end{tabular}


The most common method for making metal parts is via powder bed fusion (see Figure 19 for installations). Other methods for metal part production include directed energy deposition, binder jetting and Ultrasonic Consolidation (UC) [58]. Recent research [57] reviewed the fatigue behavior of SLM ${ }^{15}$ produced metal parts which result in comparable dynamic behavior to conventionally processed stainless steel. Besides that the current trends are to create faster machines e.g. DMLS systems with up to $40 x$ within 4 years [59] and creation of large build volumes e.g. $200 \mathrm{~mm} \times 620 \mathrm{~mm} \times 620 \mathrm{~mm}$ [60].

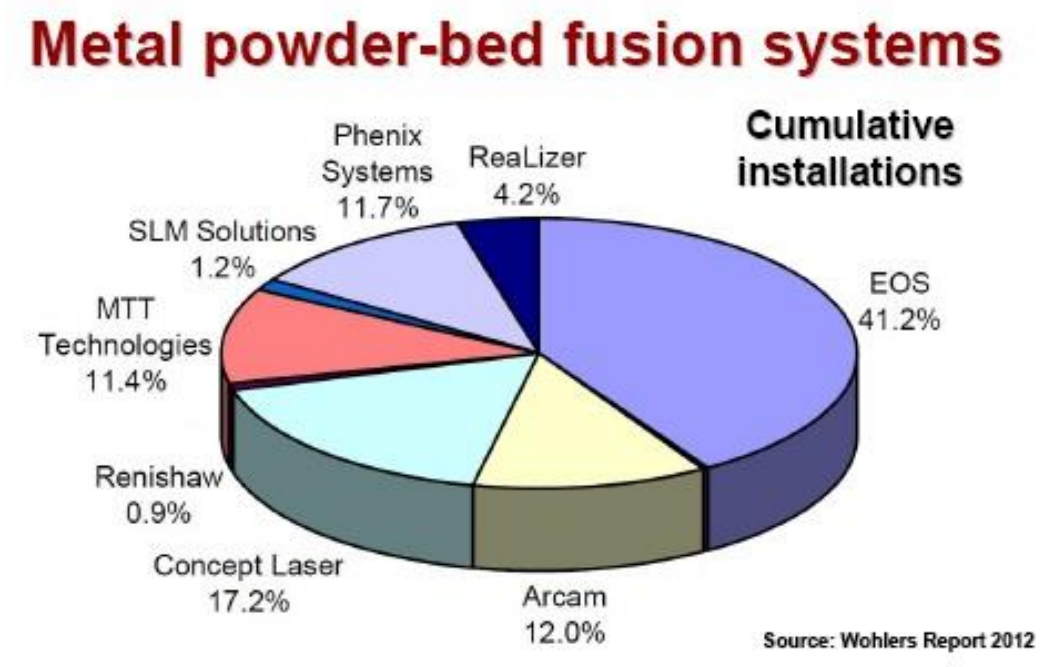

Figure 19 - Metal powder-bed system installations [61]

Further review of AM technology will be restricted to the MEAM as the dissertation focuses on the application of metal with MEAM.

\subsubsection{Material Extrusion Additive Manufacturing (MEAM)}

MEAM is an AM technology using a filament (currently ABS, polyphenylsulfone, polycarbonate) transported into a liquefier chamber and extruded through a nozzle tip (see Figure 20). The filament material is heated to a temperature where the viscosity of the material is sufficient for extrusion but stable enough to keep the form after extrusion.

\footnotetext{
${ }^{15}$ Powder-bed system with a laser as heat source (SLM) as compared to an electron beam as heat source $(\mathrm{EBM})$
} 
The filament material is used as a piston in its non-liquefied state pressing the liquefied material through the extrusion die. A screw or pinch roller system is used to push the filament into the liquefier and create the piston like pressure. The position between the base-plate and the nozzle is changed in in the $x$-y direction within a layer. When the layer is finished the distance between the base-plate and the nozzle is increase in the $z$ dimension by one layer thickness to extrude the next layer.

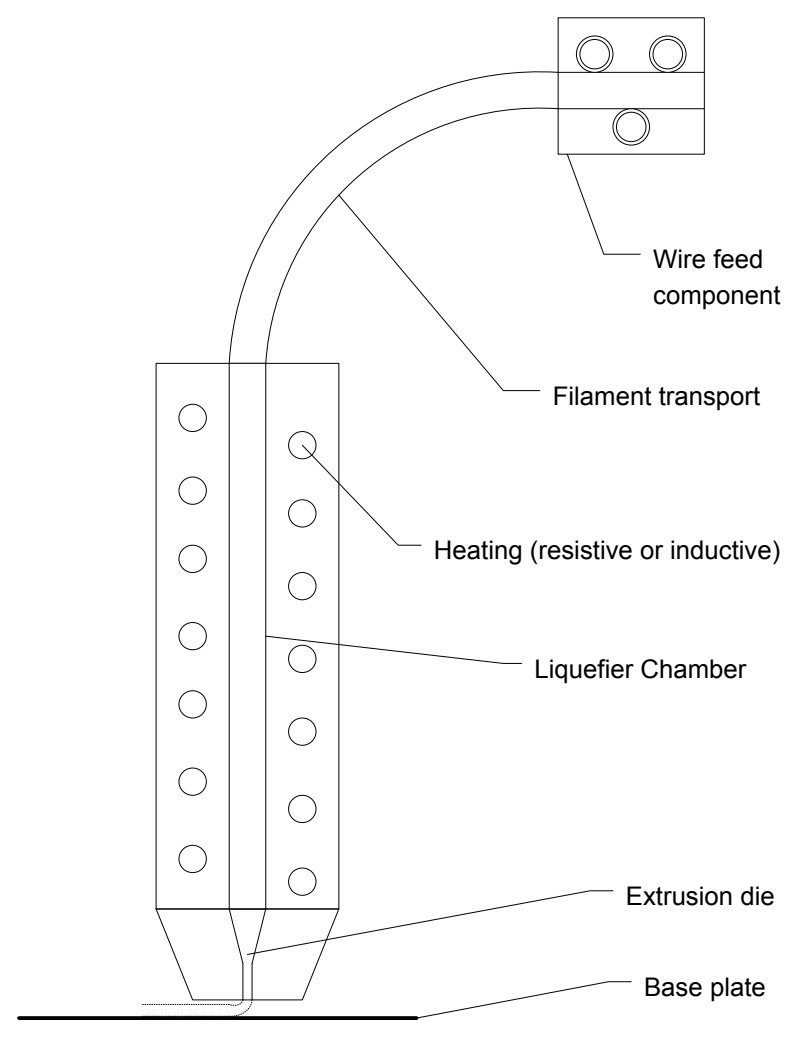

Figure 20 - Schematic of Fused Deposition Modeling

The possibilities for the mechanical implementation of the $x-y-z$ changes range from a complete stable base plate and full motion of the nozzle (common for most $\mathrm{CNC}$ machines), the complete stable nozzle with the base-plate performing all motions or a mixture in between. When extruding the next layer the remaining heat energy must be sufficient to allow the region of contact between the laid rods to bond. Additional areas of concern [55] are the 2D fill and 3D layer pattern, path control between a fill structure and boundary, start-stop problems and the use of support material. As this dissertations reviews the basic extrusion of semi-solid 
aluminum further literature review will be restricted to the filament transport, heating process, die extrusion process and heat transfer applicable to bonding. It is required to examine those topics for conventional current filament types and later adapt this knowledge for aluminum alloys.

\subsubsection{Filament transport}

When restricting the review to the transport of continuous filaments instead of other solid possibilities like pellets or powders [55] filament transport can be done via feed rollers or screw-feed. The standard design for welding machines using filament as well as $3 \mathrm{D}$ Printers is a pinch roller feeder such as that used by a Stratasys uPrint machine [62]. For plastic filaments the feed rollers typically use teeth to increase the transmission of power from the rollers to the filament. To maximize power transmission and also improve the flexibility of used feedstock the fabbster 3D printer uses corrugated or "teethed" sticks (see Figure 21) and calls this "Stick Deposition Molding" [63].

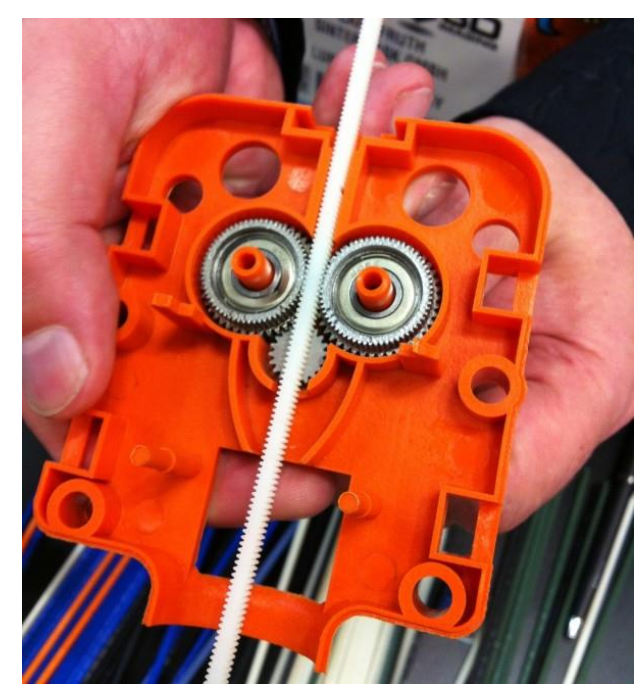

Figure 21 - fabbster teethed stick feed [64]

Each stick has a female and male side that can be connected so that the sticks form a continuous filament. (see Figure 22) The storage mechanism of these sticks connects the next available stick automatically at the time that the last one at the bottom is removed. 


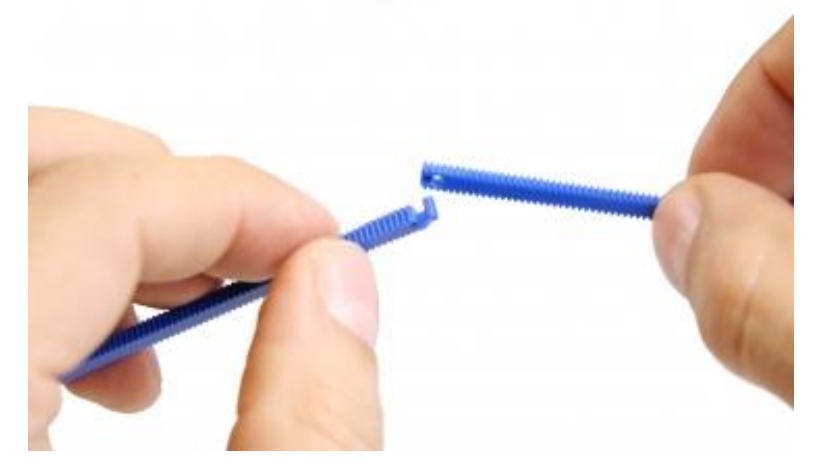

Figure 22 - Connecting of Fabbster sticks for form filament

Aluminum filament feeding devices are common in MIG welding machines. There are numerous patents that describe those mechanisms. Examples for the purpose of automation are the reduction of vibration [65] or the measurement of the wire speed [66]. The wire feeding device defines the force that the solid wire will apply to the semi-solid slurry in the heating channel, similar to a piston. Assuming the force applied to the wire from the feeding rollers is tangential then the force of the wire/piston $(F)$ can be calculated using the motor torque $(M)$, radius of the feeding rollers ( $r$ ), a slip percentage of the feeding rollers (s), the gear ratio between the motor and the feeding rollers $(\mathrm{R})$ and efficiency of the gear $\left(\eta_{G}\right)$.

$$
F=(1-s) * R * \eta_{G} * \frac{M}{r}
$$

When using pinch rollers the shape of the filament touching surface depends on the type of filament. It is suggested [67] to use a $V$ shape for steel filaments (Figure 23 - 1) and a $U$ shape for aluminum filaments (Figure $23-2$ ). If the wire feeder is not directly attached to the nozzle a wire liner needs to be used which will result in additional friction for the filament. Such friction forces will be up to 4-5 times higher [68] for wire liners in a curved or even looped geometry. Padilla, et al. [68] measured friction forces for wire liners of $0.75 \mathrm{~m}-3 \mathrm{~m}$ and aluminum filament of about $2 \mathrm{~N}$ to $8 \mathrm{~N}$ depending on wire liner geometry and liner material (Nylon/Teflon). 


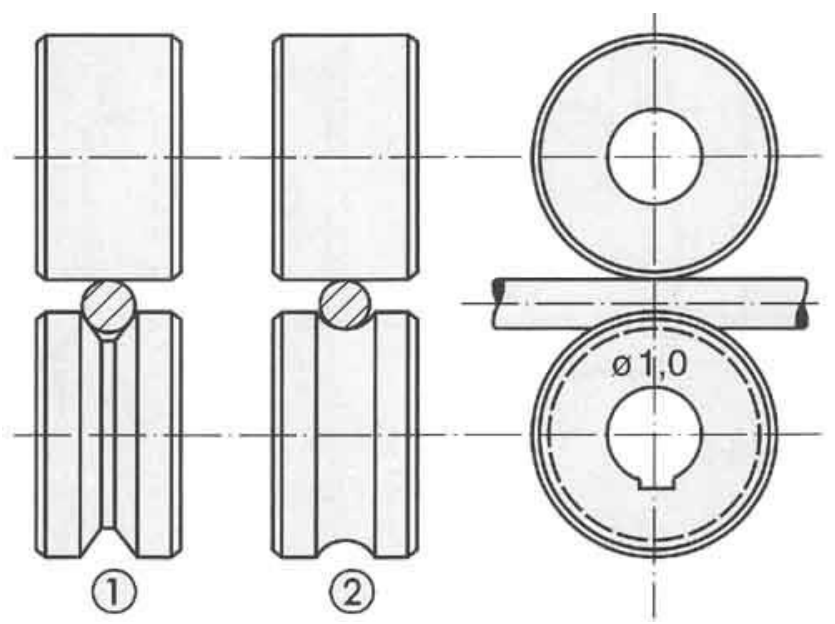

Figure 23 - filament pinch roller types 1-steel, 2-aluminum [67]

Go et. al. [69] reviewed the pinch wheel feed of a standard FFF $3 d$ printer and identified the maximum force that can be applied at higher feed rates.
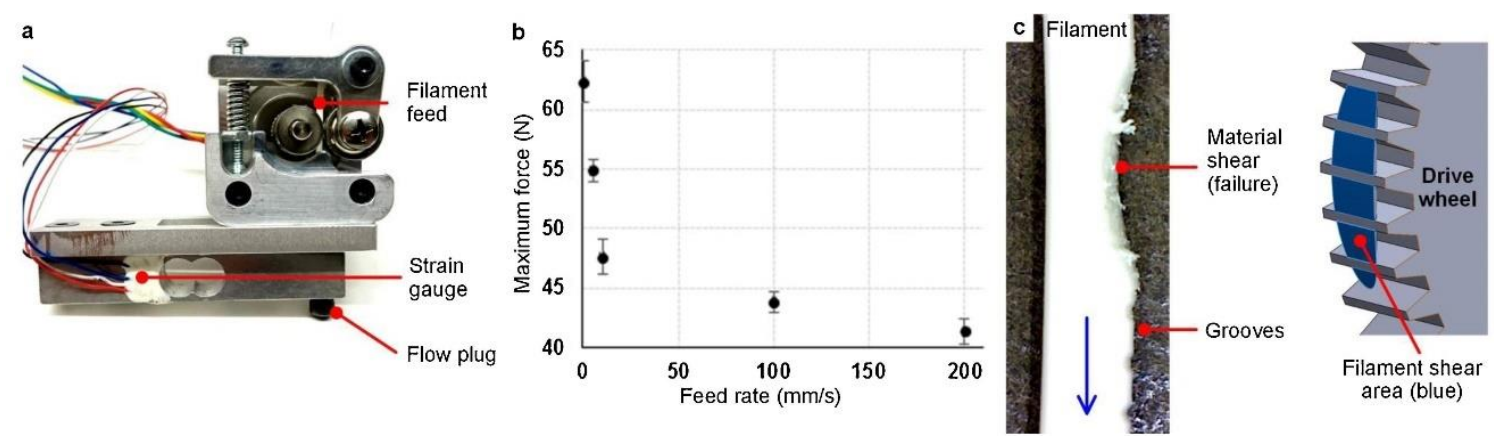

Figure 24 - Filament feed force and shear a) nozzle measurement b) feed rate vs force c) shear failure [69]

Based on the findings of the review a new process has been developed for filament feeding to allow high speed 3D printing FastFFF [70]. To allow a higher force to be applied to the filament similar to the "Stick Deposition Molding" [63] a nut-feeder has been added to the extruder. Filament has been cut with a standard 4-40 thread adding a triangular profile as a preparation and then pushed forward by the nut (see Figure 25). For a single threat cut into the filament Go et.al. were able to apply up to $109 \mathrm{~N}$ axial force before the shear failure occurred. Go et.al. commented that an improvement could be made by working on the thread profile and coating of the nut. 


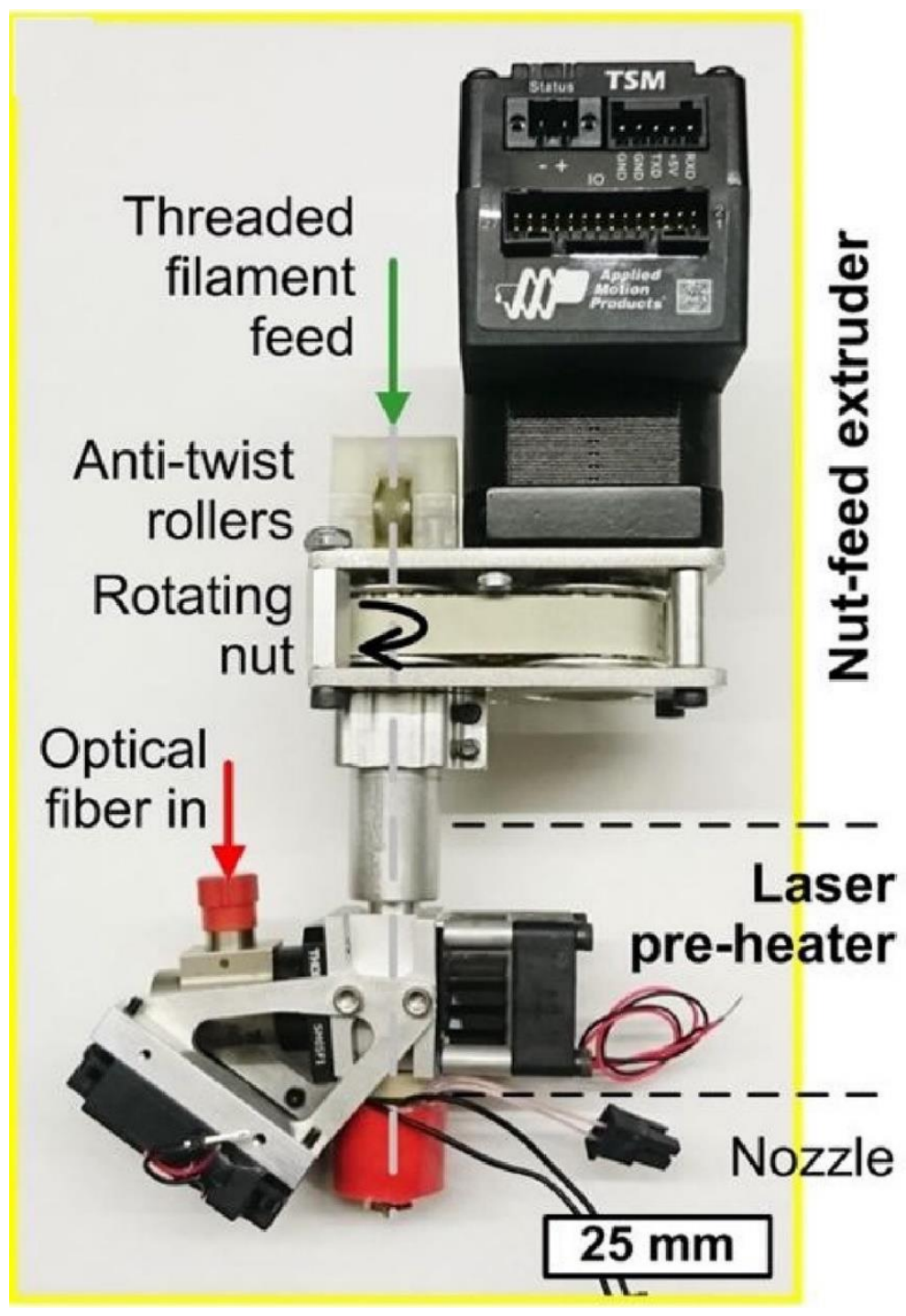

Figure 25 - FastFFF Extruder with nut feeder and laser pre-heater [70]

\subsubsection{Heating process}

To process the aluminum alloy in a semi-solid state the filament has to be heated to a very exact predefined temperature range. For aluminum alloy filament two practical processes are possible [71]: applying heat through conduction in a heating channel which is typically controlled via resistive heating or the induction of heat through changes in a magnetic field penetrating the filament. Conductive/resistive heating is common in MEAM 3D Printers whilst inductive heating is common in heating processes for thixotropic aluminum casting/forging. Advantage of resistive heating is its lower complexity of the equipment compared to induction heating. Induction heating allows a faster change of the energy feed 
and allows temperature control by indirect measurement via the induction coil [72]. Independent of the heating process the filament requires a finite energy $(\Delta Q)$ to heat up from the initial (room or pre-elevated) temperature $\left(T_{1}\right)$ to the semi-solid extrusion temperature $\left(T_{2}\right)$. This energy can be calculated using the mass $(m)$, the specific heat $\left(c_{p}\right)$ and the temperature difference $\left(T_{2}-T_{1}\right)$ as follows [73]:

$$
\Delta Q=m c_{p}\left(T_{2}-T_{1}\right)
$$

Compared to the MEAM process with ABS, when using an aluminum alloy about 5 -times more energy is required to get to the semi-solid state, due to:

- the higher temperature difference $\left(565^{\circ} \mathrm{C}\right.$ vs. $\left.210^{\circ} \mathrm{C}\right)$,

- higher density $\left(2.67 \mathrm{~g} / \mathrm{cm}^{3}\right.$ vs. $\left.1.04 \mathrm{~g} / \mathrm{cm}^{3}\right)$

- lower specific heat capacity $\left(0.963 \mathrm{~J} / \mathrm{Kg}^{*} \mathrm{~K}\right.$ vs. $\left.1.3 \mathrm{~J} / \mathrm{Kg}^{*} \mathrm{~K}\right)$.

To further analyze the conductive heating process the general heating equation (see Equation 2.29) can be derived using conservation of energy as a basis. The thermal energy flowing into a system or control volume $\left(E_{\text {in }}\right)$ minus the energy flowing out of the system ( $\left.\mathrm{E}_{\mathrm{ou}}\right)$ plus the energy created in the system $\left(\mathrm{E}_{\mathrm{g}}\right)$ equals the change of the energy stored $\left(\Delta \mathrm{E}_{\text {st }}\right)$ in the system [73]:

$$
\Delta E_{\text {st }}=E_{\text {in }}-E_{\text {out }}+E_{g}
$$

This equation can be used to show the rate of energy changes as follows[73]:

$$
\dot{E}_{s t} \equiv \frac{d E_{s t}}{d t}=\dot{E}_{\text {in }}-\dot{E}_{\text {ou }}+\dot{E}_{g}
$$

Using this equation a general heat equation can be derived using a control volume model (see Figure 26) also known as heat diffusion equation [73]:

$$
\frac{\partial}{\partial x}\left(k \frac{\partial T}{\partial x}\right)+\frac{\partial}{\partial y}\left(k \frac{\partial T}{\partial y}\right)+\frac{\partial}{\partial z}\left(k \frac{\partial T}{\partial z}\right)+\dot{q}=\rho c_{p} \frac{\partial T}{\partial t}
$$




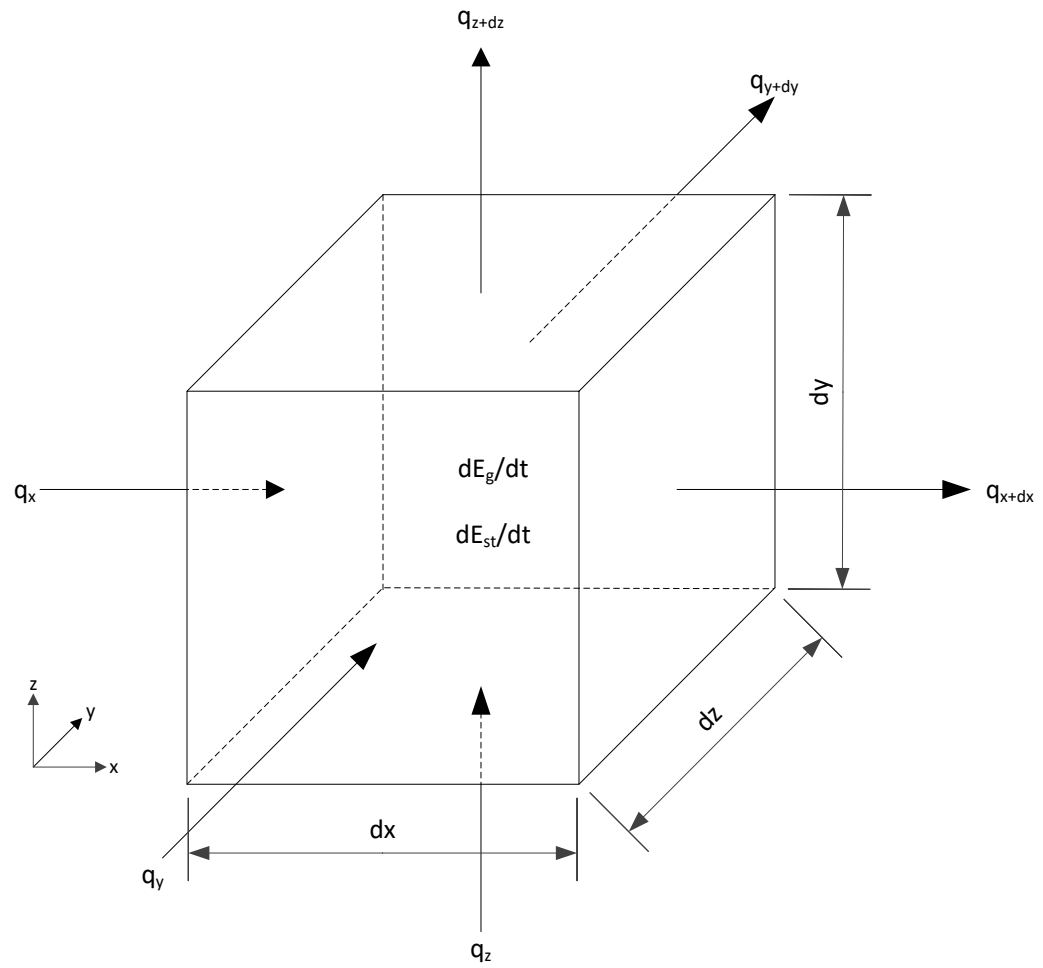

Figure 26 - Differential control volume for conduction analysis [73]

For a cylindrical control volume as observed in a filament the following heat equation can be achieved by replacing coordinates $x$ and $y$ with $r$ and $\phi$ [73]:

$$
\frac{1}{r} \frac{\partial}{\partial r}\left(k r \frac{\partial T}{\partial r}\right)+\frac{1}{r^{2}} \frac{\partial}{\partial \phi}\left(k \frac{\partial T}{\partial \phi}\right)+\frac{\partial}{\partial z}\left(k \frac{\partial T}{\partial z}\right)+\dot{q}=\rho c_{p} \frac{\partial T}{\partial t}
$$

Using this symmetric cylindrical approach for filament heating and assuming the heater and the filament do not have temperature gradients in the $\phi$ direction then it can be assumed that the heat transfer in the $\phi$ direction is zero [74]:

$$
\frac{1}{r^{2}} \frac{\partial}{\partial \phi}\left(k \frac{\partial T}{\partial \phi}\right)=0
$$

That reduces the heat equation to a 2 dimensional problem which in the case of billet heating could be reduced even further when assuming that the heat transfer in the z-direction is also zero[74]. In the case of a continuous aluminum filament this cannot be assumed which leads to the 2-dimensional heat equation 2.32: 


$$
\frac{1}{r} \frac{\partial}{\partial r}\left(k r \frac{\partial T}{\partial r}\right)+\frac{\partial}{\partial z}\left(k \frac{\partial T}{\partial z}\right)+\dot{q}=\rho c_{p} \frac{\partial T}{\partial t}
$$

A heating channel with a filament can be seen as a composite from a heat transfer perspective. The contact area between the heating channel and the filament results in an additional thermal contact resistance due to surface roughness and air gaps [73] (see Figure 27).
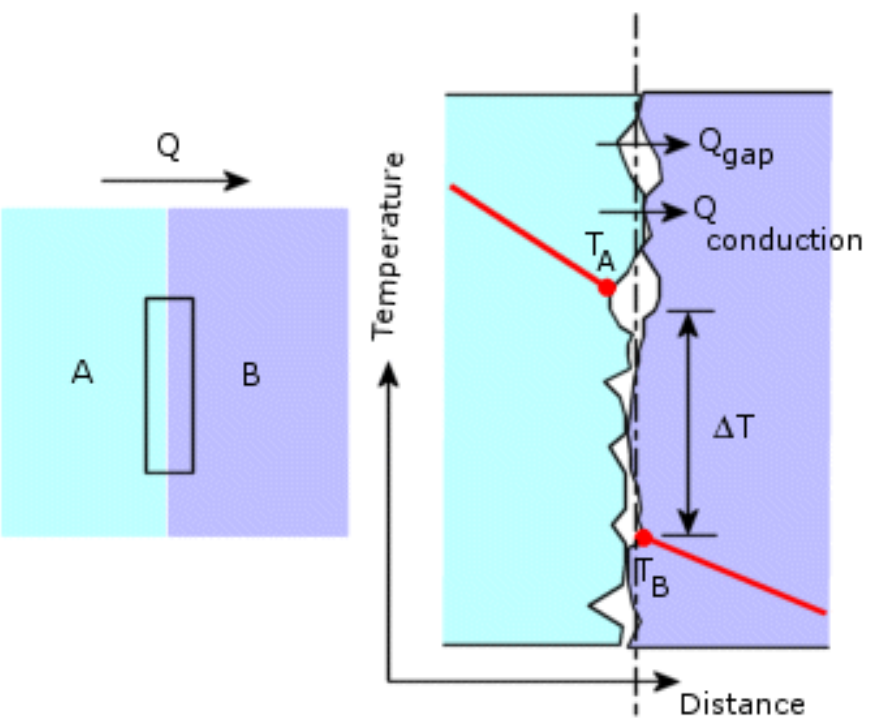

Figure 27 - Temperature drop due to thermal contact resistance [75]

Such contact resistance can be reduced through pressure but in the case of the heating channel would increase the friction of the filament. The contact resistance $\left(R_{t, c}^{\prime \prime}\right)$ can be determined by using the temperature difference at each composite wall and the heat flux $\left(q_{x}^{\prime \prime}\right)[73]$ :

$$
R_{t, c}^{\prime \prime}=\frac{T_{A}-T_{B}}{q_{x}^{\prime \prime}}
$$

Table 5 - Typical conductance with normal surface finish and moderate preasure (1 to $10 \mathrm{~atm}$ ) [75]

\begin{tabular}{|c|c|}
\hline Contacting Faces & Interfacial Conductance $\left(\mathrm{h}_{\mathrm{c}}\right)\left(\mathrm{W} / \mathrm{m}^{2} \mathrm{~K}\right)$ \\
\hline Iron/aluminum & 45,000 \\
\hline Copper/copper & $10,000-25,000$ \\
\hline Aluminum/aluminum & $2200-12000$ \\
\hline Stainless steel/stainless steel & $2000-3700$ \\
\hline Stainless steel/stainless steel & $200-1100$ \\
\hline
\end{tabular}




\begin{tabular}{|l|l|}
\hline (evacuated gaps) & \\
\hline Ceramic/ceramic & $500-3000$ \\
\hline
\end{tabular}

Using the heat transfer rate $q$ the thermal contact resistance can be calculated using an interfacial conductance $\left(h_{c}\right)$ and contact area $(A)$ [75]:

$$
R_{t}=\frac{1}{A * h_{c}}
$$

Typical interfacial conductivity values are listed in Table 5 [75].

In the case of a significant air gap (inert gas) between the filament and the nozzle wall, heating needs to be calculated as transfer from the heating nozzle to air and then from air into the filament. From the melting front downwards the gap can be assumed as closed due the piston pressure resulting from the wire feed [71].

Induction heating creates indirect closed loop current flow (known as eddy current) via an alternating magnetic field generated by an induction coil [76]. The workpiece itself must be conductive to allow the eddy current to flow, which is the reason that direct filament heating is not possible for plastic (e.g. ABS). Instead induction heating for the nozzle which subsequently heats up the plastic filament would be possible [77]. Through the electrical resistance in the workpiece the energy of eddy currents is transferred into heat, also known as Joule heating. In cases of magnetic materials, the heat in the workpiece is also generated by magnetic hysteresis losses. As aluminum and its alloys do not show significant magnetism the details of that influence are omitted.

When working with alternating current flows/magnetic fields the tendency of the current to flow in the outer area of a wire is known as the skin effect. This is due to the electromagnetic field which is created by the eddy currents and directed against the initial electromagnetic field of the induction coil. This field is largest at the center of the workpiece and pushes the currents to the outer (skin) area of the workpiece (see Figure 28). The size of the skin area is described by the Skin or Penetration Depth $(\delta)$. 


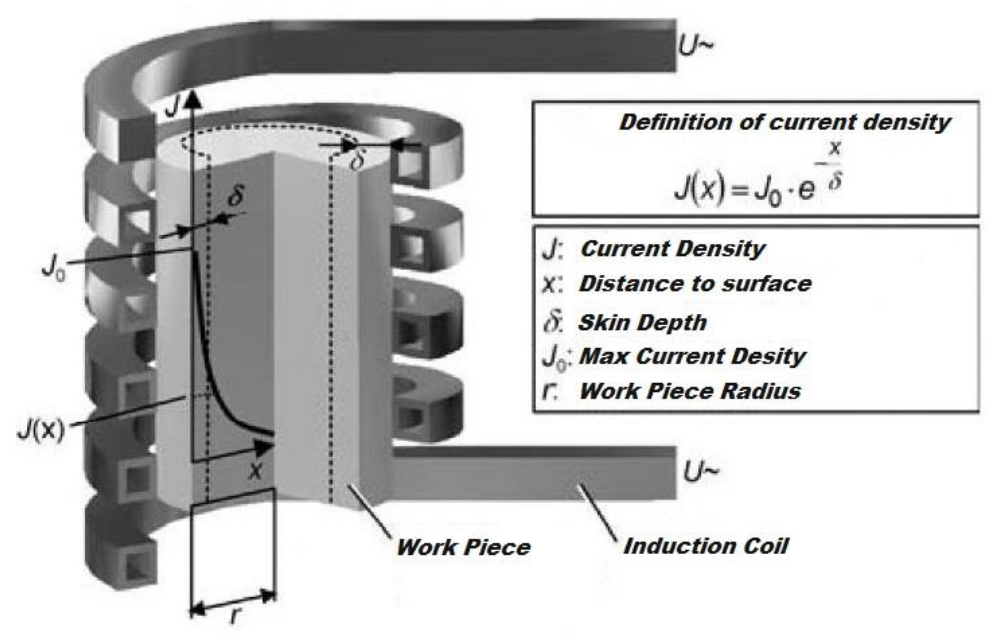

Figure 28 - Induction Heating and Skin Depth [76]

This skin depth for symmetric bodies with significant conductivity can be estimated by:

$$
\delta=\sqrt{\frac{2}{\sigma \mu \omega}}
$$

with $\sigma$ representing the electrical conductivity, $\mu$ the magnetic permeability and $\omega$ the angular frequency of the magnetic field (with $\omega=2 \pi f)$. The current density $(J)$ at a depth $x$ can be calculated using the skin depth $(\delta)$ and the maximum current density $\left(\mathrm{J}_{0}\right)$ at the surface of the work piece (see also Figure 28):

$$
J(x)=J_{0} * e^{-\frac{x}{\delta}}
$$

This results in that at the depth of $\delta=1$ the current density is reduced to $1 / \mathrm{e}$ or $37 \%$ and about $86 \%$ of the power has been induced in this skin area. To achieve sufficient induction effectiveness the diameter of the work piece needs to be at least 3-4 times larger than the skin depth [78] (see Figure 30). The skin depth can be controlled using the frequency of the magnetic field as the other factors are material dependent (see Figure 29). In case of a $1 \mathrm{~mm}$ radius filament where the skin depth should be $1 / 4$ of the radius the frequency should be approximately $400 \mathrm{KHz}$ as per Skin Depth Monogram [79]. 


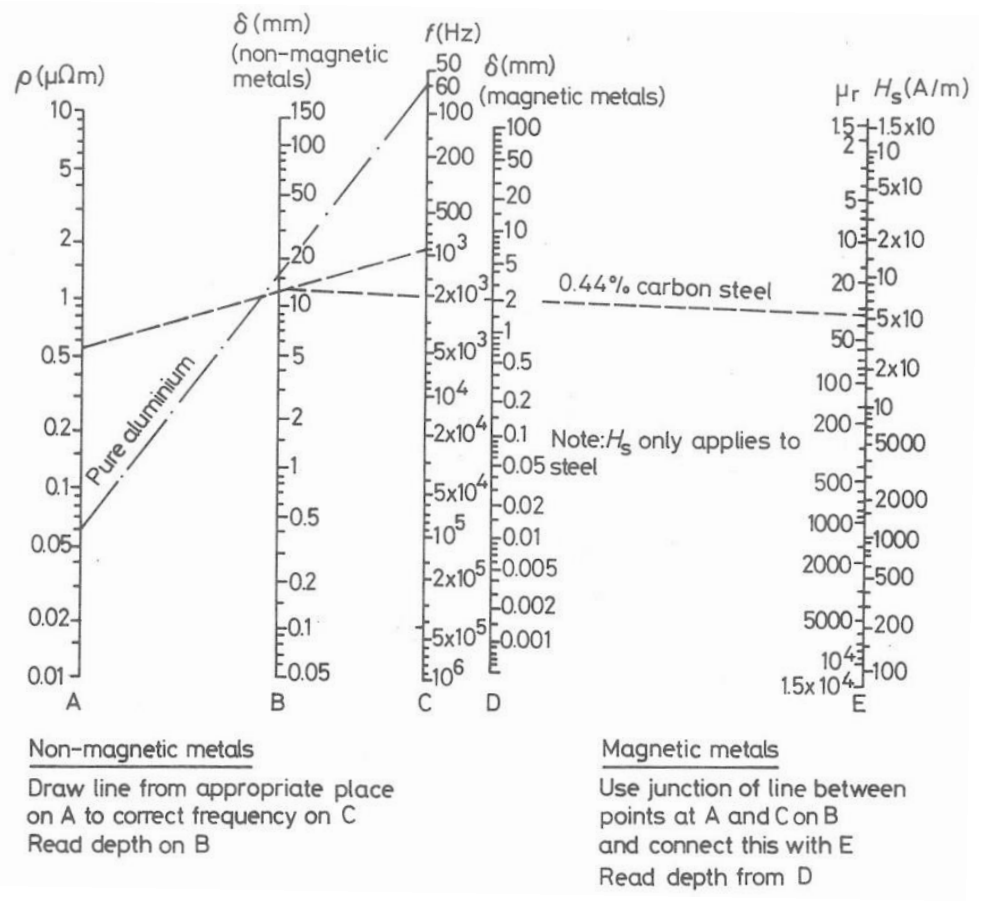

Figure 29 - Skin Depth Monogram [79]

As such the effectiveness has to be compromised with non-uniform heating from surface to center of the work piece. Therefore, the induction heating process is always a combination of electrical induced heat and heat transfer in the work piece which is described by the same heat equation 2.32 (using the simplified 2 dimensional heat equation) as above shown for resistive heating. In cases of billet heating to the semi-solid state the power is step wise reduced to allow a through heated billet with no significant temperature gradient. 


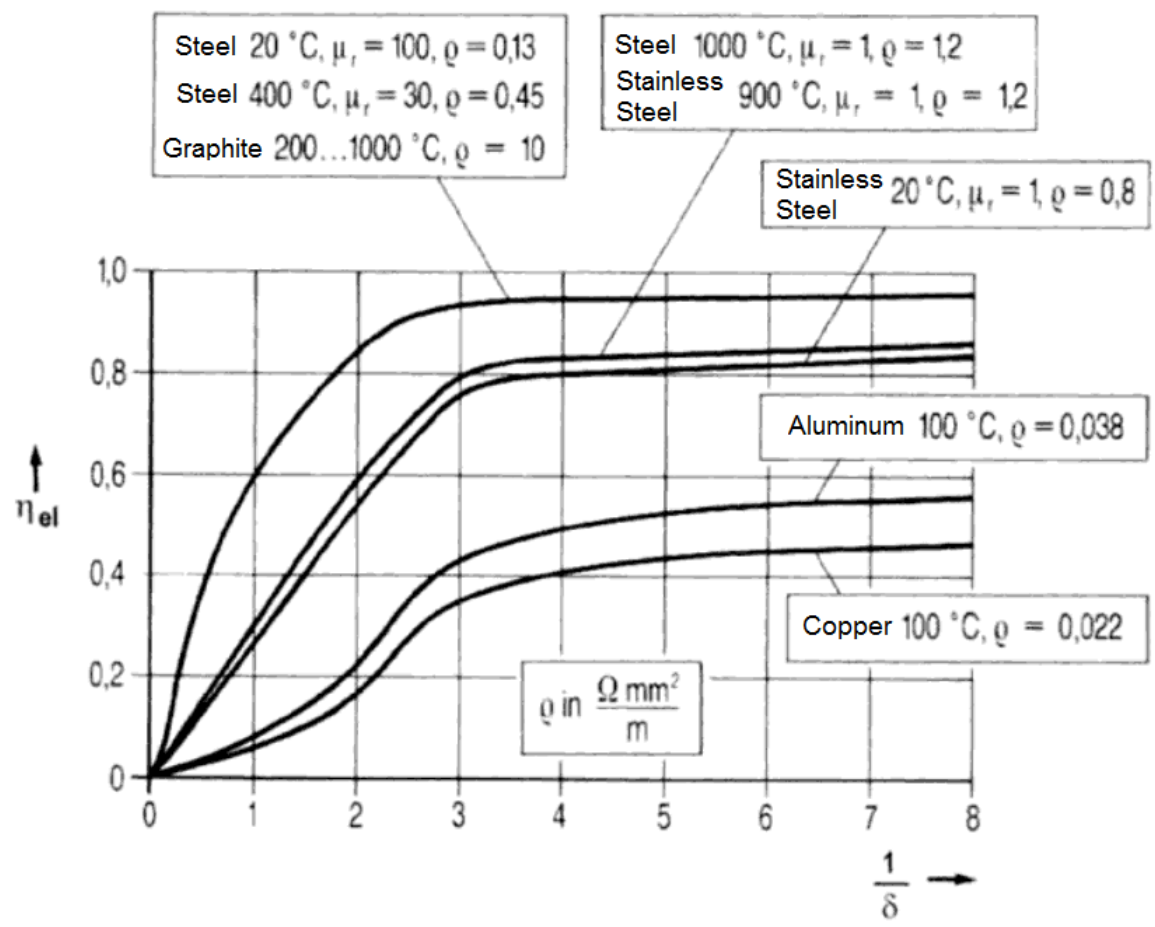

Figure 30 - Inductor-effectiveness per diameter skin depth ration (adapted from [80])

\subsubsection{Die extrusion process}

A nozzle in the MEAM Process could be seen as a pipe for a shear thinning fluid. At the heating channel a melt front can be found where the filament changes from solid to a semi-solid or slurry. This could be seen as a piston driving the semi-solid fluid through an extrusion die and can be compared to a direct hot extrusion still recognizing that the extrusion temperature would be lower than in a semi solid case. In case of a MEAM machine the heating zone is typically an axisymmetric cylindrical channel with a radius $R_{1}$ and a respective cross section $A_{1}$. The billet or in case of MEAM the semi-solid filament is pressed through a die with a orifice area $A_{2}$ and a die angle $\alpha$ (see Figure 31). 


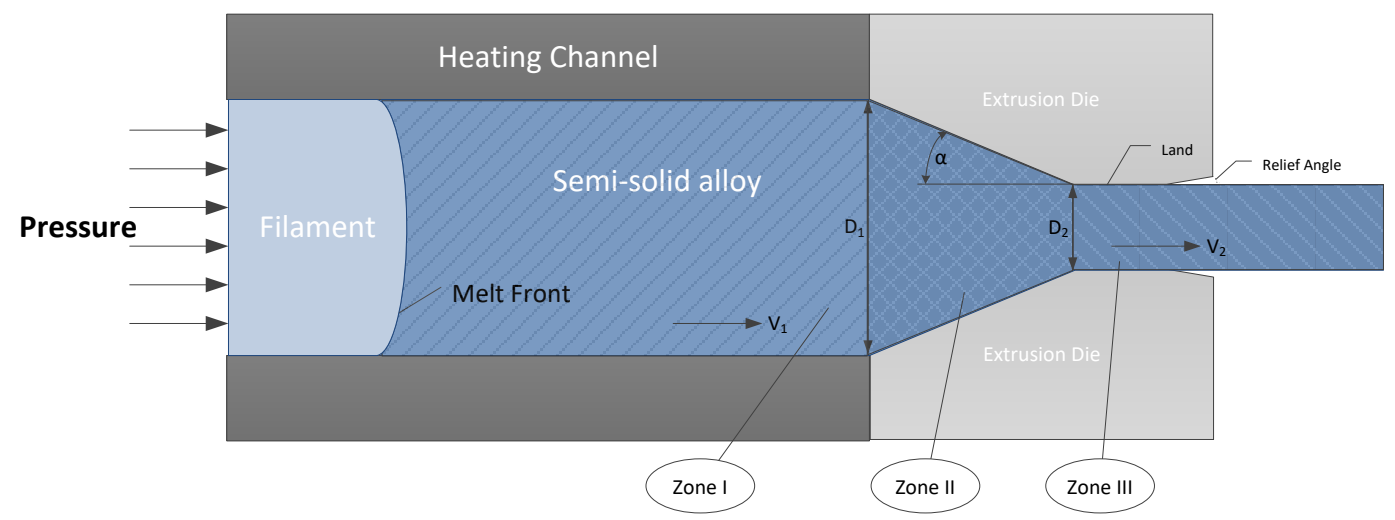

Figure 31 - Extrusion Principle (adapted from [81], [82] and [83])

For an ideal fluid without friction or a turbulent flow the pressure in the extrusion die would drop due to the conservation of momentum [84] as the velocity of the fluid will increase by the Extrusion Ratio R [81]:

$$
R_{e x}=\frac{D_{1}^{2}}{D_{2}^{2}}=\left(\frac{r_{1}}{r_{2}}\right)^{2}=\frac{A_{1}}{A_{2}}
$$

Based on this ratio the velocity can be calculated [81]:

$$
V_{2}=V_{1} * R_{e x}=V_{1} *\left(\frac{D_{1}}{D_{2}}\right)^{2}
$$

Compared to the metal extrusion below the liquidus temperature the activation energy in the convergence of the die only relates to the Bingham behavior of the semi-solid metal. In case of hot extrusion the extrusion force can be calculated using the initial area $\left(\mathrm{A}_{1}\right)$, a temperature based extrusion constant $\left(k_{c}\right)$ and the above calculated extrusion ratio [85]:

$$
F_{e x}=A_{1} k_{c} \ln R_{e x}
$$

Expected extrusion constants examples at different temperature are shown in Figure 32.

As the initial area multiplied by the pressure would result in the extrusion force, the term $k_{c} l n R_{e x}$ can be seen as the expected pressure drop in the extrusion system. 


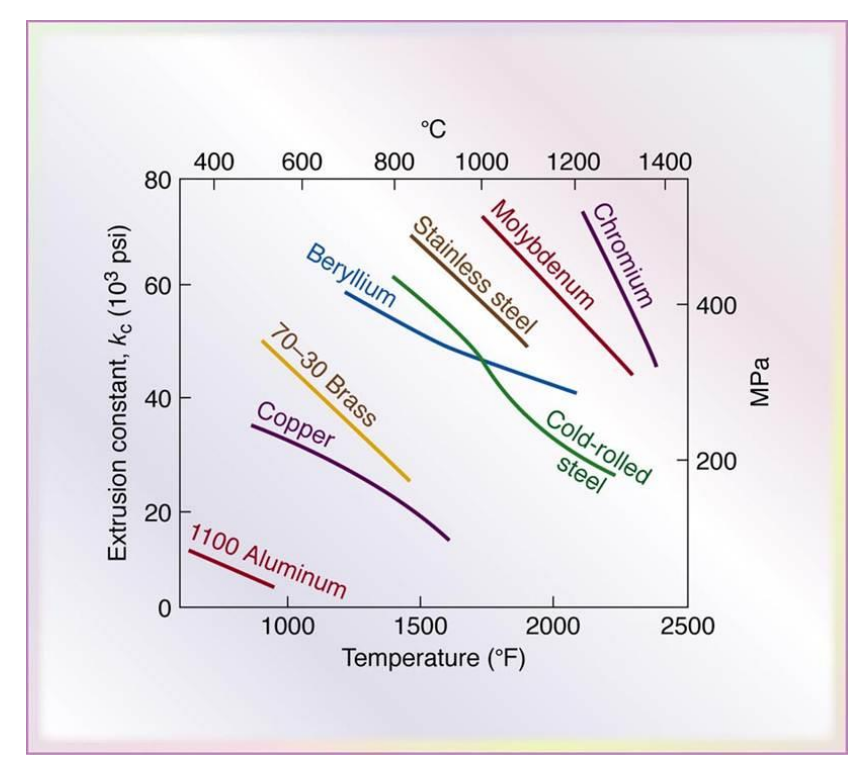

Figure 32 - Extrusion constant for various metals at different temperatures [85]

An assumed equivalence to the pressure drop might be found in the extrusion of shear thinning plastic as also used in conventional MEAM technology. The shear stress vs. strain relationship is modeled slightly differently than in section 2.1 .5 by transforming the consistency factor $\mathrm{k}$ and power law index $\mathrm{n}$ as follows [86]:

$$
k=\phi^{-\frac{1}{m}} \text { and } n=\frac{1}{m}
$$

This results in the following equations for shear stress $(\tau)$, shear rate $(\dot{\gamma})$ and dynamic viscosity $(\mu)$ during a steady state operation [86]:

$$
\begin{gathered}
\tau=\left(\frac{\dot{\gamma}}{\phi}\right)^{\frac{1}{m}} \\
\dot{\gamma}=\frac{8(m+3) \dot{Q}}{\pi D^{3}} \\
\mu=\left(\frac{\dot{\gamma}^{1-m}}{\phi}\right)^{\frac{1}{m}}
\end{gathered}
$$

Pressure drop in zones I, II \& III (see Figure 31) can be calculated as follows [86] using the length of zones I \& II $\left(L_{1}, L_{2}\right)$ where $L_{1}$ depends on the location of the melt front inside the heating channel: 


$$
\begin{gathered}
\Delta p_{\text {Zone I }}=\left[\frac{(m+3)\left(2 L_{1}\right)^{m} \dot{Q}}{\pi\left(\frac{D_{1}}{2}\right)^{m+3} \phi}\right]^{\frac{1}{m}} \\
\Delta p_{\text {Zone II }}=\frac{m}{6 \tan \left(\frac{\alpha}{2}\right)}\left[\frac{(m+3) 2^{m+3} \dot{Q}}{\pi \phi}\left(\frac{1}{D_{1}^{3}}-\frac{1}{D_{2}^{3}}\right)\right]^{\frac{1}{m}} \\
\Delta p_{\text {Zone III }}=\left[\frac{(m+3)\left(2 L_{2}\right)^{m} \dot{Q}}{\pi\left(\frac{D_{2}}{2}\right)^{m+3} \phi}\right]^{\frac{1}{m}}
\end{gathered}
$$

The resulting pressure drop along the extrusion nozzle is [86]:

$$
\Delta p=\Delta p_{\text {Zone I }}+\Delta p_{\text {Zone II }}+\Delta p_{\text {Zone III }}
$$

Nevertheless, this model would not be an ideal fit since in Zone I the temperature of the semi-solid alloy increases in front of the melt front location until the nozzle temperature is met. In this area the viscosity is not just dependent on the shear rate but also significantly on the temperature and the solid/liquid fraction.

An model of the link between the semi-solid MEAM pressure drop and the resulting extrusion force was used by Finke [71], modifying the calculation of the pressure drop in the nozzle capillary $(\Delta P)$ from Paradies et al. [87]

$$
\begin{gathered}
V_{\text {cap }}=\frac{\int_{0}^{2 \pi} \int_{0}^{R} V_{z} r d r d \theta}{\int_{0}^{2 \pi} \int_{0}^{R} r d r d \theta}=\frac{n R_{\text {cap }}}{3 n+1}\left[\frac{\Delta P R_{\text {cap }}}{2 K l}\right]^{\frac{1}{n}} \text { resulting in } \\
\Delta P=\frac{2 K l}{R_{\text {cap }}}\left[\frac{V_{c a p}(3 n+1)}{n R_{\text {cap }}}\right]^{n}
\end{gathered}
$$

and the pressure drop $\left(P_{e x}\right)$ in the extrusion die from Blazynski [88]:

$$
P_{e x}=\bar{\sigma}\left(0.8+1.5 \ln R_{e x}\right)
$$


$R_{\text {cap }}$ represents the radius in the capillary, $\Delta P$ the pressure drop, $K$ and $n$ the shear thinning material constants $V_{\text {cap }}$ the velocity in the nozzle, $z$ the axial and $r$ the radial coordinate.

The resulting force $\left(F_{e x}\right)$ calculated by:

$$
F_{e x}=A_{1}\left(\Delta P+P_{e x}\right)
$$

was significantly (2.5-3 times) lower than the force values Finke [71] measured in a load cell mounted in the extrusion head during the extrusion of semi-solid $60 \mathrm{~Pb}$ $40 \mathrm{Sn}$ alloy at $195^{\circ} \mathrm{C}$. Finke explained the difference by not taking into consideration friction / pressure drop in the heating channel and possible different segregation behavior in the back extrusion experiment that was used to determine the material constant $(K)$, the power law index $(n)$ and flow stress $(\bar{\sigma})$. (see also section 2.4.3)

Another aspect of the extrusion is the die angle $(\alpha)$ that will change the behavior of the raw material that will be extruded. This includes the flow type (laminar or turbulent), the shear stress on the material in the die and the force required. If the die angle is large enough a dead zone will be formed that can typically be found in planar dies [89] (see Figure 33).

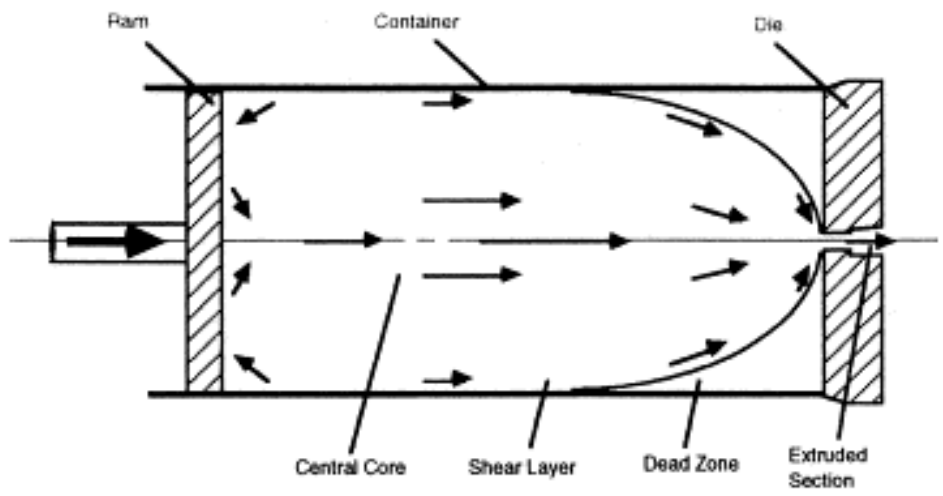

Figure 33 - Material flow in a planar extrusion die [89]

Orgéas et al. [26] showed the dead zone in a semi-solid extrusion experiment using A356. In contradiction to Finke's [71] observation Orgéas et al. identified a decreasing / lower pressure than expected by a no-slip Poiseuille flow. A thin 
eutectic layer of segregated liquid material (see liquid vein in Figure 34) was found in the capillary and in the converging area of the dead zone which leads to the assumption of a "...solid plug ('plug flow'), almost all of the shear rate being accommodated by this thin layer of liquid (i.e. replacement of the no-slip condition by a free-slip one)"[26].

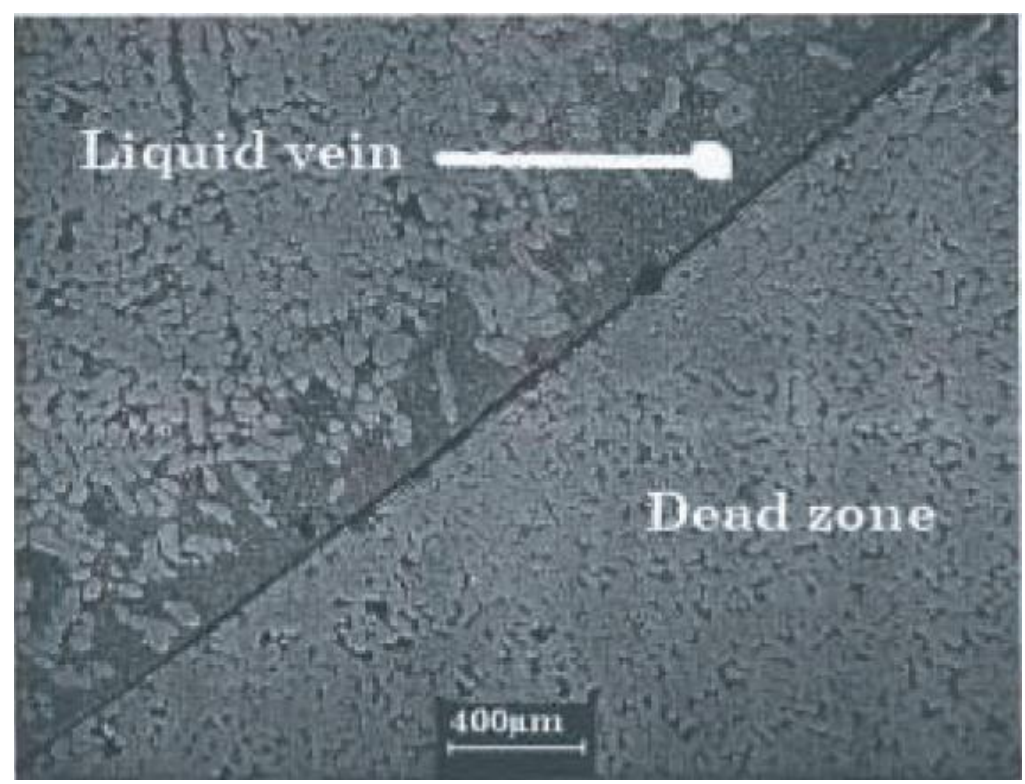

Figure 34 - Eutectic layer besides the dead zone during A356 extrusion [26]

Further publications on extrusion cover die design via modeling/simulation to result in a homogenous strain rate and best position for material overflow to limit semisolid segregation [90] or the reduction or minimization of distortion in the extruded material [82]. This could be applied to semi-solid MEAM as the current observed die designs are simple converging dies (e.g. $40^{\circ}$ die used in Finke's extrusion of semi-solid 60Pb-40Sn [71]).

\subsubsection{Heat Transfer for Layer Bonding}

Layer to layer bonding in AM is mainly determined by the remaining heat which activates the interface between the layers below and the newly deposited road. In addition, an oxidation skin and segregated material in the skin could change the bonding behavior in the case of a semi-solid aluminum alloy. There are several proposed interface heat transfer \& bonding potential models for the MEAM process. Yardimci et al. [91] described a cooling model that takes complex geometry into 
account. Cooling of the laid road will be influenced / delayed in case of either environment / build chamber temperature $\left(\mathrm{T}_{\infty}\right)$ or residual heat in the relevant neighbor roads $\left(T_{\text {neigh }}\right)$. These influences are covered in sink terms for convection losses $\left(S_{c}\right)$ and road interactions $\left(S_{l}\right)$ :

$$
\rho \frac{\partial q}{\partial t}=k \frac{\partial^{2} T}{\partial x^{2}}-S_{c}-S_{l}
$$

Filament material constants are represented by the density $(\rho)$, specific enthalpy $(\rho)$ and effective thermal conductivity. The process identifies the average crosssection temperature $(T)$ at the axial coordinate of the road $(x)$ and the time after deposition $(t)$. The sink terms are calculated using the road width (width), the convection coefficient $(h)$ and the ratio of the road element volume to its free surface area $\left(h_{e f f}\right)$ :

$$
\begin{gathered}
S_{c}=\frac{h}{h_{e f f}}\left(T-T_{\infty}\right) \\
S_{l}=\frac{k}{w_{i d t h^{2}}}\left(T-T_{\text {neigh }}\right)
\end{gathered}
$$

Using this temperature transition during the cooling process Yardimci et al. created a bonding potential number $(\phi)$ using the time the road spends above a critical bonding temperature $\left(T_{c}\right)$ with $\tau$ representing the integration variable:

$$
\phi=\int_{0}^{t}\left(T-T_{c}\right) d \tau
$$

Another model that has been created by Costa et al. [92] using a lumped capacitance approach. This approach allows an analytical solution for transient heat problems but requires the assumption that the heat gradient in the solid can be neglected when comparing it to the convection to an outside fluid. To ensure that this assumption holds true a test can be done by calculating the Biot number [73]. 


$$
B_{i}=\frac{h L_{c}}{k}
$$

This number will be small if the heat resistance of the solid is much less than the heat resistance of convection. The lumped capacitance approach can be applied if the condition: $B_{i}<0.1$ holds true [73].

Costa at al. [92] derived analytically the following equations to calculate the temperature at a time $\mathrm{t}$ :

$$
\begin{gathered}
T(t)=C_{1} e^{\left(\frac{-P b\left(a_{1}, a_{2}, a_{3}, a_{4}, a_{5}\right)}{\rho A C}-\frac{h_{c o n v} \phi}{\rho L C}\right)\left(t-t_{0}\right)}+Q\left(a_{1}, a_{2}, a_{3}, a_{4}, a_{5}\right) \\
+\frac{h_{c o n v} \phi A T_{E}}{P L b\left(a_{1}, a_{2}, a_{3}, a_{4}, a_{5}\right)}
\end{gathered}
$$

The filament is represented by the cross sectional area $A$, the perimeter $P$, filament length, the heat capacity $\mathrm{C}$, the density $\rho$ and $\phi$ which represents whether it is the first filament:

$$
\phi=\left\{\begin{array}{c}
1 \text { iffirst filament } \\
0 \text { ifnot first filement }
\end{array}\right.
$$

The environment and the table as well as other filaments are represented by the heat transfer coefficients for convection $\left(h_{\text {conv }}\right)$ and the thermal contact conductance $\left(h_{i}\right)$ for contact $i(i \in\{1,2,3,4,5\})$ (see Figure 35).

The boundary condition $\mathrm{C}_{1}$ can be calculated using the filament temperature $\mathrm{T}_{0}$ at the time to:

$$
C_{1}=T_{0}-Q\left(a_{1}, a_{2}, a_{3}, a_{4}, a_{5}\right)-\frac{h_{c o n v} \phi A T_{E}}{P L b\left(a_{1}, a_{2}, a_{3}, a_{4}, a_{5}\right)}
$$




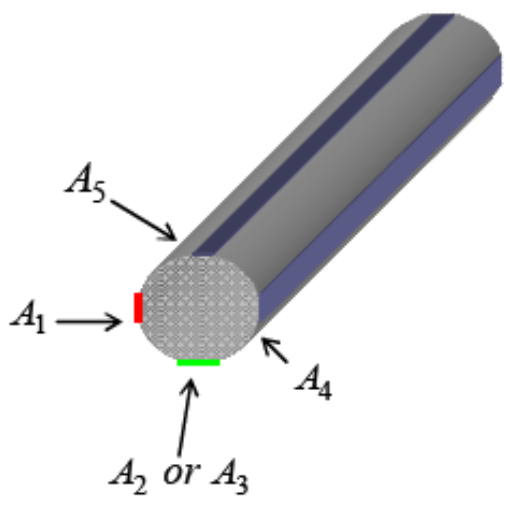

Figure 35 - Filament with possible contact areas $A_{1}, A_{2}, A_{4}, A_{5}$ to filament, $A_{3}$ to table [92]

The functions $Q\left(a_{1}, a_{2}, a_{3}, a_{4}, a_{5}\right)$ and $b\left(a_{1}, a_{2}, a_{3}, a_{4}, a_{5}\right)$ are calculated using the coefficient $a_{i}$ that represent whether or not the filament or table is adjacent (see Figure 35):

$$
\begin{gathered}
a_{i}=\left\{\begin{array}{c}
1 \text { if contact } i \text { exists } \\
0 \text { if contact } i \text { does not exists }
\end{array}, \forall i=\{1,2,3,4,5\}\right. \\
b\left(a_{1}, a_{2}, a_{3}, a_{4}, a_{5}\right)=h_{\text {conv }}\left(1-\sum_{i=1}^{5} a_{i} \lambda_{i}\right)+\sum_{i=1}^{5} a_{i} h_{i} \lambda_{i} \\
Q\left(a_{1}, a_{2}, a_{3}, a_{4}, a_{5}\right)=\frac{h_{\text {conv }}\left(1-\sum_{i=1}^{5} a_{i} \lambda_{i}\right) T_{E}+\sum_{i=1}^{5} a_{i} h_{i} \lambda_{i} T_{i}}{b\left(a_{1}, a_{2}, a_{3}, a_{4}, a_{5}\right)}
\end{gathered}
$$

The fraction of the perimeter $P$ that is in contact with filament or the support is represented by $\lambda_{i}$. The Biot number can be calculated by:

$$
B_{i}=\frac{A}{P} \frac{b\left(a_{1}, a_{2}, a_{3}, a_{4}, a_{5}\right)}{k}
$$

and still has to adhere to $B_{i}<0.1$.

This model assumes that due to the high conductivity the radial and axial heat conduction can be neglected and that contacts between the filaments/laid roads are constant over time which means neglecting gravity and the weight of the filament above. It also transfers the conduction problem between the filament roads to a convection problem (see the use of $h_{i}$ ). Costa et al. [92] showed with 
the simulated results the dependency of cooling on the velocity, filament dimensions, material, sequence of deposition and the environment temperature (see example results in Figure 36).

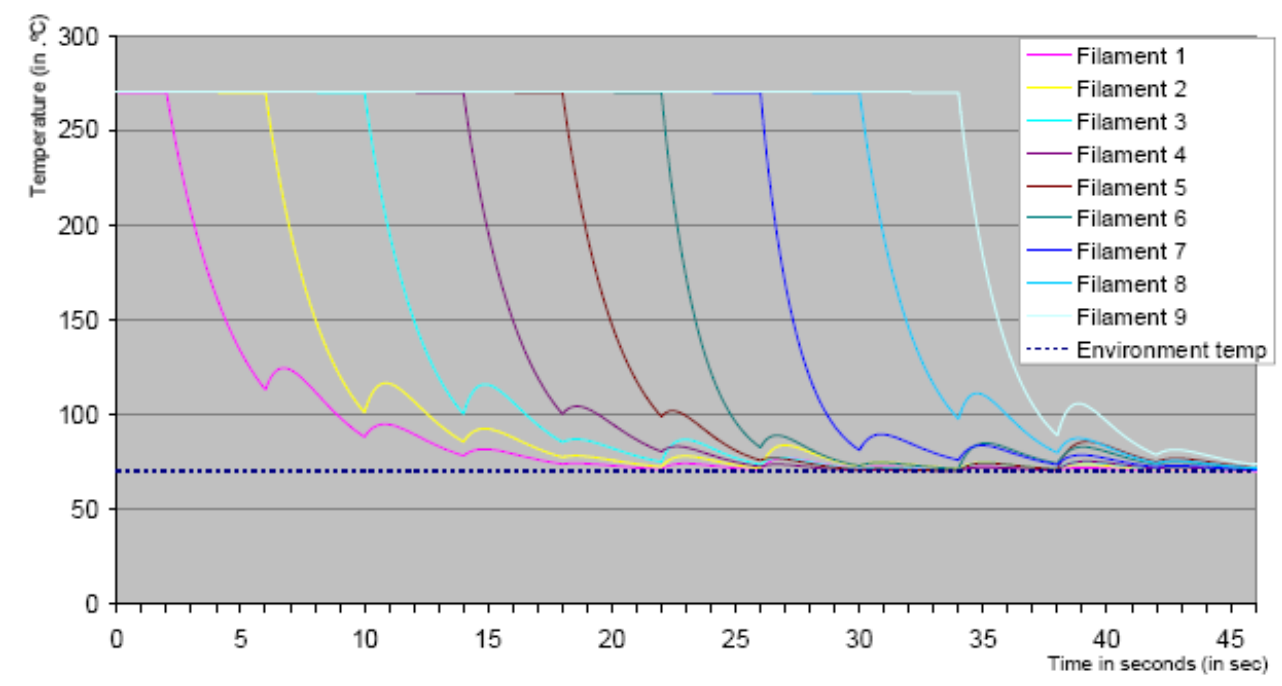

Figure 36 - Simulated time based temperature evolution for 9 filament roads [92]

Finke [71] evaluated the interface temperature suggested by Carslaw et al. [93]:

$$
T_{i}=T_{s s m}\left(1+\frac{\lambda_{d m}}{\lambda_{s s m}} \sqrt{\frac{\alpha_{s s m}}{\alpha_{d m}}}\right)^{-1}+T_{d m}\left(1+\frac{\lambda_{s s m}}{\lambda_{d m}} \sqrt{\frac{\alpha_{d m}}{\alpha_{s s m}}}\right)^{-1}
$$

$\lambda$ represents the thermal conductivity and $\alpha$ the thermal diffusivity of the layer material. Finke used the temperature gradient results (see Figure 37 ) to estimate that the interface temperature $\left(T_{i}\right)$ will be the arithmetic average of the temperature of the deposited semi-solid metal $\left(T_{s s m}\right)$ and the temperature of the previously deposited layer $\left(T_{d m}\right)$.

$$
T_{i} \approx \frac{1}{2}\left(T_{s s m}+T_{d m}\right)
$$




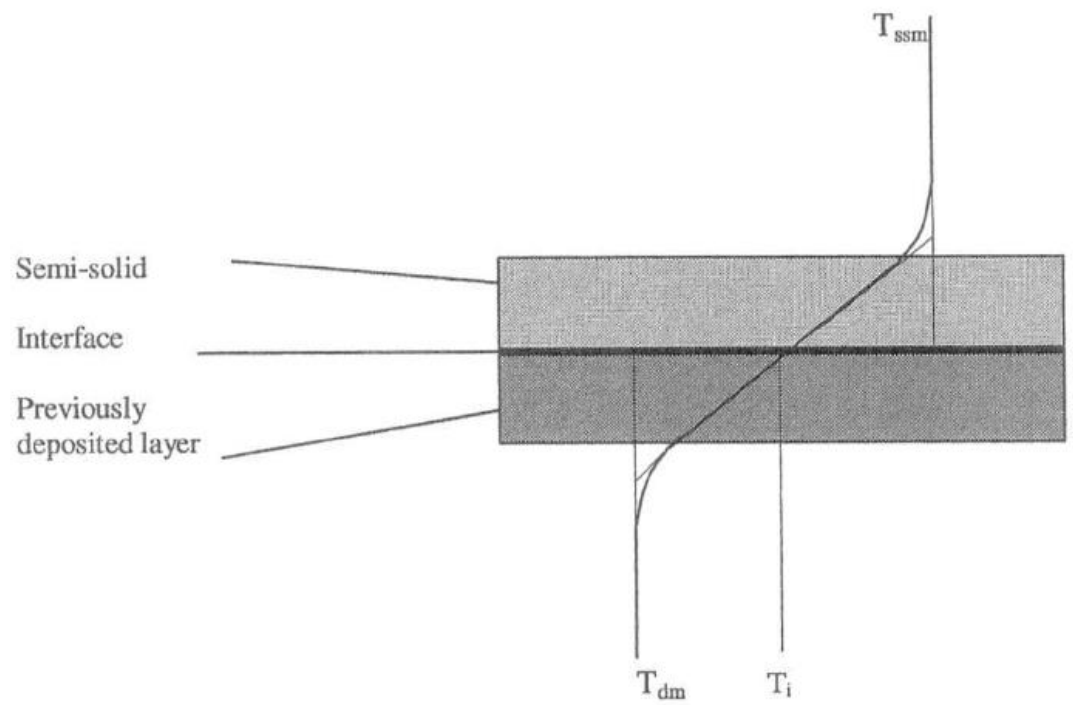

Figure 37 - Temperature gradient and average approximation of layer interface [71]

Besides the review of heat transfer models the growth of the road contact areas also known as neck growth was reviewed by Sun et al. [94]. The neck size expected by the theoretical models is significantly smaller than the measured ones. Sun et al. [94] suggested that the reason for the larger neck growth lies in the creep deformation either from the gravity effect or the downward pressure from the extrusion head during the part building process. It is concluded that this growth will significantly influence the bonding as well as the heat transfer and should be factored in bonding models.

As mentioned before the key control parameter for bonding is the interface temperature. As the temperature of the deposited road is more or less pre-defined through the extrusion the interface temperature can be mainly changed by increasing the temperature of the previously deposited roads. There are several strategies available or possible to increase the temperature of the deposited layer to allow a sufficient residual heat and therefore the interface temperature above a critical temperature e.g. in the semi-solid range:

- Heated build chamber as patented by Stratasys [95]

- Selective heating using forced air [96] 
- Selective heating of metal filament using induction heating coil, similar examples can be found in selective mold heating [97] or using magnetic shielding [98] to direct induced heat

\subsubsection{Conclusion of MEAM}

There are several models proposed to estimate, design or control extrusion and deposition that are applicable for MEAM. When using those all models have to be evaluated for applicability towards semi-solid extrusion and deposition by reviewing the assumptions and simplifications. For instance, adapted analytical bonding heat transfer models can be used to adjust the required selective heating of previously laid roads. But it has to be evaluated whether the boundary conditions allowing the simplification still hold true (e.g. axial/radial heat conduction).

\subsection{Research on MEAM with thixotropic/semi-solid metals}

Based on the growing experience in both areas of thixotropic metal processing and MEAM (mainly through Stratasys Inc. and their FDM) research projects have been conducted in the area of MEAM with thixotropic material. Besides research on MEAM with polymer/metal mixtures ${ }^{16}$ [99-102] two approaches can be identified in the research on MEAM with thixotropic or non-eutectic alloys (see Figure 38).

The first approach was done by Mendez, Rice and Brown at the Massachusetts Institute of Technology (MIT) using a rheocaster to prepare the semi-solid slurry from a molten/liquid bath by cooling and stirring the alloy to the required temperature and microstructure. This resulted in two theses [103, 104], a paper [3] and two patents [105, 106]. These two patents have expired (in 2007/2009) and a founded rheocasting focused company (Semi-Solid Technology Inc.) closed through "involuntary dissolution by Court Order or by the SOC" in 2007 [107].

\footnotetext{
${ }^{16}$ MEAM with polymer/metal filament mixtures are not discussed as this dissertation is focusing on pure metal filaments
} 


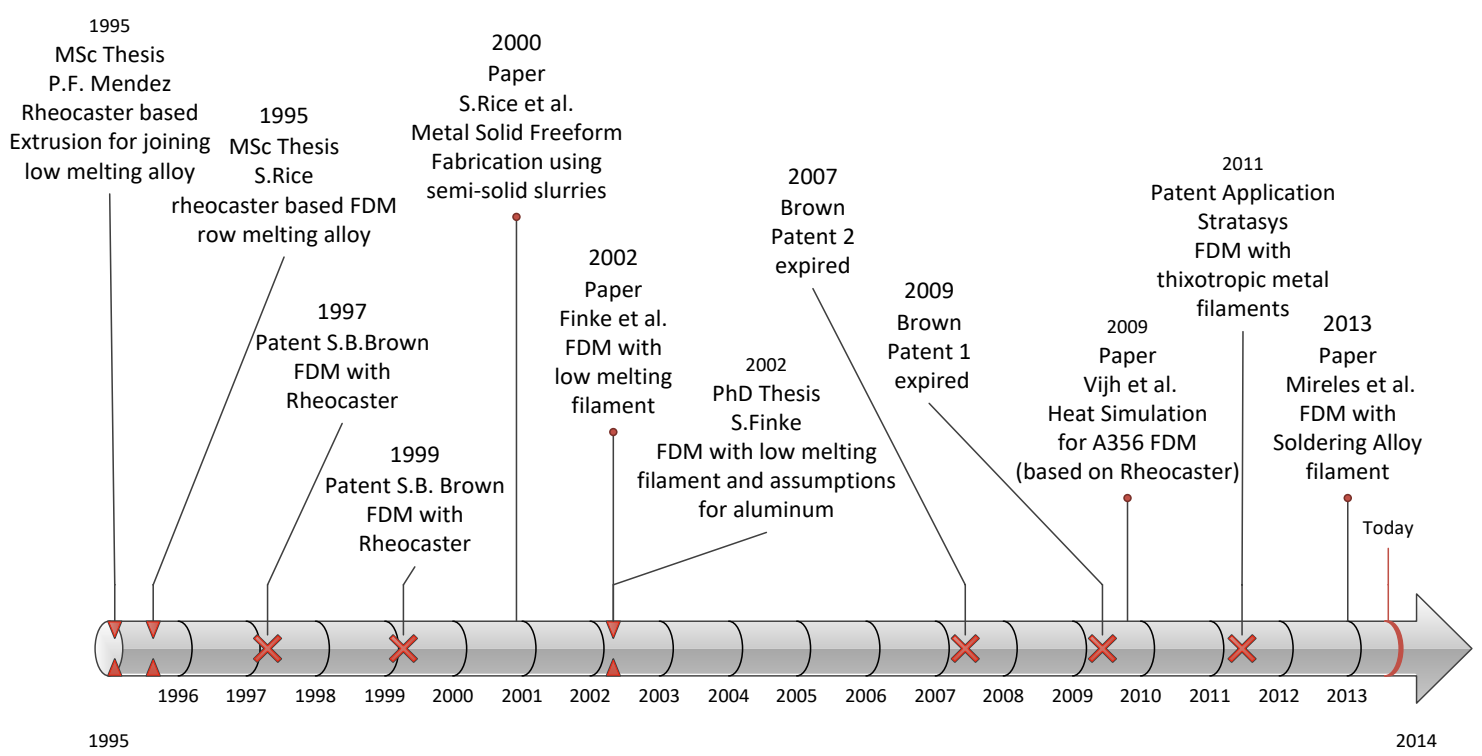

Figure 38 - time bar showing research results and patents for MEAM with thixotropic or non-eutectic metals at the start of this work

Using these rheocaster experiments as a basis Vijh et al. [4] has conducted heat transfer simulations using A356 aluminum alloy properties to identify deposition patterns that would support bonding of layers.

At the Netherlands Organization for Applied Scientific Research (TNO ${ }^{17}$ ) a research project was conducted using low melting alloy filaments reheated to a semi-solid temperature. This resulted in a PhD dissertation [71] and two papers [2, 108].

Stratasys Inc. which owns the first FDM patent [109] as well as a large number of significant ones (e.g. heated build chamber [110]) has filed a patent application for the use of FDM with semi-solid metal filaments [111].

In 2013 experiments were made by Mireles et al. [112] to use low melting temperature solder filaments including a non-eutectic composition (Sn60Bi40) for enabling electronic conduction paths to be added into polymer MEAM objects.

During this dissertation noteworthy literature that has been published are:

- 2017 - Direct metal writing with rheocasting approach [113]

\footnotetext{
${ }^{17}$ Nederlandse Organisatie voor toegepast-natuurwetenschappelijk onderzoek
} 
- 2017 - Fast Desktop-Scale Extrusion Additive Manufacturing [70]

- 2016 - Induction heating of aluminum alloy into semi solid state [114]

Each of these research areas and contributions will be reviewed in detail below.

\subsubsection{Rheocaster approach on MEAM and metal joining}

Rice and Brown studied the rheocaster approach (see Figure 39) for the extrusion of semi-solid metals. Their research included the example extrusion of Sn85Pb15 to either join metal plates together (see Figure 40) or to extrude 3D objects (see Figure 42). As a result of the experiments Rice [104] proposed a dimensionless group (Me) which defines the extruded material in terms of its resistivity of deformation by using the density $(\rho)$, the gravity constant $(\mathrm{g})$, the characteristic time after the viscous flow stops $\left(\mathrm{t}_{\mathrm{f}}\right)$, the apparent viscosity $(\mu)$ and $\mathrm{H}$ as the height of the deposited road:

$$
M e=\frac{\rho g t_{f} H}{\mu}
$$

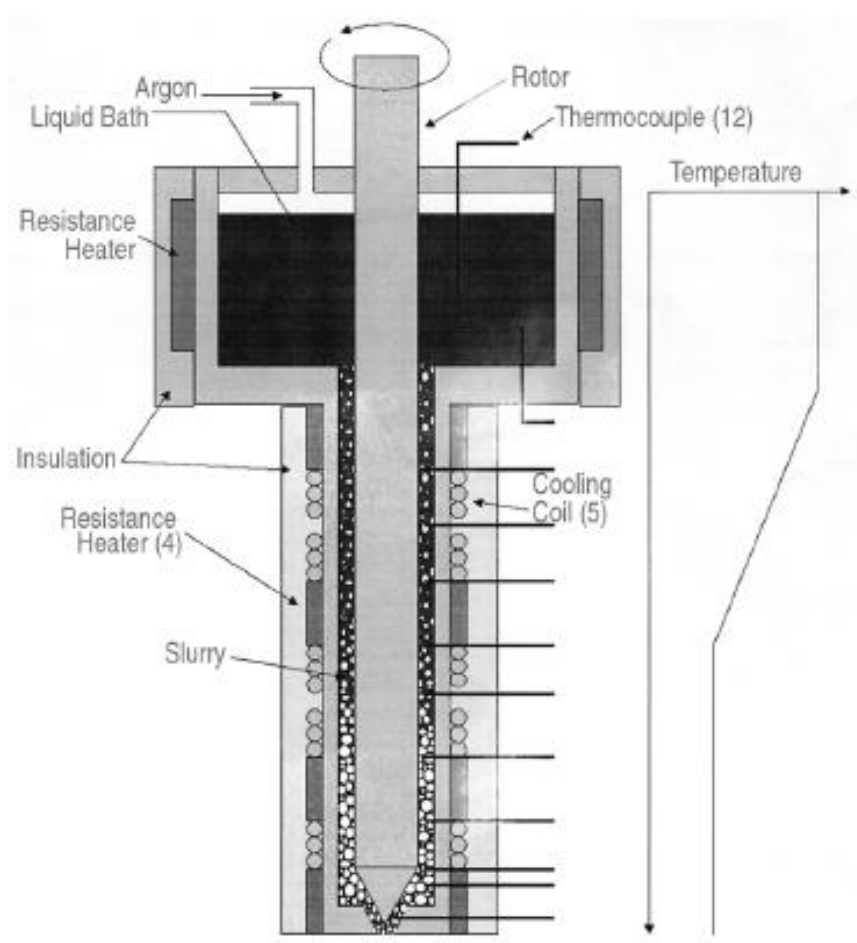

Figure 39 - Rheocaster for extrusion and deposition of semi-solid slurries [3] 
If the group (Me) is much larger than one the deposited road will be liquid like whereas much lower than one will lead to solid-like behavior.

They demonstrated in both projects that the extrusion and deposition of the semisolid metal Sn85Pb15 is possible and will result in sufficient joining of substrates as well as the creation of interface free (see Figure 41) 3D objects. Rice [104] conducted tensile tests with machined specimens from casting and semi-solid deposition. The results showed that the UTS for the deposition process (avg. $53.8 \pm 4 \mathrm{MPa}$ ) were not statistically different than the casting specimens $(55.7 \pm 4 \mathrm{MPa})$.

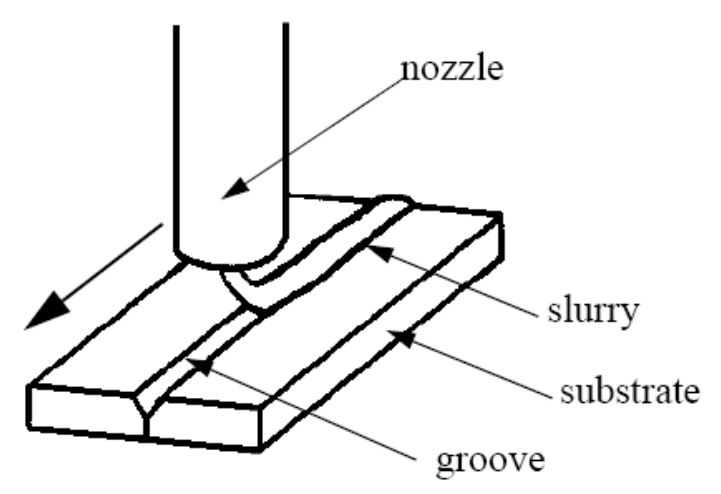

Figure 40 - Semi-Solid joining process [103]

Besides a calculation of the expected shrinkage which is just $2 / 3$ of that of casting, Rice et al. proposed an orifice design that produces square or rectangle roads being extruded to increase the maximum free overhang that can be achieved using the extrusion process. 


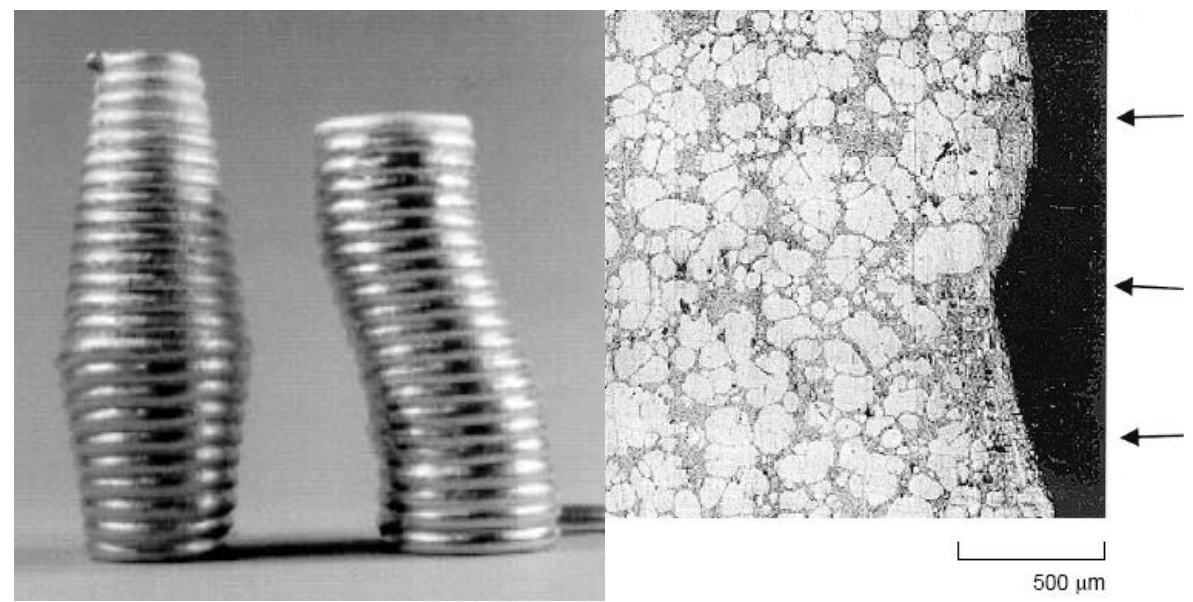

Figure 41 - Sample extruded objects and interface section (middle arrow) [3]

A practical application of the MEAM process with the Rheocaster could not be found and due to the complex apparatus requirements compared to a filament based MEAM approach, it is not likely that this approach would ever be commercially viable.

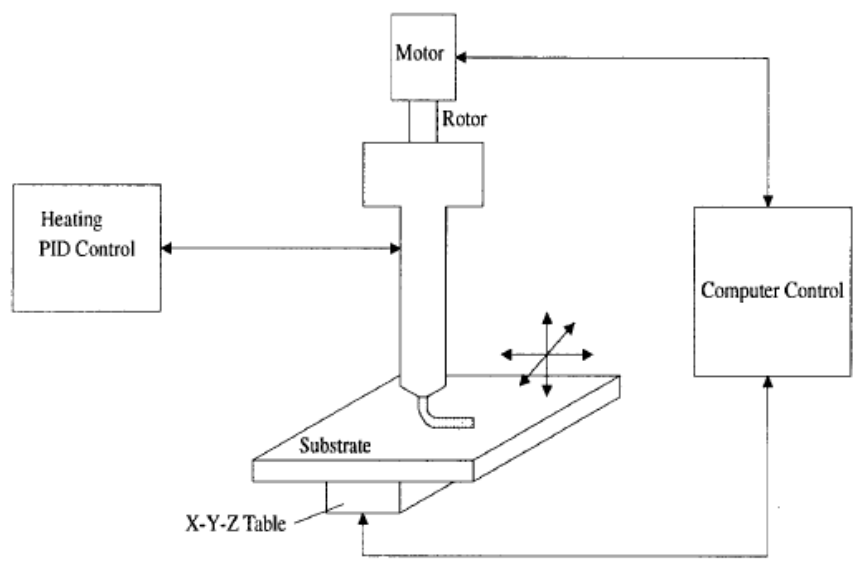

Figure 42 - MEAM setup with rheocaster [104]

\subsubsection{Numeric heat simulation of deposition of aluminum}

Vijh et al. [4] developed a heat transfer model based on the Finite Difference Method to simulate the temperature distribution during deposition of semi-solid metal. The solidification heat is included in the model using the relationship between enthalpy $(H)$ and temperature $(T)$ : 


$$
\begin{gathered}
T(H)= \begin{cases}\frac{H}{C_{p-s o l}}, & H<H_{S} \\
\frac{H-H_{S}}{C_{p-s l u}}, & H \geq H_{S}\end{cases} \\
H(T)= \begin{cases}C_{p-s o l} T, & T<T_{S} \\
H_{S}+C_{p-s o l}\left(T-T_{\text {slu }}\right), & T \geq T_{S}\end{cases}
\end{gathered}
$$

The enthalpy at a temperature (T) is represented by $H(T)$, the enthalpy at the solidus temperature by $H_{S}$, the solidus temperature is represented by $T s$, the constant slurry temperature by $\mathrm{T}_{\mathrm{slu}}$, the specific heat capacity of the solid by $\mathrm{C}_{\mathrm{p} \text {-sol }}$ and of the semi-solid by $\mathrm{C}_{\mathrm{p} \text {-slu. }}$. As a simulation example material Vijh et al. utilized A356 aluminum alloy parameters. The following assumptions to simplify the model are made:

- Fixed square slurry cross section with no deformation after deposition

- No temperature and solid fraction change during deposition itself

- No gaps in the deposited material / full direct connections of roads

- Temperature gradient within the slurry/road cross section is neglected

- Repositioning of the rheocaster/base plate takes no time - no cooling

The simulation was executed to review the maximum height that could be deposited with fully fused layers ${ }^{18}$ compared to the deposition rate (see Figure 44 ) as well as the best deposition pattern comparing rotational, unidirectional and alternating patterns (see Figure 43 - Simulated deposition pattern [4]Figure 43).

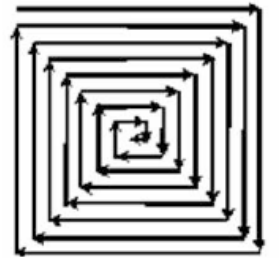

(a)

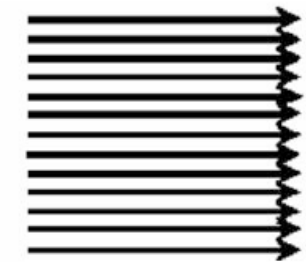

(b)

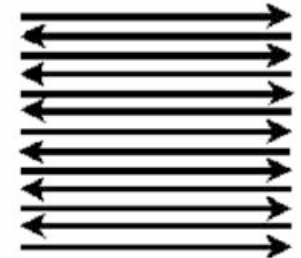

(c)

Figure 43 - Simulated deposition pattern [4]

\footnotetext{
${ }^{18}$ Assumption was used that the interface need fall within the semi-solid rage for fully fused layers
} 
Vijh et al. found that the alternating pattern is the most optimal one using the assumptions. The unidirectional and alternating showed a similar optimal results but it had to take into account that the repositioning takes additional time so the slurry would be able to cool.

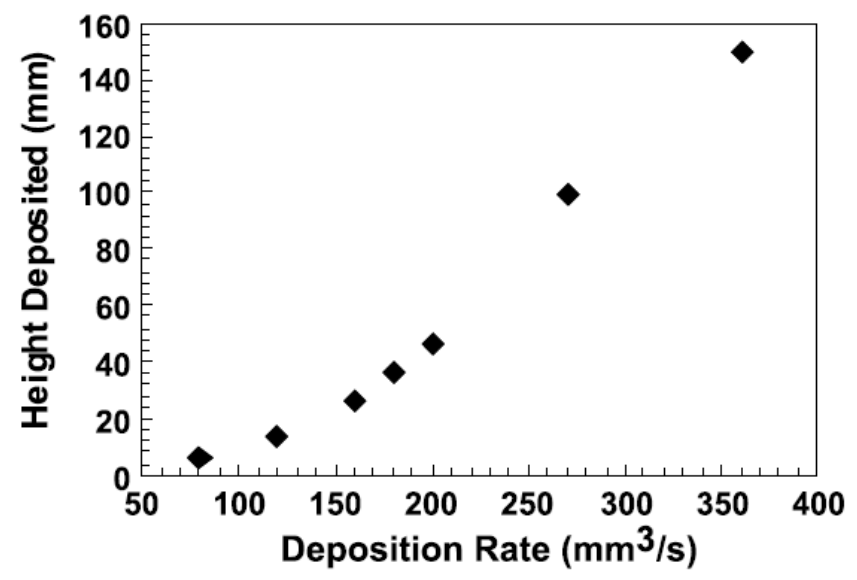

Figure 44 - fully dense deposition height based on the extrusion rate [4]

One further assumption Vijh took was that the base plate is heated to the semisolid slurry temperature used. When using a closed build environment that may not be practical due to the heating of the full apparatus.

\subsubsection{Solid Freeform Fabrication of metal components by extrusion and deposition of semi-solid metals}

Besides the Stratasys Inc. patent application the work conducted by Finke et al. [2, $71,108]$ is the most applicable work towards this dissertation project. Finke in her dissertation [71] reviewed $\mathrm{Pb}-\mathrm{Sn}, \mathrm{Sn}-\mathrm{Bi}, \mathrm{Sn}-\mathrm{Pb}-\mathrm{Bi}$ and $\mathrm{AL}-\mathrm{Ge}$ alloys for their applicability for extrusion and deposition of semi-solid metals (EDSSM). As part of that review solidification experiments $(70 \mathrm{Sn} .30 \mathrm{Bi})$ and Microstructure analysis $(60 \mathrm{~Pb} 20 \mathrm{Bi} \& \mathrm{Al}-17 \mathrm{Ge})$ were performed. Important extensions of the $\mathrm{AlGe}$ experiments were a review of the influence of cold rolling to the globular particle size. Finke concluded similar to what was found in the literature before (see also chapter 2.2.3) that cold rolling reduces the size of the globular particle. Also a cold reduction above $20 \%$ did not have any further reduction effects as well as the effect itself will be visible over time increase for longer isothermal holding times $15-30 \mathrm{~min}$. 
Using Sn70Bi30, rheological studies were performed with three types of experiments: a capillary viscometer, plate-plate rheometer and a back-extrusion experiment (see Figure 45).
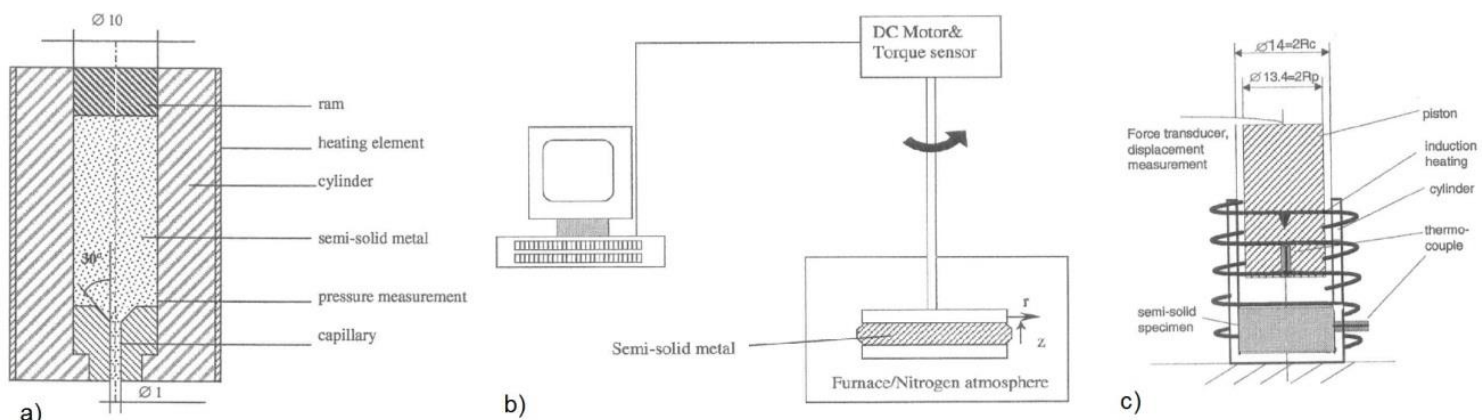

Figure 45 - Rheological studies of Finke [71] a) capillary viscometer, b) plate-plate rheometer, c) back extrusion experiment

Only the back-extrusion experiment produced meaningful results to evaluate the filament material towards its appropriateness for EDSSM. During back extrusion a power law index of $n=0.8$ was determined. Also significant segregation could be identified in the capillary and back extrusion experiment. When reviewing the results Finke concluded that the segregation effects can be observed when the force is below a critical value at a certain velocity. When comparing the force required for squeezing out the liquid and the normal force required for extrusion, those force functions meet at a critical velocity (see Figure 46) e.g. $8-9^{*} 10^{-3} \mathrm{~m} / \mathrm{s}$ for $\mathrm{Pb} 60 \mathrm{Sn} 40$ at $0.4 \mathrm{fs}$. 


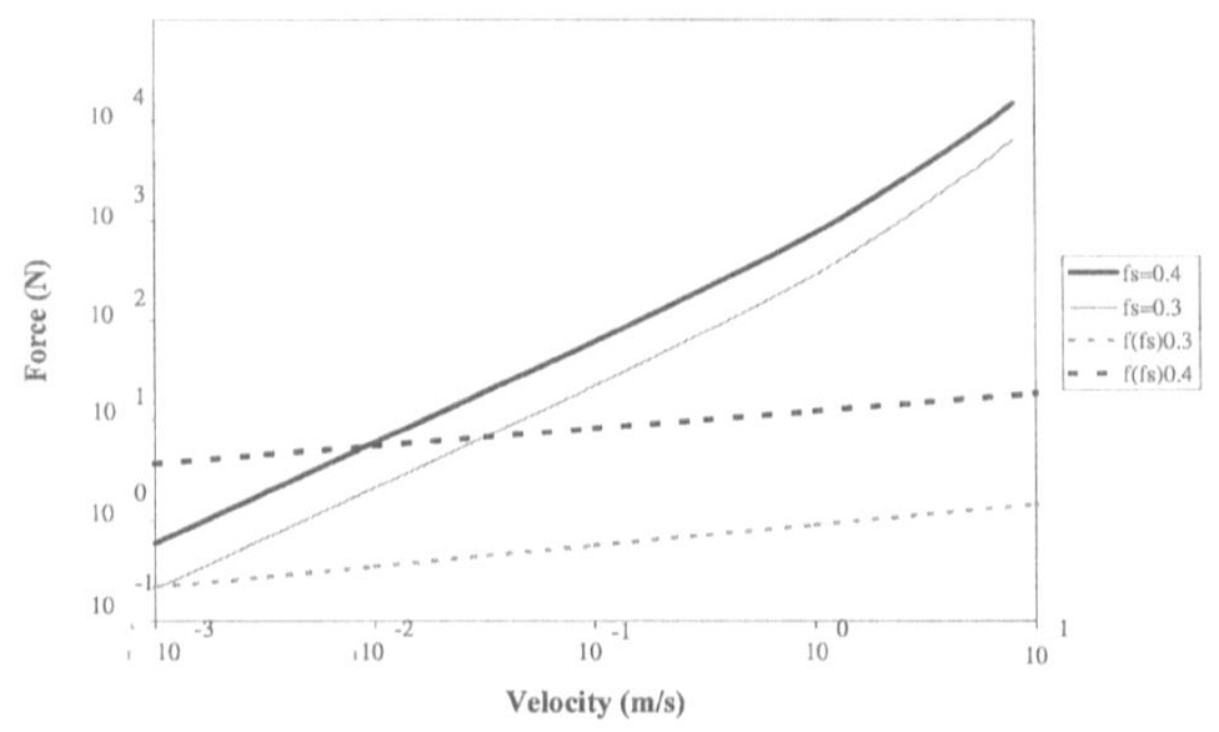

Figure 46 - Force resulting in segregation (solid) vs. extrusion force (dotted) [71]

This led to a start-stop phenomenon where higher liquid content in the semi-solid was extruded during the start and after reducing velocity in some cases clogging was observed as well as delayed stopping and liquid droplets.

Finke measured the extrusion force via a load cell integrated in the experimental nozzle (see Figure 47) and compared this with the calculated extrusion forces via existing models. Measurements have been observed with 3-times higher force values but a model that would fit the real values has not been found. Finke concluded that a model for the required force could be used for a more direct control of the segregation via changes of applied force when it drops below the critical value. 


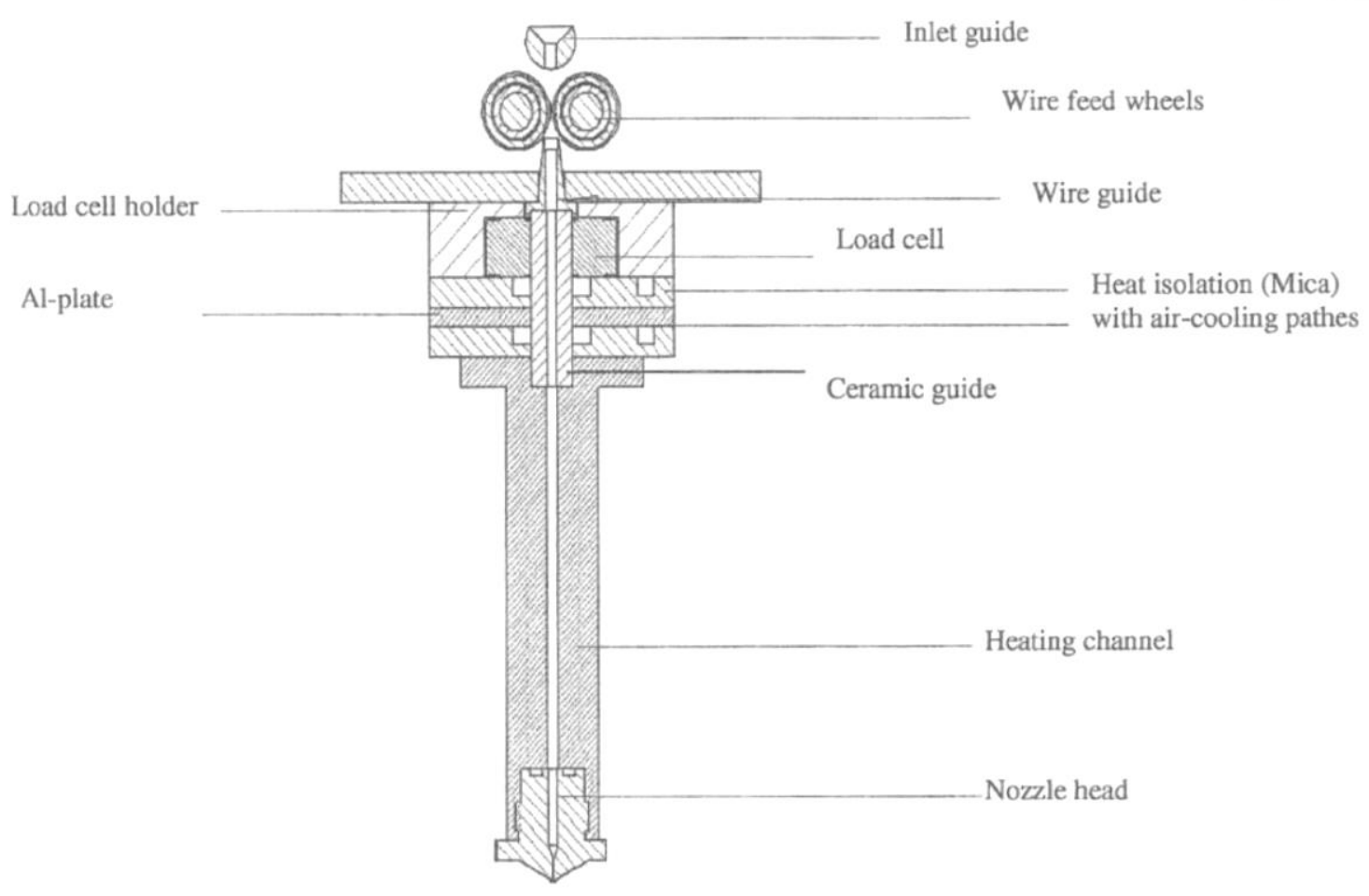

Figure 47 - Experimental nozzle including force measurement via load cell [71]

Finke reviewed the bonding requirements and concluded on the basic requirement from Rice et.al.[3] that the interface temperature must fall in the semi-solid range and that one possibility is a heated build environment just below the semi-solid range (e.g. $160^{\circ} \mathrm{C}$ for a filament temperature of $210^{\circ} \mathrm{C}$ resulting in an interface temperature of about $185^{\circ} \mathrm{C}$ ).

The extrusion rate compared to the $x-y$ velocity was determined to be at a rate of 1:1. When reviewing the extrusion itself Finke concluded that shear thinning through the extrusion could not be observed at an extrusion ratio of 25 .

Finke overall found the following problems that need to be reviewed:

- Segregation and clogging at a low filament velocity or force

- High environment temperature leads to lower mechanical properties of the extruded part

- Coarsening of the microstructure during holding times 
- Capillary and plate-plate rheometer are not adequate to review such semisolid alloys

- Force measurement and control should be applied and a model needs to be developed to differentiate between friction, extrusion and capillary force to detect and compensate for inhomogeneous flow

As one of the key results Finke developed a process window for EDSSM that allows an application to build 3 dimensional parts using Pb40Sn60 (see Figure 48) and demonstrated that this window is sufficiently large to operate in.

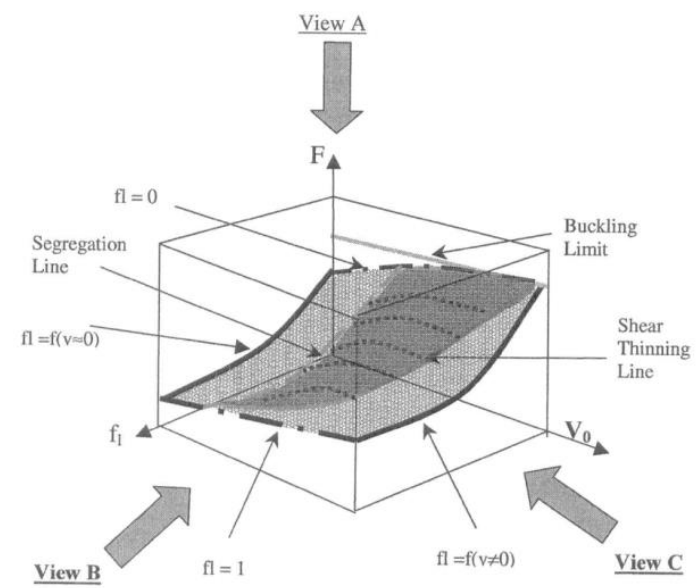

a)

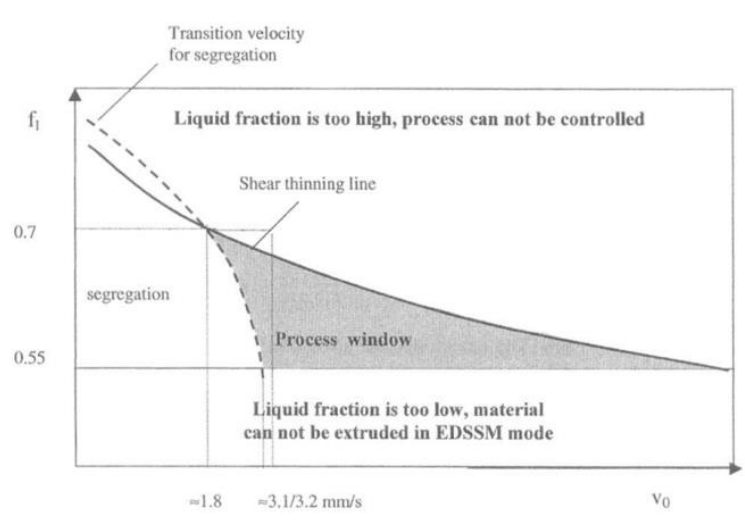

b)

Figure 48 - Process window for EDSSM a) 3D quantitative representation, b) cross section through the process volume

Finke concluded her work by discussing the application of her results to an aluminum alloy filament and suggested the following design elements:

- Grain refinement should be used to allow minimal grain size / small nozzle orifice while assuming that the grains should not be larger than $1 / 6$ of the nozzle orifice.

- Induction heating should be used compared to resistive heating which also is assumed to reduce the start stop phenomena. A heating with a power of $2 \mathrm{KW}$ and a frequency of $500 \mathrm{kHz}$ to $1.2 \mathrm{MHz}$ was tested and deemed adequate. 
- To reach the interface temperature, (sub) build environment heating, selective laser heating and selective induction heating was discussed where the preference was selective inductive heating.

- To stabilize the particle size or also allow larger grains without clogging, nozzle agitation was suggested e.g. via piezo agitation.

\subsubsection{Stratasys Patent for FDM with metal}

Stratasys Inc. has filed a patent application[111] for a system that uses semi-solid metal to create layer based 3D objects. This basically describes their FDM apparatus for use with semi-solid metals. This patent application includes a friction wheel or screw pump filament transport mechanism, a heated build platform above $350^{\circ} \mathrm{C}$ and an oxygen free build chamber. The patent application also explains the expected creation of non-dendritic microstructures to allow a semi-solid deposition at a sufficient viscosity.

Worthy to note is that the example extrusion of the AISi alloy was at $610^{\circ} \mathrm{C}$ and if this was done using A356 or a similar alloy with such a silicon portion the temperature range would be in a very sensitive range in terms of changes of viscosity (see section 2.1.8). Also, as the example microstructures shown in the figures are only from heating experiments and no final extruded 3D objects are displayed, it is assumed that Stratasys does not yet have the full process under control.

\subsubsection{Use of low melting temperature soldering alloys}

Mireles et al. [112, 115] refitted a Stratasys FDM 3000 system with a straight liquefier (see Figure 49) to reduce the pressure drop and therefore the extrusion force (see also section 2.3.2.3) to support the extrusion of two low melting temperature soldering alloys (eutectic Bi58Sn42 and non-eutectic Sn60Bi40). The

extrusion of both alloy types is done above the liquidus temperature and not in the semi-solid range of the non-eutectic alloy. Still Mireles et al. could create multilayer 3D objects using this soldering alloy (see Figure 50). 


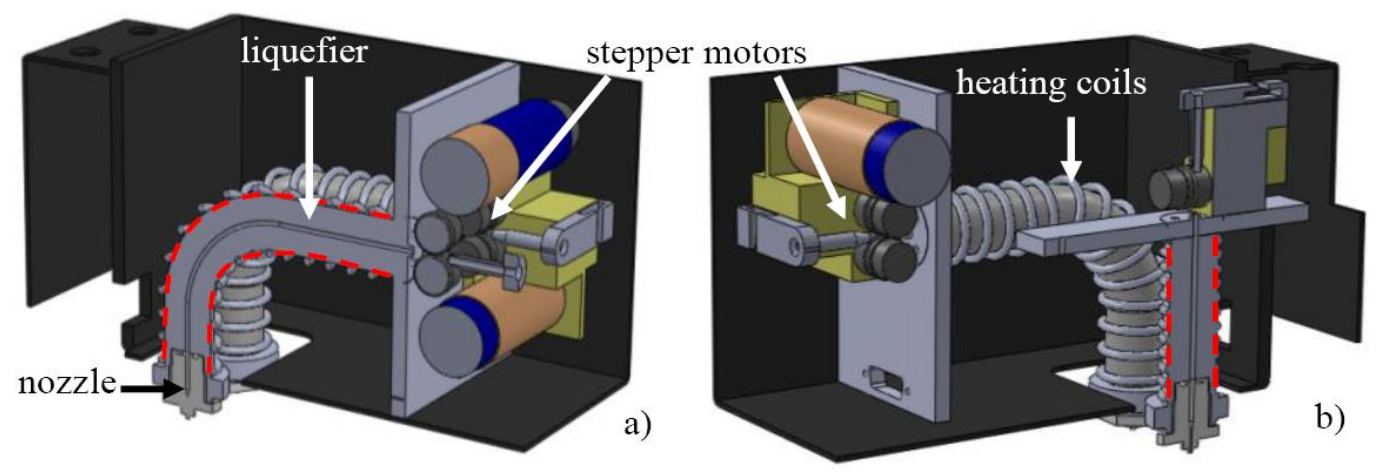

Figure 49 - a) standard curved liquefier of FDM 3000 b) added a strait liquefier [115]

Mireles also demonstrated extrusion of a metal alloy combined with the extrusion of ABS filament. This was tested to prove the combination of the two materials to for example allow embedded electronics.
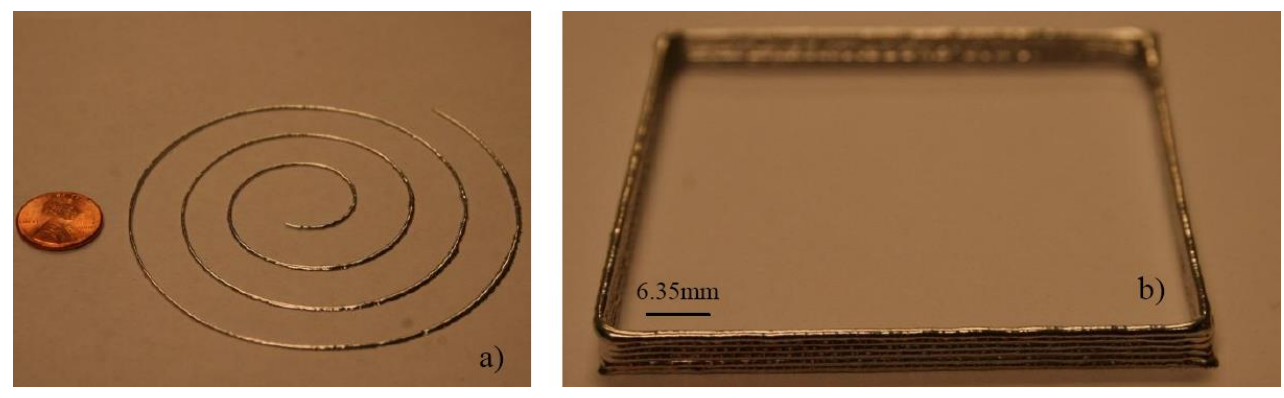

Figure 50 - Extrusion examples for a) 2D and b) 6 layer 3D structures [115]

Mireles et al. concluded that further research on higher melting metals and better temperature control resulting in microstructure and interfacial bonding control is required.

\subsubsection{Direct metal writing with rheocasting approach}

Chen at al. [113] performed further (see also section 2.4.1) experiments with a rheocaster approach. The work was published as Direct Metal Writing DMW using $\mathrm{Bi}_{75} \mathrm{SN}_{25}$ instead of $\mathrm{SN}_{85} \mathrm{~Pb}_{15}$ by Rice et al. [3]. The Rheocaster was designed using multiple resistive heating circuits with thermocouple feedback (see Figure 51) resulting in a precision of $\pm 2^{\circ} \mathrm{C}$. During the experiments a nozzle orifice diameter of $1 \mathrm{~mm}$ with a substrate distance of $0.7 \mathrm{~mm}$ was used. 

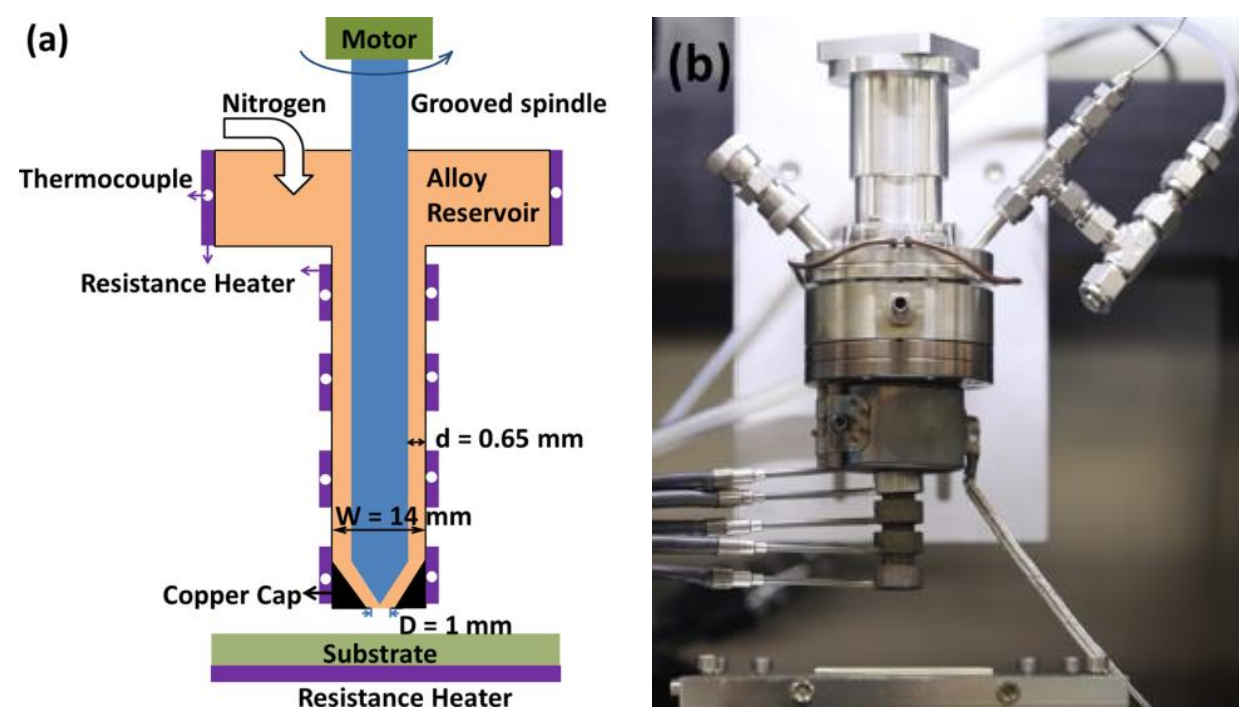

Figure 51 - Direct Metal Writing - a) Rheocaster design b) experiment setup [113]

The extruded material was layered on a substrate and similar to Rice et.al [3] reviewed were performed whether adequate bonding can be achieved. A critical influence in the observed bonding was pre-heating of the substrate material (see Figure 52).
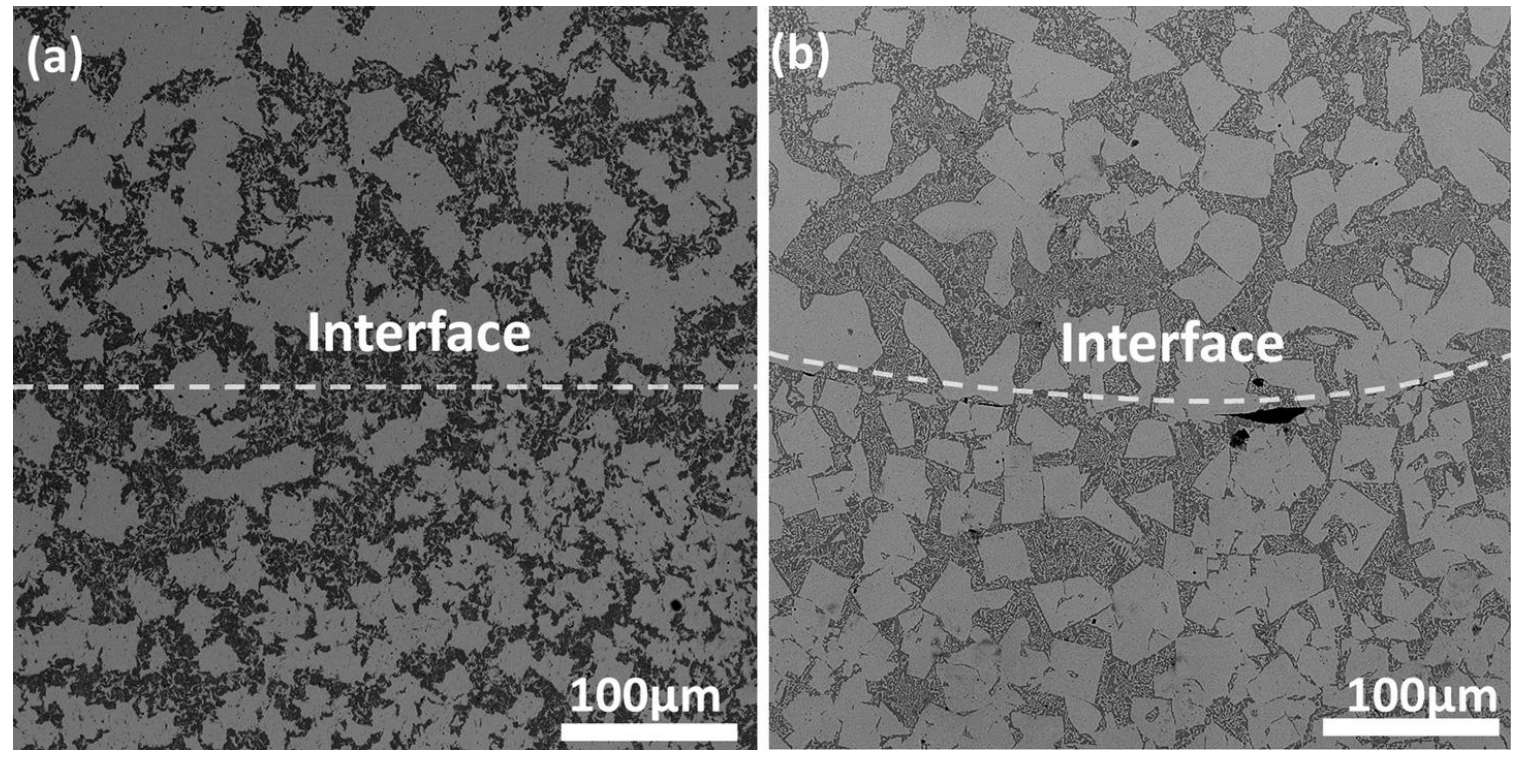

Figure 52 - Rheocasting bonding a) adequate bonding with pre-heated substrate b) Inadequate bonding without pre-heating of substrate [113]

This does correlate to the results of the work of Finke [71] and Carslaw et al. [93] (see Figure 37). 


\subsubsection{Fast Desktop-Scale Extrusion Additive Manufacturing}

While not direct related to MEAM Go et.al developed, besides a nut based filament feeding, a double heating system for the FastFFF [70]. This includes a laser-based pre-heating and a resistive based liquefier heating (see Figure 53).

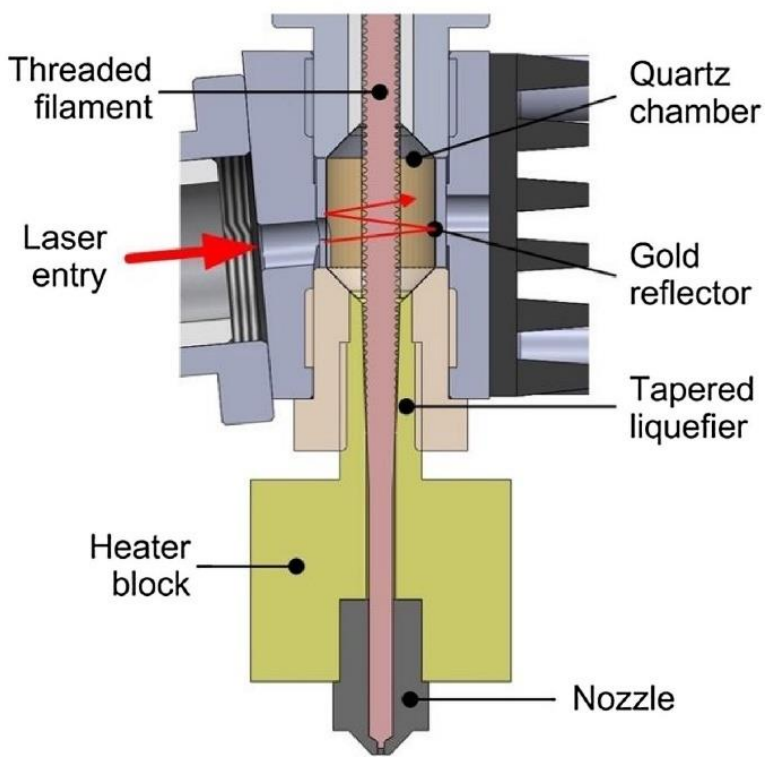

Figure 53 - FastFFF Extruder with laser pre-heating [70]

This process does allow a faster extrusion reaching up to $177 \mathrm{~cm} 3 / \mathrm{h}$ for $1 \mathrm{~mm}$ nozzle orifice. A dual heating process with preheating the filament and then resistive heating to achieve a semi-solid state might be an applicable approach for MEAM as well. Pre-heating might be performed by laser or induction heating as both would allow a fast start up and stopping.

\subsubsection{Induction heating of aluminum alloy into semi solid state}

As part of a master thesis [114] Garcia developed an induction heating process for Aluminum-Fused-Filament Fabrication (AI-FFF) using AA6061 (see Table 6 for composition).

Table 6 - Composition of AA6061 [114]

\begin{tabular}{|c|c|c|c|c|c|c|c|c|}
\hline Si & Fe & Cu & Mn & Mg & Zn & Ti & Cr & Al \\
\hline
\end{tabular}




\begin{tabular}{|c|c|c|c|c|c|c|c|c|}
\hline $0.4-$ & 0.7 & $0.15-$ & 0.15 & $0.8-$ & 0.25 & 0.15 & $0.04-$ & Balance \\
0.8 & $\max$ & 0.40 & $\max$ & 1.2 & $\max$ & $\max$ & 0.35 & \\
\hline
\end{tabular}

For induction heating purposes of AA6061 Garcia developed a feeding enclosure allowing the oxygen layer free filament processed into the Nozzle (see Figure 54). The aluminum filament $(3.18 \mathrm{~mm})$ was manually processed using an abrasive grit pad to remove the natural aluminum-oxide layer and quickly placed into the oxygen free feeding enclosure.

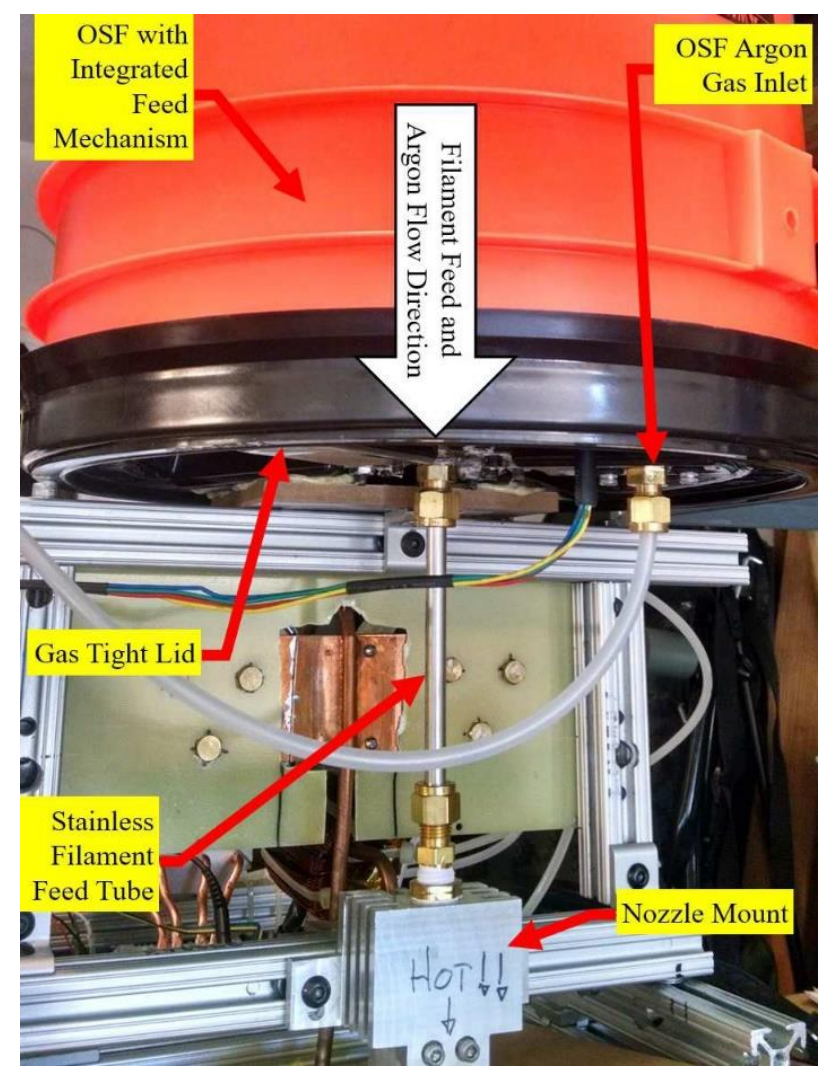

Figure 54 - Oxygen shielded feeder for AI-FFF [114]

The initial aluminum oxide ceramic $\left(\mathrm{Al}_{2} \mathrm{O}_{3}\right)$ nozzle was replaced with a boron nitride ceramic (BNC) nozzle due to fusion with the semi-solid aluminum alloy. Also the BNC nozzle broke twice during the experiments as such Garcia proposed future research needed to build a nozzle that can withstand the high temperature as well as the lateral and compression stress. During the experiments the work coil observed incidental induction energy losses due to other system components or 
tooling nearby. This effect has been noted as a potential barrier for an Al-FFF system. For example, a distance lower then $5 \mathrm{~mm}$ between the work coil and the bed the amperage raised significantly due to the induced current in the bed. A spike based (Mesa baseplate) bed was created to minimize the interference with the work coil.

Ambient air experiments showed the oxide layer prevented the extrusion process and later experiments showed significant issues with the temperature control of the induction heater for the narrow semi-solid window. Planned fusion of extruded material with the base plate was not possible due to the large temperature difference and only extruded material with overheated material was able to fuse. Garcia proposed an induction coil at the nozzle tip to head the extruded layer and allow fusion with the new layer.

The work resulted in a provisional patent 62300188 (not available for public review).

\subsubsection{Conclusion of MEAM with thixotropic/semi-solid alloys}

It can be seen from the literature that efforts have been made to extrude semi-solid alloys to build (or integrate in) 3D objects. None of the literature could fully verify the application of semi-solid aluminum alloys for the MEAM process. But it could be seen where the challenges could be expected:

- Controlling the microstructure before or during the extrusion and deposition

- Heating of the filament and previously deposited layer

- Managing start-stop phenomena including segregation of liquid and solid state

- Managing the existing oxidation layer of the filament as its liquid temperature is above $2000^{\circ} \mathrm{C}$

- Managing the oxygen affinity of the deposited layer 


\section{INITIAL EXPERIMENTS}

\subsection{Initial filament review}

Using a 2mm diameter Al4018 filament from Drahtwerke Elisental [116] initial heating experiments at the Foundry Institute - RHTW Aachen [117] were conducted. This specific filament was chosen as Al4018 appeared to be insignificantly different from the A356 standard for the purposes of this research, as it had slightly increased magnesium and decreased copper percentage (see Table 7) and the filament was commercially available:

Table 7 - Alloy composition compare Al4018 vs. A356

\begin{tabular}{|l|c|c|c|c|c|c|c|c|}
\hline & $\mathrm{Si}$ & $\mathrm{Fe}$ & $\mathrm{Cu}$ & $\mathrm{Mn}$ & $\mathrm{Mg}$ & $\mathrm{Zn}$ & $\mathrm{Ti}$ & $\mathrm{Al}$ \\
\hline Al4018${ }^{19}$ & $6.5-$ & 0.2 & $\mathbf{0 . 0 5}$ & 0.1 & $\mathbf{0 . 5 - 0 . 8}$ & 0.2 & 0.2 & remaining \\
& 7.5 & & & & & & & \\
\hline A356 & $6.5-$ & 0.2 & $\mathbf{0 . 2}$ & 0.1 & $\mathbf{0 . 2 5 -}$ & 0.2 & 0.2 & remaining \\
& 7.5 & & & & $\mathbf{0 . 4 5}$ & & & \\
\hline
\end{tabular}

The filament is produced in a typical drawing process at the manufacturer via the following steps after initial extrusion to a $9.6 \mathrm{~mm}$ raw wire:

1. Drawing process from $9.6 \mathrm{~mm}$ to $5.8 \mathrm{~mm}$

2. Isothermal holding at $380^{\circ} \mathrm{C}$ for 10 hours

3. Drawing process from $5.8 \mathrm{~mm}$ to $3.8 \mathrm{~mm}$

4. Drawing process from $3.8 \mathrm{~mm}$ to $2.0 \mathrm{~mm}$

As mentioned in section 2.2.4 the drawing process is similar to the SIMA process and during reheating a globular structure can be expected (melt activation).

The as-is filament shows a typical severe deformation microstructure where a grain size cannot be determined (see Figure 55).

\footnotetext{
19 DIN EN 573-3
} 


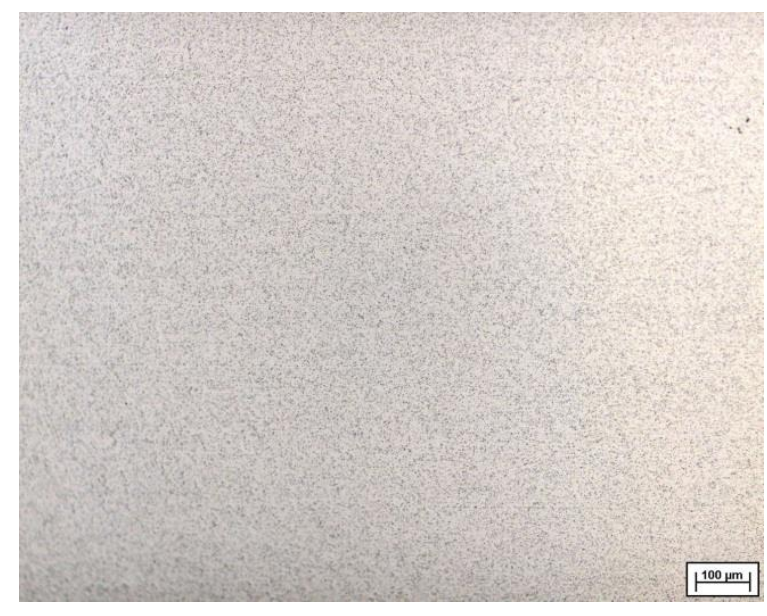

Figure 55 - as-is filament Al 4018

From conversations with the filament vendor the last step of the filament production process was performed as a cold operation and no heat treatment was applied after that. It is expected that this results in an energy increase in the filament due to an increase of dislocations (see section 2.1.1). Therefore, the filament should re-crystalize during heating and isothermal holding at a semi-solid temperature range with a fine globular grain size. This can be compared to the SIMA process route explained in chapter 2.2.3.

A first experiment of heating the filament was conducted and the expected globular grain structure was found at an isothermal holding time of 15 minutes (see Figure 58). Further experiments have been conducted to quantify the microstructure through the fraction solid, form factor and globular diameter. Al4018 with its AISi7 alloy components should show an approximate $50 \%$ fraction solid at $585^{\circ} \mathrm{C}$. As such the factors for the temperature have been defined as $575^{\circ} \mathrm{C}, 580^{\circ} \mathrm{C}, 585^{\circ} \mathrm{C}$ and $590^{\circ} \mathrm{C}$. The heating time to the target temperature of $585^{\circ} \mathrm{C}$ was determined using a type $\mathrm{K}(\mathrm{Ni}-\mathrm{CrNi})$ thermocouple attached to the filament when heating in the air-flow oven ${ }^{20}$ (see Figure 56).

${ }^{20}$ Brandhorst air-flow oven including a Nabertherm C40 temperature control unit 
The filament was placed in the air-flow oven on a bed of quarry sand. Within the first approximately 60 seconds the filament heated up to about $583^{\circ} \mathrm{C}$ while to reach the oven temperature of $585^{\circ} \mathrm{C}$ it took overall 120 seconds.

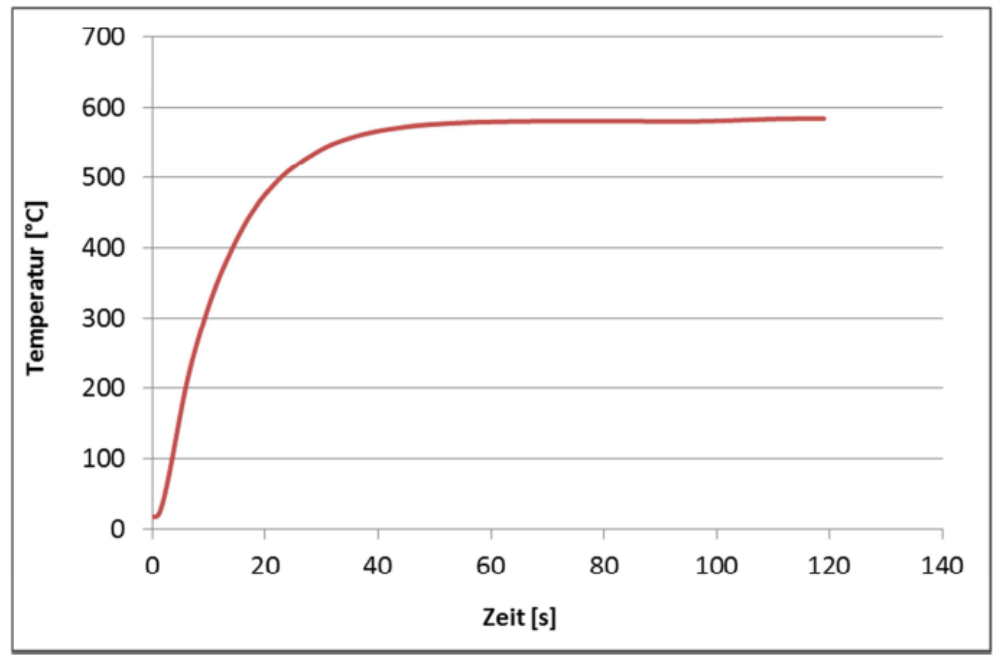

Figure 56 - Time required for the filament to reach $585^{\circ} \mathrm{C}$ in the air-flow oven

After holding for $15 \mathrm{~min}$ at the defined temperature the filament was then quenched in a water bath at room temperature. This process was repeated for the other temperature factors and micrographs were taken (see Figure 58). The micrographs were then analyzed using automatic separation ${ }^{21}$ of grains (see Figure 57 for an example picture of automatically separated grains) and measurement of form factor and diameter of the grain assuming a perfect cycle with the same area.

${ }^{21}$ Using Carl Zeiss KS400 V3.0 


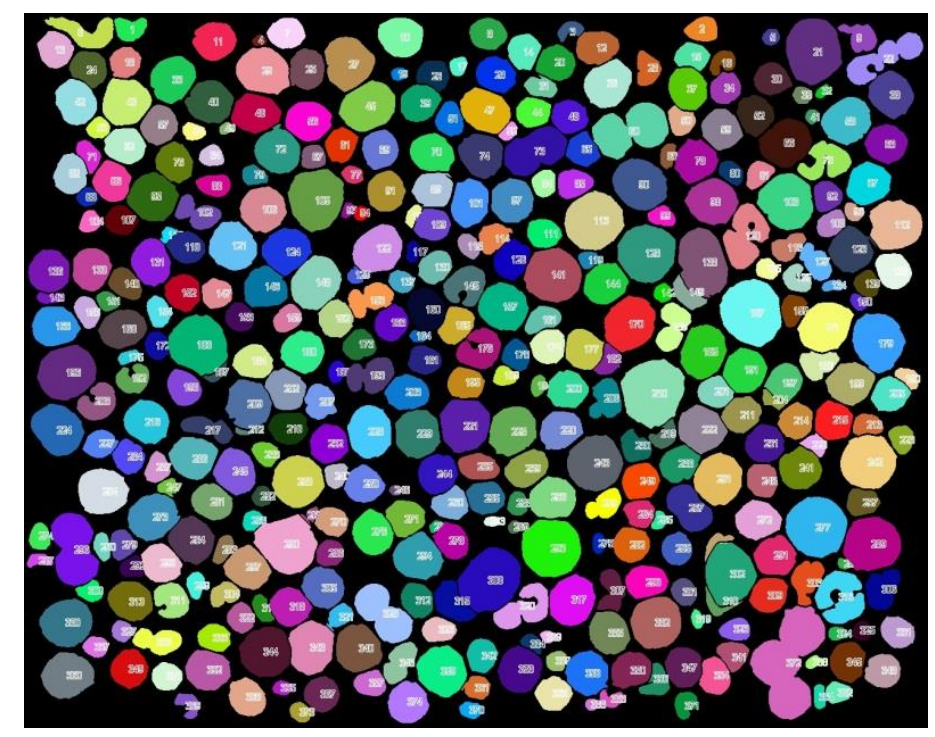

Figure 57 - Picture of micrograph example $585^{\circ} \mathrm{C}$ with automatic grain separation and numbering Using these values the fraction solid has been calculated. Each factor was repeated 4 times.

The results (see Table 8 and Figure 59) show a small average grain size between $44.6 \mu \mathrm{m}$ and $49.4 \mu \mathrm{m}$ which is sufficiently small for semi-solid extrusion and a good form factor above 0.87 . Comparing the temperature $575^{\circ} \mathrm{C}$ and $590^{\circ} \mathrm{C}$ coarsening can be observed (see Figure 58).

Table 8 - filament isothermal holding for $15 \mathrm{~min}$ at different temperatures results

\begin{tabular}{|c|c|c|c|c|}
\hline $\mathbf{T}\left[{ }^{\circ} \mathbf{C}\right]$ & $\mathbf{T}[\mathbf{m i n}]$ & $\mathbf{f s}_{\mathbf{s}}[\mathbf{\%}]$ & $\mathbf{F}$ & $\mathbf{D}$ circle $[\boldsymbol{\mu m}]$ \\
\hline 575 & 15 & 69.6 & 0.87 & 44.6 \\
\hline 580 & 15 & 67.7 & 0.89 & 48.1 \\
\hline 585 & 15 & 66.8 & 0.88 & 48.5 \\
\hline 590 & 15 & 64.5 & 0.88 & 49.4 \\
\hline
\end{tabular}




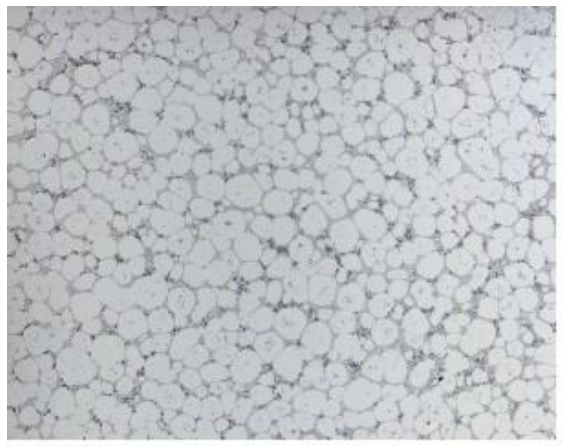

$575^{\circ} \mathrm{C}$

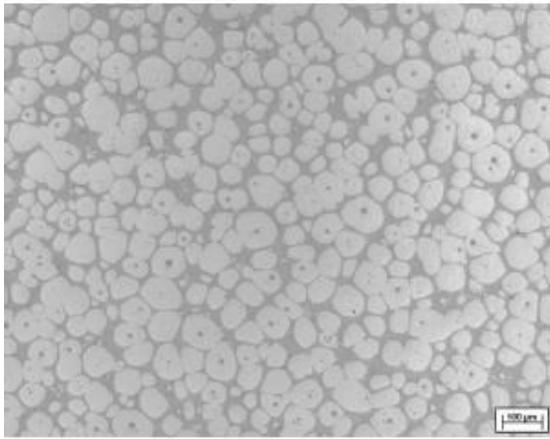

$585^{\circ} \mathrm{C}$

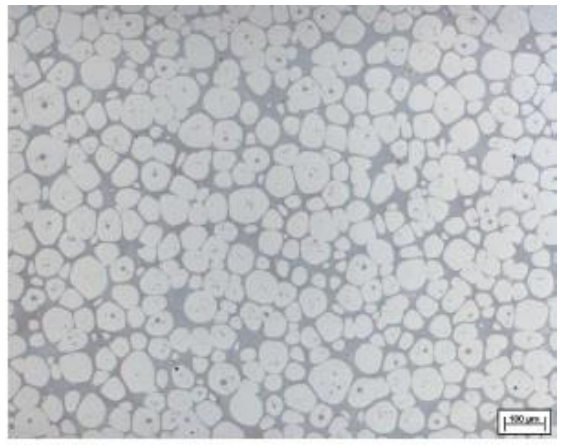

$580^{\circ} \mathrm{C}$

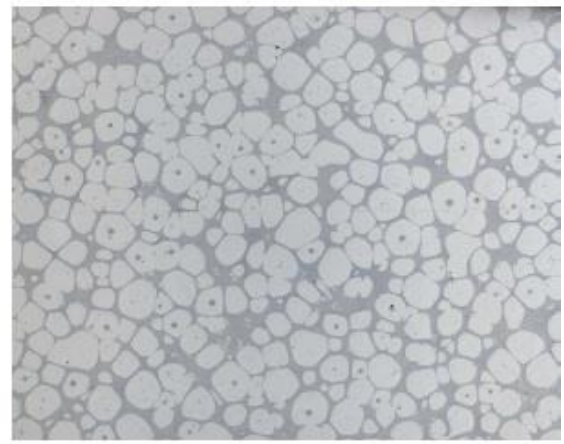

$590{ }^{\circ} \mathrm{C}$

Figure 58 - Al4018 filament micrographs after 15 minutes isothermal holding

Aside from the diameter and form factor the fraction solid was calculated by the analysis software and determined as averaging between $69.6 \%$ and $64.5 \%$ (see Figure 60). This is much higher than expected through the lever rule, where it should be around $50 \%$.

As a last experiment it was reviewed how the filament microstructure will evolve within the first $5 \mathrm{~min}$ of isothermal holding after heating to a now constant temperature of $585^{\circ} \mathrm{C}$ as that was the previously expected temperature for $f_{s}=0.5$.

The results show (see Figure 61) that the microstructure evolution only starts at 5 min where before no change is visible compared to the as-is filament. Even at isothermal holding of 5 min globular grains could not be seen as such the filament would not be in a state for semi-solid processing where that would need globular grains. 


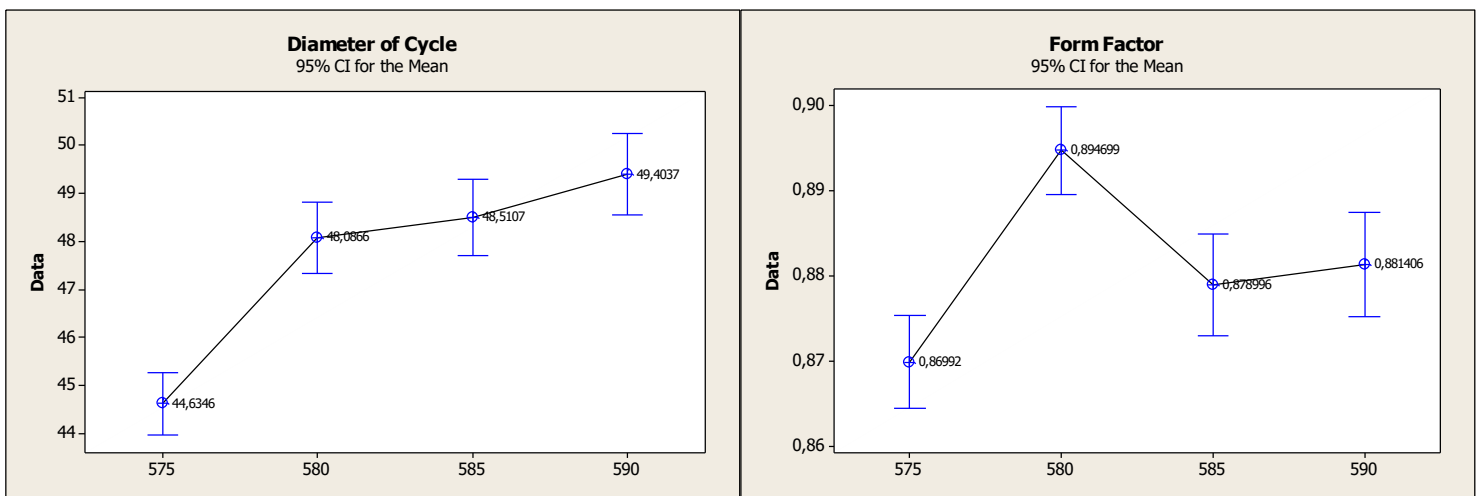

Figure 59 - Graph of average grain diameter and form factor vs. temperature

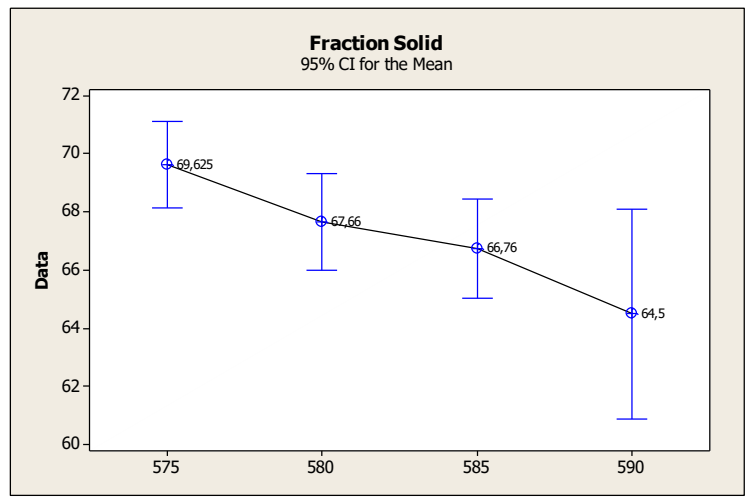

Figure 60 - Fraction solid vs. temperature

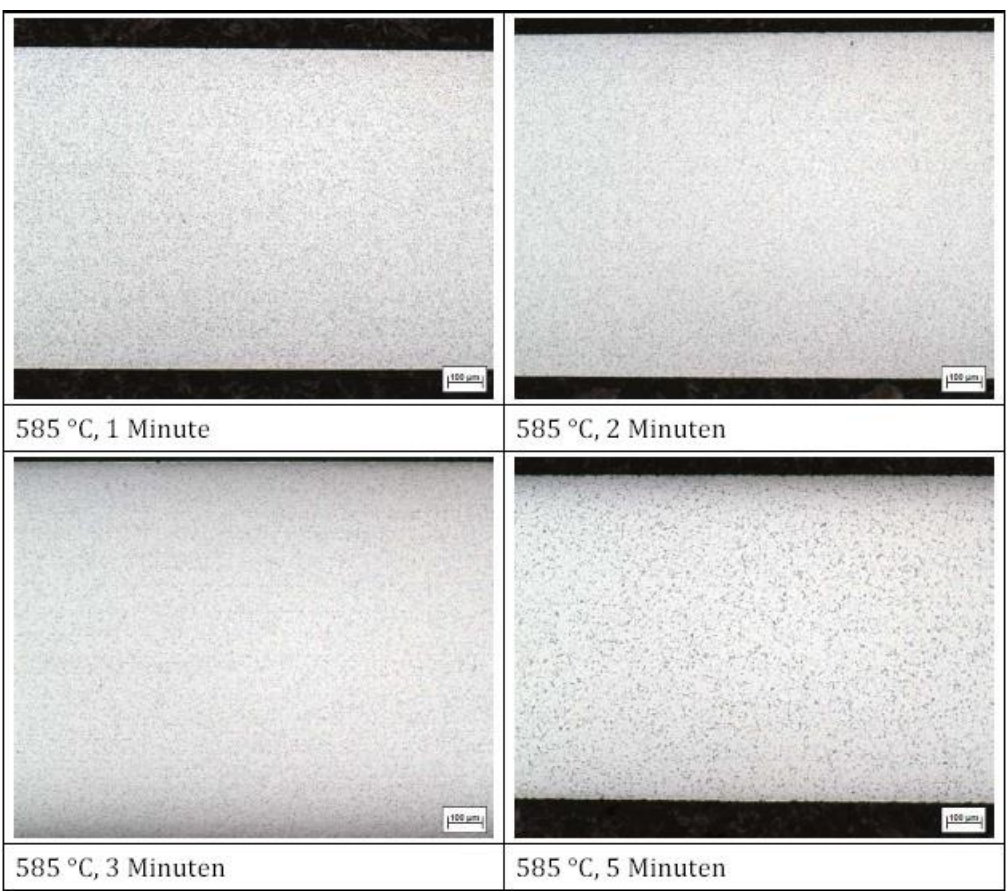

Figure 61 - Microstructure evolution with the first $5 \mathrm{~min}$ of isothermal holding 
Because of the lower fraction solid results as well as the surprisingly late globular microstructure evolution the filament was analyzed for its components via Mass spectrometry (see Figure 62).

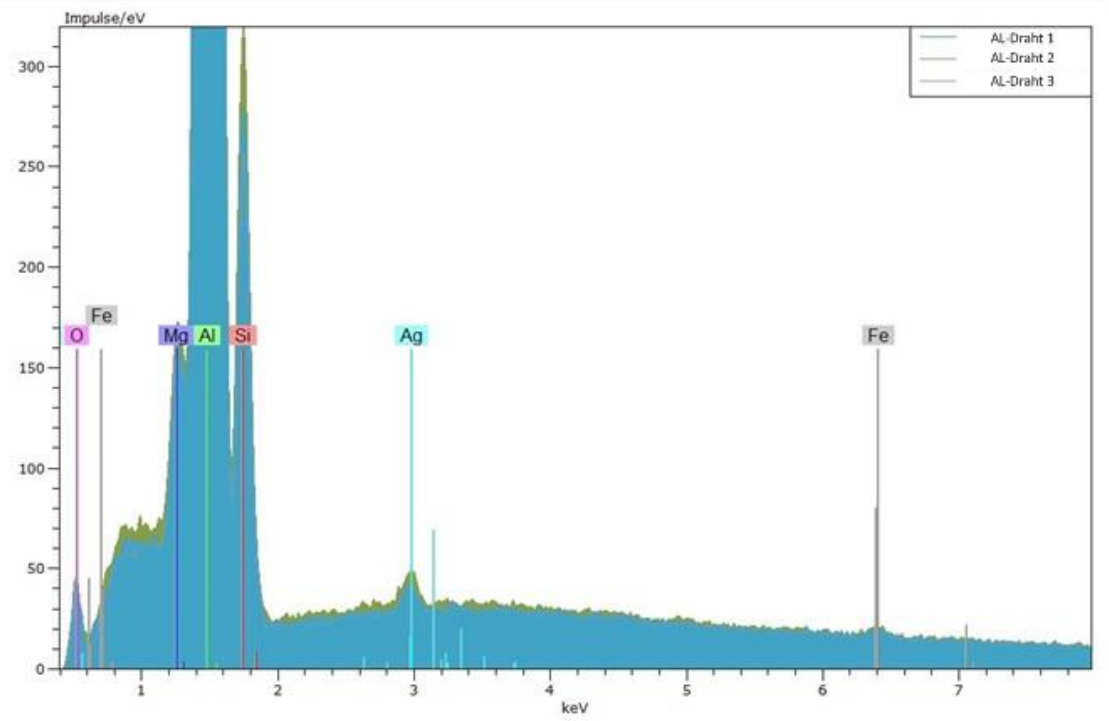

Figure 62 - Mass spectrometry of the Elisental filament

Those results show clearly that the Al4018 standard was not met as the silicon percentage was an average of $4.29 \%$ too low, copper is missing in the alloy, and silver is with almost $0.5 \%$, which is $10 \mathrm{x}$ higher than the allowed additional impurities.

Table 9 - Mass Spectrometry results

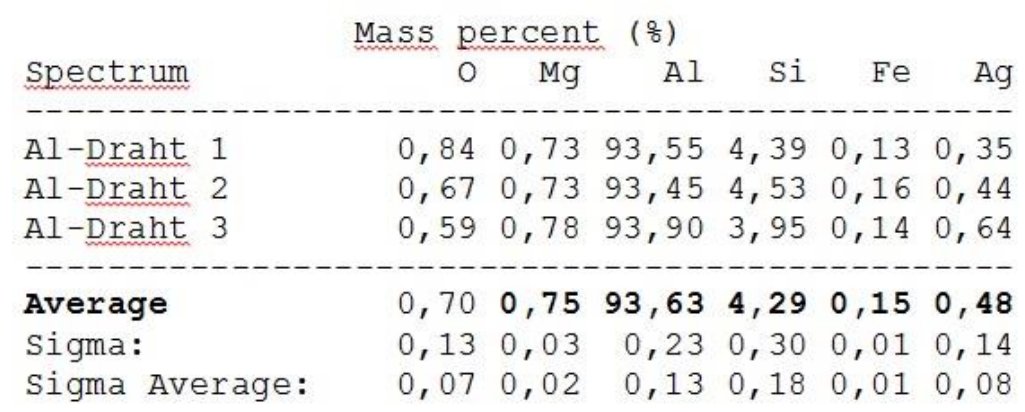

When estimating the filament alloy found in the Elisental filament with a simple Thermo-Calc ${ }^{\circledR}$ calculation for AISi4.29, matching solid fraction rates can be calculated as shown in Figure 63. This would result in a liquidus temperature of $635^{\circ} \mathrm{C}$ and a temperature for the $50 \%$ solid fraction of about $610^{\circ} \mathrm{C}$. 


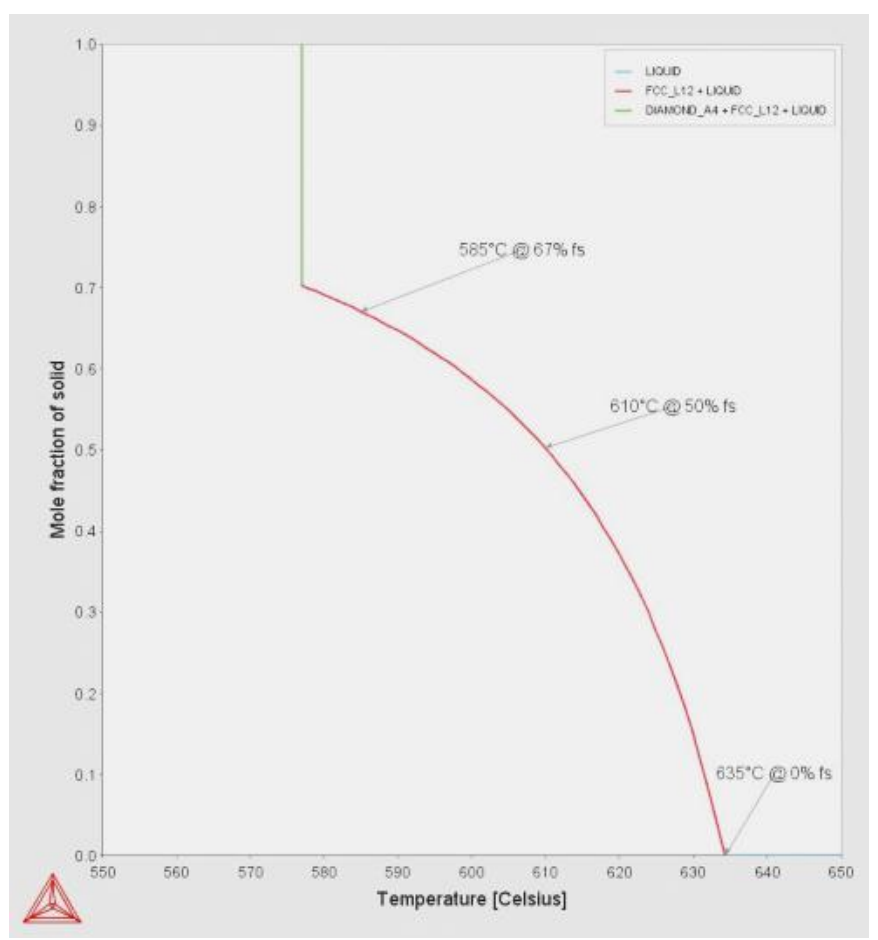

Figure 63 - Estimation of the Elisental filament using Thermo-Calc with Al-Si4.29

The above shows that the processing parameter for the Elisental filament needs to be adapted if used for the semi-solid extrusion. As major elements are missing and the effects of the silver impurity is unknown, this initial experiment did not provide much knowledge or experience related to whether an A356 aluminum alloy can be used without major verification. In other words, the microstructure and thixotropic attributes at about $50 \%$ fraction solid still need to be studied. It has to be evaluated whether production of a real A356 aluminum alloy filament would be more efficient.

\subsection{Metal MEAM test apparatus components and design}

For the execution of extrusion experiments and later 3D object modeling the following components need to be engineered and later integrated:

- a heating channel with either restive or induction heating

- an aluminum filament transport mechanism

- a 3D gantry system

- an oxygen free build chamber

- potentially a full or selective heating device for deposited layer 
For the experiments a nozzle with a high temperature resistive heating channel has been designed by Hotset ${ }^{\circledR}$ Heizpatronen und Zubehör $\mathrm{GmbH}$ (see Figure 64 and Figure 65) using a hotspring ${ }^{\circledR}$ coil heater with $300 \mathrm{~W}$. The company has experience with tin and zinc-based pressure die casting which requires about $100^{\circ} \mathrm{C}$ less processing temperatures than the expected aluminum alloy processing. The nozzles are connected to a control unit with 2 temperature control circuits (see Figure 66).

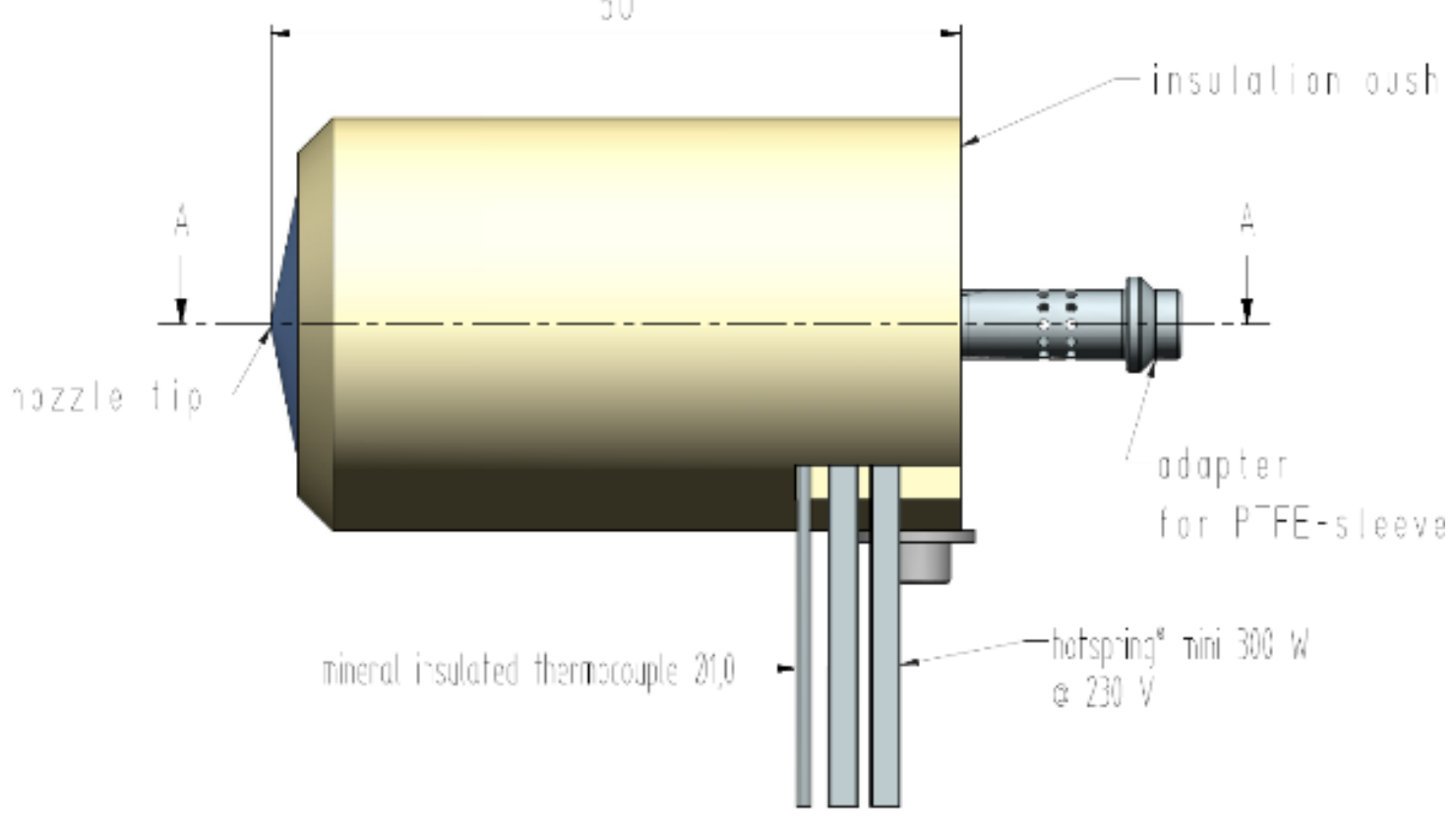

Figure 64 - Hotset $\AA_{\circledast}$ heating nozzle design 


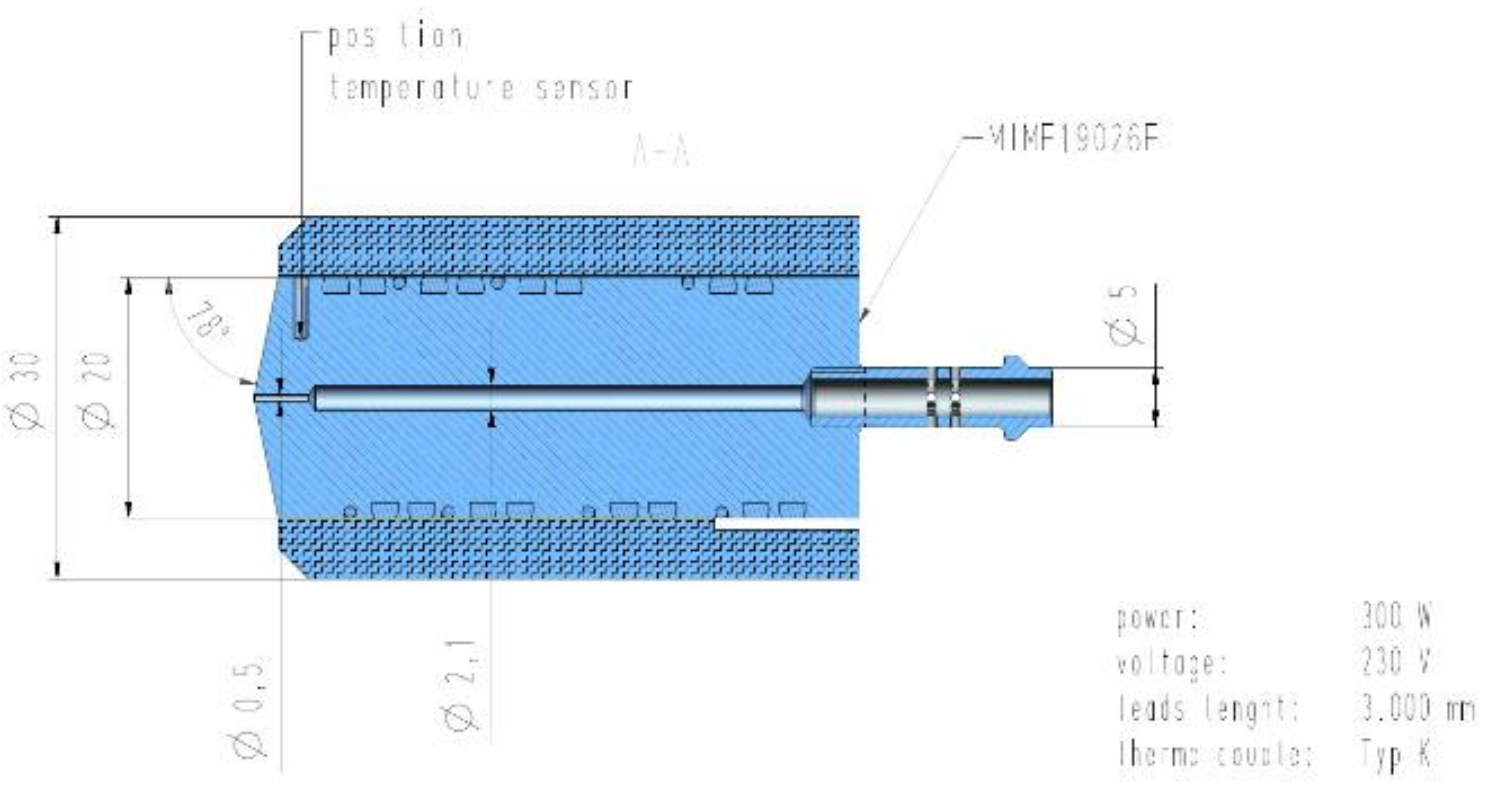

Figure 65 - Hotset ${ }^{\circledR}$ Heating nozzle - sectional view

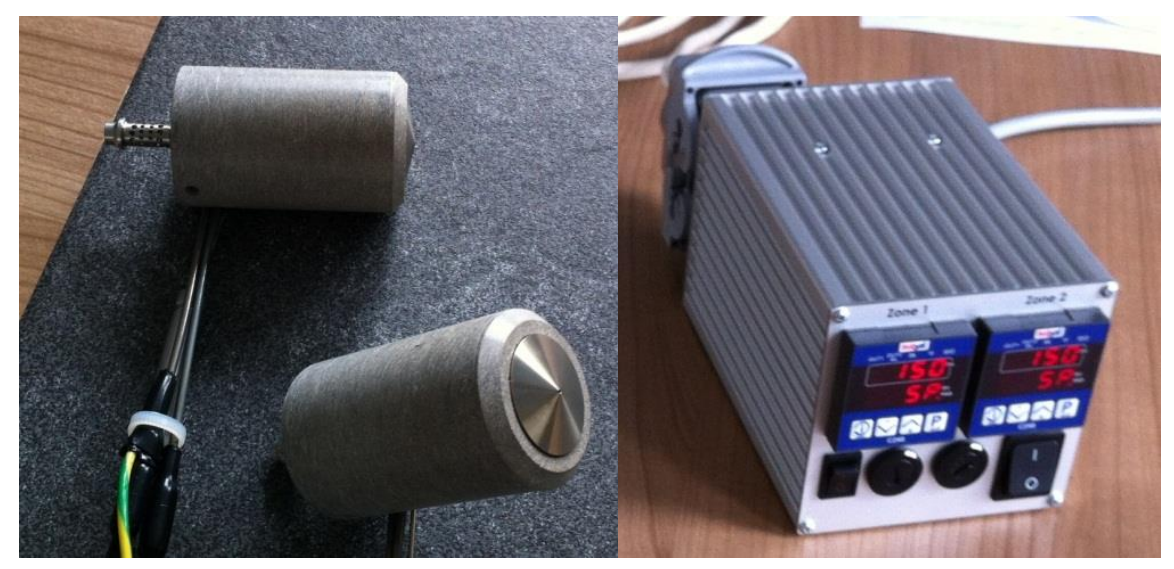

Figure 66 - Resistive heating channel and control unit

It is planned to use the heating channel for extrusion but possibly also for the pretreatment of the filament to allow for microstructure evolution. Later on, one nozzle was improved by adding a replaceable nozzle tip.

For the filament transport a feeding device (see Figure 67) that allows internal slip and external process control has been acquired. The unit is used for filament feeding in robotic soldering installations. 


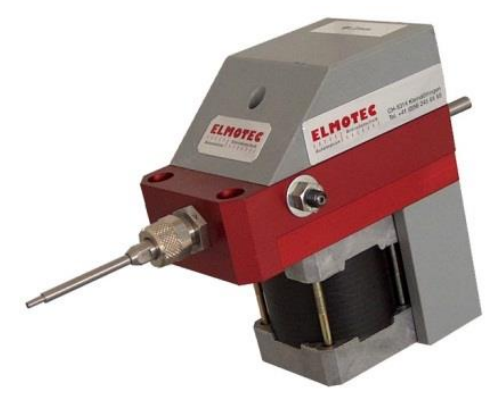

Figure 67 - Wire feed and control unit [118]

A potential 3-dimensional motion system is being realized with an Isel modular CNC base system (see Figure 68). The system is controlled through G-code software, break out board and step motor drivers. For the completion of the first 3D deposition system the wire feed components need to be integrated with the GCode software while the temperature control unit can be separately controlled manually. The 3D motion system has been tested as a rep-strap 22 [119] type 3D printer using $A B S$ and PLA.

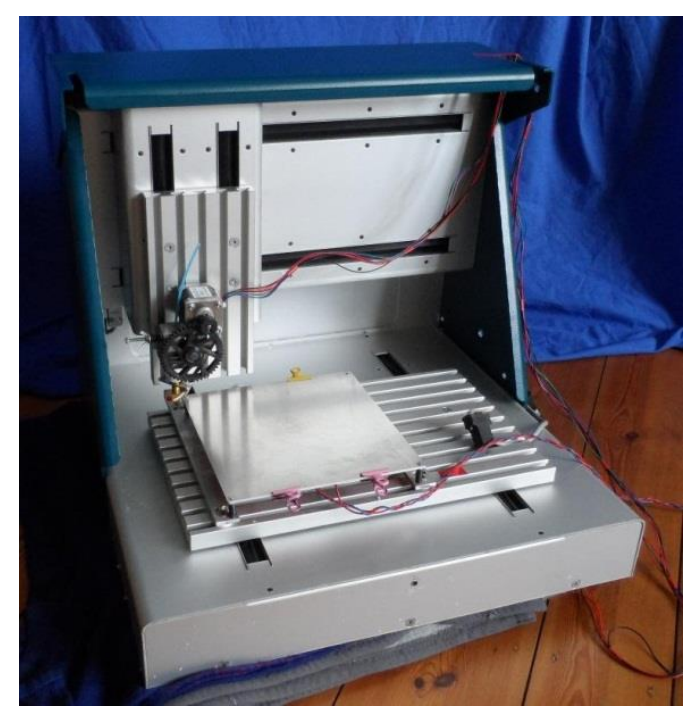

Figure 68 - Isel Modular 3-D motion system

The Isel-Modular will be housed in a closed compartment that can be vented with inert gas e.g. argon. The compartment will be used also for initial extrusion proof of concept without the 3D motion system.

${ }^{22}$ Rep-strap is the term used by the community for re-use of other machines as a rep-rap type 3D printer 
A chamber or selective heating system need to be developed after initial extrusion of several layers and a first bonding review. With the current equipment chosen it is not likely to use a building chamber heating as the components have only a specified working temperature range below $100^{\circ} \mathrm{C}$.

To allow initial heating and extrusion experiments a nozzle mount has been created. It had to be stripped to a relatively thin but stable mount that allows both the nozzle and the nozzle insulation to be held. A pure nozzle mount results in a large temperature loss

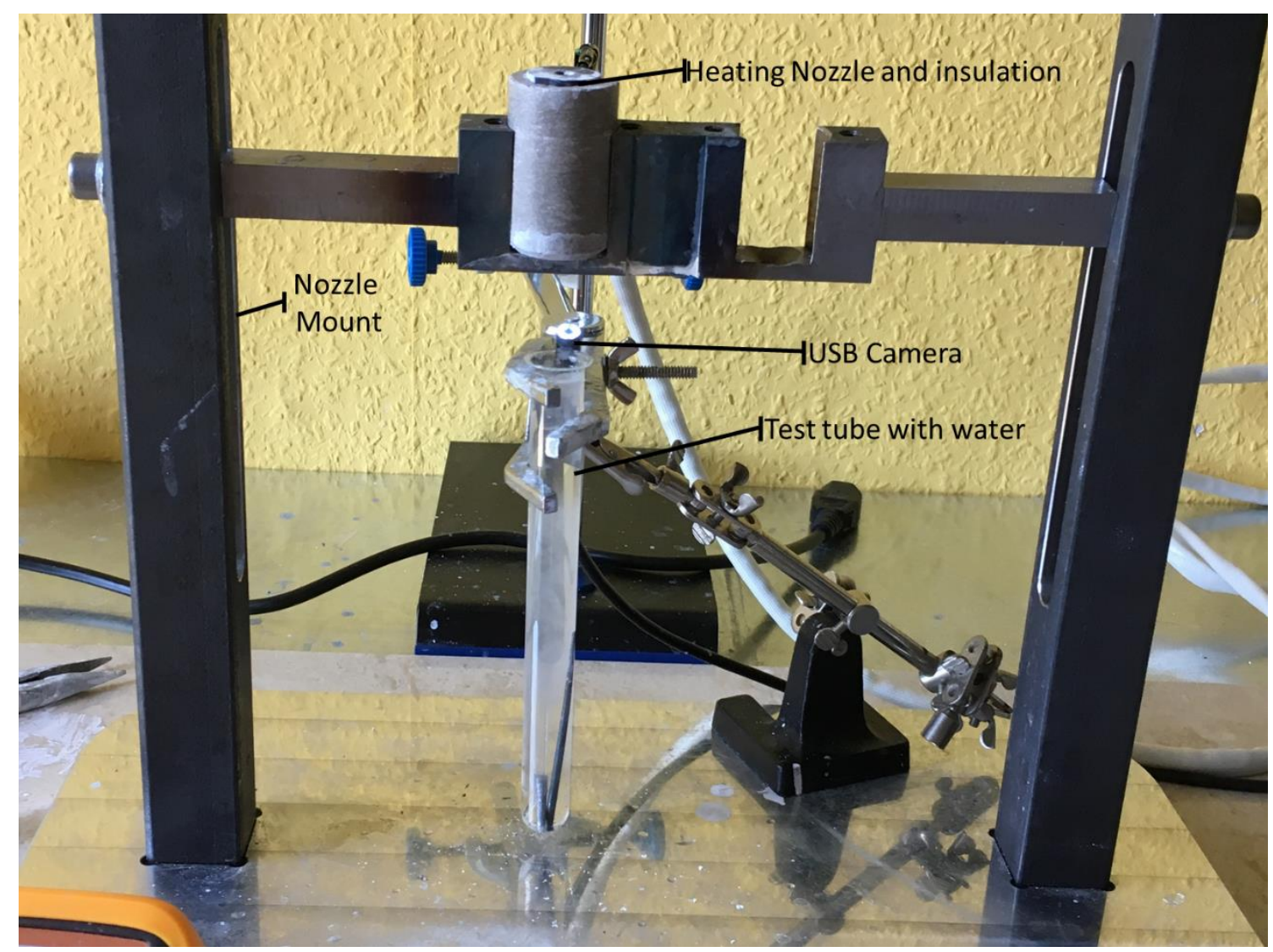

Figure 69 - Filament heating and extrusion test setup

\subsection{Material choice and filament production}

Due to the silver impurity and the missing silicon fraction (see 3.1) it has been decided to use a proper A356 material and one more reference material. In discussion with Salzburger Aluminium AG (Lend/Austria) material has been identified as A356 (THIXALLOY® - 630) and THIXALLOY® - 540 as reference 
material. Both materials are provided in thixo-casting ready state. The company prepares the material through patented extrusion and magnetic stirring process which allows thixotropic processing after a defined inductive heating. Table 10 and Table 11 show the respective composition of the alloy.

Table 10 - A356 (THIXALLOY 630) / AISi7MG0.5 composition (exclusive Al)

\begin{tabular}{|c|c|c|c|c|c|}
\hline $\mathrm{Si}$ & $\mathrm{Fe}(\max )$ & $\mathrm{Cu}(\max )$ & $\mathrm{Mn}(\max )$ & $\mathrm{Mg}$ & $\mathrm{Ti}$ \\
\hline $\mathbf{6 . 5 - 7 . 5}$ & 0.15 & $\max .0 .05$ & $\max .0 .10$ & $\mathbf{0 . 2 5 - \mathbf { 0 . 4 0 }}$ & $0.01-0.15$ \\
\hline
\end{tabular}

Table 11 - THIXALLOY - 540 / AlMg5Si2 composition (exclusive Al)

\begin{tabular}{|c|c|c|c|c|c|c|}
\hline $\mathbf{S i}$ & Fe (max) & $\mathrm{Cu}(\max )$ & $\mathrm{Mn}$ & $\mathrm{Mg}$ & $\mathrm{Zn}$ (max) & $\mathrm{Ti}$ \\
\hline $\mathbf{1 . 8 - 2 . 6}$ & 0.20 & 0.02 & $\mathbf{0 . 2 5}$ & $\mathbf{5 . 0 - 6 . 0}$ & 0.07 & $0.01-0.05$ \\
\hline
\end{tabular}

The raw material is delivered in form of $76 \mathrm{~mm}$ and $88 \mathrm{~mm}$ diameter rods each $6 \mathrm{~m}$ long (see Figure 70a). As a preparation of the filament extrusion the rod material has been trimmed down to bolts of $28 \mathrm{~mm} \times 100 \mathrm{~mm}$ size (see Figure $70 \mathrm{~b}$ ).

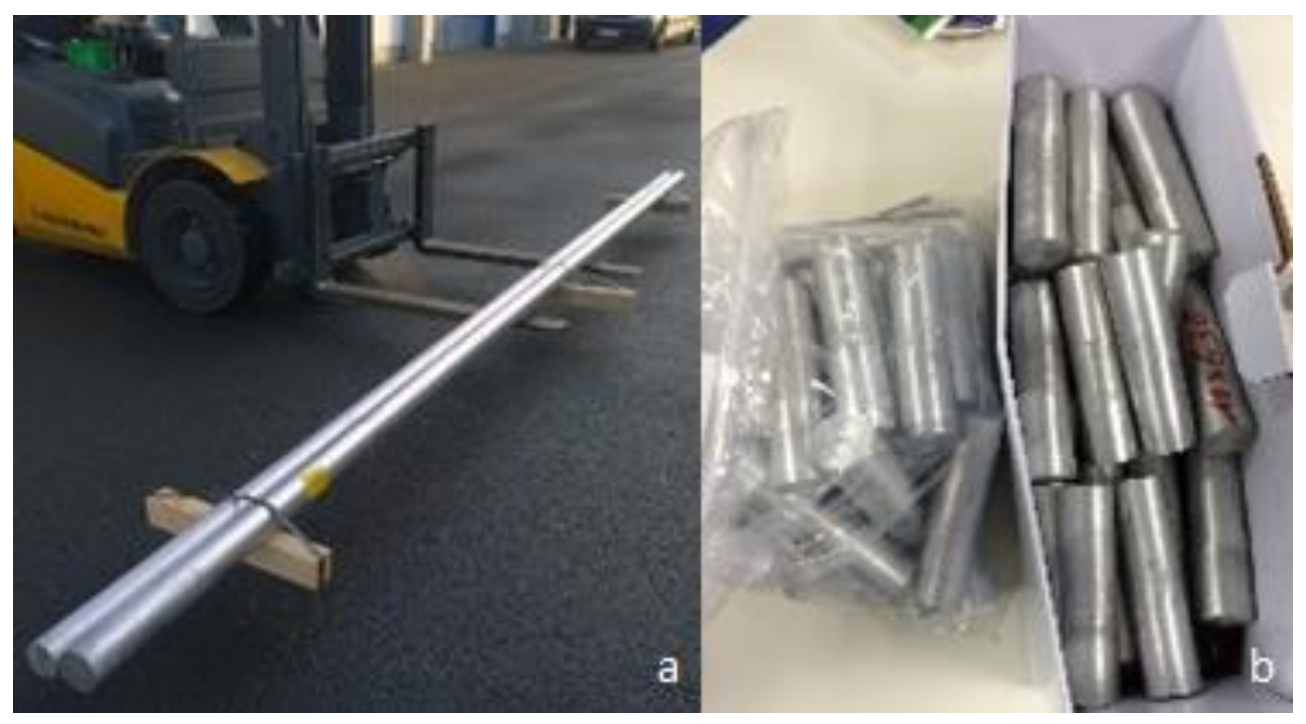

Figure 70 - Raw material (a - rod, b - reduced bolt)

Filament has been produced with a 0.5MN Extrusion press (see Figure 71 a) with the parameter as shown in Figure $71 \mathrm{~b}$ and Table 12. A problem identified during the filament production was the adhesive tendency of the A356 alloy. This resulted 
in clogging and cleaning several times. A small sample of THIXOALLOY - 540 has been produced as well using similar processing parameters. The adhesive tendency was not as strong as with A356.

Table 12 - Filament extrusion process parameter

\begin{tabular}{|c|c|c|c|c|c|c|c|c|c|}
\hline \multirow[t]{2}{*}{ Material } & \multicolumn{2}{|c|}{ Bolt } & \multirow{2}{*}{$\begin{array}{l}\text { Temp } \\
\text { T_B } \\
{ }^{\circ} \mathbf{C}\end{array}$} & \multicolumn{2}{|c|}{$\begin{array}{c}\text { Press } \\
\text { Cylinder }\end{array}$} & \multicolumn{2}{|c|}{ Die } & \multirow{2}{*}{$\begin{array}{c}\text { Ratio } \\
\text { [-] }\end{array}$} & \multirow{2}{*}{$\begin{array}{c}\text { Ram } \\
\text { speed } \\
\text { V } \\
\mathrm{mm} / \mathrm{s}\end{array}$} \\
\hline & $\begin{array}{l}\text { D_B } \\
\mathrm{mm}\end{array}$ & $\begin{array}{l}\mathrm{L} \text { in } \\
\mathrm{mm}\end{array}$ & & $\begin{array}{c}\text { D_C } \\
\mathrm{mm}\end{array}$ & $\begin{array}{c}\text { T_C } \\
{ }^{\circ} \mathbf{C}\end{array}$ & $\begin{array}{l}\text { D_D } \\
\mathrm{mm}\end{array}$ & $\begin{array}{c}\text { T_D } \\
{ }^{\circ} \mathbf{C}\end{array}$ & & \\
\hline A356 & 28 & 100 & 420 & 30 & 400 & $2(4 x)$ & 420 & 56 & 3 \\
\hline
\end{tabular}
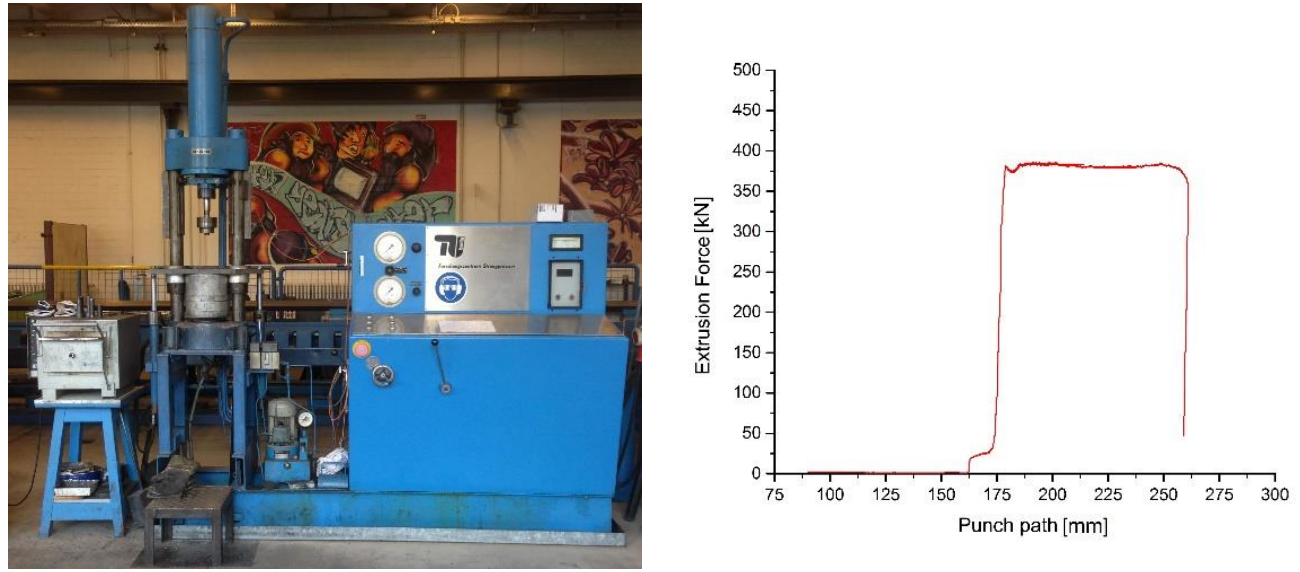

Figure $71-0.5 \mathrm{MN}$ extrusion press and recorded processing parameter

To understand the microstructure before and after a micrograph analysis has been conducted. In general, dendritic microstructure of the raw material are dissolved after extrusion. See Figure 72, Figure 73, Figure 74, Figure 75 for micrograph pictures and Table 13 for analysis results.

Table 13 - Microstructure analysis results of raw and extruded material

\begin{tabular}{|l|lc|ll|}
\hline Material & A356 & THIXALLOY 540 & \\
\hline Raw Rod & $\begin{array}{l}\text { Dendritic structure with } \\
\text { globular grain }\end{array}$ & $\begin{array}{l}\text { Dendritic structure with } \\
\text { globular grain }\end{array}$ & \\
\hline
\end{tabular}




\begin{tabular}{|c|c|c|}
\hline & $\begin{array}{l}\text { - Scattered precipitates up to } \\
2 \mu \mathrm{m} \text { size within dendrite } \\
\text { arms }\end{array}$ & $\begin{array}{l}\text { - Scattered precipitates up to } \\
2 \mu \mathrm{m} \text { size at dendritic border } \\
\text { and between dendrite arms }\end{array}$ \\
\hline Extruded & $\begin{array}{ll}\text { - } & \text { Structure dissolved } \\
\text { - } & \text { Homogenous precipitates }\end{array}$ & $\begin{array}{ll}\text { - } & \text { Structure dissolved } \\
\text { - } & \text { Homogenous precipitates } \\
\text { - } & \text { Individual coarse precipitates }\end{array}$ \\
\hline
\end{tabular}

a)

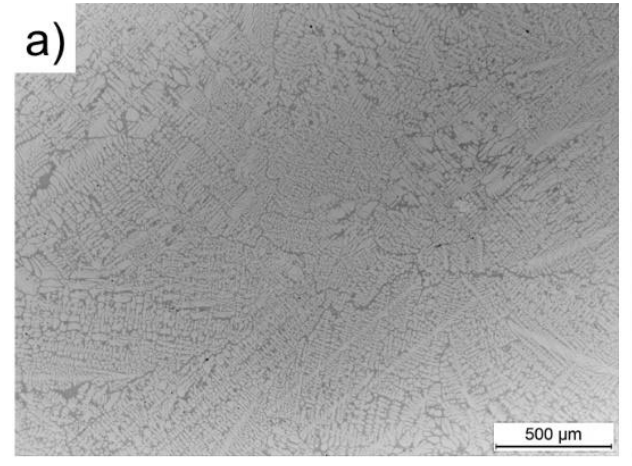

c)

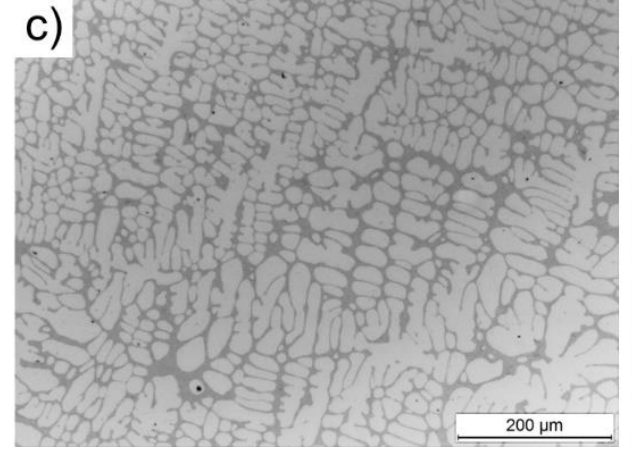

b)

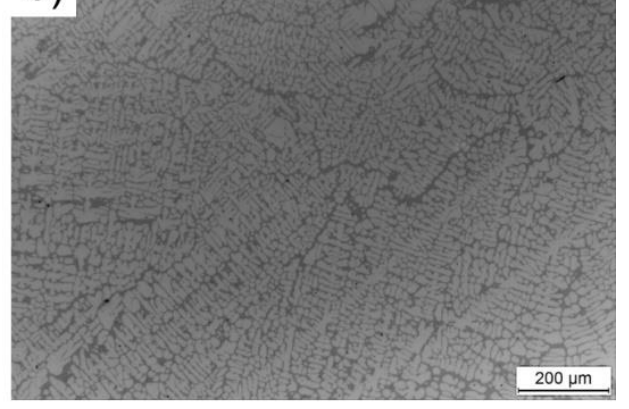

d)

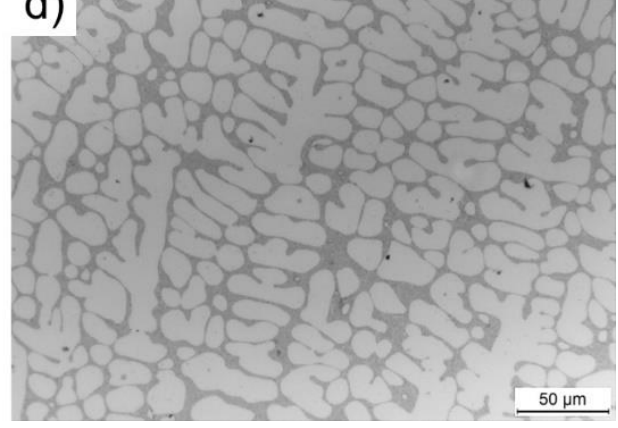

Figure 72 - A356 (THIXALLOY 630) raw rod material a) 25x magnified b) 50x magnified c) 50x magnified d) 200x magnified 

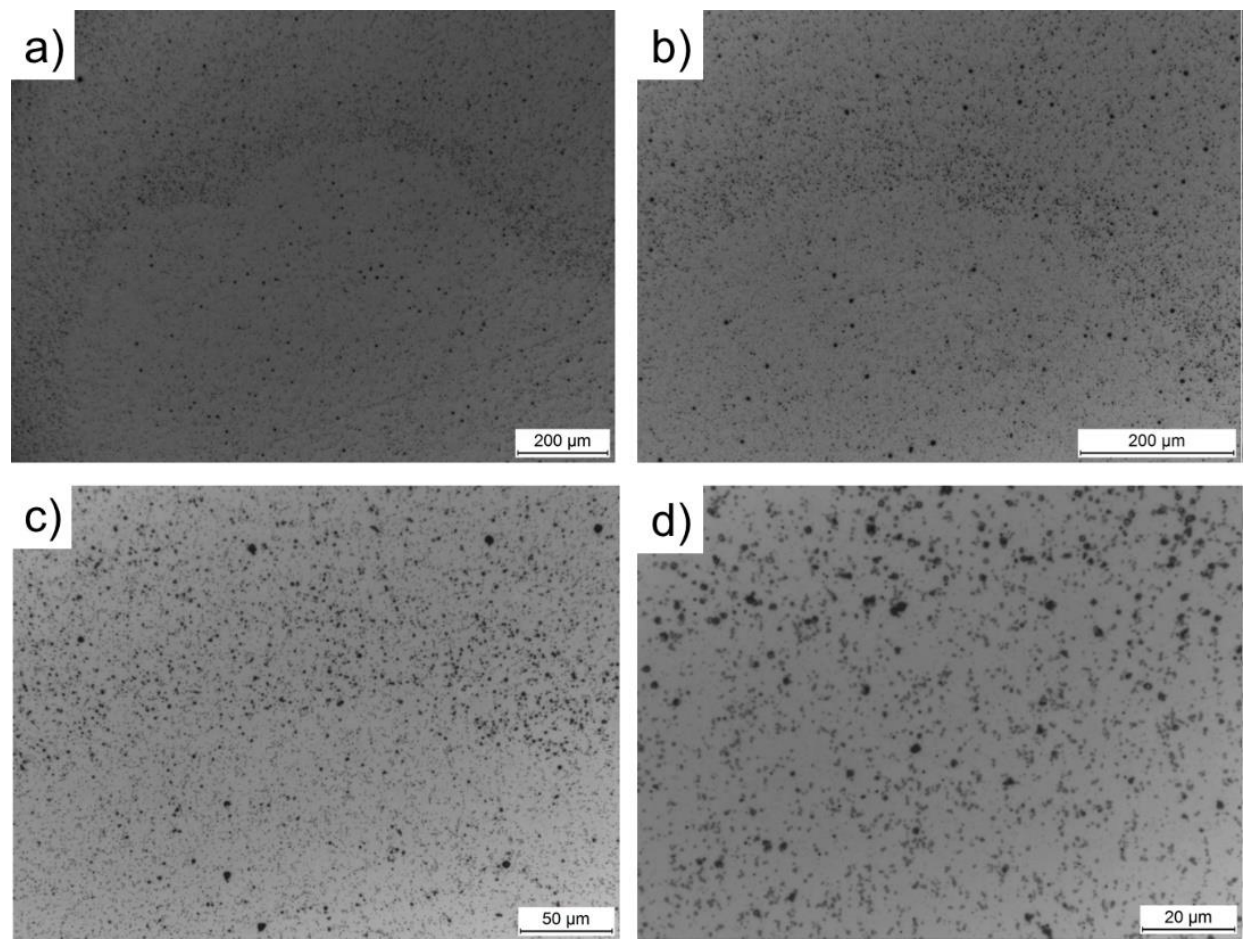

Figure 73 - A356 extruded filament a) 50x magnified b) 100x magnified c) 200x magnified d) 500x magnified
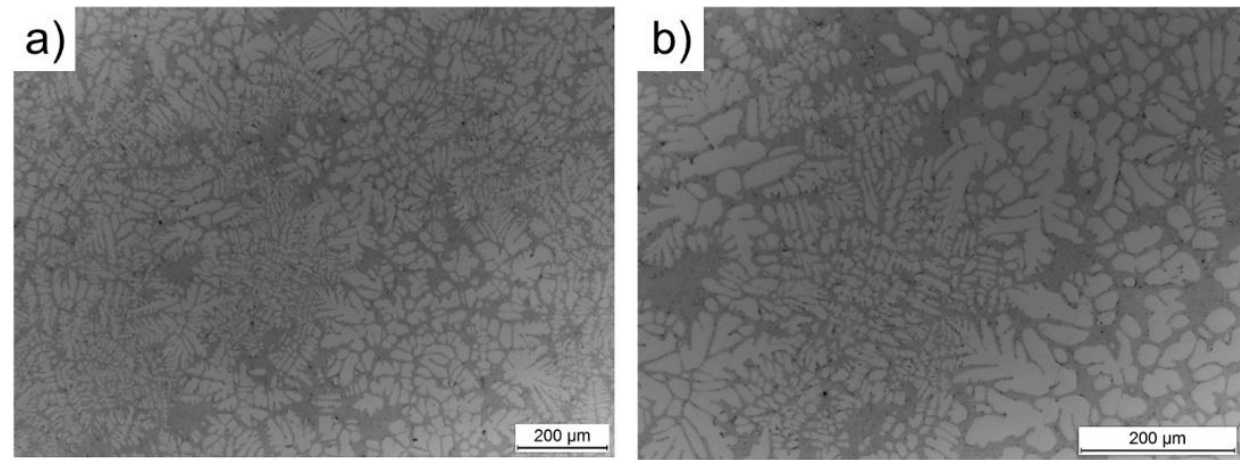

c)
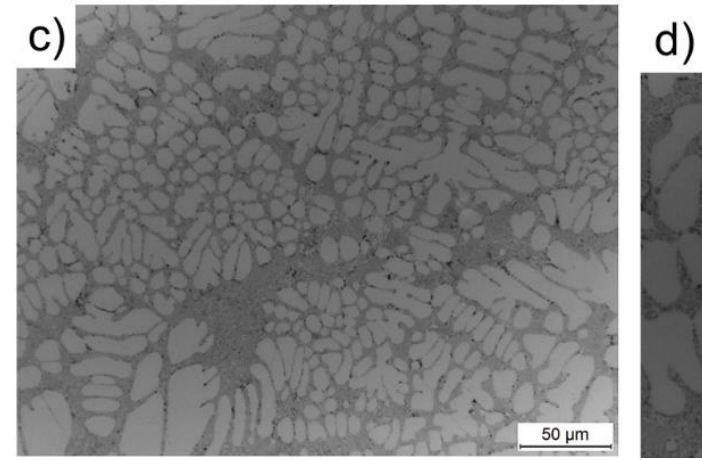

d)

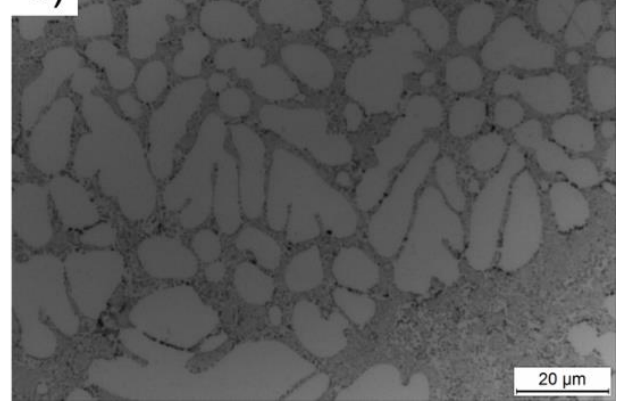

Figure 74 - THIXALLOY 540 raw rod material a) 50x magnified b) 100x magnified c) 200x magnified d) 500x magnified 
a)
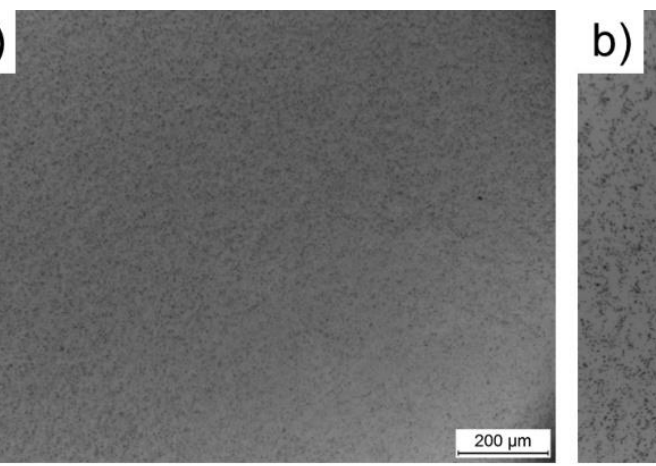

c)

)

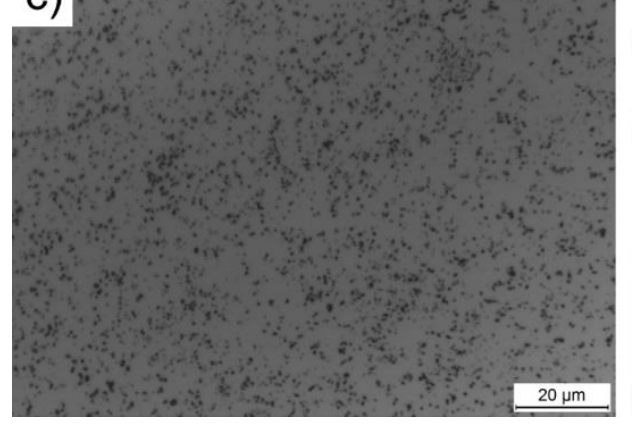

Figure 75 - THIXALLOY 540 extruded filament a) 50x magnified b) 100x magnified c) 200x magnified d) 500x magnified

\subsection{Initial proof of concept testing}

With the availability of new filament material (A356) a proof of concept setting was built using the heating nozzle, the wire feeder and a housing with inert argon gas (see Figure 76).

The apparatus was tested in terms of heating up to $650^{\circ} \mathrm{C}$ to allow liquid extrusion if needed. The wire feeder was tested with the heating channel i.e. removing the nozzle tip from the enhanced nozzle.

Extrusion and heating experiments with A356 and the Al4018 resulting in clogging and adhesion to the nozzle wall. No controlled extrusion was possible. 


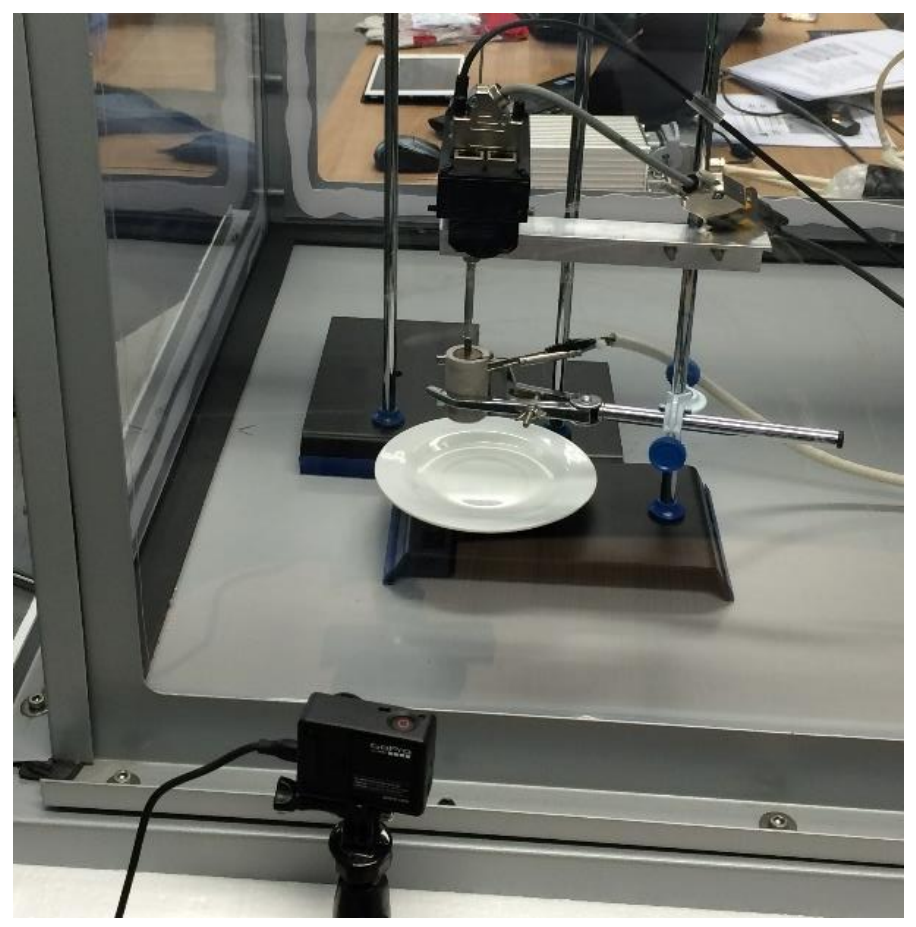

Figure 76 - Proof of concept extrusion setting

This failed proof of concept showed that the material and process parameter need to be closely controlled. As planned prior the following reviews need to be performed:

- Temperature management in the resistive nozzle and resulting liquid fraction of the alloy - Simulation

- Compare temperature management through inductive heating as used in semi-solid casting - Simulation

- Microstructure evolution - Experiments

- Microstructure time dependency though coarsening (aging) - Experiments 


\section{SIMULATION AND EXPERIMENT}

\subsection{Simulations}

\subsubsection{Simulation of heated channel changes}

To understand the heating channel behavior under semi sold processing condition a thermal analysis simulation has been conducted with SolidWorks. Important for the extrusion process and design dimension of the nozzle is how much the dimension of the heating channel changes from ambient $\left(20^{\circ} \mathrm{C}\right)$ to semi-solid temperature $\left(585^{\circ} \mathrm{C}\right)$. This is especially important regarding potential segregation and back-extrusion of the liquid phase. The expansion of the nozzle was observed during commissioning of the Nozzle from Hotset. During delivery testing the insulation cracked at about $350^{\circ} \mathrm{C}$ (see Figure 77). The supplier replaces both insulations and determined the incorrect expansion calculation and therefore required larger inner diameter of the insulation as the cause.

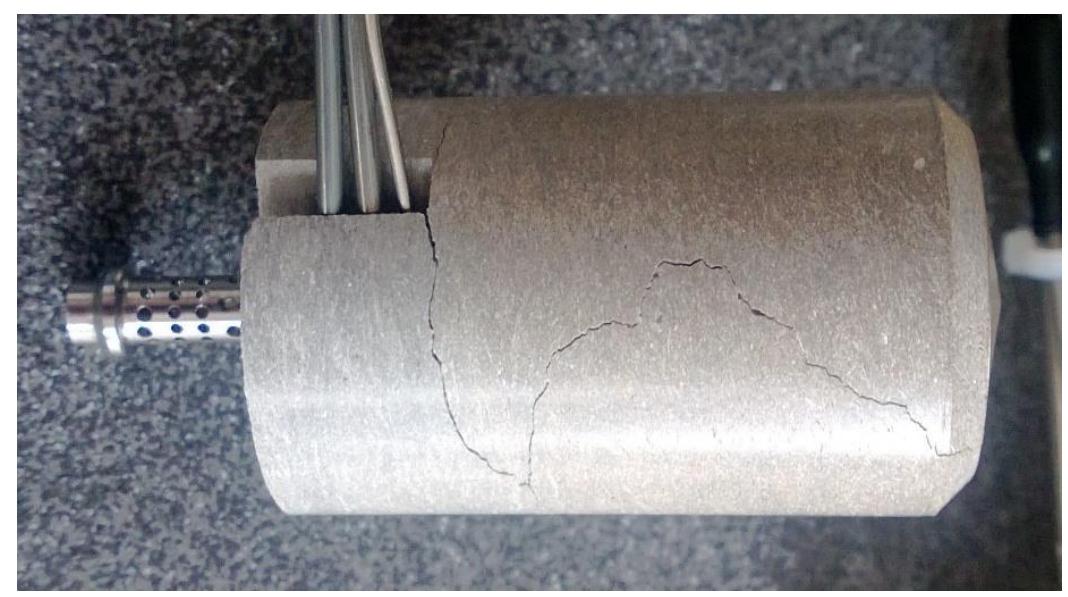

Figure 77 - Cracked nozzle insulation due to expansion at elevated temperature

While the temperature for this simulation is assumed uniform (at $585^{\circ} \mathrm{C}$ ) the displacement vector (URES) shows the highest value at the nozzle top and bottom (see Figure 78c). The dimension of the heating channel changes by about $1 \%$ from designed $2.1 \mathrm{~mm}$ to $2.12 \mathrm{~mm}$. 

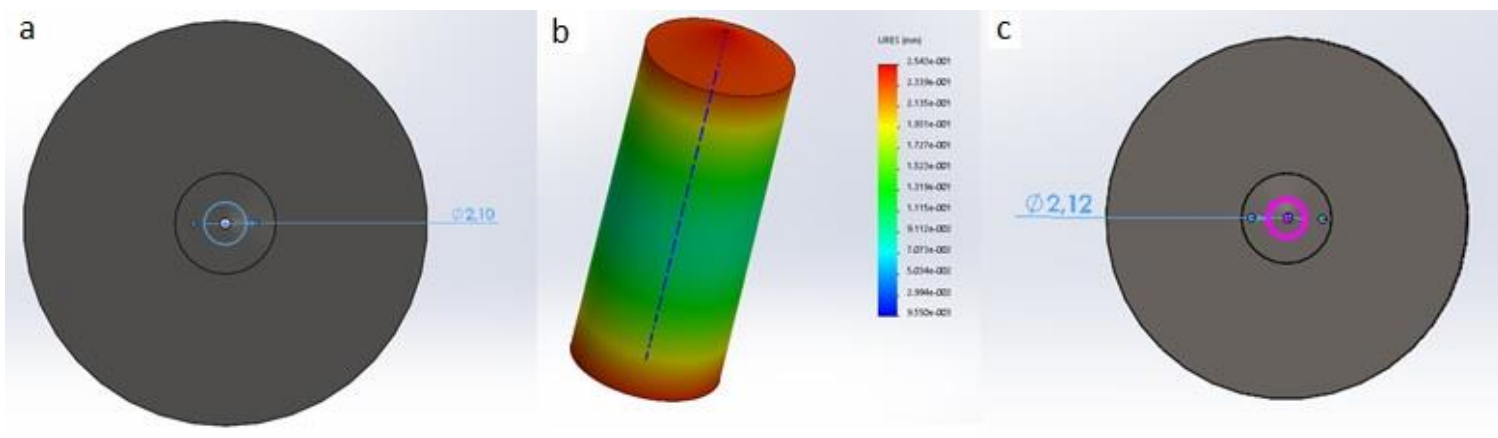

Figure 78 - Heating channel simulation a) nozzle entry dimension at ambient temperature b) displacement vector at $585^{\circ} \mathrm{C}$ c)nozzle entry dimension at $585^{\circ} \mathrm{C}$

\subsubsection{Simulation of resistive heating}

To understand the heating of the filament in a resistive heating channel a simulation has been conducted with Ansys Fluent. As the material liquifies through the heating process it is important to model the filament material correctly in the simulation. For that purpose, linear approximation of calculated values from JMatPro V8 for the fluent model of A356 have been used.

Besides the temperature dependent density (see Figure 79) especially the specific heat required to increase the temperature by $1 \mathrm{~K}$ (see Figure 80 ) as well as the thermal conductivity (see Figure 81) were reviewed and piecewise linear approximations are defined. For completeness of the material model for Fluent the approximated total viscosity (see Figure 82 ) has been included as well. Note that this viscosity will change with the dendritic or globular microstructure (see section 2.1.5 and Figure 9). As the heating simulation does not include an actual nozzle but just a heating channel this can be neglected. The material model is completed by the solidus temperature $\left(555^{\circ} \mathrm{C} / 828.15 \mathrm{~K}\right)$, the liquidus temperature $\left(615^{\circ} \mathrm{C} / 888.15 \mathrm{~K}\right)$ and the pure solvent melting heat or heat of fusion $(389000 \mathrm{~J} / \mathrm{Kg})$. This more accurate material model was used throughout the simulations. 


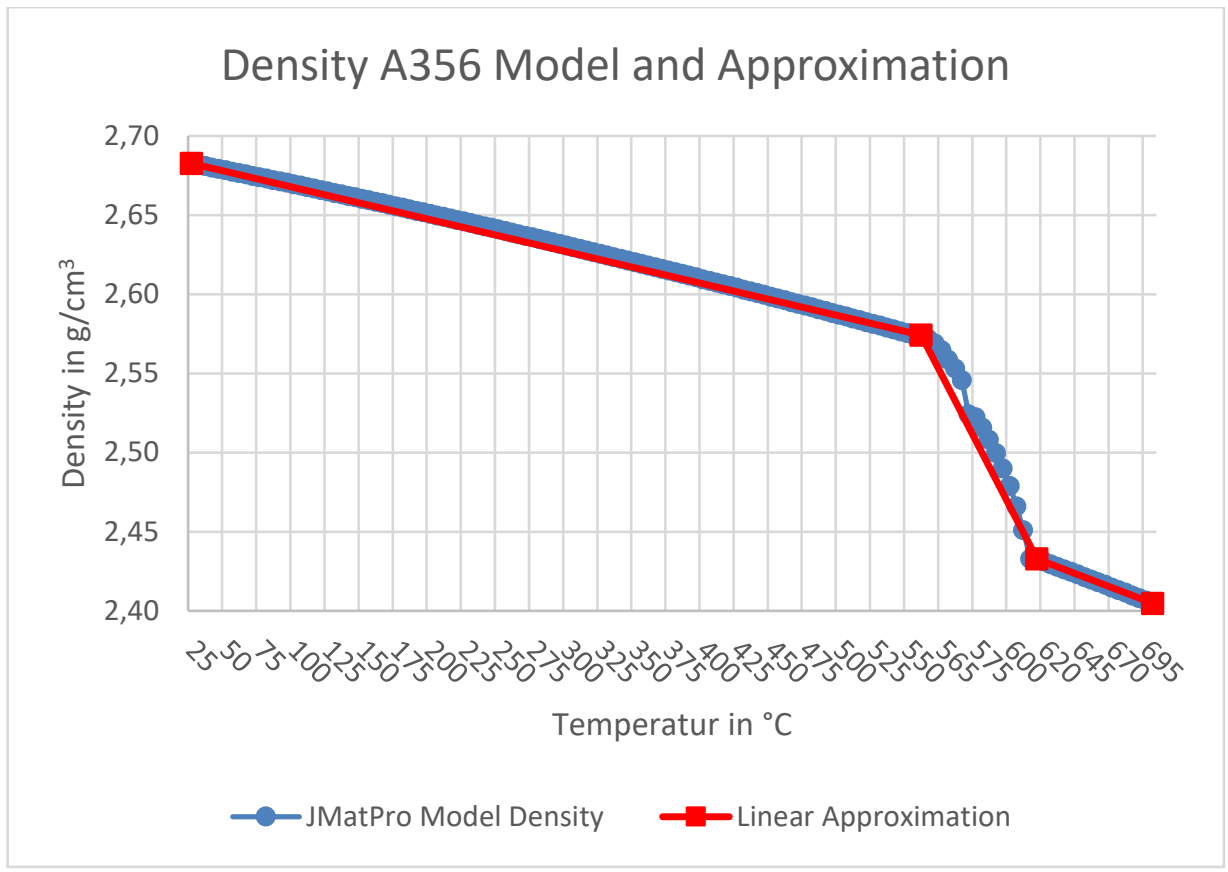

Figure 79 - A356 Density / Temperature model and approximation

Specific Heat A356 Model and Approximation

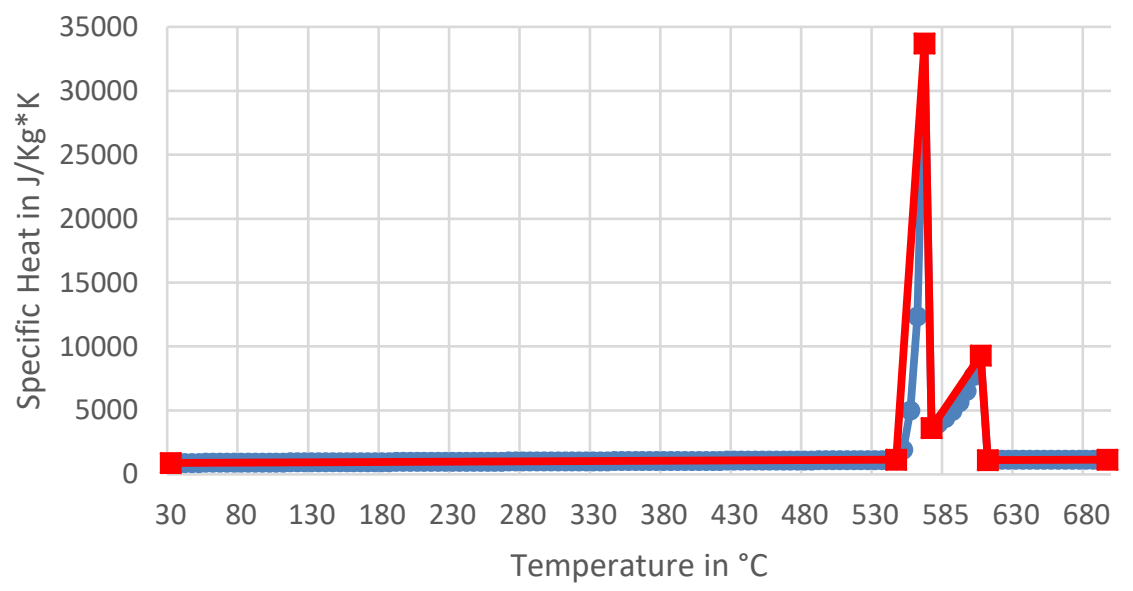

-JMatPro Model Specific Heat $\quad$ Linear Approximation

Figure 80 - A356 Specific Heat / Temperature model and approximation 


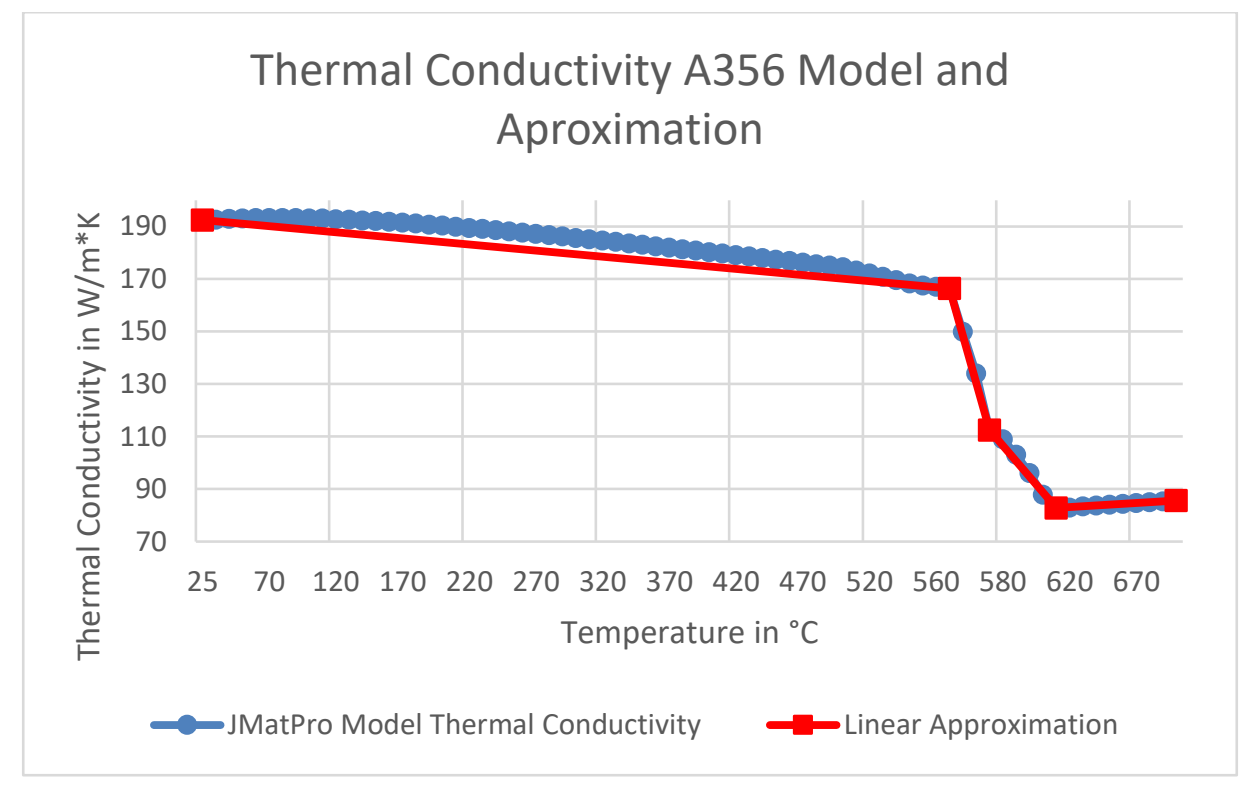

Figure 81 - A356 Thermal Conductivity / Temperature model and approximation

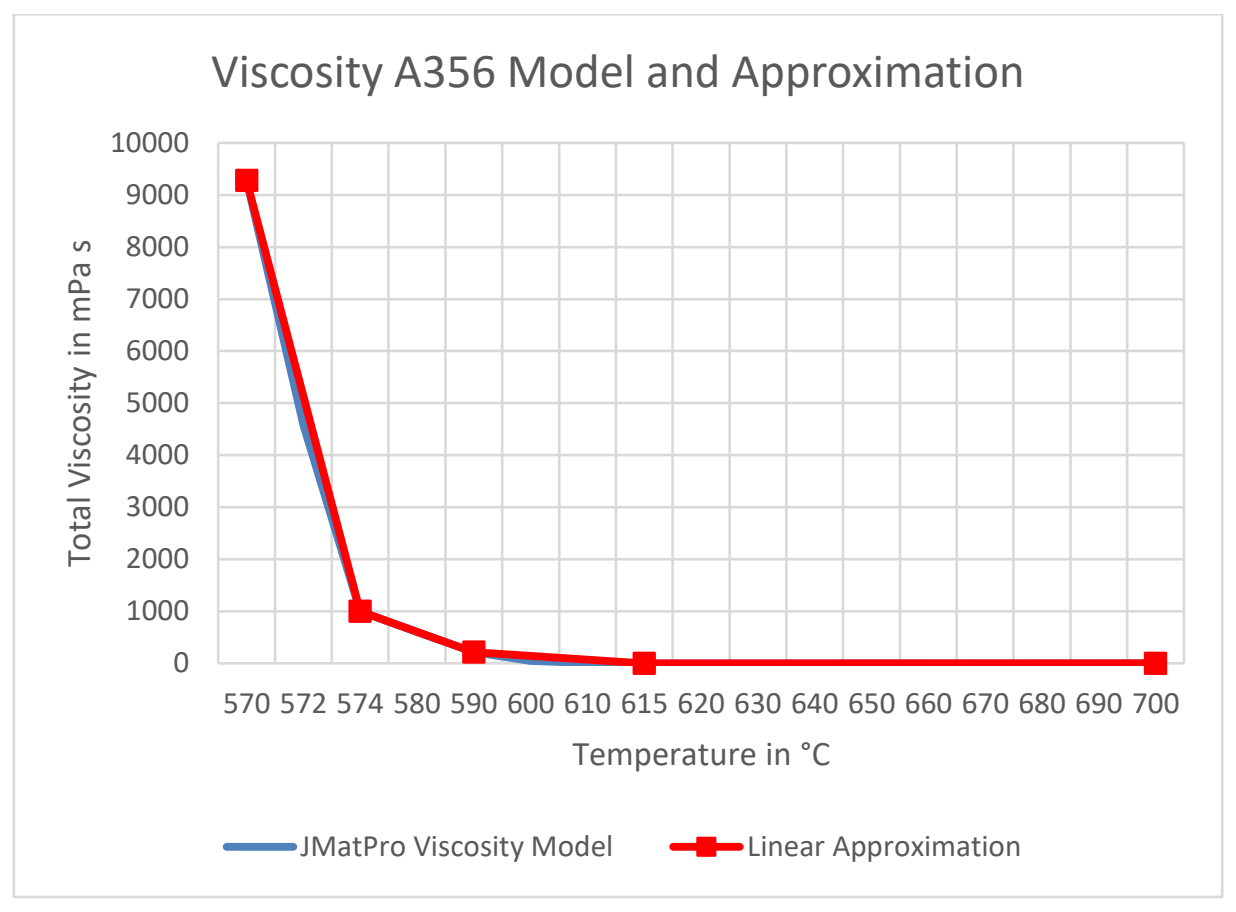

Figure 82 - A356 Viscosity at liquid temperatures model and approximation

\subsubsection{Ideal Nozzle}

The initial resistive heating simulation was performed using an ideal uniform temperature distribution in the nozzle wall heated by the resistive coil heaters at 
the outside wall of the nozzle (see Figure 83 for actual nozzle and Figure 64/Figure 65 for design).

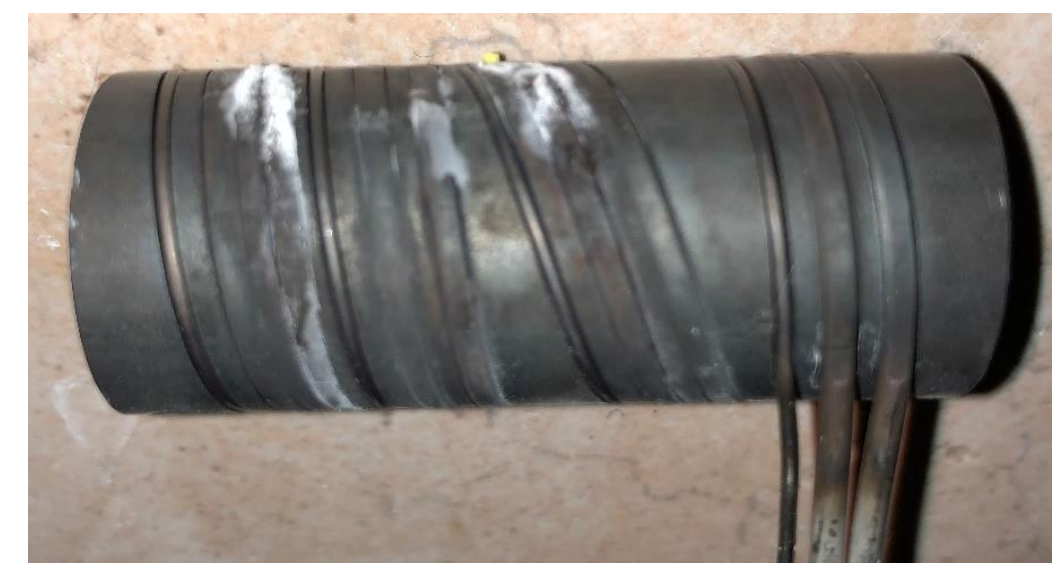

Figure 83 - Nozzle inside with resistive coil heaters and thermocouple connection

With the material settings for $\mathrm{A} 356$ and the ideal nozzle approach (uniform $585^{\circ} \mathrm{C}$ for $\left.50 \% \mathrm{FS} / \mathrm{FL}_{\mathrm{L}}\right)$ several inlet filament velocities have been simulated $(0.1 \mathrm{~mm} / \mathrm{s}$, $1 \mathrm{~mm} / \mathrm{s}, 2 \mathrm{~mm} / \mathrm{s}$ ). With $2 \mathrm{~mm} / \mathrm{s}$ the simulation showed the maximum velocity still achieving the $585^{\circ} \mathrm{C} / 50 \%$ fraction solid/liquid (see Figure 85 ).

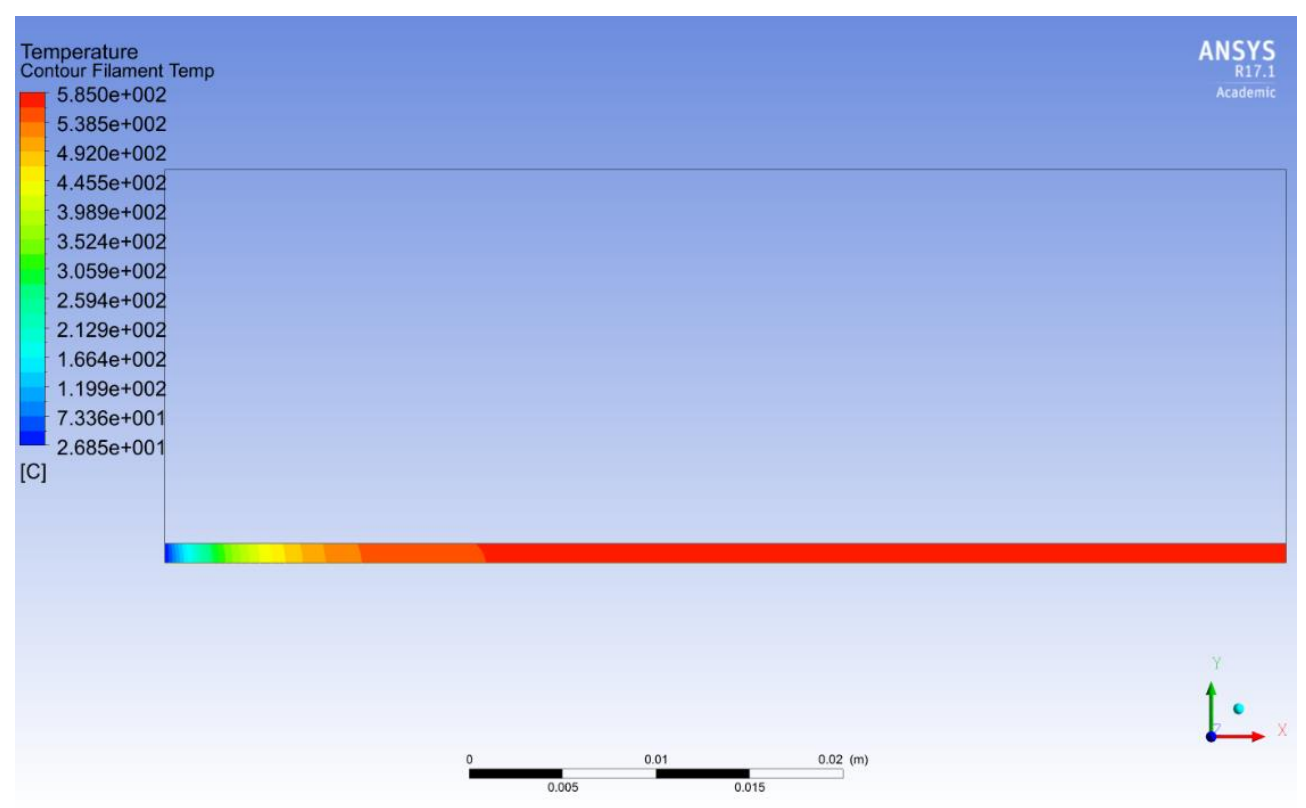

Figure 84 - Temperature contour for ideal/uniform nozzle temperature at $585^{\circ} \mathrm{C}, 2 \mathrm{~mm} / \mathrm{s}$ 

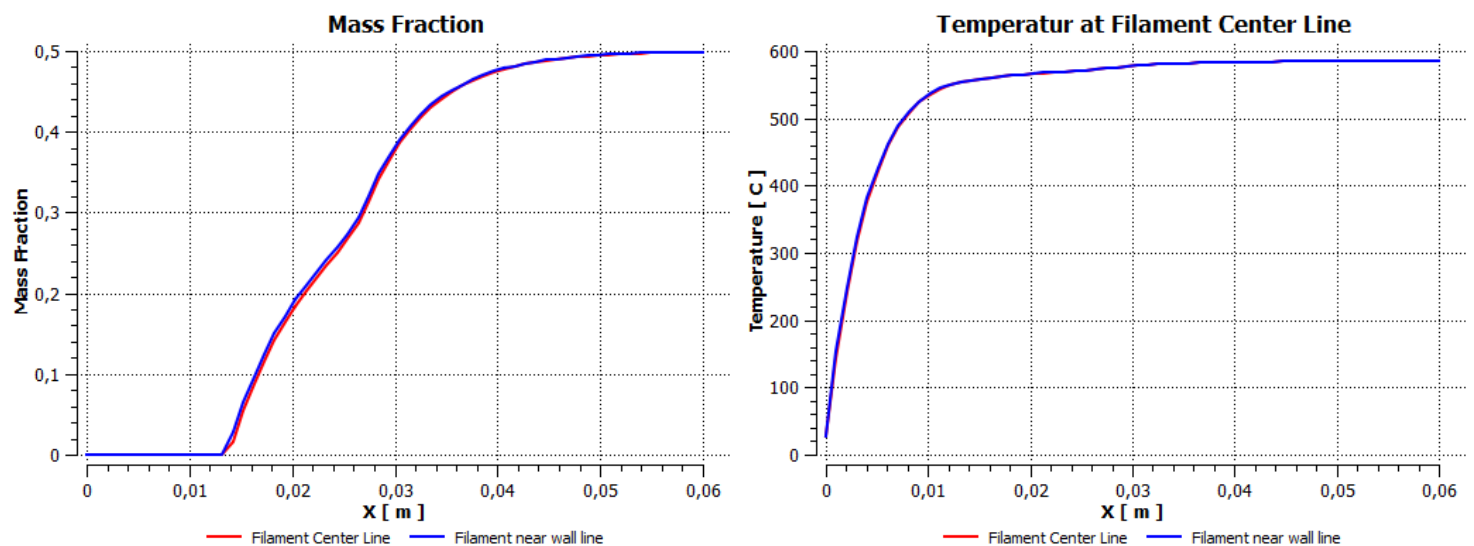

Figure 85 - A356 Simulation results for ideal/uniform nozzle temperature at $585^{\circ} \mathrm{C}, 2 \mathrm{~mm} / \mathrm{s}$ Mass fraction liquid and temperature

As shown in Figure 83 the resistive heating coils are not uniformly distributed throughout the nozzle wall. As such a non-uniform temperature distribution within the nozzle is expected. The next step in simulating the real nozzle is to identify the temperature distribution.

\subsubsection{Nozzle temperature verification}

To identify the distribution function in the nozzle the temperature has been experimentally recorded. Due to the nozzle temperature fluctuation as well as expected measurement error (measurement depth, temperature, etc.), design of experiment was used (see section 0 ).

After definition of 2 factors ( 2 temperature setting levels, 8 sensor depth levels) and a pre-experiment of 2 samples were conducted resulting in a full factorial design of 80 runs. The results (see Figure 86 and section 0 ) show an inadequacy of a linear model for the temperature distribution (calculated as delta temperature $=$ actual temperature - setting temperature).

For purpose of the simulation the following temperature profile function has been determined for $\mathrm{A} 356$ and $585^{\circ} \mathrm{C}$ setting through a fitted line of the average values determined by the experiment (see Figure 87):

$$
T(x)=702.5+8545 * x-108048 * x^{2}
$$




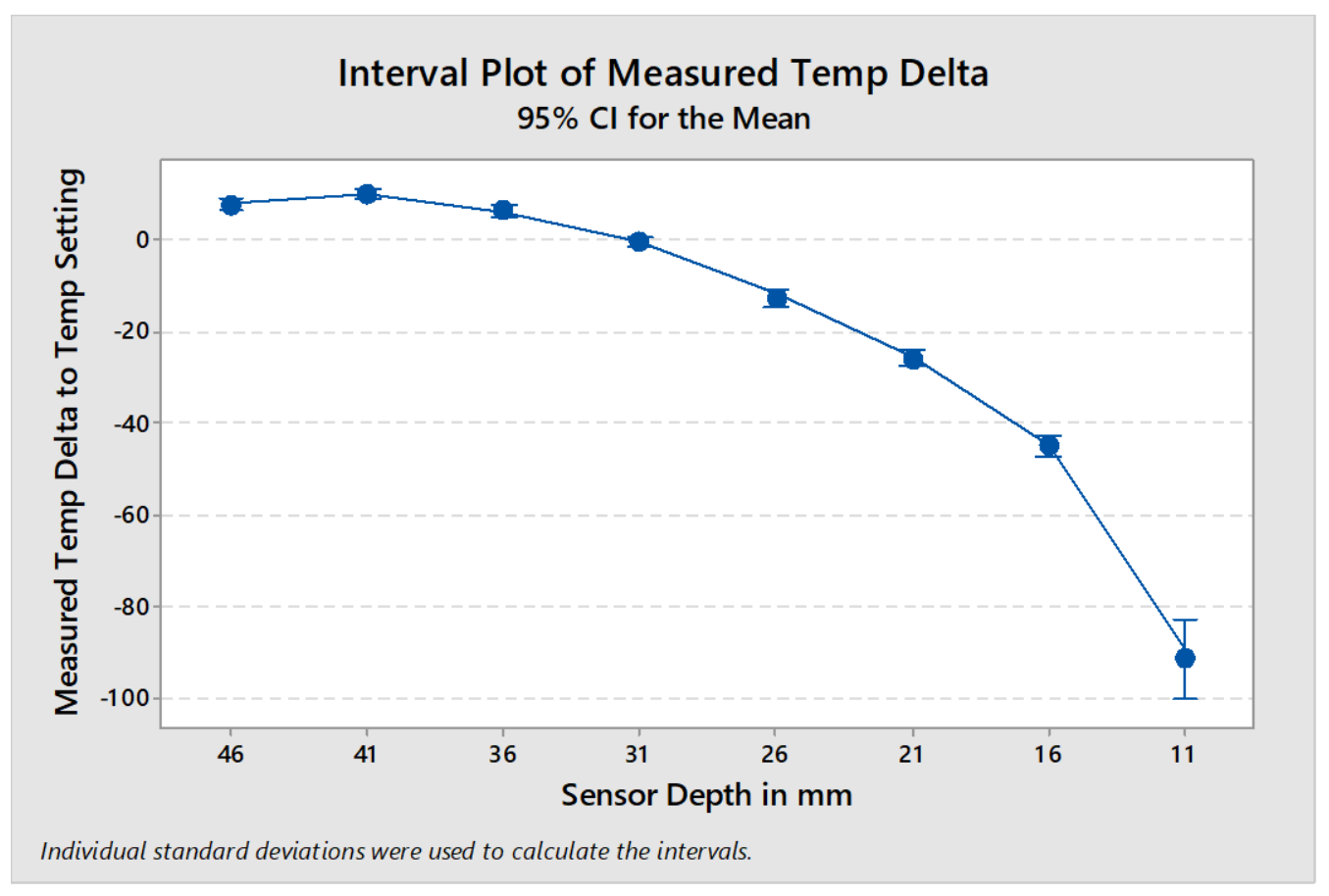

Figure 86 - Interval Plot for temperature delta from temperature setting

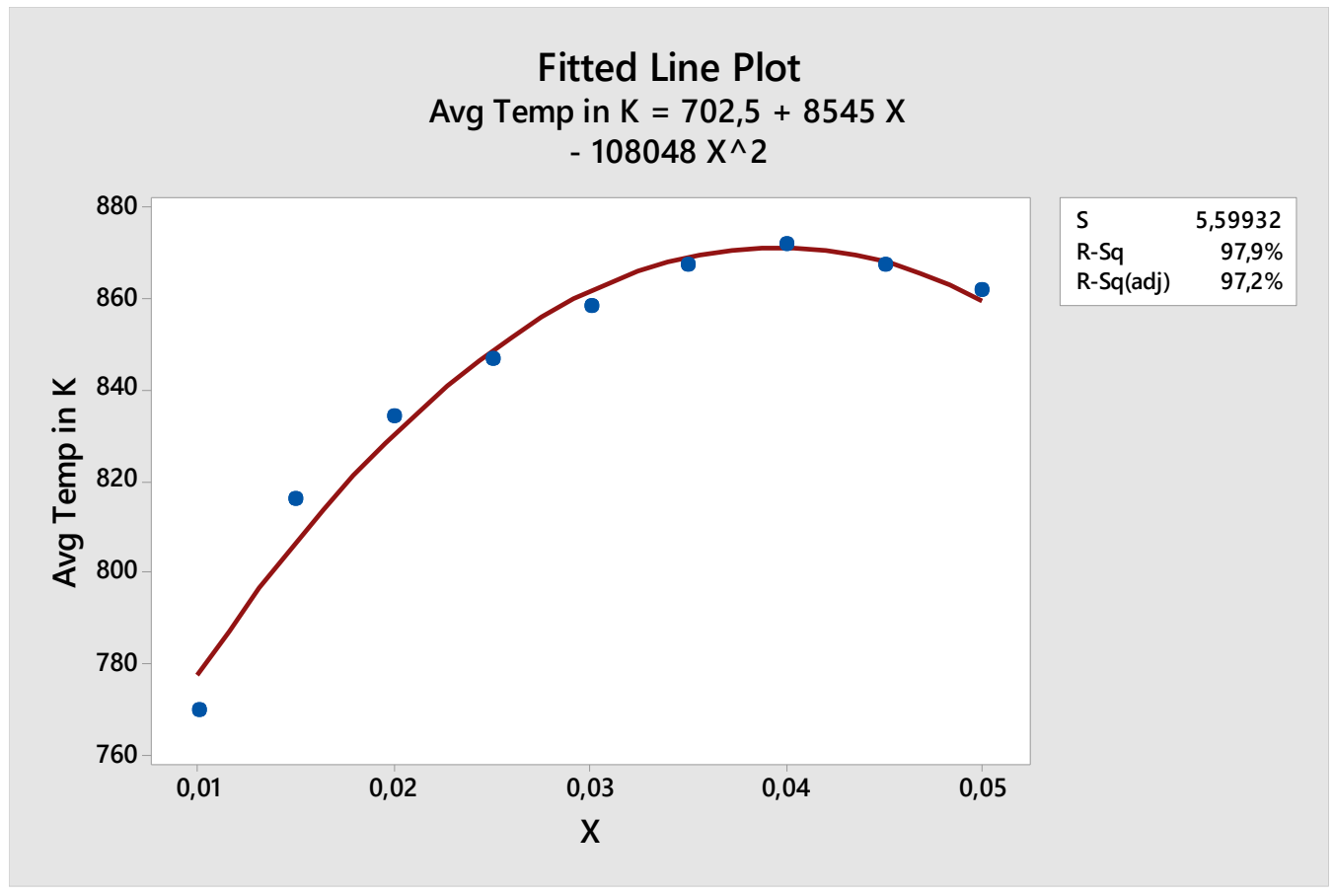

Figure 87 - Fitted line plot and temperature distribution function for $585^{\circ} \mathrm{C} / 858 \mathrm{~K}$ temperature setting 


\subsubsection{Adapted temperature distribution within the nozzle}

The adapted nozzle temperature function was added as a User Defined Function (UDF) into ANSYS Fluent (see Figure 88). In addition to the adapted temperature profile, the convection at the outlet has been taken into account assuming ambient temperature.

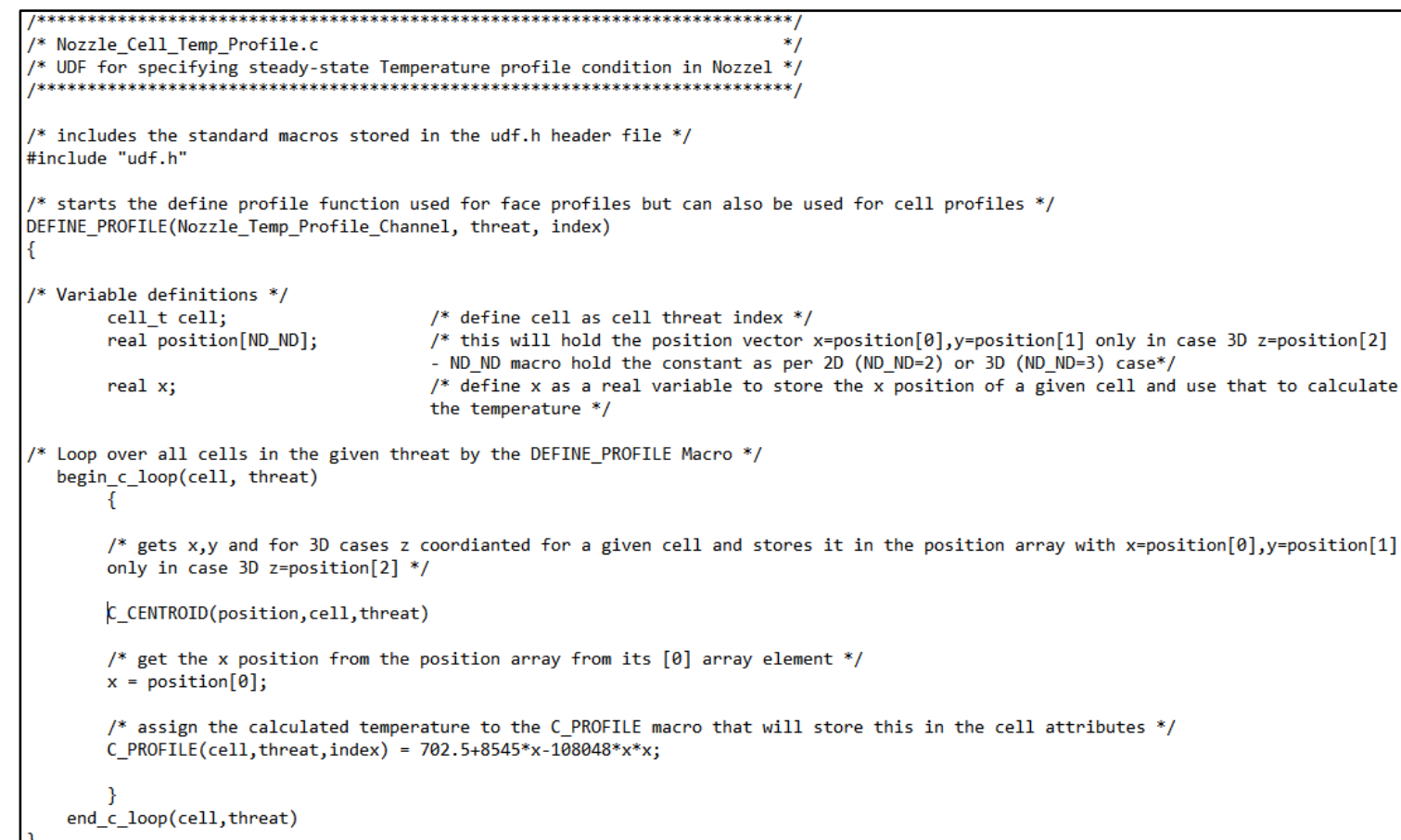

Figure 88 - ANSYS Fluent User defined function to apply the nozzle temperature distribution The results of the simulations (see Figure 89) showed a significant deviation from the initial uniform results (see Figure 85) and are aligned with observations during the proof of concept and experiments. 

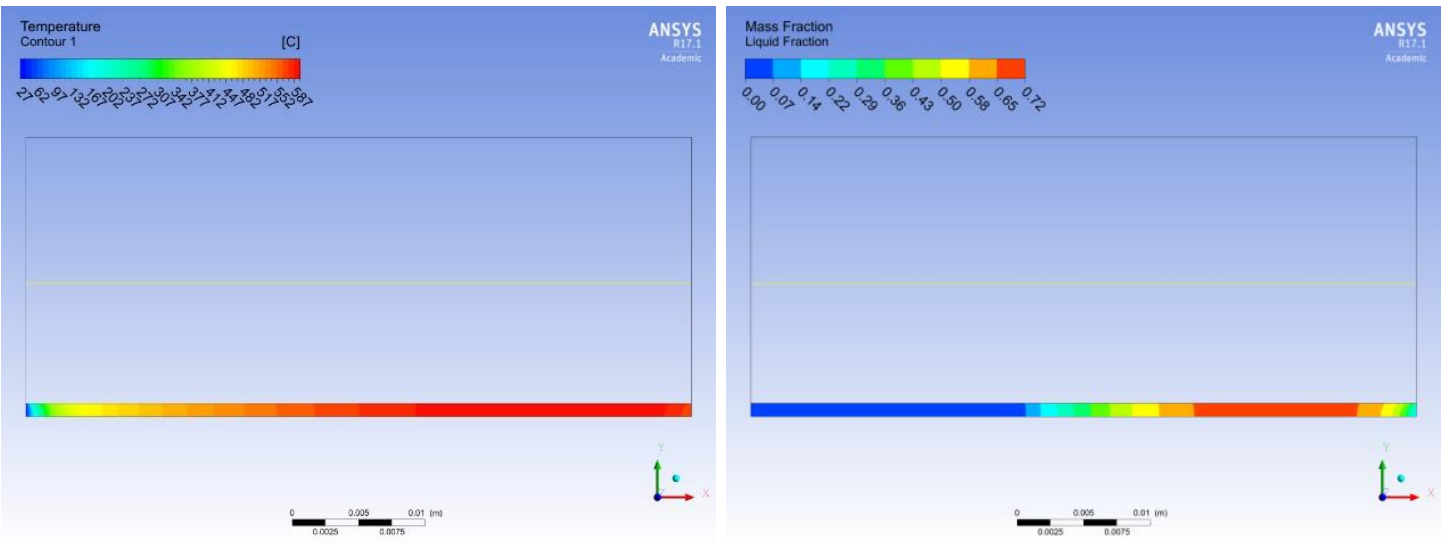

Figure 89 - Adapted Simulation Results for A356 heating with nozzle temperature profile Contour for resulting temperature and mass fraction liquid with setting $585^{\circ} \mathrm{C} / 858 \mathrm{~K}$

\subsubsection{Simulation of inductive heating}

To compare the induction heating with the resistive heating and to understand the potential application in a MEAM machine a simulation has been conducted. First preparation for a simulation is to understand the temperature dependent electrical resistivity / conductivity as shown in Figure 90. While Garcia [114] in his induction heating MEAM approach did not deem this as significant i.e. neglected that, it is strongly recommended by ANSYS to include the temperature dependency of the electrical conductivity.

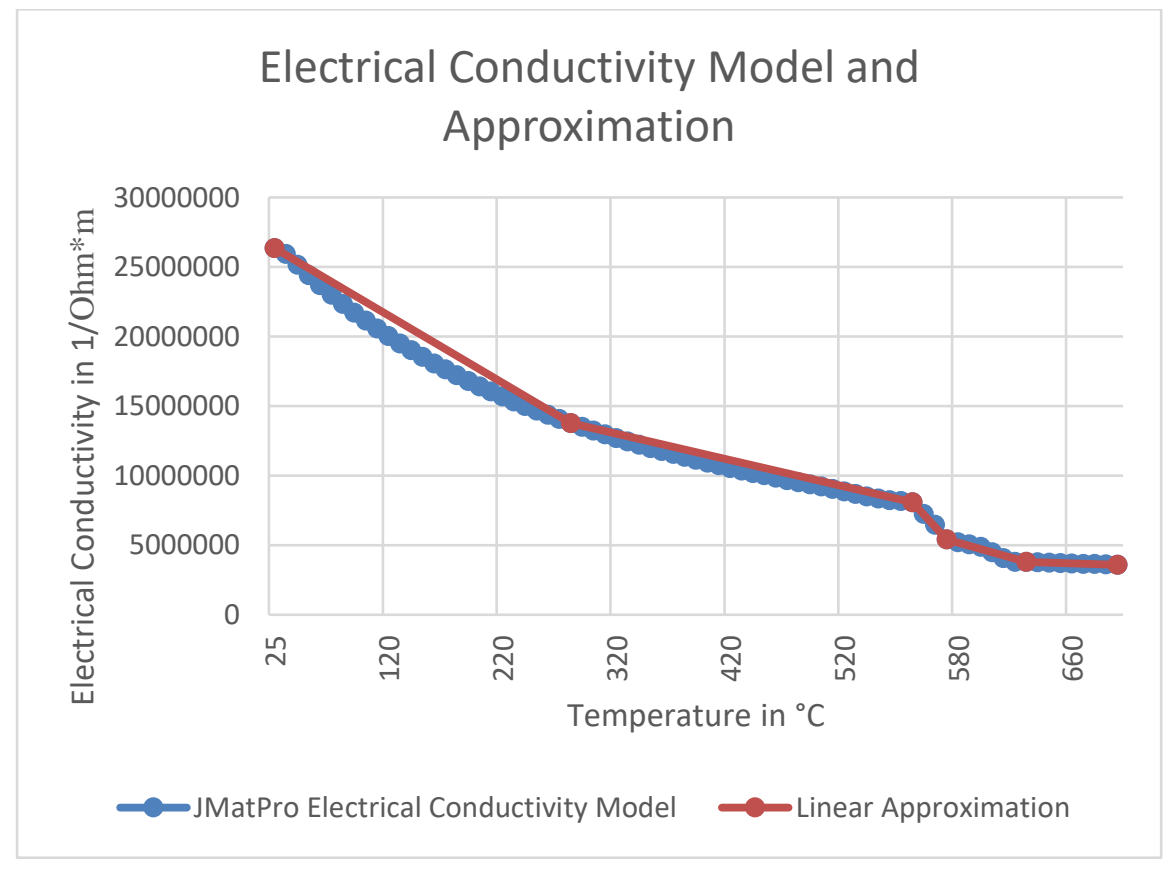

Figure 90 - A356 Electrical Conductivity / Temperature model and approximation 
The next preparation step for the induction heating simulation is to identify a meaningful frequency $(f)$ to be applied throughout the temperature range. Key influencing factors (see also equation 2.35) are the adequate skin depth $(\delta)$ for a thorough through heating and the electrical resistivity $(\sigma)$. As aluminum is a nonferrous metal the relative permeability $\left(\mu_{r}\right)$ is 1.00002 i.e. can be neglected and only the magnetic constant $\left(\mu_{0}=1.25664 \mathrm{E}-06\right)$ becomes relevant.

$$
f=\frac{\sigma}{\pi * \delta^{2} * \mu_{r} * \mu_{0}}
$$

Using the formula 4.2 the frequency can be calculated for various skin depths around $1 / 4$ of the filament diameter as referenced by Haimbaugh [120]. Table 14 shows the resulting frequencies for different skin depth and temperature.

Table 14 - Frequency calculation based on skin depth and temperature

\begin{tabular}{|c|c|c|c|}
\hline $\begin{array}{c}\text { Skin Depth } \\
\text { in } \mathbf{~}\end{array}$ & $\begin{array}{c}\text { Temperature } \\
\text { in }{ }^{\circ} \mathbf{C}\end{array}$ & $\begin{array}{c}\text { Electrical resistivity } \\
\text { in (Ohm* } \mathbf{m})\end{array}$ & $\begin{array}{c}\text { Frequency } \\
\text { in } \mathbf{~ k H z}\end{array}$ \\
\hline 0.0005 & 25 & $3.98005 \mathrm{E}-08$ & 40 \\
\hline 0.0005 & 555 & $1.1981 \mathrm{E}-07$ & 121 \\
\hline 0.0005 & 585 & $1.9898 \mathrm{E}-07$ & 202 \\
\hline 0.0005 & 620 & $2.6363 \mathrm{E}-07$ & 267 \\
\hline 0.0005 & 700 & $2.7851 \mathrm{E}-07$ & 282 \\
\hline 0.00033 & 25 & $3.98005 \mathrm{E}-08$ & 93 \\
\hline 0.00033 & 555 & $1.1981 \mathrm{E}-07$ & 279 \\
\hline 0.00033 & 585 & $1.9898 \mathrm{E}-07$ & 463 \\
\hline 0.00033 & 620 & $2.6363 \mathrm{E}-07$ & 613 \\
\hline 0.00033 & 700 & $2.7851 \mathrm{E}-07$ & 648 \\
\hline 0.00067 & 25 & $3.98005 \mathrm{E}-08$ & 11 \\
\hline 0.00067 & 555 & $1.1981 \mathrm{E}-07$ & 23 \\
\hline 0.00067 & 585 & $1.9898 \mathrm{E}-07$ & 28 \\
\hline 0.00067 & 620 & $2.6363 \mathrm{E}-07$ & 30 \\
\hline 0.00067 & 700 & $2.7851 \mathrm{E}-07$ & 26 \\
\hline
\end{tabular}

To allow an efficient heating at the target temperature (see Table 14) the frequency has been chosen as $200 \mathrm{kHz}$ for the Simulation.

A design with ANSYS Maxwell for the eddy current simulation and ANSYS Fluent has been created as seen in Figure 91. For the geometry a basic heating channel 
has been drawn with the filament inside and a 10 turn inductive work coil surrounding the channel (see Figure 92a). From that a 2D symmetric cross section has been created and used for the Maxwell and ANSYS simulation. For the Maxwell materials glass as an example nozzle material has been used and a filament material designed (referencing A356) using the temperature dependent approximation for the electrical conductivity/resistivity (see Figure 90).

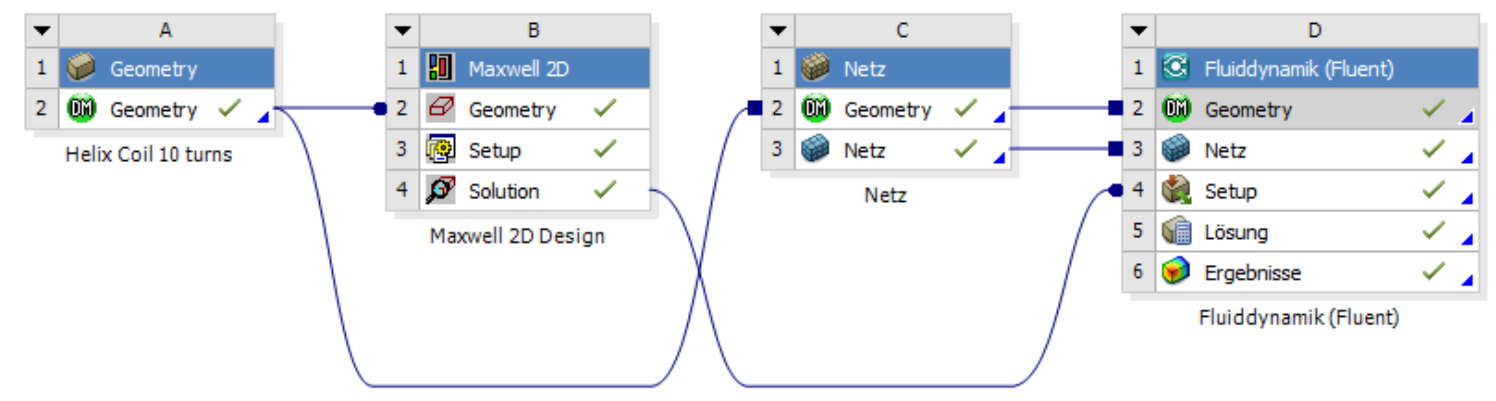

Figure 91 - Ansys induction heating simulation setup with Maxwell and Fluent

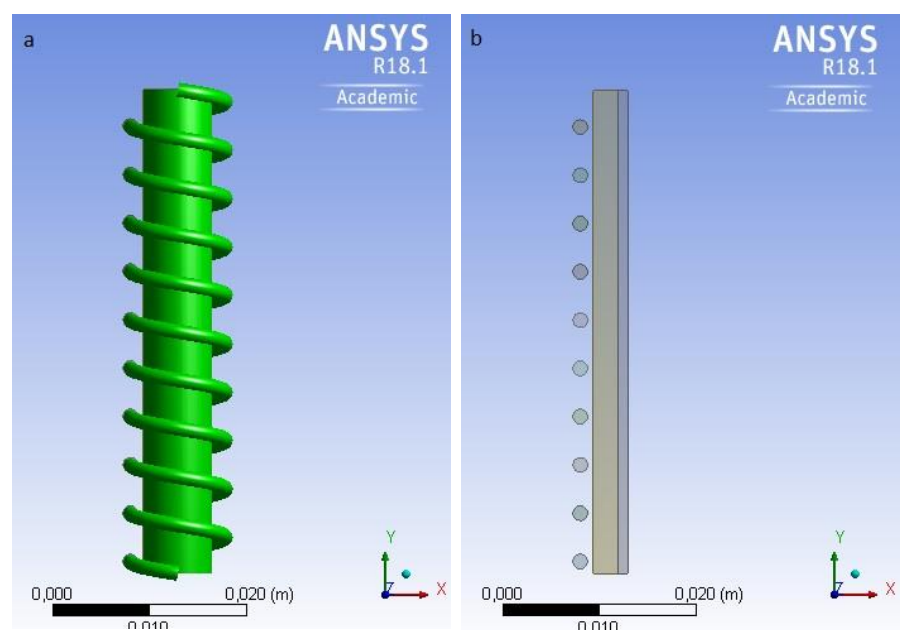

Figure 92 - Induction heating simulation geometry design a) 3D model b) 2D symetric cross section of 3D model

During initial simulations the ampere excitation for about $50 \%$ liquid fraction has been determined and simulations been run to understand the sensitivity of the work coil excitation vs. the liquid fraction. Each simulation requires multiple runs of Maxwell and Fluent as the feedback between both parts of the simulation is manual. All Fluent simulations have been done with a filament / inlet velocity of $0.005 \mathrm{~m} / \mathrm{s}$ as a low speed / controlled extrusion. 
Table 15 - Induction heating simulation run results for excitation of 315 Ampere

\begin{tabular}{|c|c|c|c|c|}
\hline Run & $\begin{array}{c}\text { Ohmic Losses } \\
\text { in W }\end{array}$ & $\begin{array}{c}\text { Max Temperature } \\
\text { in }{ }^{\circ} \mathbf{C}\end{array}$ & $\begin{array}{c}\text { Max Fraction Liquid } \\
\text { in \% }\end{array}$ & $\begin{array}{c}\text { Max delta T } \\
\text { in }{ }^{\circ} \mathbf{C}\end{array}$ \\
\hline 1 & 12260 & 560 & 7.8 & - \\
\hline 2 & 18691 & 573 & 30.5 & 538 \\
\hline 3 & 20009 & 581 & 42.5 & 79 \\
\hline 4 & 20292 & 582 & 45.3 & 12 \\
\hline 5 & 20346 & 583 & 46.1 & 1.9 \\
\hline 6 & 20361 & 583 & $\mathbf{4 6 . 2}$ & 0.4 \\
\hline
\end{tabular}

Table 16 - Induction heating simulation run results for excitation of 318 Ampere

\begin{tabular}{|c|c|c|c|c|} 
Run & $\begin{array}{c}\text { Ohmic Losses } \\
\text { in W }\end{array}$ & $\begin{array}{c}\text { Max Temperature } \\
\text { in }{ }^{\circ} \mathbf{C}\end{array}$ & $\begin{array}{c}\text { Max Fraction Liquid } \\
\text { in \% }\end{array}$ & $\begin{array}{c}\text { Max delta T } \\
\text { in }{ }^{\circ} \mathbf{C}\end{array}$ \\
\hline 1 & 12495 & 560 & 8.7 & - \\
\hline 2 & 19116 & 575 & 33.1 & 538 \\
\hline 3 & 20490 & 583 & 47.9 & 78.9 \\
\hline 4 & 20821 & 585 & 50.6 & 12.6 \\
\hline 5 & 20882 & 586 & 51.6 & 1.7 \\
\hline 6 & 20898 & 586 & $\mathbf{5 2 . 2}$ & 0.7 \\
\hline
\end{tabular}

Table 17- Induction heating simulation run results for excitation of 321 Ampere

\begin{tabular}{|c|c|c|c|c|} 
Run & $\begin{array}{c}\text { Ohmic Losses } \\
\text { in W }\end{array}$ & $\begin{array}{c}\text { Max Temperature } \\
\text { in }{ }^{\circ} \mathbf{C}\end{array}$ & $\begin{array}{c}\text { Max Fraction Liquid } \\
\text { in \% }\end{array}$ & $\begin{array}{c}\text { Max delta T } \\
\text { in }{ }^{\circ} \mathbf{C}\end{array}$ \\
\hline 1 & 12732 & 561 & 9.6 & - \\
\hline 2 & 19546 & 578 & 37.3 & 539 \\
\hline 3 & 20982 & 587 & 52.5 & 78.5 \\
\hline 4 & 21374 & 589 & 56.3 & 12.4 \\
\hline 5 & 21450 & 593 & 63.1 & 2.3 \\
\hline 6 & 21455 & 589 & 57.2 & 3.5 \\
\hline 7 & 21451 & 589 & 56.7 & 3.1 \\
\hline 8 & 21451 & 593 & 63.1 & 0.9 \\
\hline
\end{tabular}

Figure 93 shows the resulting ohmic losses in Maxwell after one run in the eddy current simulation including the flux lines (left) and after all Maxwell - Fluent temperature feedback loops (right). After the initial eddy current simulation, the filament shows nearly the same ohmic losses from the Inlet (top) to the outlet bottom. After all temperature feedback loops the ohmic losses increases due to 
the stable frequency $(200 \mathrm{kHz})$ but larger dependent penetration depth at higher temperature. After the first run the maximum liquid mass fraction is in each excitation cases (315A, 318A, 321A) below $10 \%$. This shows the significance of the temperature dependent electrical conductivity for the simulation.

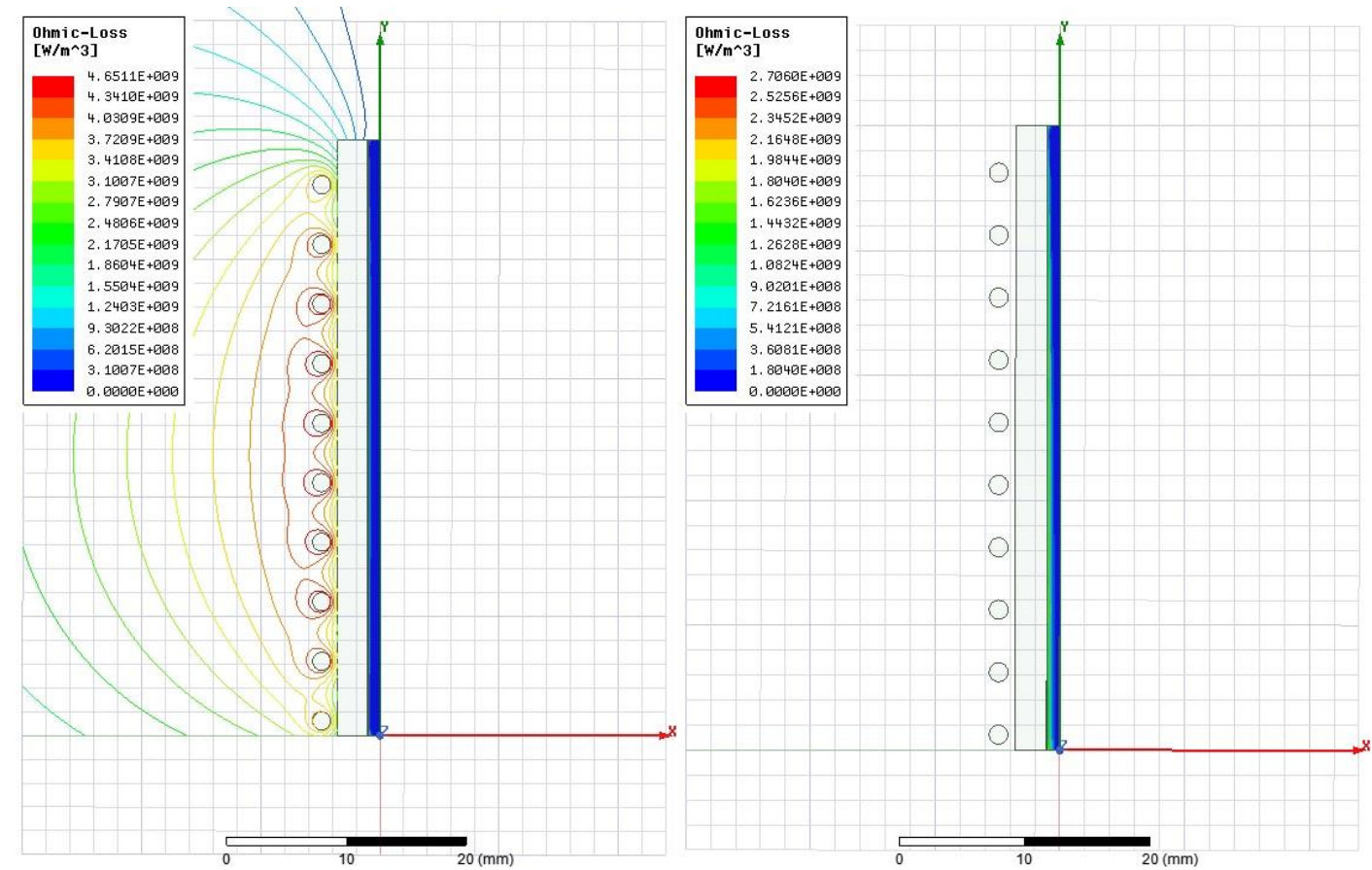

Figure 93 - Ohmic Losses - left) after one simulation pass right) after last simulation pass

\subsection{Experiments}

\subsubsection{Heating to globular microstructure experiment}

Based on current microstructure condition of the filament (see section 3.3), the simulation results (see section 5.4) and nozzle temperature distribution (see section 4.1.2.2) an experiment has been designed to identify an adequate process for globular microstructure preparation ready to extrude in a semi-solid state. The goal is to choose the adequate parameter for a following extrusion experiment as well as understand the parameter - response relationships. For that purpose, the Rheocast Quality Index (RQI - see section 2.1.10 and equation 2.20) was chosen as the metric. Besides the RQI it is important to also observe grain size and if possible verify the fraction liquid. 
To achieve a relative uniform temperature for the heated alloy a $2 \mathrm{~cm}$ sample filament size was chosen at the most temperature-stable area (Placement depth: $2 \mathrm{~cm}-4 \mathrm{~cm}$ ) of a nozzle without a neck. For that the replaceable nozzle tip was removed (see Figure 94 left).

To reduce the adhesion of the semi-solid material with the nozzle wall Boron Nitride ceramic spray (see Figure 94 right) has been applied to the nozzle and the filament ${ }^{23}$.
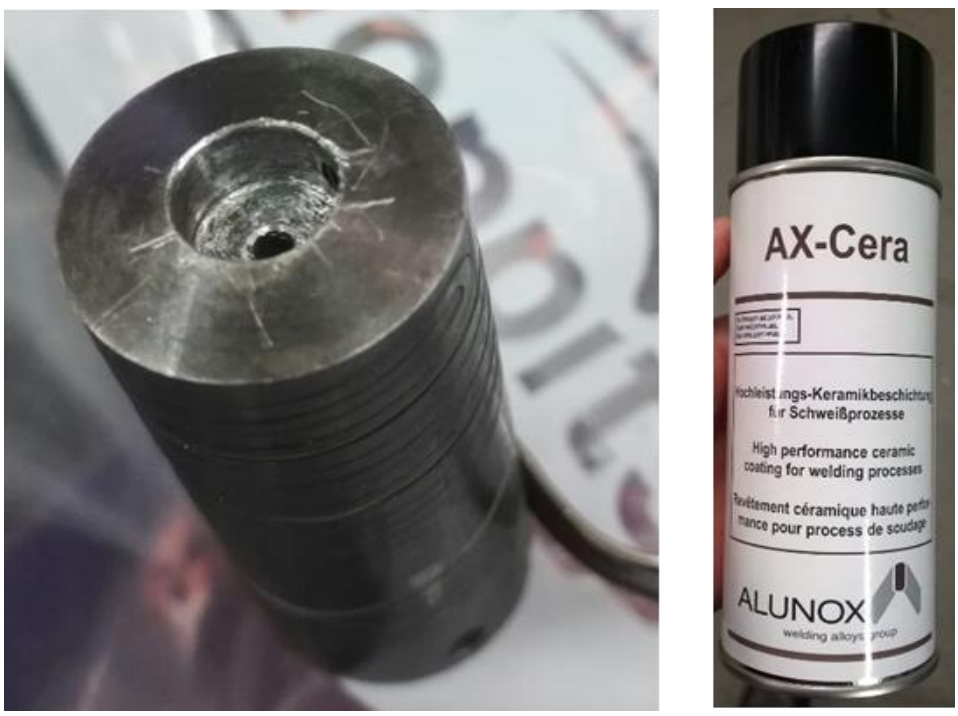

Figure 94 - left) Nozzle without tip right) Boron Nitride ceramic spray

The heating as well as following experiments were performed using metal pins (see Figure 95 left for a pin in the test tube) as a piston to push the sample through the channel or later through the nozzle neck. A test tube has been used to quench the sample as well as keep the extruded sample oriented for marking. The following setup and execution process was used for each sample:

1. Review clean and coated nozzle

2. Prepare $2 \mathrm{~cm}$ filament (A356 or THIXALLOY 540) with Boron Nitride

3. Heating nozzle to the temperature range and wait $5 \mathrm{~min}$

\footnotetext{
${ }^{23}$ Note: this is not an adequate approach for future layer bonding as the ceramic coating of the filament would restrict the bonding to the next layer. It has been applied on both nozzle and filament to ensure a more effective coating. A future MEAM nozzle would be produced using coating processes such as Chemical Vapor Deposition. This would be applied to the nozzle only.
} 
4. Find setting temperature for target temperature $+2^{\circ} \mathrm{C}$ at $3.5 \mathrm{~cm}$

5. Insert Filament $2 \mathrm{~cm}$ deep into the heating channel

6. After target time passed press sample into the test tube with water

7. Mark the sample upper area and document

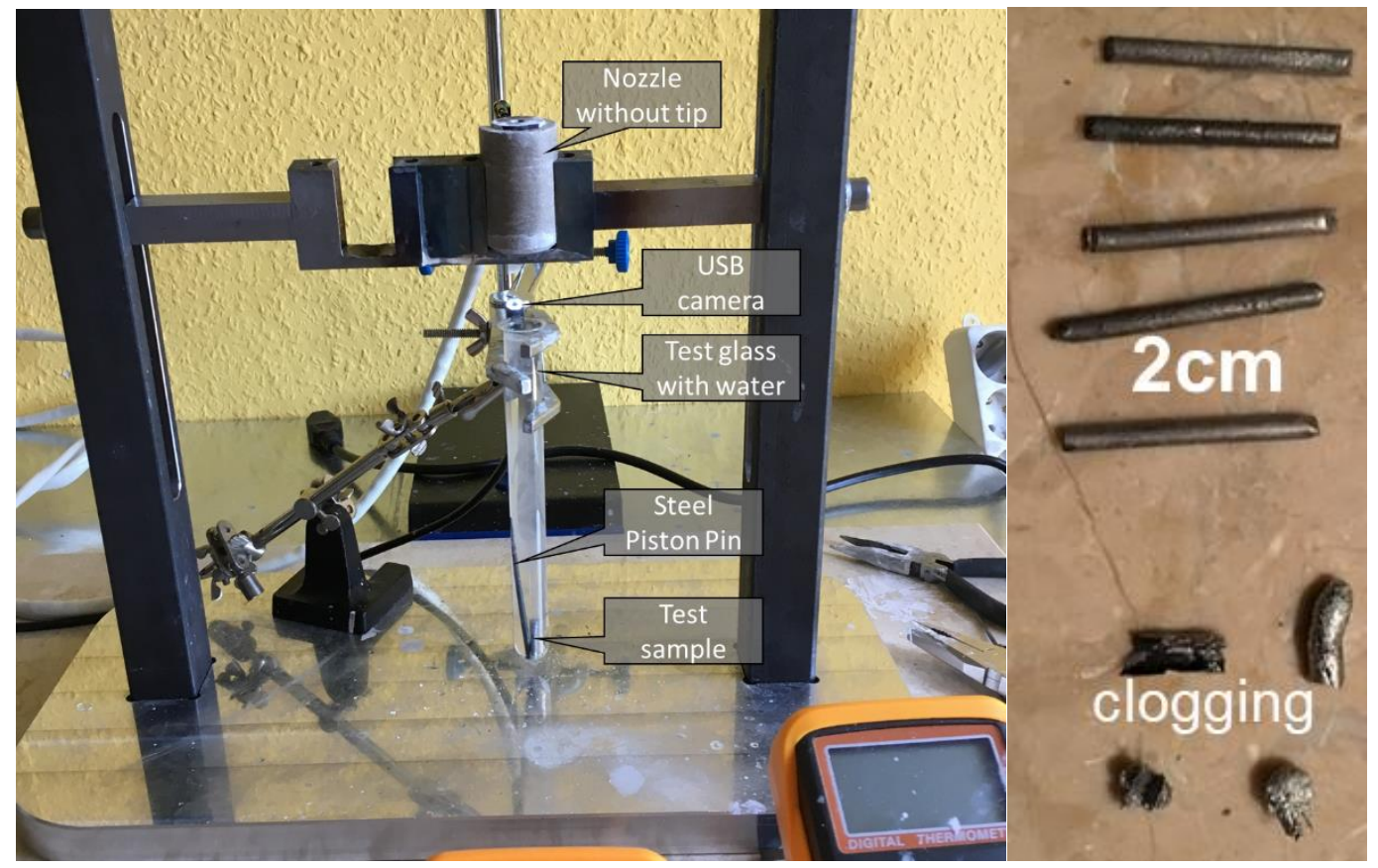

Figure 95 - Left) Experiment Setup right) sample results

The experiment execution was designed using DOE. For that the initial heating experiment was used to identify the standard deviation.

Table 18 - Initial heating experiment results

\begin{tabular}{|c|c|c|}
\hline Temperature in ${ }^{\circ} \mathrm{C}$ & Run & Average Diameter in $\mu \mathrm{m}$ \\
\hline 575 & 1 & 45.63 \\
\hline 575 & 2 & 44.869 \\
\hline 575 & 3 & 43.788 \\
\hline 575 & 4 & 44.465 \\
\hline 575 & 5 & 44.404 \\
\hline 580 & 1 & 47.833 \\
\hline 580 & 2 & 47.575 \\
\hline
\end{tabular}




\begin{tabular}{|c|c|c|}
\hline Temperature in ${ }^{\circ} \mathrm{C}$ & Run & Average Diameter in $\mu \mathrm{m}$ \\
\hline 580 & 3 & 49.082 \\
\hline 580 & 4 & 47.673 \\
\hline 580 & 5 & 48.343 \\
\hline 585 & 1 & 49.614 \\
\hline 585 & 2 & 47.751 \\
\hline 585 & 3 & 47.754 \\
\hline 585 & 4 & 49.213 \\
\hline 585 & 5 & 48.229 \\
\hline 590 & 1 & 50.598 \\
\hline 590 & 2 & 50.909 \\
\hline 590 & 3 & 47.225 \\
\hline 590 & 4 & 49.008 \\
\hline 590 & 5 & 49.475 \\
\hline
\end{tabular}

$\begin{array}{lrrrrr}\text { Analysis of Variance } & \\ \text { Source } & \text { DF } & \text { Adj SS } & \text { Adj MS } & \text { F-Value } & \text { P-Value } \\ \quad \text { Temp } & 3 & 66.37 & 22.1218 & 23.71 & 0.000 \\ \text { Error } & 16 & 14.93 & 0.9331 & & \\ \text { Total } & 19 & 81.29 & & & \end{array}$

Calculation of Standard Deviation:

MSE $=0.9331 \quad \sigma=\sqrt{M S_{E}}=0.966$

Based on the calculated standard deviation a full factorial design was created using Minitab for each alloy (A356 and THIXALLOY540):

Power and Sample Size

General Full Factorial Design

$\alpha=0.05$ Assumed standard deviation $=0.966$

Factors: 2 Number of levels: $3 ; 2$

Include terms in the model up through order: 2

Not including blocks in model. 


$\begin{array}{rrrrr}\text { Maximum } & & \text { Total } & \text { Target } & \\ \text { Difference } & \text { Reps } & \text { Runs } & \text { Power } & \text { Actual Power } \\ 1 & 15 & 90 & 0.95 & 0.950794 \\ 1 & 13 & 78 & 0.90 & 0.915679 \\ 2 & 5 & 30 & 0.95 & 0.979531 \\ 2 & 4 & 24 & 0.90 & 0.935018 \\ 3 & 3 & 18 & 0.95 & 0.991592\end{array}$

With the design above, 4 replicates to detect a difference of $2 \mu \mathrm{m}$ where chosen expecting an actual power of 0.935 (see also Figure 96). This was deemed sufficient as the microstructure analysis and further processing is at a significant cost.

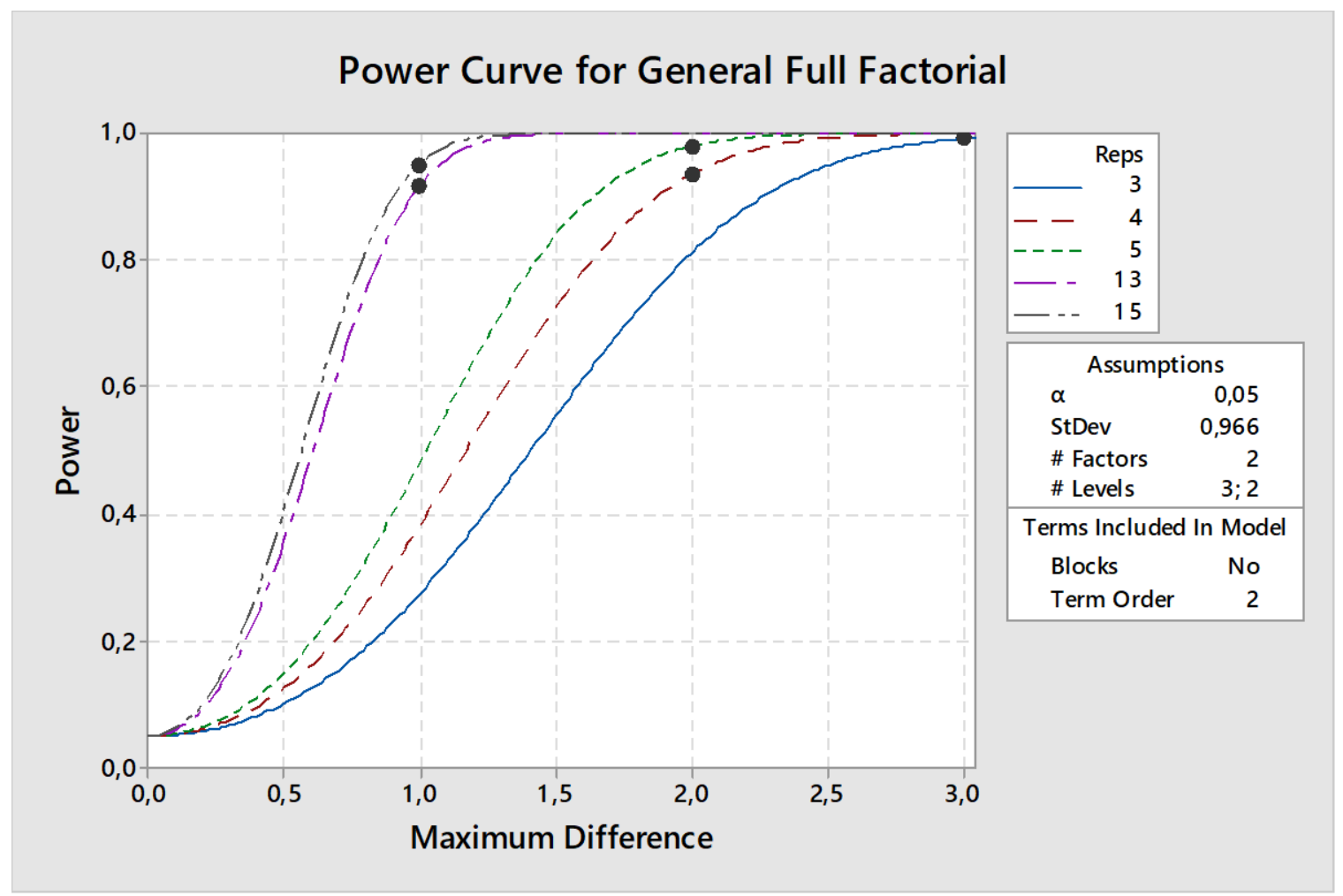

Figure 96 - Power Curve for full factorial design for the heating experiment

The full factorial randomized design is shown in Appendix 0.

After the first results reviewed it was identified that the lower temperature value for THIXALLOY 540 was not chosen in a range that develops an adequate microstructure for analysis (see Figure 97). As this alloy is just a comparative one it was chosen to rely on the higher temperature only. Further on, due to clogging within the nozzle, 106 samples attempts were needed to achieve the expected remaining 40 samples. 


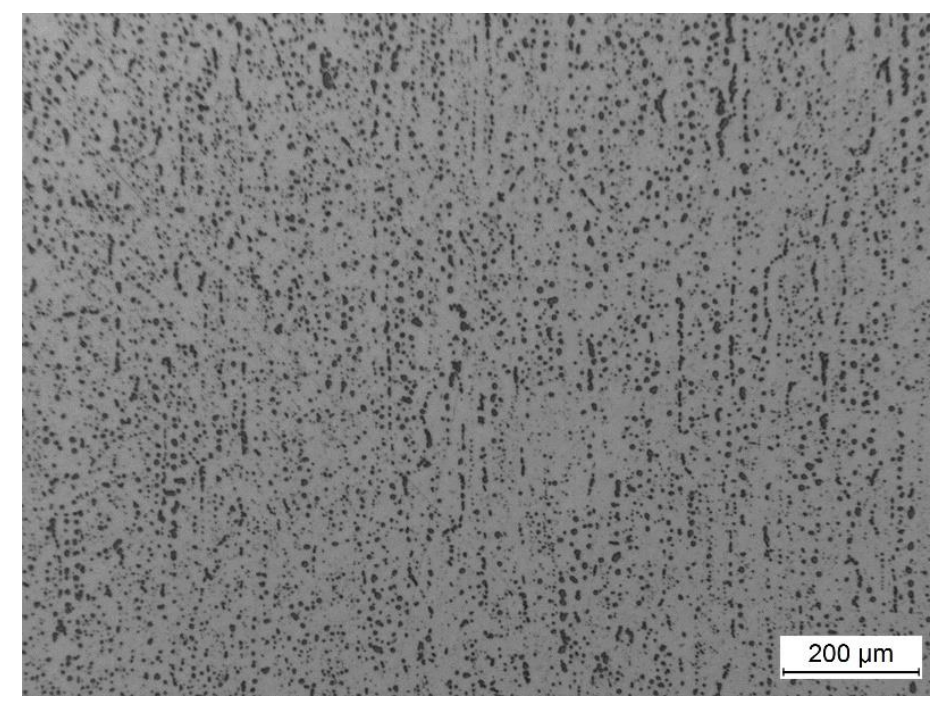

Figure 97 - Example of THIXALLOY 540 low temperature with inadequate microstructure

For each sample, microstructure analysis was prepared and micrographs where taken. Due to the length of the sample and measured temperature distribution in the nozzle a derived model for the 3 micrographs (upper, middle and lower area) has been created (see Table 19 and Table 20). This results in 120 micrographs for the 40 samples created.

Table 19 - Temperature model for A356 heating samples with marked overlaps

\begin{tabular}{|c|c|c|c|}
\hline $\begin{array}{c}\text { Temperature } \\
\text { setting at } \mathbf{3 . 5 \mathrm { cm }}\end{array}$ & $\begin{array}{c}\text { Temperature of } \\
\text { sample upper area }\end{array}$ & $\begin{array}{c}\text { Temperature of } \\
\text { sample middle } \\
\text { area }\end{array}$ & $\begin{array}{c}\text { Temperature of } \\
\text { sample lower area }\end{array}$ \\
\hline Normalized $\mathbf{0}^{\circ} \mathbf{C}$ & $-3^{\circ} \mathrm{C}$ & $0^{\circ} \mathrm{C}$ & $-12^{\circ} \mathrm{C}$ \\
\hline $\mathbf{5 8 5}^{\circ} \mathbf{C}$ (Low) & $582^{\circ} \mathrm{C}$ & $585^{\circ} \mathrm{C}$ & $573^{\circ} \mathrm{C}$ \\
\hline $5^{\circ} 5^{\circ} \mathbf{C}$ (High) & $592^{\circ} \mathrm{C}$ & $595^{\circ} \mathrm{C}$ & $583^{\circ} \mathrm{C}$ \\
\hline
\end{tabular}

Note the highlighted High temperature/lower area and Low temperature/upper area temperature are just $1^{\circ} \mathrm{C}$ different within the model. It should be tested whether the results do not significantly differ from each other. This will also allow to validate the model if in other cases the temperature would show significant difference. 
Table 20 - Temperature model for THIXALLOY 540 heating samples

\begin{tabular}{|c|c|c|c|}
\hline $\begin{array}{c}\text { Temperature } \\
\text { setting at } 3.5 \mathrm{~cm}\end{array}$ & $\begin{array}{c}\text { Temperature of } \\
\text { sample upper area }\end{array}$ & $\begin{array}{c}\text { Temperature of } \\
\text { sample middle } \\
\text { area }\end{array}$ & $\begin{array}{c}\text { Temperature of } \\
\text { sample lower area }\end{array}$ \\
\hline Normalized $\mathbf{0}^{\circ} \mathbf{C}$ & $-3^{\circ} \mathrm{C}$ & $0^{\circ} \mathrm{C}$ & $-12^{\circ} \mathrm{C}$ \\
\hline $\mathbf{6 0 5}^{\circ} \mathbf{C}$ (High) & $602^{\circ} \mathrm{C}$ & $605^{\circ} \mathrm{C}$ & $593^{\circ} \mathrm{C}$ \\
\hline
\end{tabular}

After micrographs are taken the following measurements need to be completed:

- Microstructure / Macrostructure correlation

- Perimeter and area of each full grain resulting in average values per micrograph

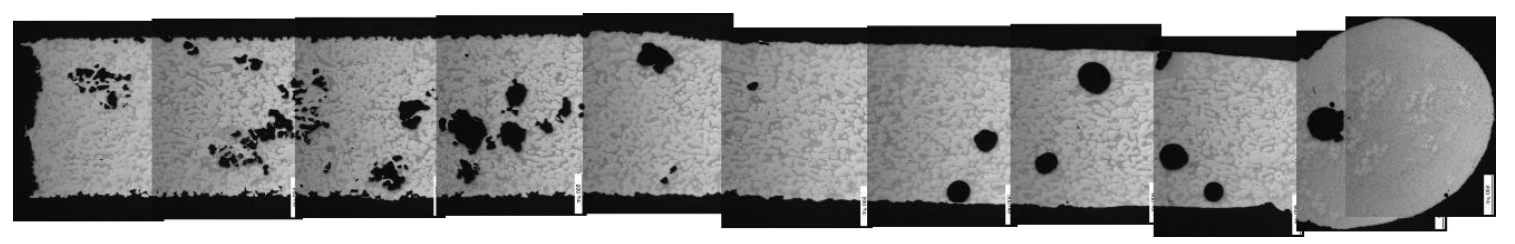

Figure 98 - Example panorama picture of Microstructure - Sample 18 - A356 15min @ 585 ${ }^{\circ} \mathrm{C}$

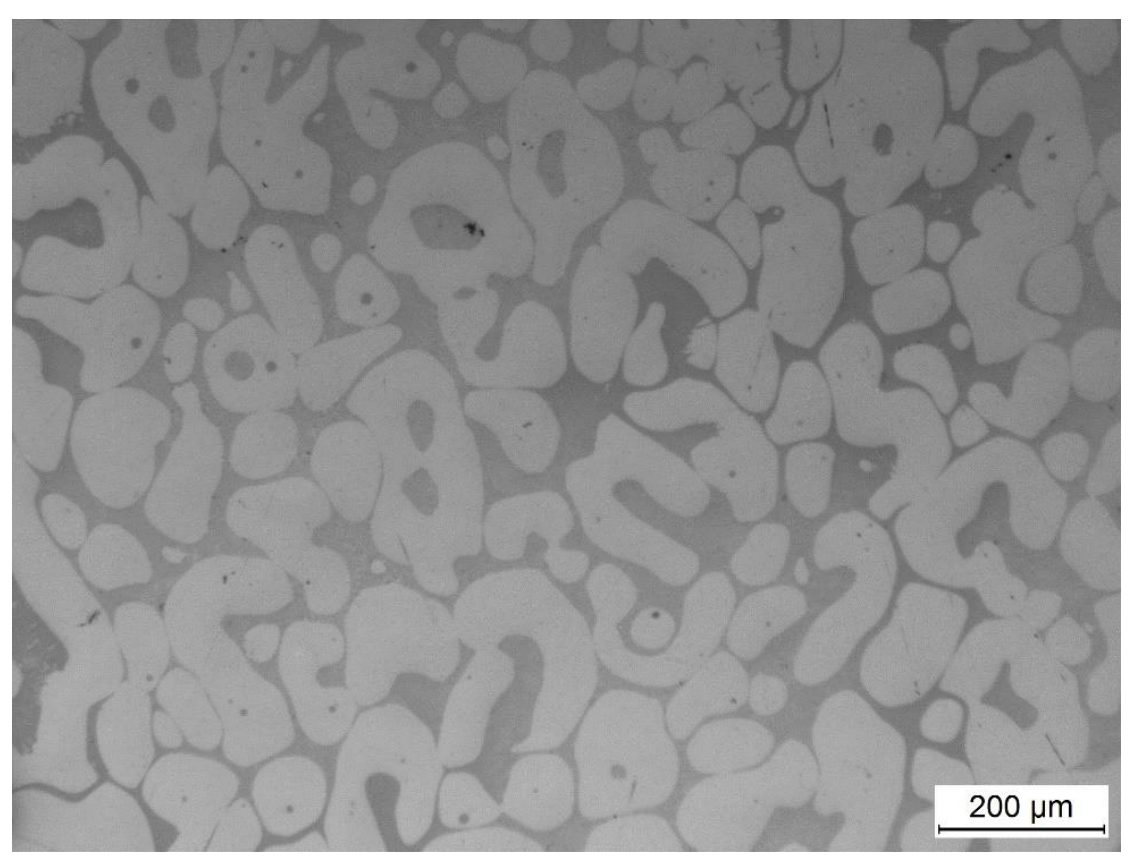

Figure 99 - Example of chosen micrograph for sample 18 middle at 50x maginification 
The Micro- and Macrostructure was counted using the method described by Zoqui [37] (see Figure 100). For each micrograph 5 vertical samples and 5 horizontal samples are taken (see Figure 101 left) and the average has been used for this sub-sample (see Figure 101 right).

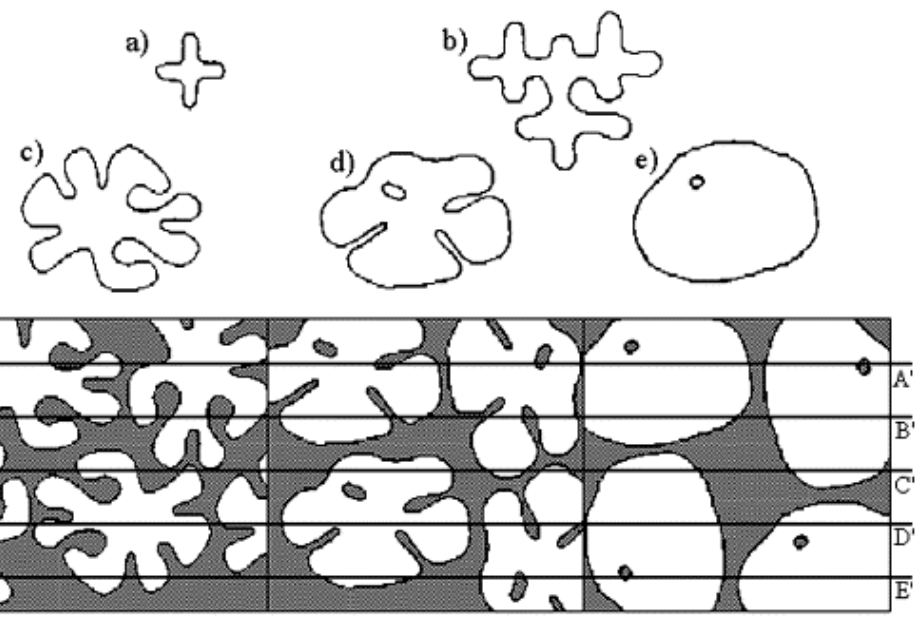

f)

g)

h)

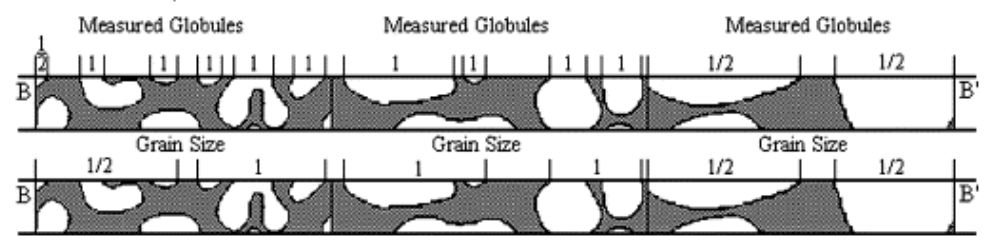

i)

J)

k)

Figure 100 - Macro- and microstructure analysis approach by Zoqui et.al. [37]

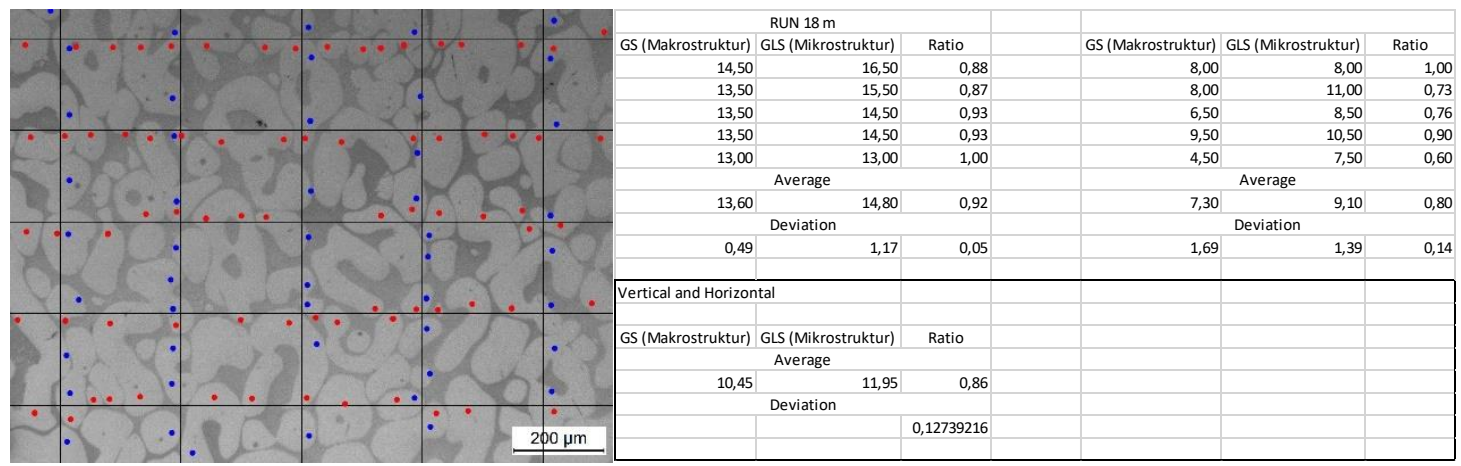

Figure 101 - left) sample micrograph - right) results of macro- and microstructure analysis

The Rheocast Quality Index (RQI) requires relatively precise measurement of perimeter and area of the grains. Therefore, the grains have not been automatically segmented as done prior in the initial filament review (see section 3.1). This had 
led to a higher spherical shape than observed in the sample. ImageJ and Gimp have been used to process the images through a multi-step approach as shown in Appendix 0 . The main process steps are smoothing, removal of scale, black/white transfer, removal of small particles (see Figure 102a), manual grain segmentation (Figure 102b), inverting (Figure 102c) and measuring (see Table 21 and Table 22).

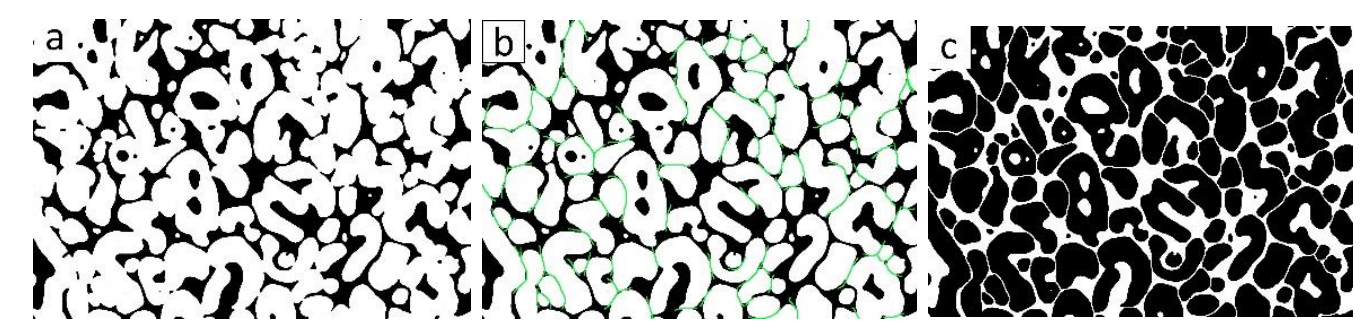

Figure 102 - Sample 18M image processing a) BW transfer \& smooth b) manual segmentation c) inverted final image for measurement

Eleven Micrographs are found where an analysis is not possible due to inadequate microstructure development. Those have been excluded from further review.

Table 21 - Example of individual grain results of sample 18

\begin{tabular}{|c|c|c|c|}
\hline Grain count & Area & Perim. & Circ. \\
\hline 1 & 160.387 & 46.565 & 0.93 \\
\hline 2 & 5056.275 & 274.596 & 0.843 \\
\hline 3 & 35771.029 & 1277.821 & 0.275 \\
\hline 4 & 3080.462 & 223.169 & 0.777 \\
\hline 5 & 130.718 & 42.631 & 0.904 \\
\hline 6 & 2988.874 & 220.003 & 0.776 \\
\hline 7 & 2535.232 & 202.197 & 0.779 \\
\hline 8 & 23103.897 & 687.298 & 0.615 \\
\hline 9 & 1243.967 & 132.53 & 0.89 \\
\hline 10 & 325.504 & 77.141 & 0.687 \\
\hline$\ldots$ & $\ldots$ & $\ldots$ & $\ldots$ \\
\hline 82 & 5478.957 & 287.525 & 0.833 \\
\hline
\end{tabular}


Table 22 - Area and perimeter average of Sample 18M

\begin{tabular}{|c|c|c|c|c|c|c|}
\hline Sample & Count & Total Area & $\begin{array}{c}\text { Average } \\
\text { Size }\end{array}$ & \%Area & $\begin{array}{c}\text { Average } \\
\text { Perimeter }\end{array}$ & Circularity \\
\hline $\begin{array}{c}18 \mathrm{M}-\mathrm{FS} \\
\text { without } \\
\text { entrapped FL }\end{array}$ & 119 & 898704.642 & 7552.14 & 72.895 & 359.735 & 0.661 \\
\hline $\begin{array}{c}18 \mathrm{M}-\mathrm{FS} \text { with } \\
\text { entrapped FL }\end{array}$ & 119 & 883092.07 & 7420.942 & 71.629 & 359.735 & 0.658 \\
\hline $\begin{array}{c}18 \mathrm{M}-\text { border } \\
\text { grains } \\
\text { removed }\end{array}$ & 82 & 618622.513 & 7544.177 & 50.177 & 353.874 & 0.704 \\
\hline
\end{tabular}

The above results have been used to calculate the RQI as shown in Table 23 . See Appendix 0 and 0 for all A356 and THIXALLOY 540 results.

Table 23 - RQI calculation example for sample 18

\begin{tabular}{|c|c|c|c|c|c|c|c|c|}
\hline Run & $\begin{array}{c}\text { Actual } \\
\text { Temp }\end{array}$ & Area & Perimeter & $\begin{array}{c}\text { Shape } \\
\text { Factor }\end{array}$ & Diameter & GS & GLS & RQI \\
\hline 18 & 573 & 9494.525 & 429.110 & 1.54 & 88.50 & 10.25 & 11.25 & $\mathbf{0 . 5 9 0}$ \\
\hline 18 & 585 & 7544.177 & 353.874 & 1.32 & 85.28 & 10.45 & 11.95 & $\mathbf{0 . 6 6 2}$ \\
\hline 18 & 582 & 10513.767 & 442.178 & 1.48 & 95.11 & 9.60 & 11.50 & $\mathbf{0 . 5 6 4}$ \\
\hline
\end{tabular}

For the failed sample attempts it has been tested whether the Alloy, Temperature or holding time has a significant effect on the failure occurrence (see Appendix 0 ). There is now evidence of such effects and it is assumed that those failures are random. 


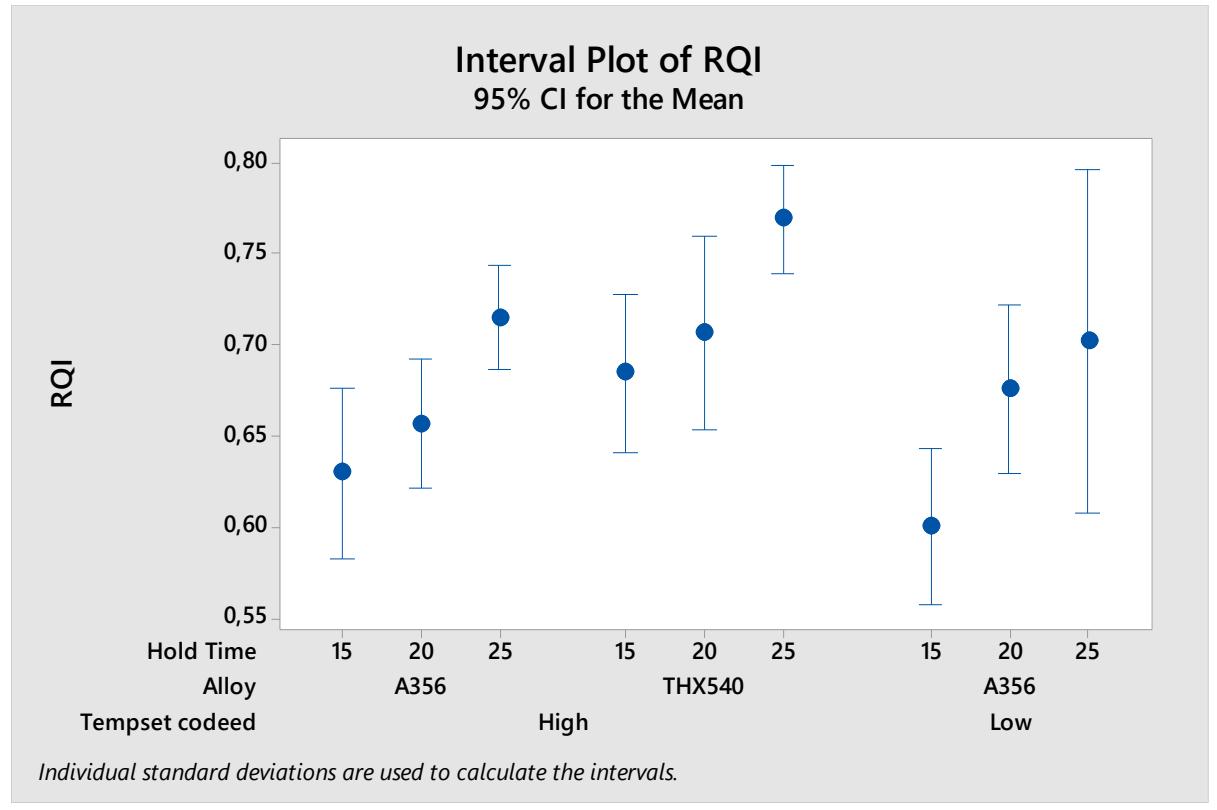

Figure 103 - Interval plot of RQI for temperature settings, alloy and hold Time

With the RQI results at hand the first review required is to verify the temperature model i.e. whether the A356 samples High Temperature / upper area vs. Low Temperature / lower area have a significant effect on the RQI. As the ANOVA below shows there is no statistical significance in those sample groups for this factor. As such the model is deemed appropriate.

\section{General Linear Model: RQI versus O-M-U; Hold Time Factor Information}

\begin{tabular}{llrl} 
Factor & Type & Levels & Values \\
\hline O-M-U & Fixed & 2 & O; U \\
Hold Time & Fixed & 3 & $15 ; 20 ; 25$
\end{tabular}

Analysis of Variance

\begin{tabular}{lrrrrr} 
Source & DF & Adj SS & Adj MS & F-Value & P-Value \\
\hline O-M-U & 1 & 0.000423 & 0.000423 & 0.08 & 0.781 \\
Hold Time & 2 & 0.068091 & 0.034046 & 6.45 & 0.009 \\
Error & 16 & 0.084511 & 0.005282 & & \\
Lack-of-Fit & 2 & 0.009889 & 0.004944 & 0.93 & 0.418 \\
Pure Error & 14 & 0.074622 & 0.005330 & & \\
Total & 19 & 0.158706 & & &
\end{tabular}

Model Summary 


\begin{tabular}{rrrr} 
S & R-sq & R-sq(adj) & R-sq(pred) \\
\hline 0.0726769 & $46.75 \%$ & $36.77 \%$ & $15.40 \%$
\end{tabular}

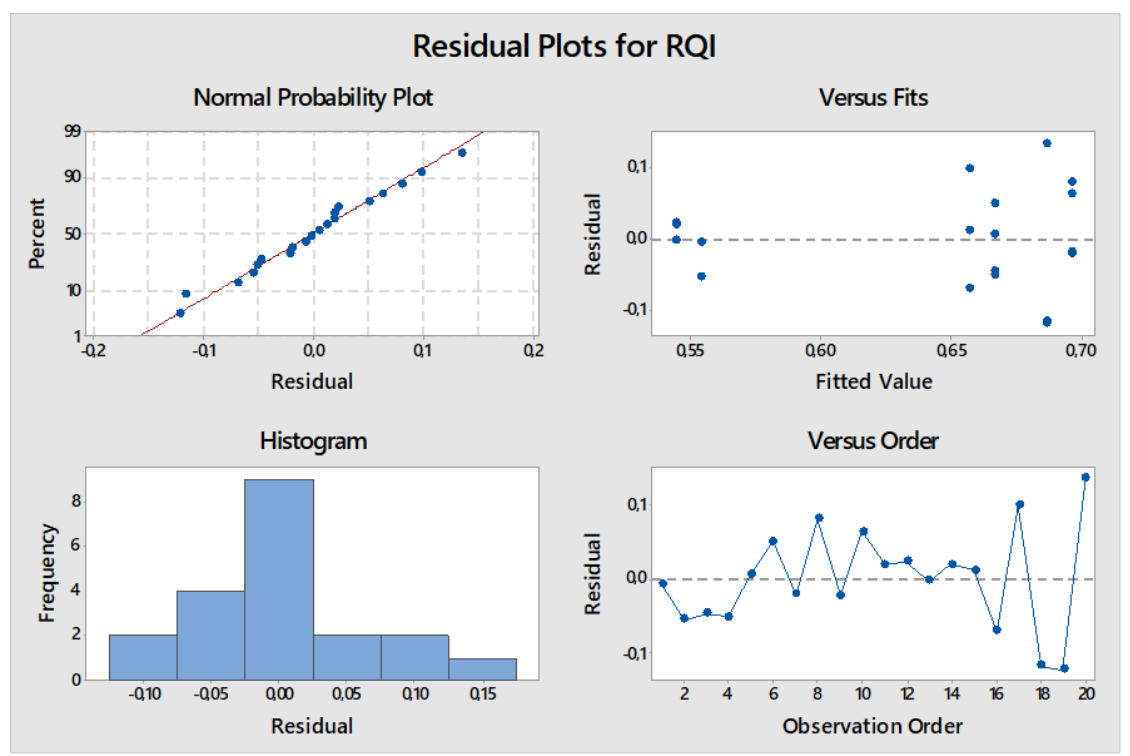

Figure 104 - Residuals Plot for the A356 Temperature Model verification

To understand the main effects and potential interactions the below ANOVA was reviewed. The results show no significant effect on the hold time and actual temperature interaction but a significant effect of the hold time and temperature independently.

\section{General Linear Model: RQI versus Hold Time; Actual Temp Method}

Factor coding $(-1 ; 0 ;+1)$

\section{Factor Information}

\begin{tabular}{llrl} 
Factor & Type & Levels & Values \\
\hline Hold Time & Fixed & 3 & $15 ; 20 ; 25$ \\
Actual Temp & Fixed & 5 & $573 ; 582 ; 585 ; 592 ; 595$
\end{tabular}

Analysis of Variance

\begin{tabular}{lrrrrr} 
Source & DF & Adj SS & Adj MS & F-Value & P-Value \\
\hline Hold Time & 2 & 0.07954 & 0.039770 & 9.70 & 0.000 \\
Actual Temp & 4 & 0.04315 & 0.010789 & 2.63 & 0.046 \\
Hold Time*Actual Temp & 8 & 0.03178 & 0.003972 & 0.97 & 0.471
\end{tabular}




$\begin{array}{llll}\text { Error } & 48 & 0.19685 & 0.004101 \\ \text { Total } & 62 & 0.36334 & \end{array}$

Model Summary

\begin{tabular}{rrrr}
$S$ & R-sq & R-sq(adj) & R-sq(pred) \\
\hline 0.0640387 & $45.82 \%$ & $30.02 \%$ & $0.00 \%$
\end{tabular}

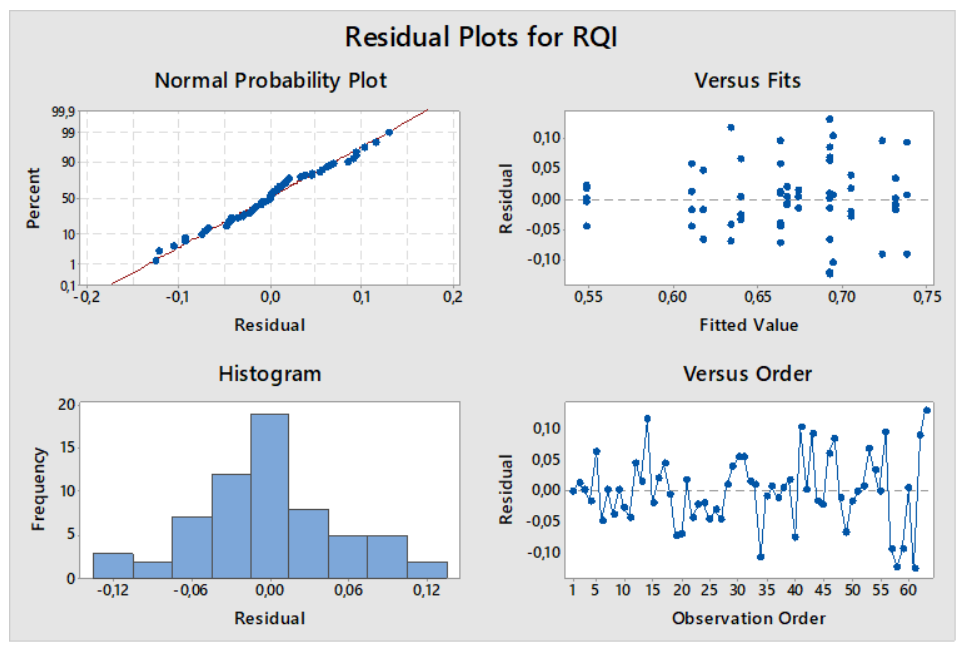

Figure 105 - Residual Plot for the A356 hold time and temperature effects on RQI

Besides the RQI, the Grain Size / Diameter of the semi-solid alloy is important especially for the size of the nozzle neck, the smaller diameter channel and outlet (see Finke [71]).

Below ANOVA shows the Grain Size (Diameter calculated Diameter = $4^{*}$ Area/Perimeter) is only affected by the hold time and not the temperature or interaction.

\section{General Linear Model: Diameter $(D=4 A / P)$ versus Hold Time; Actual Temperature \\ Factor Information}

\begin{tabular}{|c|c|c|c|c|c|}
\hline Factor & Type & Levels & Values & & \\
\hline Hold Time & Fixed & 3 & $15 ; 20 ; 25$ & & \\
\hline Actual Temp & Fixed & 5 & $573 ; 582 ; 585 ; 592 ;$ & 595 & \\
\hline \multicolumn{6}{|c|}{ Analysis of Variance } \\
\hline Source & & DF & Adj MS & F-Value & P-Value \\
\hline
\end{tabular}




$\begin{array}{lrrrrr}\text { Hold Time } & 2 & 1052.6 & 526.31 & 7.39 & 0.002 \\ \text { Actual Temp } & 4 & 379.6 & 94.91 & 1.33 & 0.271 \\ \text { Hold Time*Actual Temp } & 8 & 715.3 & 89.42 & 1.26 & 0.289 \\ \text { Error } & 48 & 3417.5 & 71.20 & & \\ \text { Total } & 62 & 5472.2 & & & \end{array}$

\section{Model Summary}

\begin{tabular}{rrrr} 
S & R-sq & R-sq(adj) & R-sq(pred) \\
\hline 8.43787 & $37.55 \%$ & $19.33 \%$ & $0.00 \%$
\end{tabular}

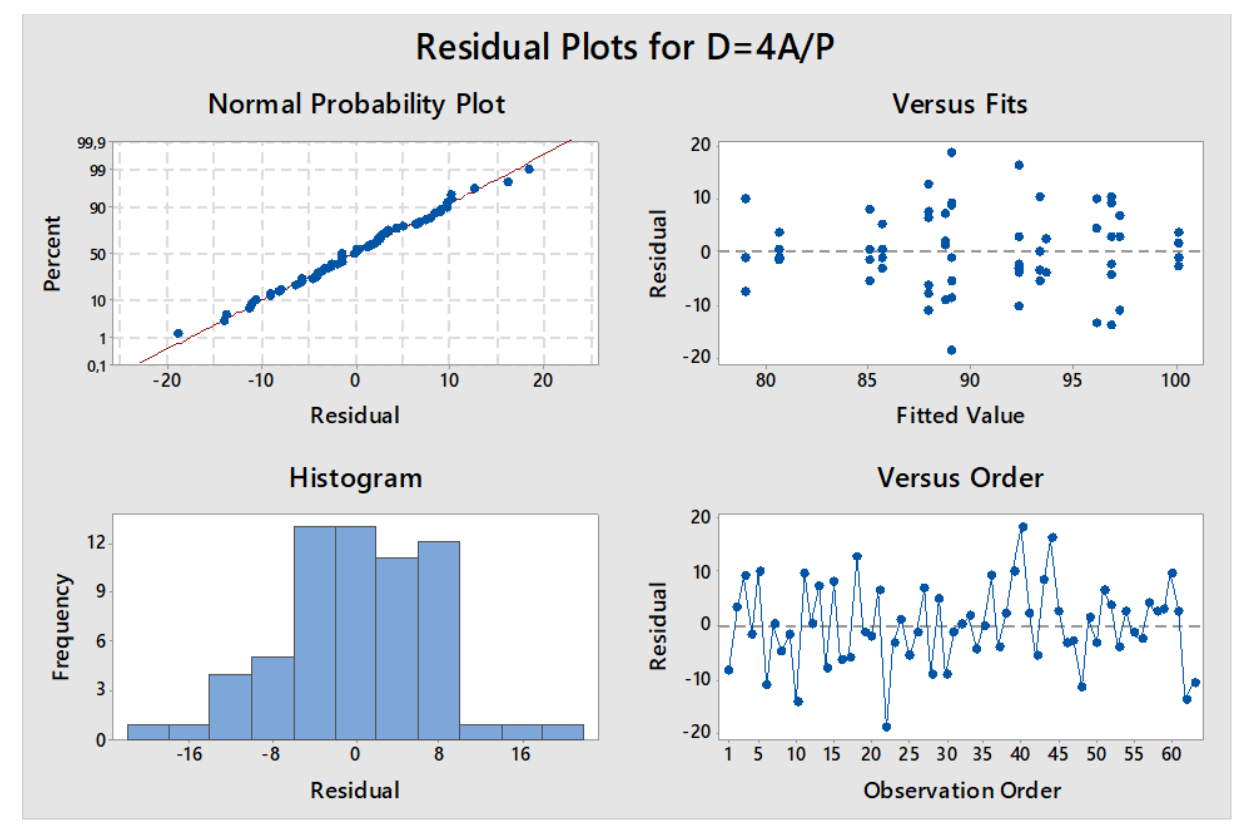

Figure 106 - A356 Residual Plots for effects on Grain Size

The fraction solid is not determined by any of the factors as shown below:

\section{General Linear Model: FS versus Hold Time; Actual Temp Factor Information}

\begin{tabular}{llrl} 
Factor & Type & Levels & Values \\
\hline Hold Time & Fixed & 3 & $15 ; 20 ; 25$ \\
Actual Temp & Fixed & 5 & $573 ; 582 ; 585 ; 592 ; 595$
\end{tabular}

Analysis of Variance

\begin{tabular}{lrrrrr} 
Source & DF & Adj SS & Adj MS & F-Value & P-Value \\
\hline Hold Time & 2 & 20.88 & 10.44 & 0.36 & 0.701
\end{tabular}




$\begin{array}{lrrrrr}\text { Actual Temp } & 4 & 234.23 & 58.56 & 2.01 & 0.109 \\ \text { Hold Time*Actual Temp } & 8 & 354.44 & 44.31 & 1.52 & 0.176 \\ \text { Error } & 48 & 1401.75 & 29.20 & & \\ \text { Total } & 62 & 2033.20 & & & \end{array}$

\section{Model Summary}

\begin{tabular}{rrrr} 
S & R-sq & R-sq(adj) & R-sq(pred) \\
\hline 5.40399 & $31.06 \%$ & $10.95 \%$ & $0.00 \%$
\end{tabular}

The THIXALLOY 540 has been reviewed with a single setting temperature and as such with only 3 observed temperatures as per model in Table 20. Overall as shown in the below ANOVA tables the RQI is only affected by the hold time. The Grain Size / Diameter is affected in contrast to A356 by the hold time and temperature.

\section{General Linear Model: RQI versus Hold Time; Actual Temp}

\section{Factor Information}

\begin{tabular}{llll} 
Factor & Type & Levels & Values \\
\hline Hold Time & Fixed & 3 & $15 ; 20 ; 25$ \\
Actual Temp & Fixed & 3 & $593 ; 602 ; 605$ \\
Analysis of & Variance
\end{tabular}

\begin{tabular}{lrrrrr} 
Source & DF & Adj SS & Adj MS & F-Value & P-Value \\
\hline Hold Time & 2 & 0.043083 & 0.021541 & 4.22 & 0.027 \\
Actual Temp & 2 & 0.002275 & 0.001137 & 0.22 & 0.802 \\
Hold Time*Actual Temp & 4 & 0.005026 & 0.001256 & 0.25 & 0.909 \\
Error & 24 & 0.122484 & 0.005104 & & \\
Total & 32 & 0.170887 & & &
\end{tabular}

Model Summary

\begin{tabular}{rrrr} 
S & R-sq & R-sq(adj) & R-sq(pred) \\
\hline 0.0714388 & $28.32 \%$ & $4.43 \%$ & $0.00 \%$
\end{tabular}




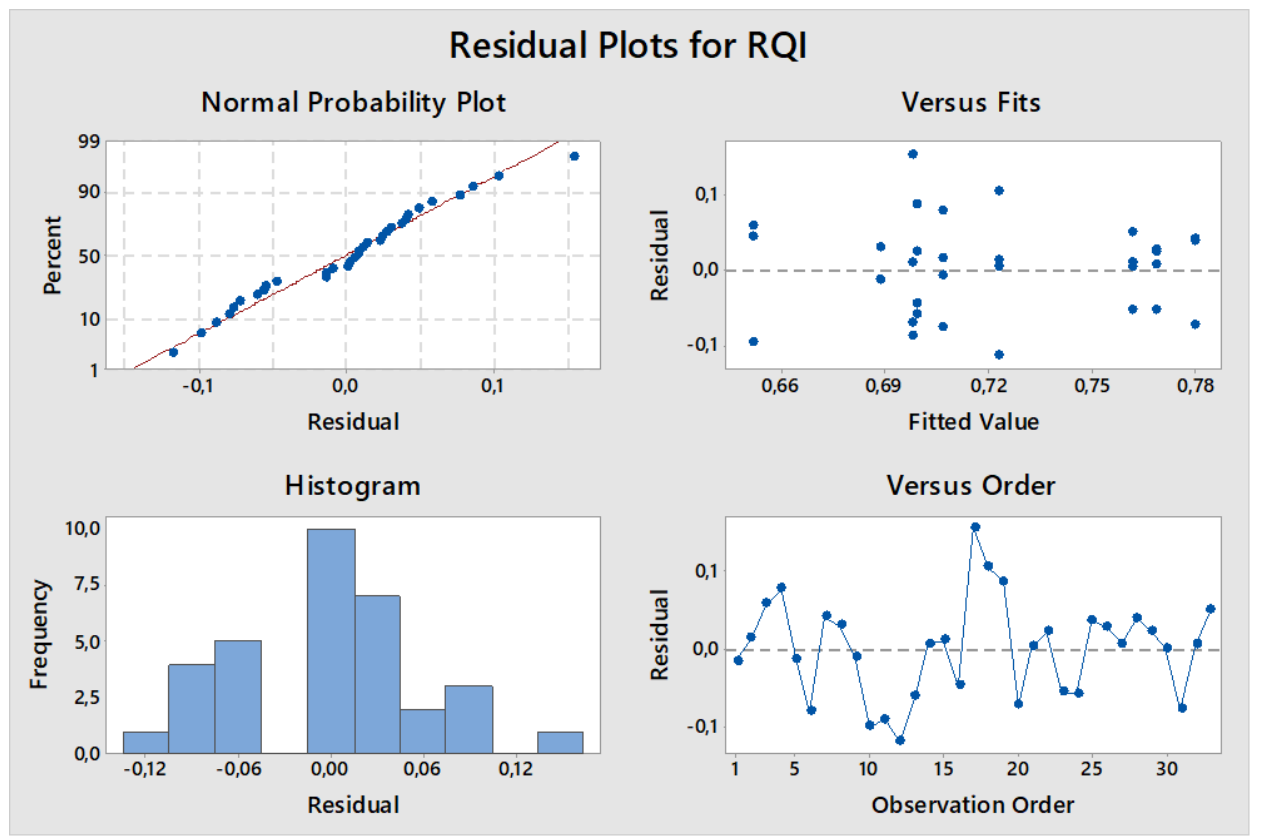

Figure 107 - THIXALLOY 540 Residuals Plot for RQI

\section{General Linear Model: Diameter $(D=4 A / P)$ versus Actual Temperature; Hold Time}

\section{Factor Information}

\begin{tabular}{llrl} 
Factor & Type & Levels & Values \\
\hline Actual Temp & Fixed & 3 & 593; 602;605 \\
Hold Time & Fixed & 3 & $15 ; 20 ; 25$
\end{tabular}

Analysis of Variance

\begin{tabular}{lrrrrr} 
Source & DF & Adj SS & Adj MS & F-Value & P-Value \\
\hline Actual Temp & 2 & 223.30 & 111.649 & 5.81 & 0.008 \\
Hold Time & 2 & 317.89 & 158.946 & 8.26 & 0.002 \\
Error & 28 & 538.50 & 19.232 & & \\
Lack-of-Fit & 4 & 9.86 & 2.466 & 0.11 & 0.977 \\
Pure Error & 24 & 528.63 & 22.026 & & \\
Total & 32 & 1095.92 & & &
\end{tabular}

\section{Model Summary}

\begin{tabular}{rrrr} 
S & R-sq & R-sq(adj) & R-sq(pred) \\
\hline 4.38544 & $50.86 \%$ & $43.84 \%$ & $31.98 \%$
\end{tabular}



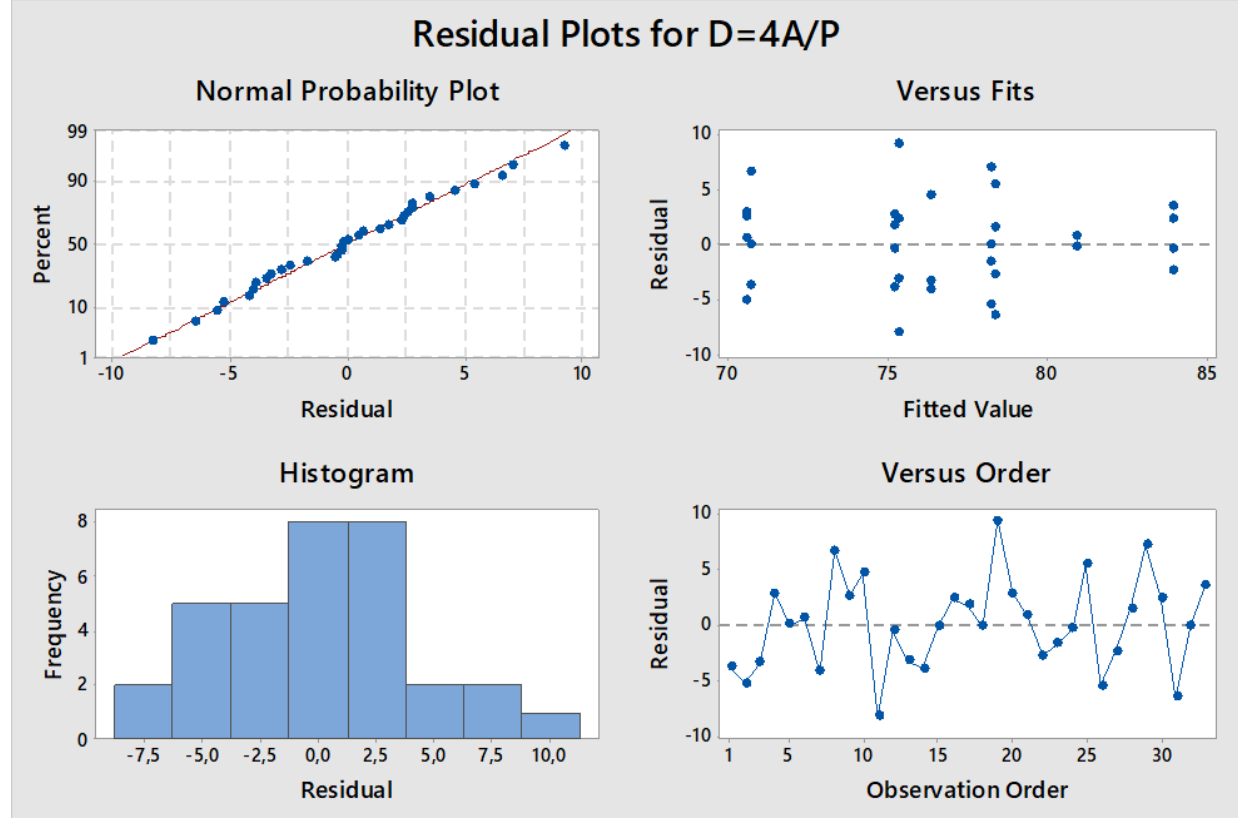

Figure 108 - THIXALLOY 540 Residuals Plot for Grain Size / Diameter

\subsubsection{Aging experiment}

The aging experiment was designed to understand the time-based development of the microstructure within the filament after a semi-solid processing state has been reached. In practical terms for MEAM it would be significant for a re-heating of an already globular material for immediate extrusion. In case of a resistive heating approach the filament may observe waiting time at the semi solid state due to non-extruding position changes of the nozzle, waiting time to start printing, etc. It needs to be understood at what holding time the microstructure is deemed outside the processing parameter. It is expected (see section 2.1.4 and Figure 7) that the main effect is coarsening but not creation of a dendritic structure. In addition, it needs to be observed whether the RQI will drop over time. It is expected that if the roundness of coarsened grains (see Figure $7 f$ ) is even more globular the $\mathrm{RQI}$ will not drop but increase.

The experiment process and analyses are identical to the heating experiment. Meaningful timeframes to test are $30 \mathrm{~min}(5 \mathrm{~min}$ after the longest heating experiment), $40 \mathrm{~min}$ and $50 \mathrm{~min}$. Based on the expectation that heating to globular structure is not greater than $25 \mathrm{~min}$, a holding time review between $5 \mathrm{~min}$ and $25 \mathrm{~min}$ is assumed meaningful for MEAM machines operation. 
As the holding time intervals are greater than the ones used in the heating experiment the maximum difference for the sample / power calculation can be relaxed. From the below Minitab Power and Sample Size calculation it has been assumed that a maximum difference of $4 \mu \mathrm{m}$ in grain size is sufficient. As such that sample size has been chosen as 2 replicates (see also Figure 109).

\section{Power and Sample Size}

General Full Factorial Design

$\alpha=0.05$ Assumed standard deviation $=0.966$

Factors: 2 Number of levels: 3; 2

Include terms in the model up through order: 2

Not including blocks in model.

\section{Results}

\begin{tabular}{rrrrr}
$\begin{array}{r}\text { Maximum } \\
\text { Difference }\end{array}$ & Reps & $\begin{array}{r}\text { Total } \\
\text { Runs }\end{array}$ & $\begin{array}{r}\text { Target } \\
\text { Power }\end{array}$ & Actual Power \\
\hline 3 & 3 & 18 & 0.90 & 0.991592 \\
3 & 3 & 18 & 0.95 & 0.991592 \\
4 & 2 & 12 & 0.90 & 0.981303 \\
4 & 2 & 12 & 0.95 & 0.981303
\end{tabular}

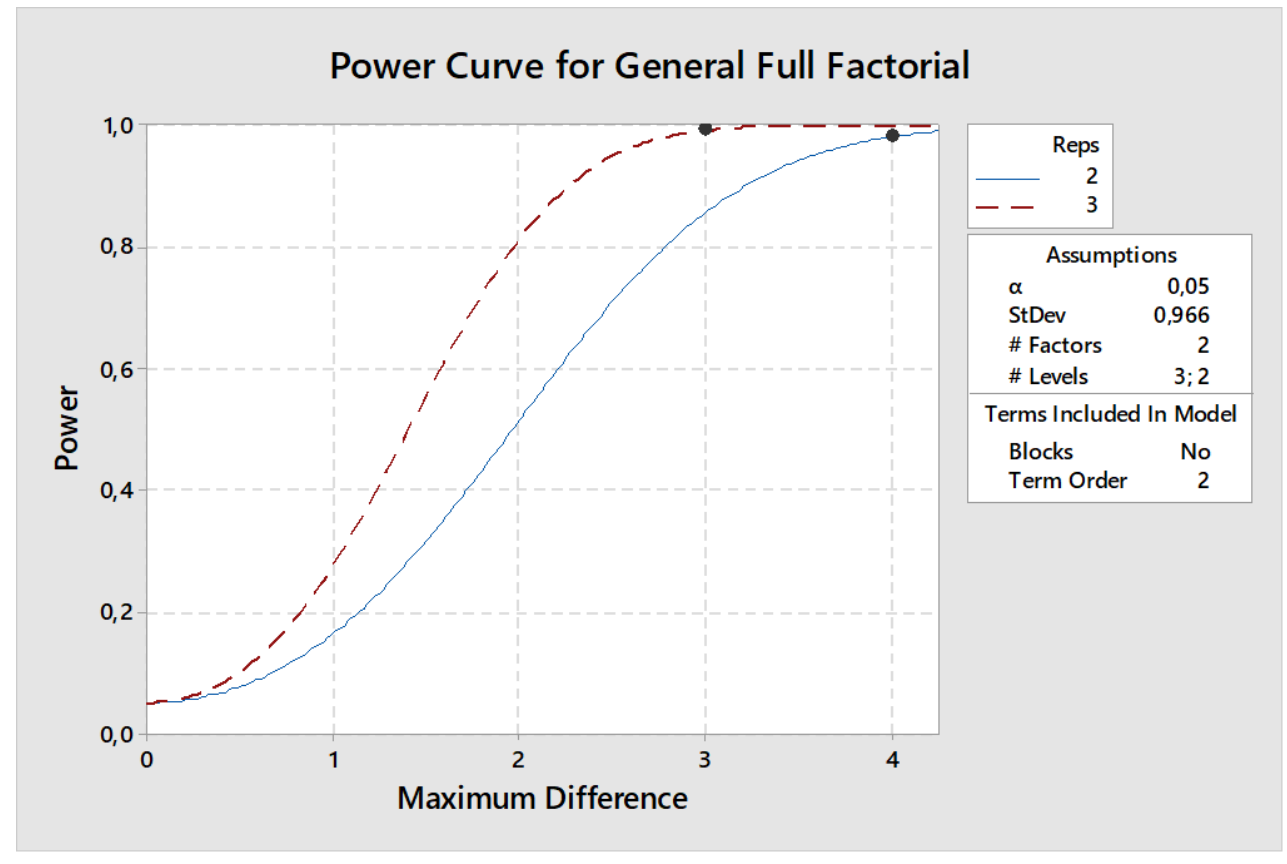

Figure 109 - Power Curve for full factorial design for the aging experiment

The full factorial randomized design is shown in Appendix 0 . 
For the same reason as in the heating experiment the lower temperature for the reference alloy (THIXALLOY 540) has been removed from the experiment.

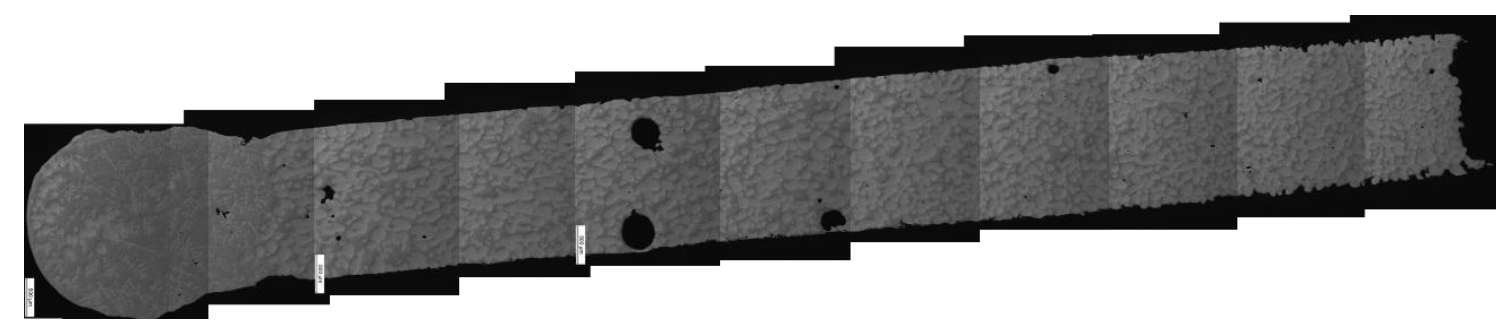

Figure 110 - Aging example - sample 19: A356 50min @ 585 C

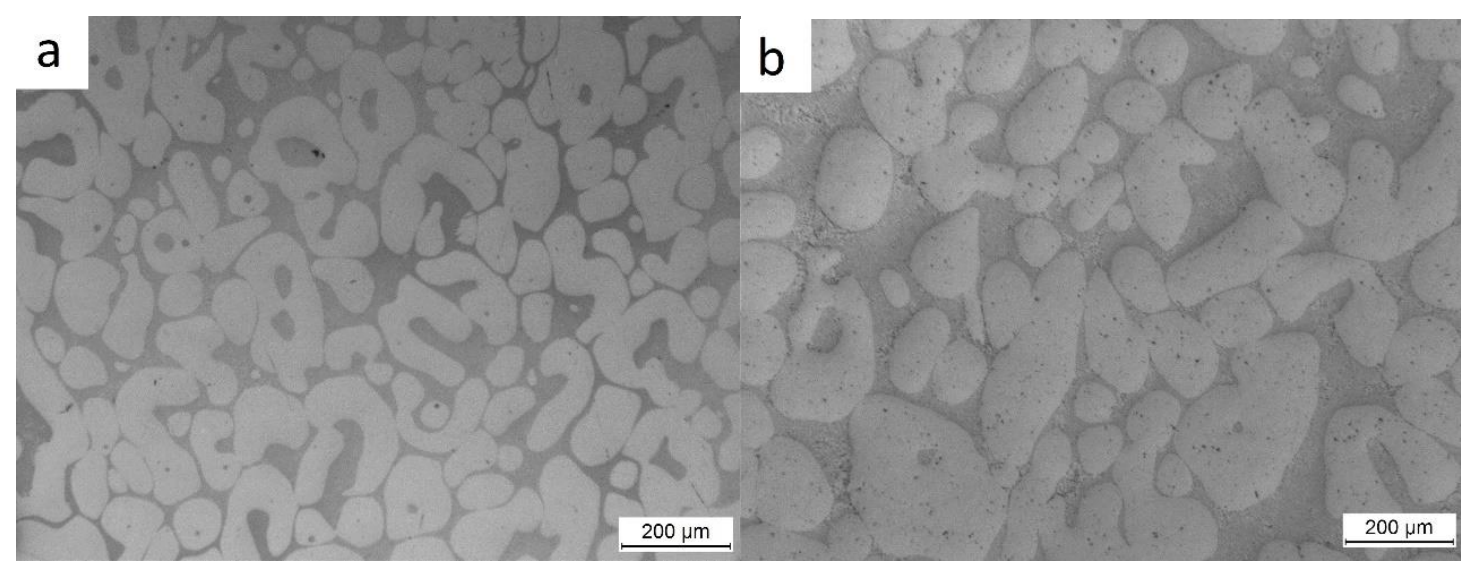

Figure 111 - A356 a) Sample 18M - 15min @ 585 $\mathrm{C}$ b) Sample 19M 50min @ 585 $\mathrm{C}$

As shown below (see ANOVA and Figure 113) the RQI does increase but stabilize over time for A356 and increase over time for THIXALLOY 540.

\section{General Linear Model: A356 aging RQI versus Hold Time; Actual Temperature}

\section{Factor Information}

\begin{tabular}{llrl} 
Factor & Type & Levels & Values \\
\hline Hold Time & Fixed & 6 & $15 ; 20 ; 25 ; 30 ; 40 ; 50$ \\
Actual Temp & Fixed & 5 & $573 ; 582 ; 585 ; 592 ; 595$
\end{tabular}




\section{Analysis of Variance}

\begin{tabular}{lrrrrr} 
Source & DF & Adj SS & Adj MS & F-Value & P-Value \\
\hline Hold Time & 5 & 0.16338 & 0.032677 & 10.03 & 0.000 \\
Actual Temp & 4 & 0.04463 & 0.011157 & 3.42 & 0.012 \\
Error & 83 & 0.27043 & 0.003258 & & \\
Lack-of-Fit & 19 & 0.06895 & 0.003629 & 1.15 & 0.325 \\
Pure Error & 64 & 0.20148 & 0.003148 & & \\
Total & 92 & 0.47038 & & &
\end{tabular}

\section{Model Summary}

\begin{tabular}{rrrr} 
S & R-sq & R-sq(adj) & R-sq(pred) \\
\hline 0.0570804 & $42.51 \%$ & $36.27 \%$ & $28.38 \%$
\end{tabular}

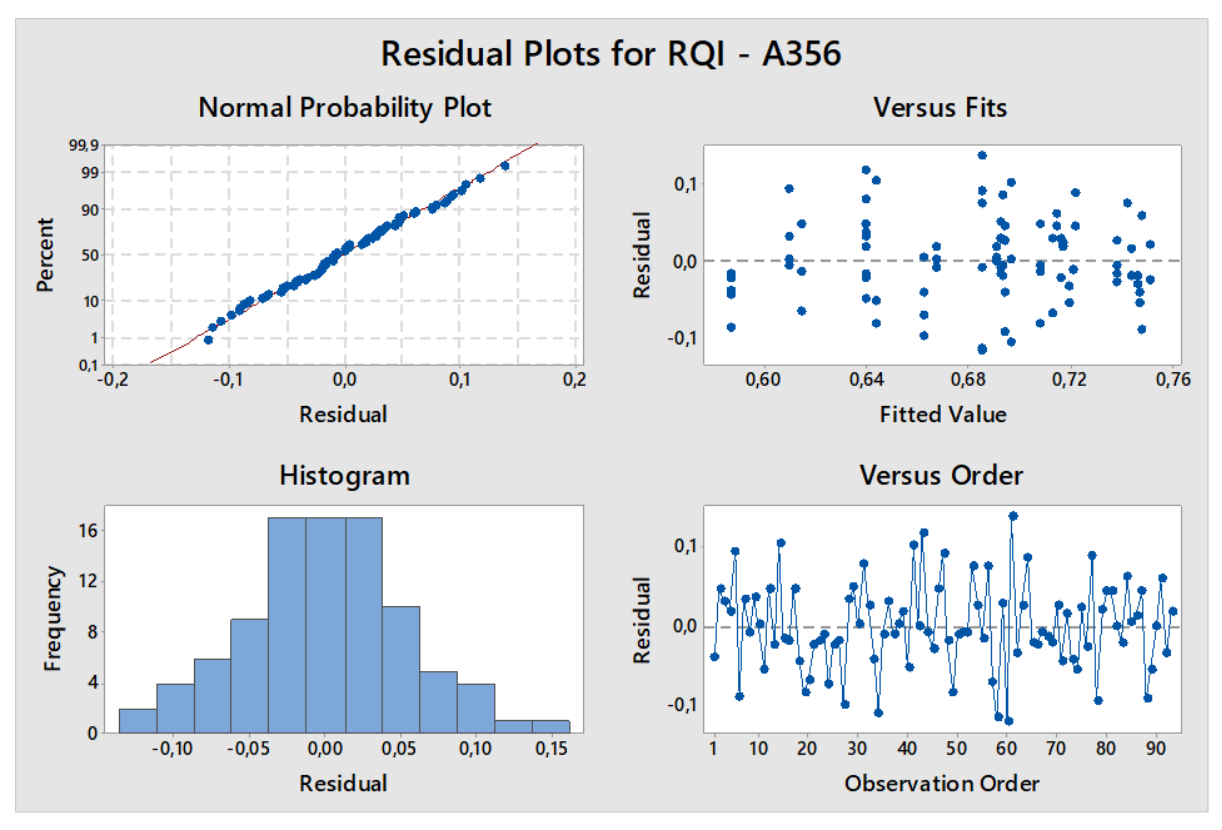

Figure 112 - Residual Plot for A356 heating to aging 


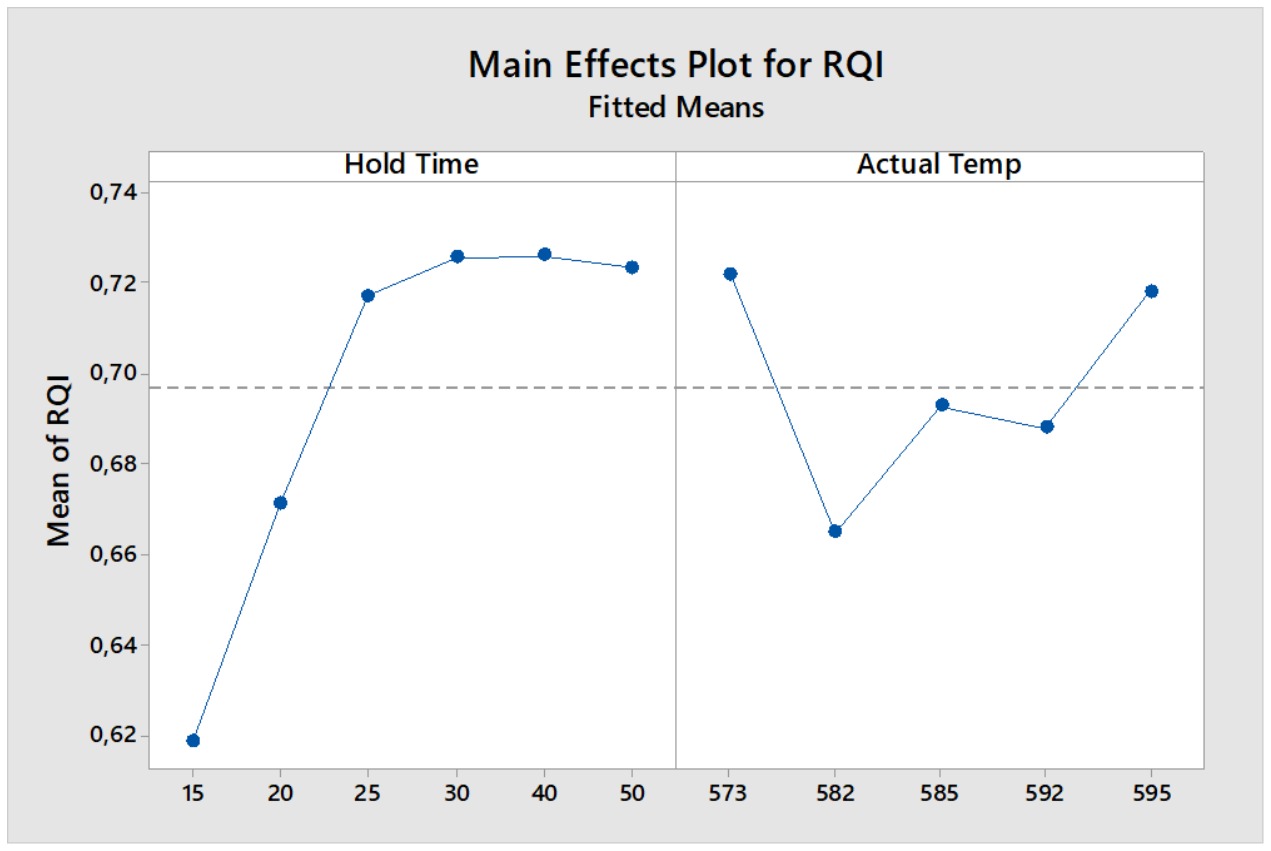

Figure 113 - Main effects plot for RQI - A356 heating to aging

\section{General Linear Model: RQI versus Hold Time; Actual Temp}

\section{Factor Information}

\begin{tabular}{llrl} 
Factor & Type & Levels & Values \\
\hline Hold Time & Fixed & 6 & $15 ; 20 ; 25 ; 30 ; 40 ; 50$ \\
Actual Temp & Fixed & 3 & $593 ; 602 ; 605$
\end{tabular}

\section{Analysis of Variance}

\begin{tabular}{lrrrrr} 
Source & DF & Adj SS & Adj MS & F-Value & P-Value \\
\hline Hold Time & 5 & 0.164863 & 0.032973 & 7.35 & 0.000 \\
Actual Temp & 2 & 0.005539 & 0.002770 & 0.62 & 0.546 \\
Hold Time*Actual Temp & 10 & 0.017675 & 0.001767 & 0.39 & 0.939 \\
Error & 30 & 0.134639 & 0.004488 & & \\
Total & 47 & 0.320243 & & &
\end{tabular}

\section{Model Summary}

\begin{tabular}{rrrr} 
S & R-sq & R-sq(adj) & R-sq(pred) \\
\hline 0.0669923 & $57.96 \%$ & $34.13 \%$ & $*$
\end{tabular}




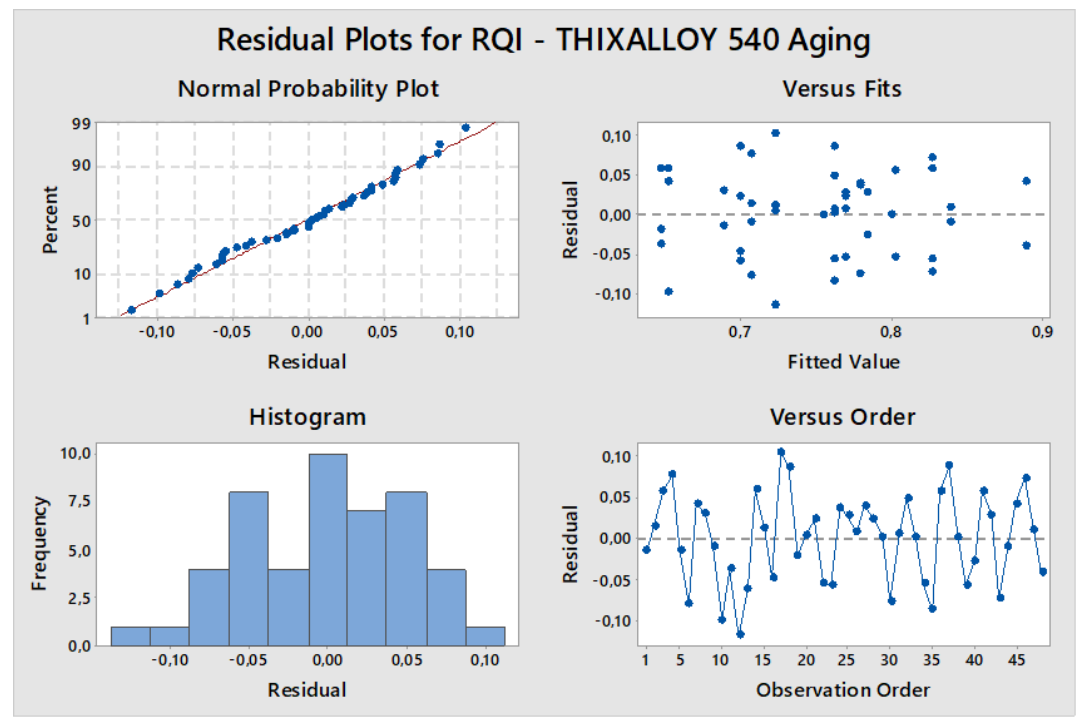

Figure 114 - Residuals Plot RQI - THIXALLOY 540 Aging

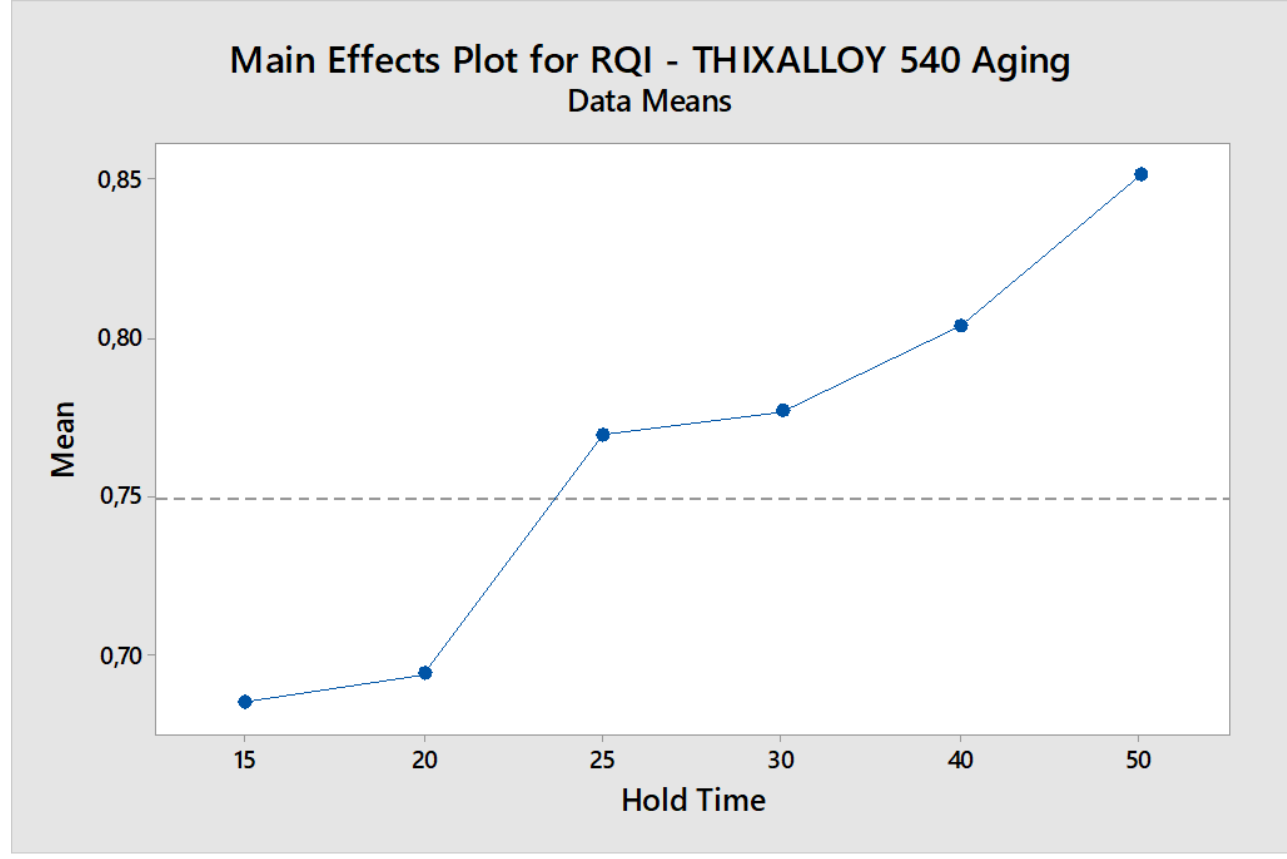

Figure 115 - Main effects plot for RQI - THIXALLOY 540 Aging

As per ANOVA below the grain size/diameter is only affected by the holding time. As such a regression analysis has been done to study the behavior throughout the time sampled.

\section{General Linear Model: A356 Diameter versus Hold Time; Actual Temperature}




\section{Factor Information}

\begin{tabular}{llrl} 
Factor & Type & Levels & Values \\
\hline Hold Time & Fixed & 6 & $15 ; 20 ; 25 ; 30 ; 40 ; 50$ \\
Actual Temp & Fixed & 5 & $573 ; 582 ; 585 ; 592 ; 595$ \\
Analysis of & Variance
\end{tabular}

\begin{tabular}{lrrrrr} 
Source & DF & Adj SS & Adj MS & F-Value & P-Value \\
\hline Hold Time & 5 & 6902.5 & 1380.50 & 18.89 & 0.000 \\
Actual Temp & 4 & 228.9 & 57.22 & 0.78 & 0.539 \\
Error & 83 & 6066.1 & 73.09 & & \\
Lack-of-Fit & 19 & 1328.4 & 69.91 & 0.94 & 0.534 \\
Pure Error & 64 & 4737.7 & 74.03 & & \\
Total & 92 & 13156.2 & & & \\
Model Summary & & & &
\end{tabular}

\begin{tabular}{rrrr} 
S & R-sq & R-sq(adj) & R-sq(pred) \\
\hline 8.54898 & $53.89 \%$ & $48.89 \%$ & $42.62 \%$
\end{tabular}

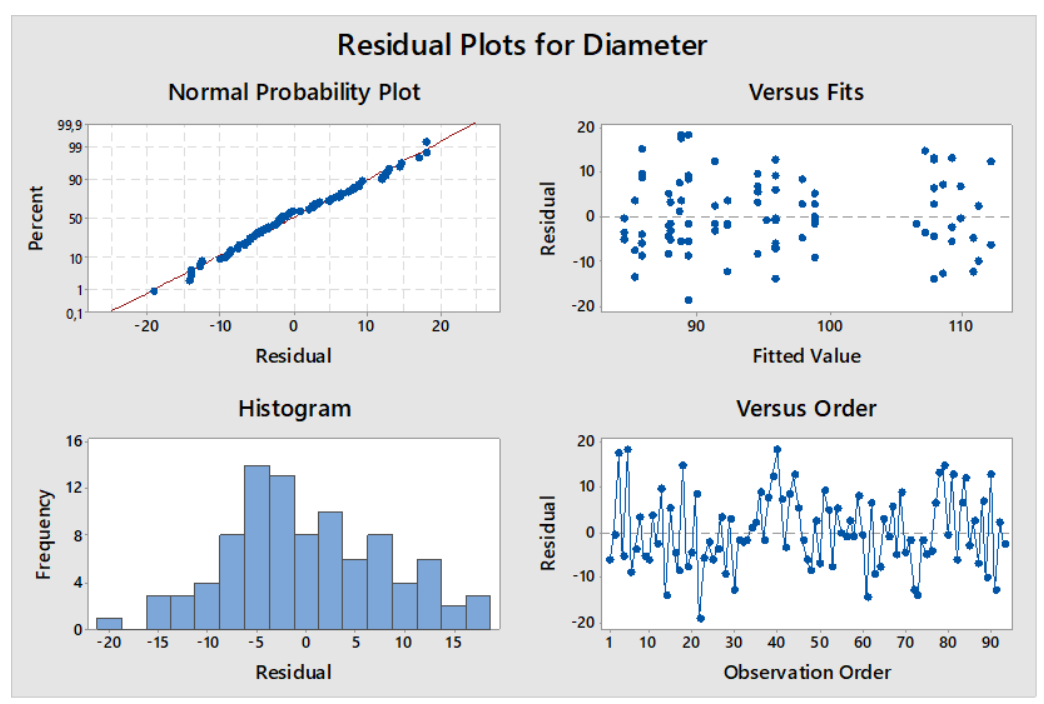

Figure 116 - Residual Plot 


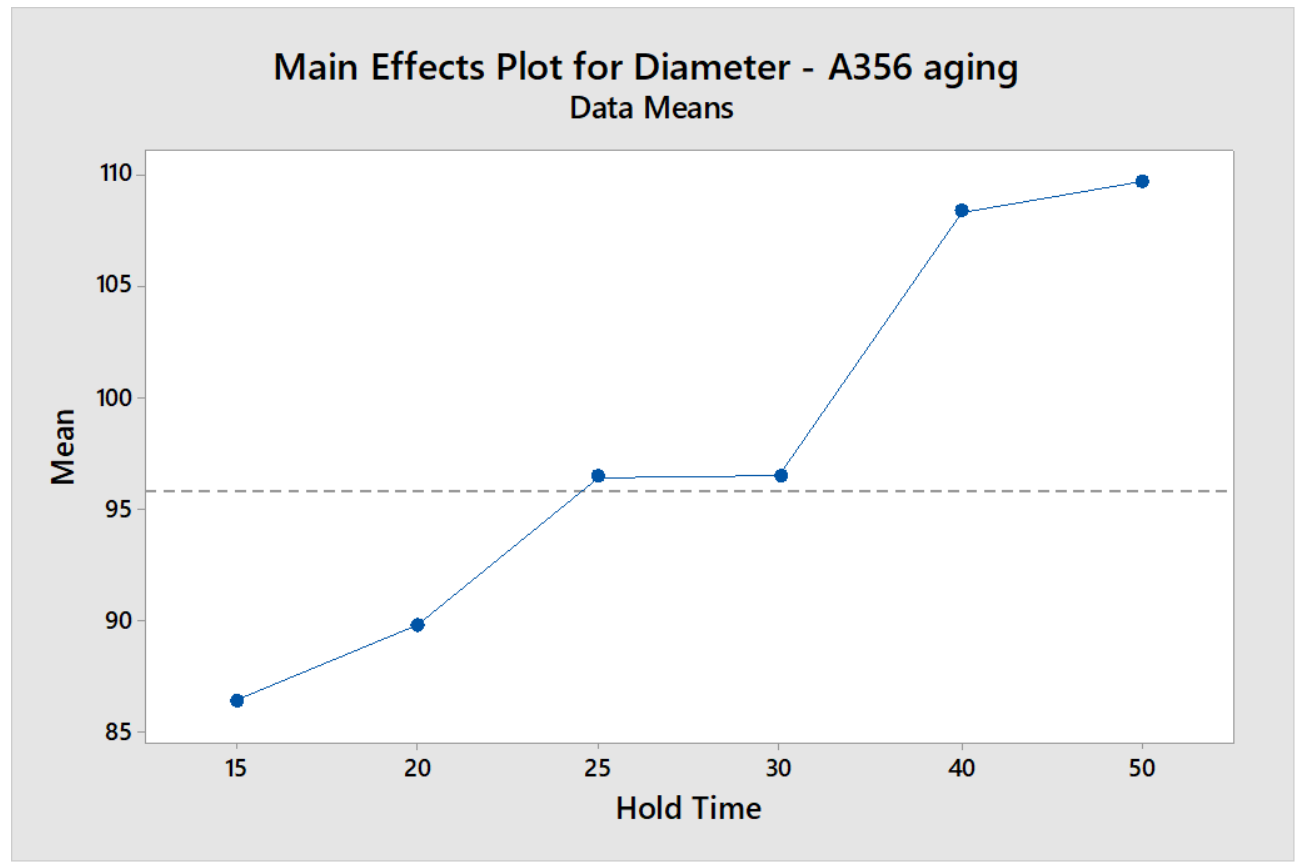

Figure 117 - Main effects plot for grain size - A356 aging

\section{Regression Analysis: A356 Diameter versus Hold Time} Analysis of Variance

\begin{tabular}{lrrrrr} 
Source & DF & Adj SS & Adj MS & F-Value & P-Value \\
\hline Regression & 1 & 6525.5 & 6525.54 & 89.56 & 0.000 \\
Hold Time & 1 & 6525.5 & 6525.54 & 89.56 & 0.000 \\
Error & 91 & 6630.7 & 72.86 & & \\
Lack-of-Fit & 4 & 335.7 & 83.93 & 1.16 & 0.334 \\
Pure Error & 87 & 6294.9 & 72.36 & & \\
Total & 92 & 13156.2 & & &
\end{tabular}

\section{Model Summary}

\begin{tabular}{rrrr} 
S & R-sq & R-sq(adj) & R-sq(pred) \\
\hline 8.53607 & $49.60 \%$ & $49.05 \%$ & $47.21 \%$
\end{tabular}

\section{Coefficients}

\begin{tabular}{lrrrrr} 
Term & Coef & SE Coef & T-Value & P-Value & VIF \\
\hline Constant & 76.31 & 2.24 & 33.99 & 0.000 & \\
Hold Time & 0.7191 & 0.0760 & 9.46 & 0.000 & 1.00
\end{tabular}

\section{Regression Equation}

$$
\text { Diameter }=76.31+0.7191 \text { Hold Time }
$$




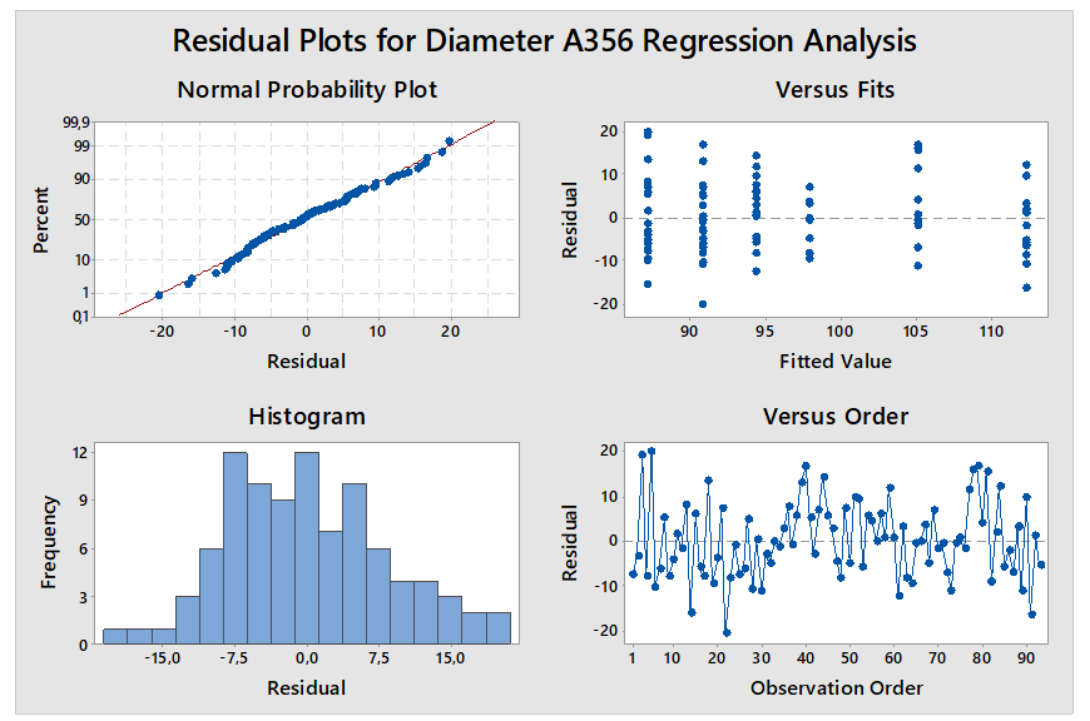

Figure 118 - Residual Plot for Regression Analysis Grain Size - A356 Aging

\section{General Linear Model: THIXALLOY 540 - Diameter versus Hold Time; Actual Temp}

\section{Factor Information}

\begin{tabular}{lrrrrrr} 
Factor & Type & Levels & \multicolumn{2}{l}{ Values } & & \\
\cline { 1 - 5 } Hold Time & Fixed & 6 & $15 ; 20 ; 25 ; 30 ; 40 ; 50$ & & \\
Actual Temp & Fixed & 3 & $593 ; 602 ; 605$ & & \\
Analysis of Variance & & & & & \\
Source & DF & Adj SS & Adj MS & F-Value & P-Value \\
\hline Hold Time & 5 & 2952.74 & 590.548 & 26.21 & 0.000 \\
Actual Temp & 2 & 449.67 & 224.834 & 9.98 & 0.001 \\
Hold Time*Actual Temp & 10 & 70.68 & 7.068 & 0.31 & 0.971 \\
Error & 29 & 653.42 & 22.532 & & \\
Total & 46 & 4295.29 & & &
\end{tabular}

Model Summary

\begin{tabular}{rrrr} 
S & R-sq & R-sq(adj) & R-sq(pred) \\
\hline 4.74678 & $84.79 \%$ & $75.87 \%$ & $*$
\end{tabular}




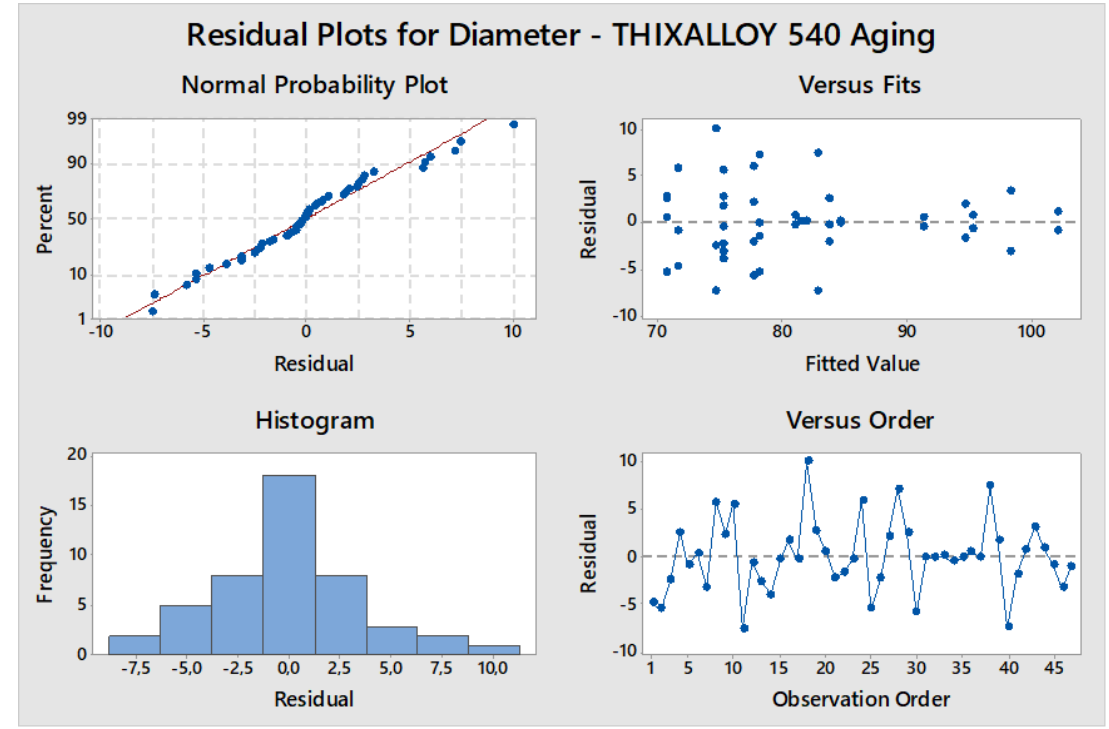

Figure 119 - Residuals Plot for Diameter - THIXALLOY 540 Aging

While for THIXALLOY the grain size is affected by both holding time and temperature, it is assumed that temperature can be removed when the factor levels would be increased similar to A356. Also to note is that Residual vs Fitted Value show very small spread of residuals for larger average grain size. For verification to A356 the regression analysis has been done for holding time only. With the below regression model $(\mathrm{R}$-sq $=71 \%$ ) as well as the scatter plot for diameter (see Figure 121 - against Holding Time and Figure 122 against temperature) it is assumed that the regression against the holding time is adequate and comparable to $\mathrm{A} 356$.

\section{Regression Analysis: Diameter versus Hold Time} Analysis of Variance

\begin{tabular}{lrrrrr} 
Source & DF & Adj SS & Adj MS & F-Value & P-Value \\
\hline Regression & 1 & 3036.0 & 3036.01 & 108.49 & 0.000 \\
Hold Time & 1 & 3036.0 & 3036.01 & 108.49 & 0.000 \\
Error & 45 & 1259.3 & 27.98 & & \\
Lack-of-Fit & 4 & 111.2 & 27.81 & 0.99 & 0.422 \\
Pure Error & 41 & 1148.1 & 28.00 & & \\
Total & 46 & 4295.3 & & &
\end{tabular}

Model Summary 


\begin{tabular}{rrrr} 
S & R-sq & R-sq(adj) & R-sq(pred) \\
\hline 5.29000 & $70.68 \%$ & $70.03 \%$ & $68.17 \%$
\end{tabular}

\section{Coefficients}

\begin{tabular}{lrrrrr} 
Term & Coef & SE Coef & T-Value & P-Value & VIF \\
\hline Constant & 62.15 & 2.00 & 31.05 & 0.000 & \\
Hold Time & 0.7091 & 0.0681 & 10.42 & 0.000 & 1.00
\end{tabular}

\section{Regression Equation}

$$
\text { Diameter }=62.15+0.7091 \text { Hold Time }
$$

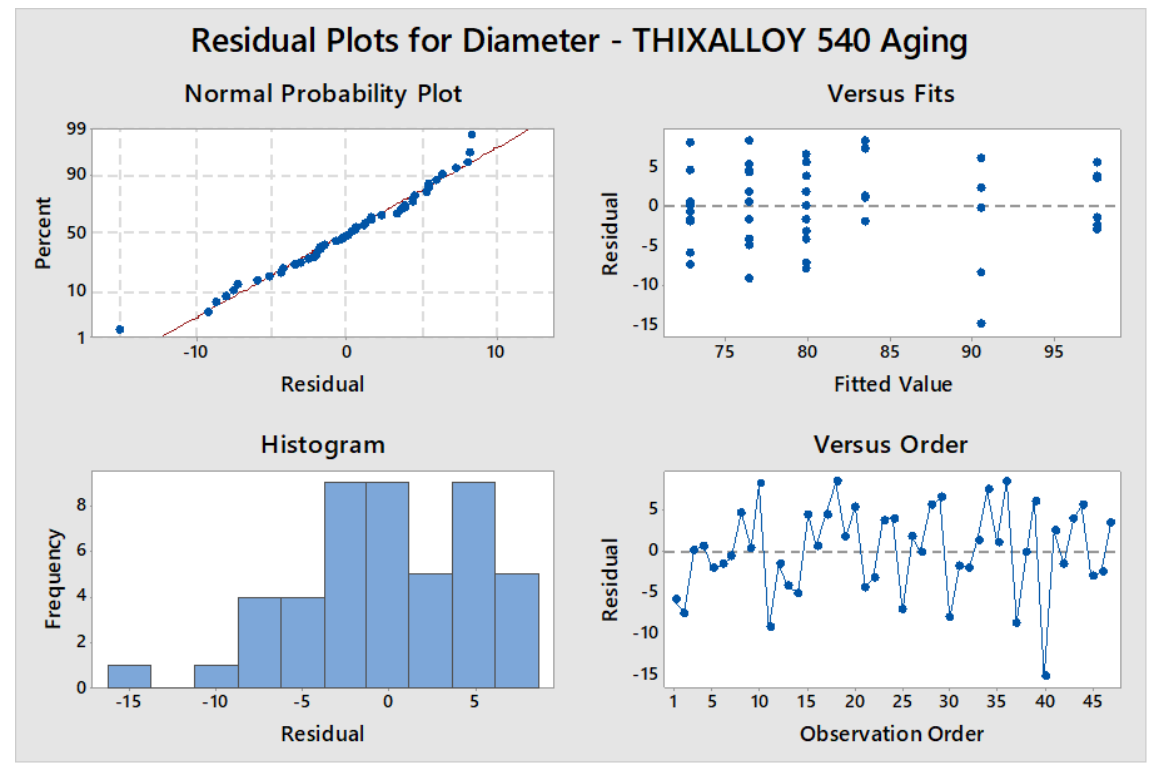

Figure 120 - Residual Plot for Regression Diameter - THIXALLOY 540 aging 


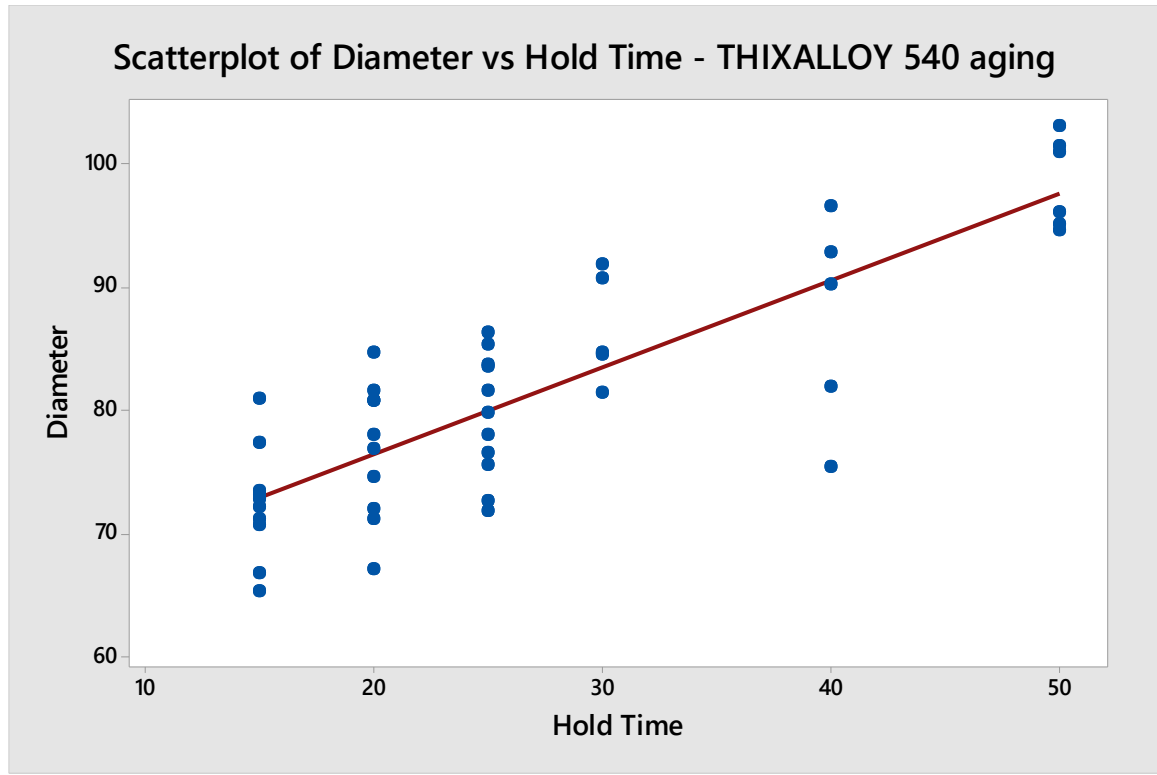

Figure 121 - Scatterplot with regression Diameter vs Hold Time - THIXALLOY 540

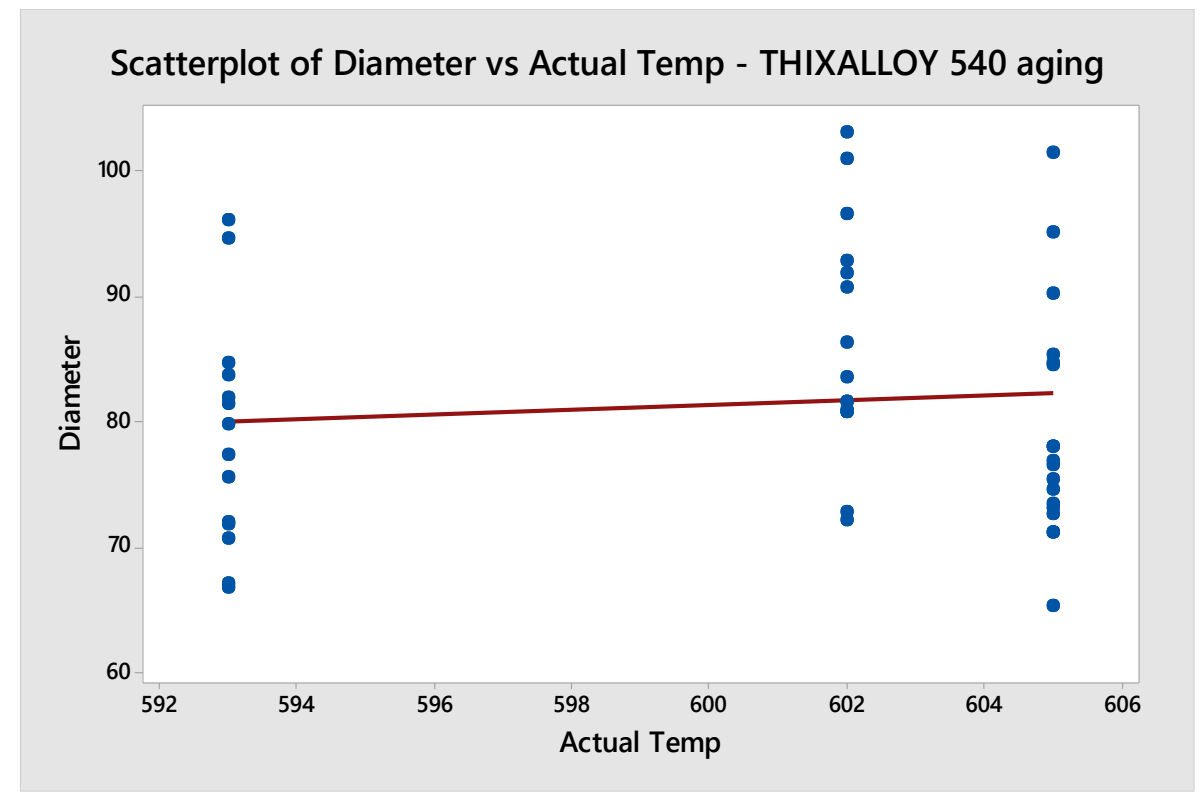

Figure 122 - Scatterplot with regression Diameter vs temperature - THIXALLOY 540

\subsubsection{Cleaning process development}

It is important to remove material remains from the nozzle especially also $\mathrm{Al}_{2} \mathrm{O}_{3}$ material as that has a far higher melting temperature $\left(2050^{\circ} \mathrm{C}\right)$ than the MEAM apparatus would generate (up to $650^{\circ} \mathrm{C}$ ). The process developed has been derived from a step performed to clean extrusion dies in extrusion molding. Activities 
described by the Institute for Extrusion molding at the Technical University of Berlin are as follows:

- Stripping of larger remains of aluminum alloy from the die

- Dissolve alloy remains in sodium hydroxide solution at elevated temperature

- Purge produced hydrogen with an exhaust duct

- Soaking of die in distilled water

The process was first reproduced using an electromagnetic mixer to ensure the solution will be moving through the smaller channel areas (see Figure 123 left). This setup resulted in cleaning times of 1 to several hours. It was assumed the process is stopped if no gas bubble is released from the nozzle channel. Later this setup was replaced by an ultra-sonic cleaning apparatus (see Figure 123 right). This reduced the time to about $10-15 \mathrm{~min}$ before the gas release stopped. Also, the soaking was performed using an ultrasonic cleaner with distilled water.

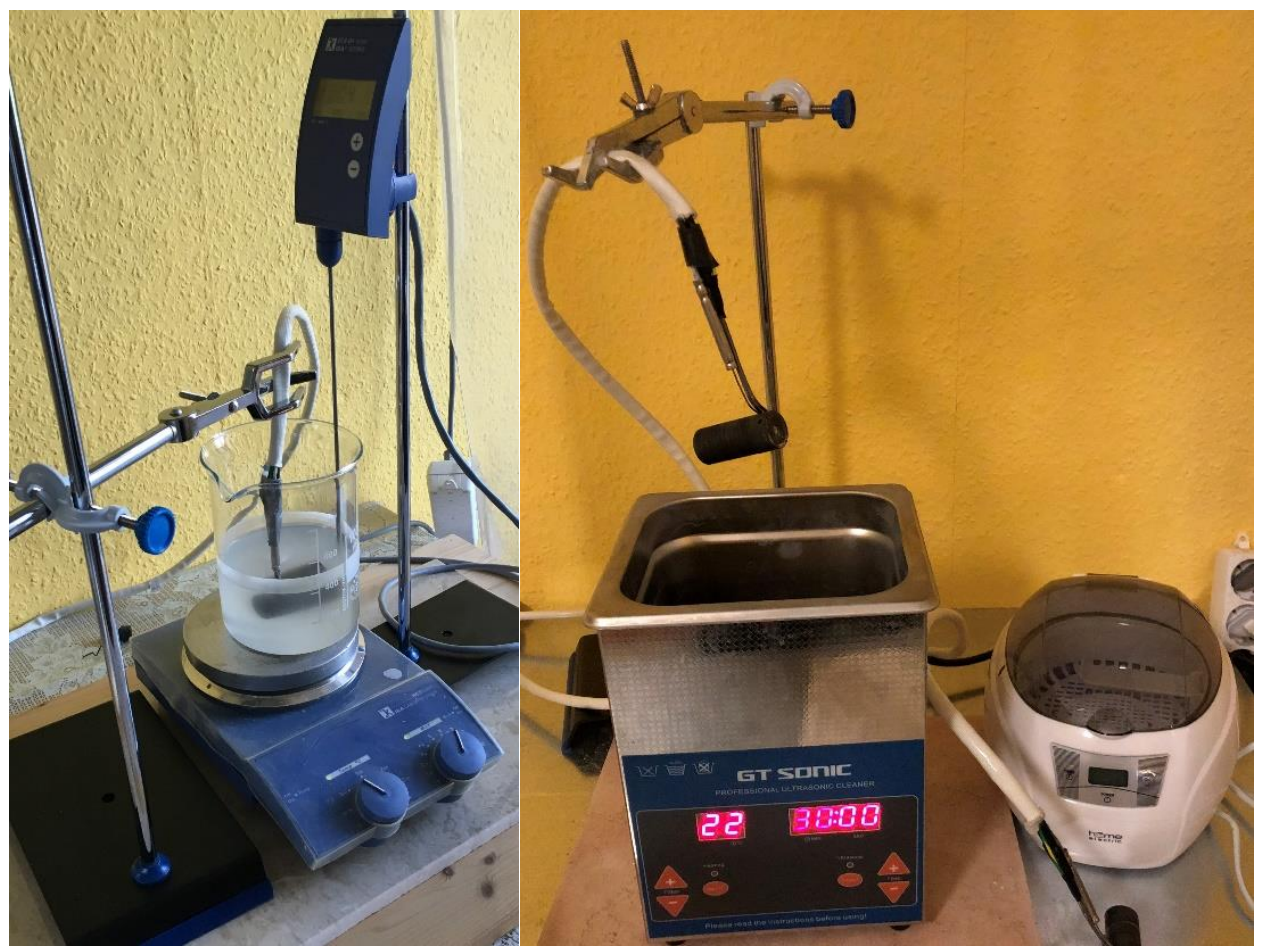

Figure 123 - Nozzle cleaning left) magnetic mixer right) ultra-sonic

All cleaning processes were performed using $50 \%$ sodium hydroxide solution. 


\subsubsection{Extrusion experiment}

To allow an extrusion experiment with the existing nozzle and its temperature distribution the following aspects need to be respected:

1. The identified heating to globular structure holding time and temperature with applicable RQI and grain size

2. Outlet orifice size so the clogging is prevented: Orifice $>>6 x$ grain size

3. Limited filament sample length to allow a relative uniform temperature throughout the nozzle motion

4. A motion and temperature regime to process a filament sample with keeping the temperature approximately constant around the filament sample

5. Ideal condition of the nozzle channel, neck and outlet orifice in terms of former alloy remains and complete boron-nitride coating

With the results of the heating and aging experiment the following parameters are chosen for design aspects 1, 2, 3 above:

Table 24 - Extrusion experiment design aspects

\begin{tabular}{|c|c|c|c|c|}
\hline Alloy & $\begin{array}{c}\text { Holding } \\
\text { Time }\end{array}$ & $\begin{array}{c}\text { Holding } \\
\text { Temperature }\end{array}$ & $\begin{array}{c}\text { Sample } \\
\text { Length }\end{array}$ & Outlet Orifice \\
\hline A356 & $25 \mathrm{~min}$ & $595^{\circ} \mathrm{C}$ & $5 \mathrm{~mm}$ & $1.1 \mathrm{~mm}$ \\
\hline $\begin{array}{c}\text { THIXALLOY } \\
540\end{array}$ & $35 \mathrm{~min}$ & $605^{\circ} \mathrm{C}$ & $5 \mathrm{~mm}$ & $1.1 \mathrm{~mm}$ \\
\hline
\end{tabular}

The temperature \& motion regime has been based on the modeled temperature from chapter 4.1.1 and prior proof of concept runs as follows:

Table 25 - Extrusion experiment process

\begin{tabular}{|l|l|}
\hline Step & Process specification \\
\hline Pre 1 & $\begin{array}{l}\text { Heating of nozzle to target temperature }\left(595^{\circ} \mathrm{C} \text { or } 605^{\circ} \mathrm{C}\right) \text { at } 3.25 \mathrm{~mm} \\
\text { depth. Thorough through heating of nozzle }->20 \mathrm{~min}\end{array}$ \\
\hline Pre 2 & Insert Filament at $30 \mathrm{~mm}$ depth with $2 \mathrm{~mm}$ steel pin and heat for $25 \mathrm{~min}$ \\
\hline 1 & Increase temperature by $5^{\circ} \mathrm{C}$ \\
\hline
\end{tabular}




\begin{tabular}{|l|l|}
\hline Step & Process specification \\
\hline 2 & Move the filament by $5 \mathrm{~mm}$ forward with $2 \mathrm{~mm}$ steel pin \\
\hline 3 & Increase the temperature by $5^{\circ} \mathrm{C}$ \\
\hline 4 & Move the filament by $5 \mathrm{~mm}$ forward with $2 \mathrm{~mm}$ steel pin \\
\hline 5 & Increase the temperature by $10^{\circ} \mathrm{C}$ \\
\hline 6 & $\begin{array}{l}\text { Move the filament into the neck } / \text { smaller channel } 2 \mathrm{~mm} \text { piston, verify } \\
\text { with the sharpened } 2 \mathrm{~mm} \text { steel piston }\end{array}$ \\
\hline 7 & Move the filament into the test tube filled with water \\
\hline
\end{tabular}

For aspect 5 the nozzle needs to be in a cleaned condition and the boron-nitride coating needs to be applied before a sample will be extruded. The current coating is applied through a spray known from aluminum welding operation to hinder spilling adhesion. Disadvantage of the spray is that the coating is easily worn out by the solid aluminum or the steel piston used to push the filament through the nozzle. To verify the extrusion a full cleaning cycle as described in section 4.2.3 is conducted and the boron-nitride spray applied. After the defined number of experiments at least two further experiment without the cleaning and coating cycle are planned.

The experiment itself will be conducted only as a conclusion whether the material can be extruded or not. As such, the outcome is a binary / categorical one. Designed experiments with binary outcome, as used in clinical trials, require a very large sample size (see Julious [121]). Due to the cost limitation the number of experiments has been chosen as 10 samples per alloy plus 2 additional without cleaning. In case the outcome includes clogging the experiment would need to be re-designed.

The metal pins are prepared as per step requiring motion (see Figure 124). Step 6 will press the semi-solid into the neck and smaller channel - as such the pin has been sharpened to form the positive neck shape. The last pin has the outlet orifice diameter of $1.1 \mathrm{~mm}$ and was replaced for each sample. 


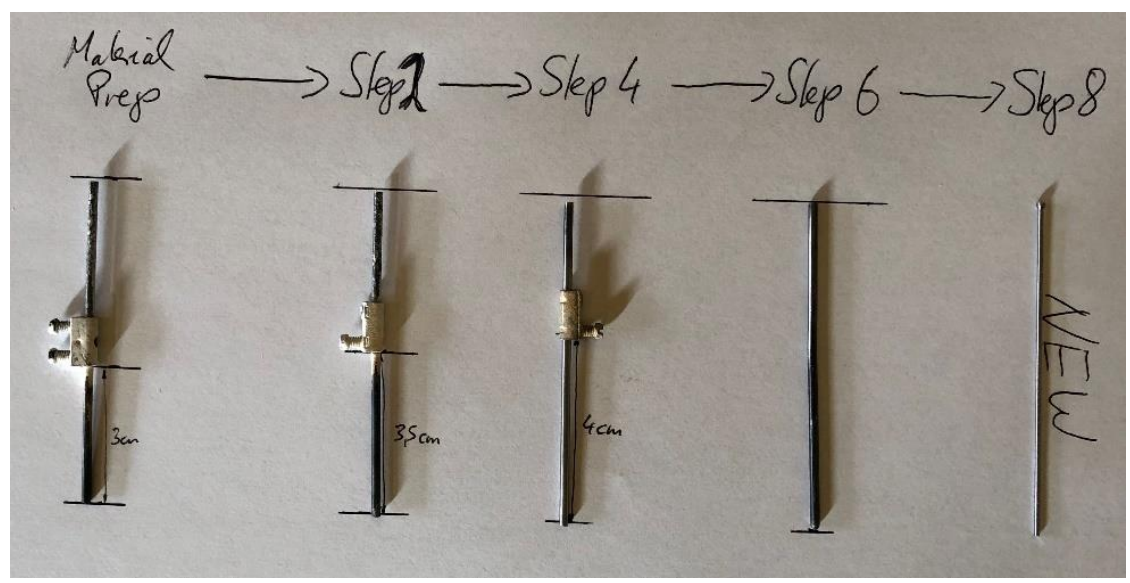

Figure 124 - Extrusion experiment piston pins

\subsubsection{Induction Pre-Heating experiment}

A quick experiment has been conducted as a proof of concept for an induction preheating of the filament to a maximum temperature of about half of the semi-solid processing temperature. For that a low cost / small-scale induction heating circuit with a 10-turn work coil has been acquired (see Figure 125). The frequency of the non-adjustable induction oscillating circuit was $193 \mathrm{kHz}$ which is about the expected adequate frequency of through heating of the filament to semi solid temperature (see Table 14, chapter 4.1.3). This was connected to an adjustable power source.

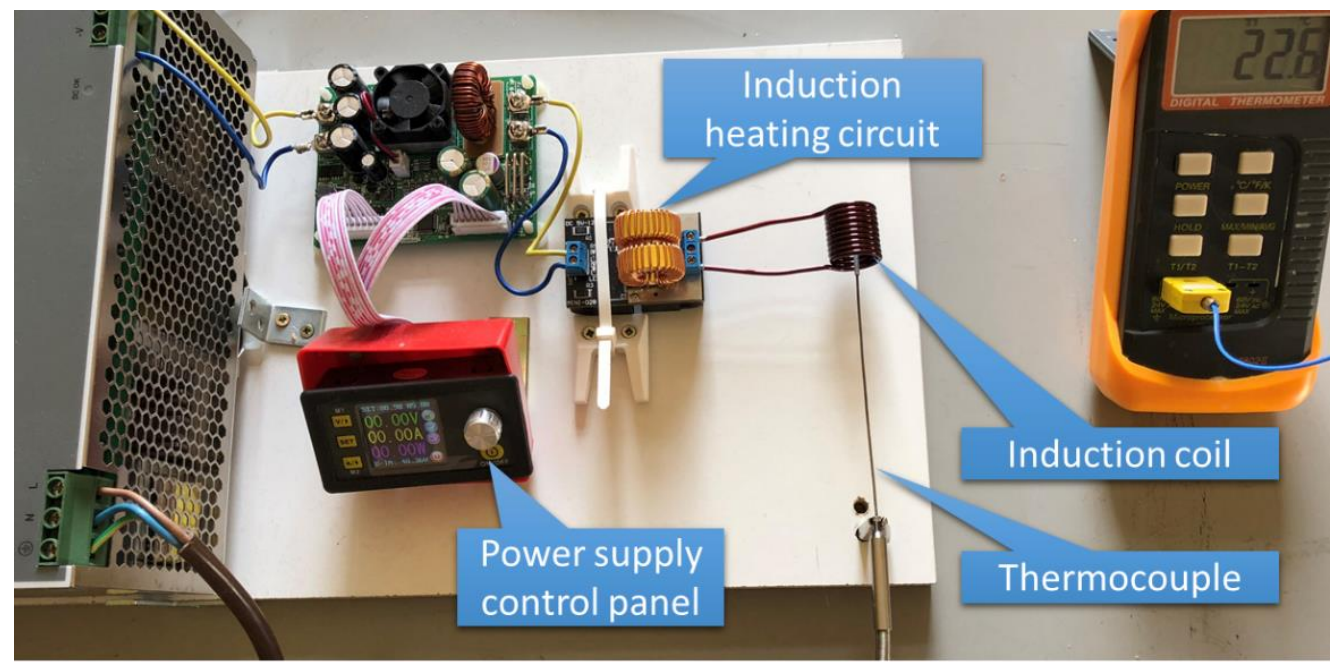

Figure 125 - Small scale induction heating experiment setup 
For that experiment several $10 \mathrm{~mm}$ filament samples (see Figure $126 \mathrm{left}$ ) have been prepared with a drilling of $1 \mathrm{~mm}$ diameter and $5 \mathrm{~mm}$ depth in the center to insert the thermocouple (see Figure 126 right).

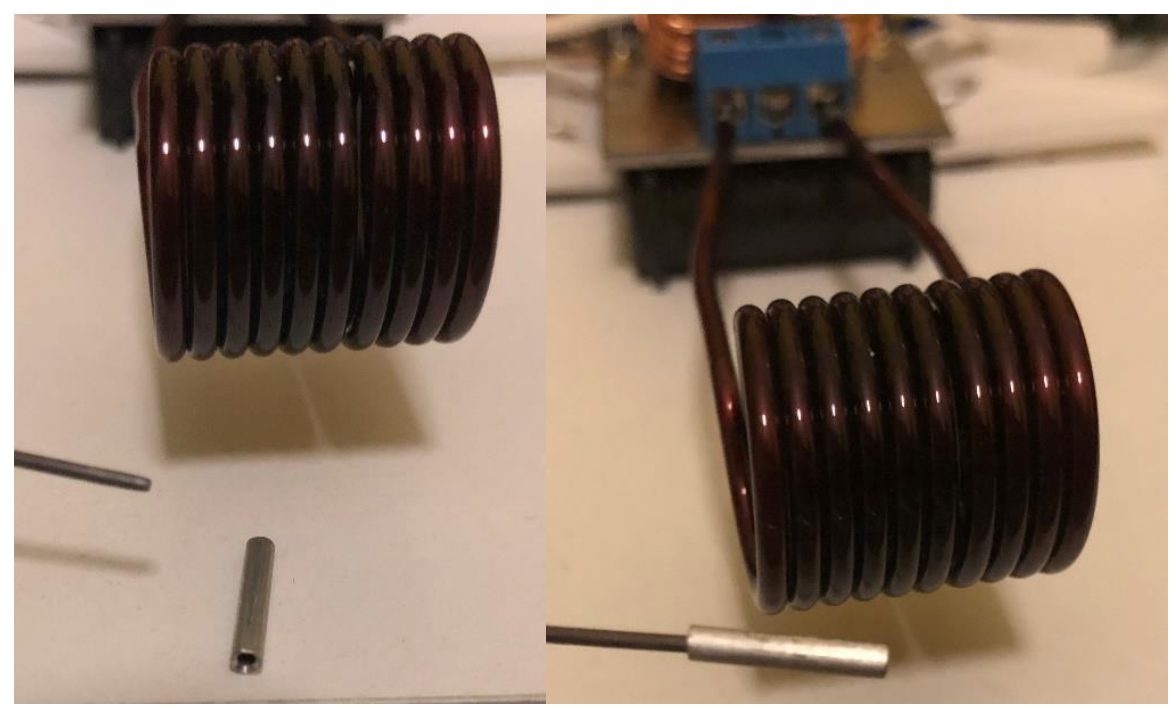

Figure 126 - Left) filament sample with drilling right) filament fitted on thermocouple Initial heating attempts (see Figure 127) show that depending on the voltage and position, temperatures up to $350^{\circ} \mathrm{C}$ can be reached with a very simple setup.

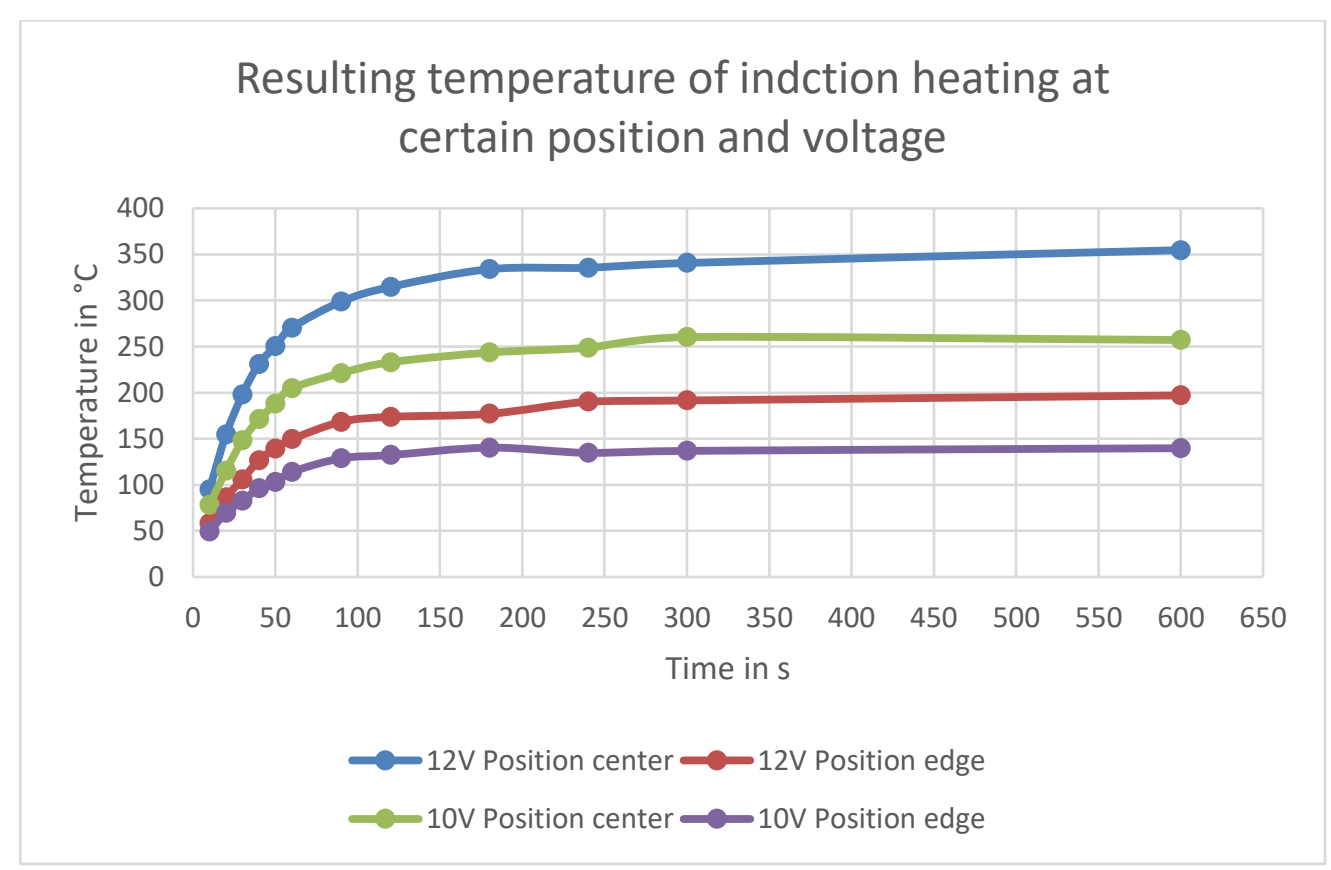

Figure 127 - Initial induction heating temperature results 
Based on that a simple experiment with 4 replicates has been designed and completed to understand the temperature that can be reached at two positions (center/edge) in the work coil as well as 2 voltages $(10 \mathrm{~V} / 12 \mathrm{~V})$. The maximum temperature was determined after $40 \mathrm{~s}$ which would reflect $0.5 \mathrm{~mm} / \mathrm{s}^{24}$ filament velocity which is the same as chosen for the induction heating simulation.

The resulting temperature show very little standard deviation (about $2^{\circ} \mathrm{C}$ center and about $6^{\circ} \mathrm{C}$ at the edge) and can reach up to $225^{\circ} \mathrm{C}$ in the center position at $12 \mathrm{~V}$ (see Figure 128).

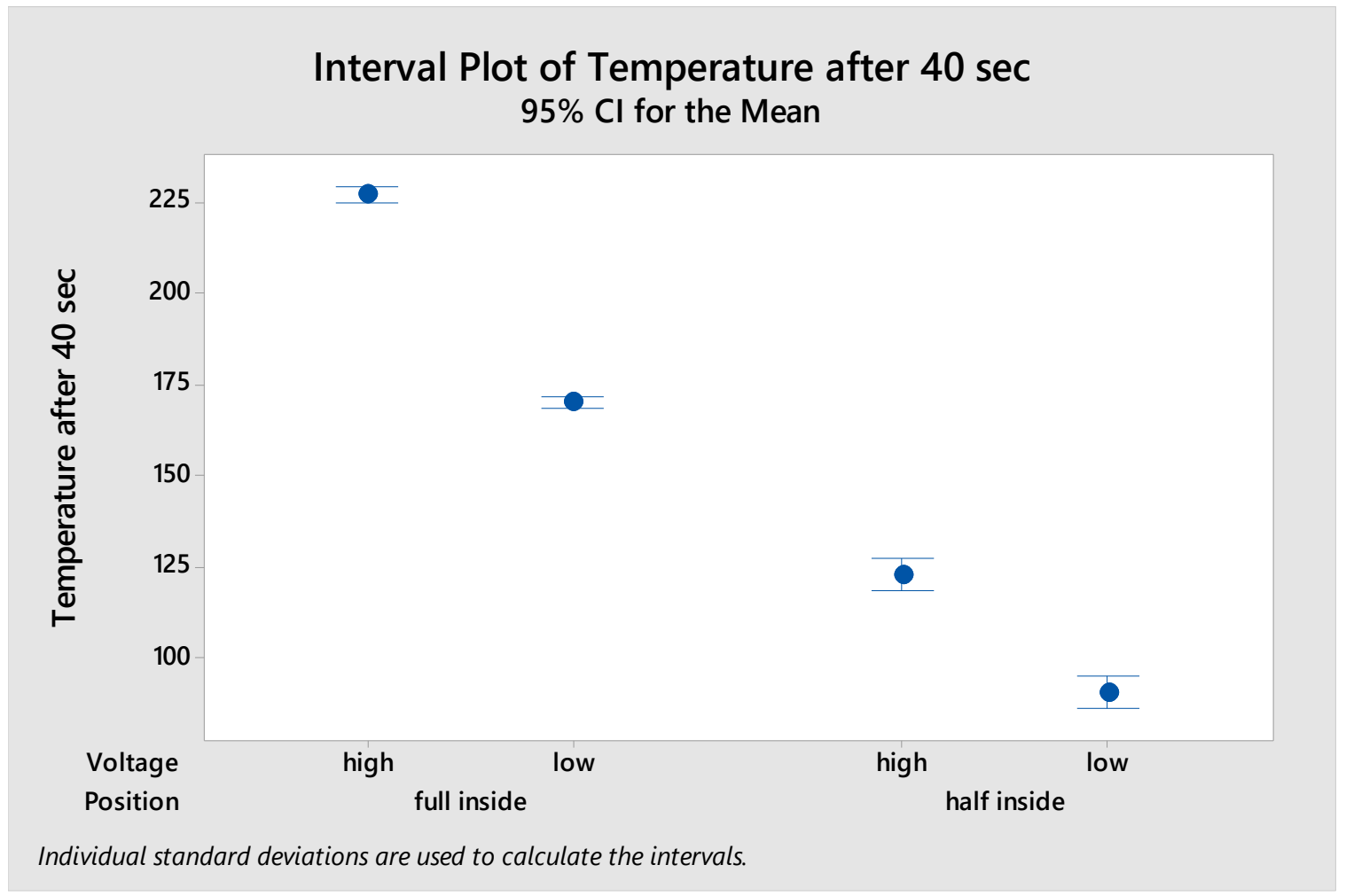

Figure 128 - Interval plot of temperature after 40 seconds

\footnotetext{
${ }^{24}$ Note: the experiment does not reflect an actual movement through the nozzle such as the temperature reached after 40 s processing the filament at $0.5 \mathrm{~mm} / \mathrm{s}$ would be different than the static holding at center or edge. As this is just a proof of concept experiment this simplification is deem adequate.
} 


\section{RESULTS AND DISCUSSION}

\subsection{Initial Al 4018 filament experiments}

Initial experiments showed the importance of precise composition of the material. A deviation in Si composition (-2.7\%) as seen in Al 4018 wire resulted in about $25^{\circ} \mathrm{C}$ higher temperature to achieve $50 \%$ fraction solid (see Figure 63 ). Standard wire drawing processes as used by Elisental do result in a loss of dendritic or globular microstructure (Figure 55). In contrast the wire drawing process allows as a severe plastic deformation (see chapter 2.2.3) and the formation of fine grain globular material. Due to the deviation in material (Si and Ag) the Al 4018 wire has been declined as a future experiment material.

\subsection{Material production}

It was noted that the material produced for semi-solid casting does not immediately show globular structure (see Figure 72 ) but requires a defined step heating process before processing in a semi solid state. When processing the "semi-solid" ready raw material through a traditional extrusion process similar to the wire drawing process the existing dendritic microstructure is dissolved completely. As such the filament production process using magnetically stirred alloy does not add additional value to achieve a MEAM ready filament. More so the created filament must be further processed through heat treatment in the experiment extrusion setup to achieve the semi-solid extrusion readiness.

A final MEAM process filament must be created to a direct globular structure which is currently not commercially available. It is expected to use a casting style production of filament. Rheocasting (stirring) or severe plastic deformation combined with reheating and casting are the best approaches leading to a globular and minimized grain size.

\subsection{Proof of concept experiments}

The initial use of A356, THIXALLOY 540 and Al 4018 without understanding of its microstructure, adhesion to the nozzle, temperature requirements and time-based changes in microstructure do not allow an extrusion process. It is required to 
establish the optimal development to a globular microstructure as well as understand the aging (time-based coarsening) of the microstructure.

While the acquired gantry system, wire feeder and inert gas chamber were not used or useful, the resistive heating nozzle was capable of performing within the temperature ranges very well. Especially the maximum setting of $650^{\circ} \mathrm{C}$ at the nozzle orifice area could be reached without any problems.

\subsection{Simulations}

\subsubsection{Simulation of heated channel dimension changes}

Overall the changes in dimension (see max displacement vector in Table 26) must be considered for the insulation and mount of the nozzle (as also seen in Figure 77). From the change of the orifice (about $1 \%$ ) no significant changes in the backextrusion behavior is expected. The influence would be more expected from the difference of the filament diameter and the heating channel (used nozzle $+0.1 \mathrm{~mm}$ ) than the dimension changes due to heat expansion. Even with future changes of the nozzle for aluminum semi solid temperature ranges the orifice dimension change can be neglected.

Table 26 - Dimension change of nozzle at $585^{\circ} \mathrm{C}$

\begin{tabular}{|c|c|c|}
\hline $\begin{array}{c}\text { Change of nozzle } \\
\text { orifice }\end{array}$ & $\begin{array}{c}\text { Change of nozzle } \\
\text { orifice }\end{array}$ & $\begin{array}{c}\text { Max displacement } \\
\text { vector }\end{array}$ \\
\hline $0.02 \mathrm{~mm}$ & $1 \%$ & $0.254 \mathrm{~mm}$ \\
\hline
\end{tabular}

\subsubsection{Simulation of resistive heating}

The simulation results with the adapted nozzle temperature distribution (see Figure 129) showed the significance of an appropriate nozzle design with a uniform temperature distribution especially near the nozzle outlet. With the current nozzle design a semi-solid temperature range (achieving about $50 \%$ liquid fraction) cannot be achieved without significant overheating near the center of the nozzle channel. Besides the microstructure and adhesion of the filament this drop to about $20 \%$ liquid at the nozzle outlet would have led to clogging. In case the extrusion is 
still successful unwanted microstructure changes may occur or too little heat energy is left for layer bonding with prior extruded material.
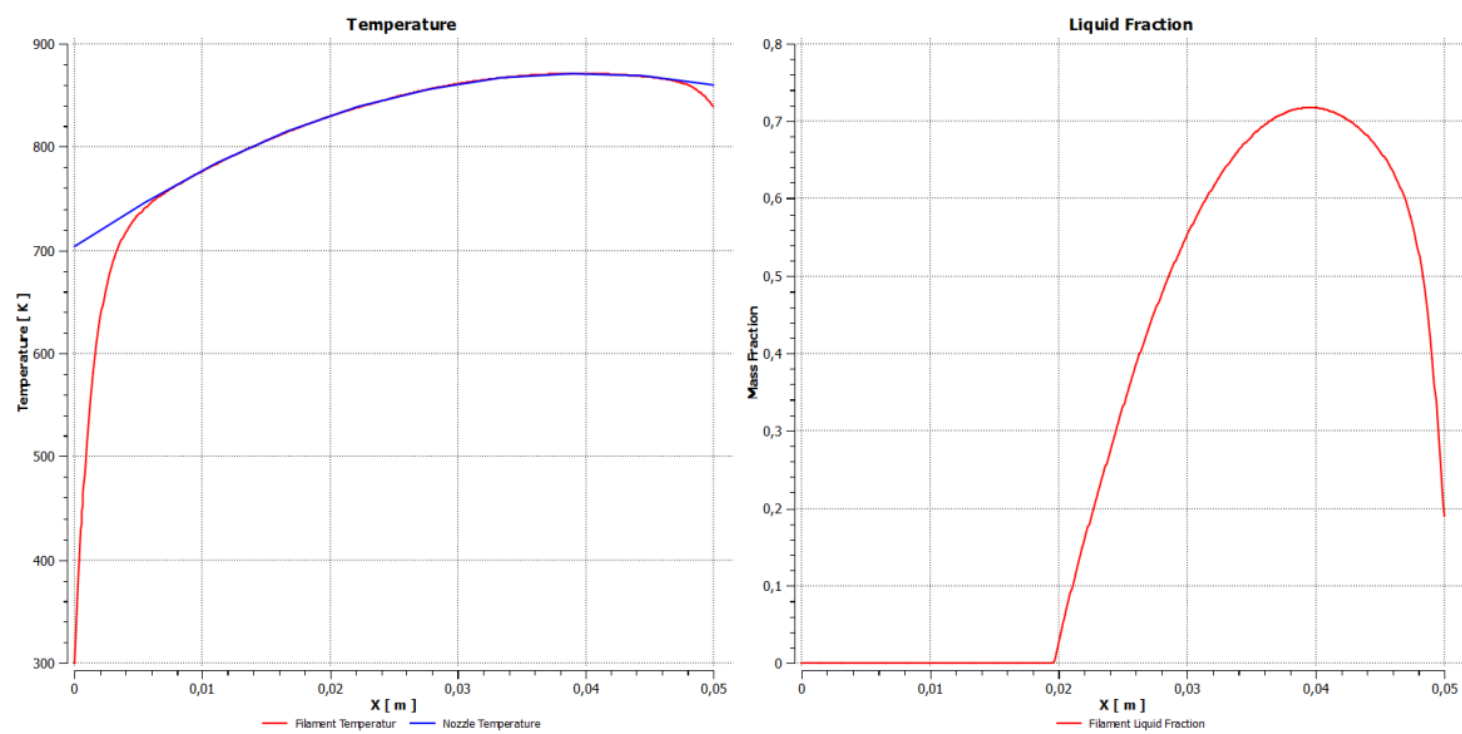

Figure 129 - Adapted Simulation Results for A356 heating with nozzle temperature profile diagram for resulting temperature and mass fraction liquid with setting $585^{\circ} \mathrm{C} / 858 \mathrm{~K}$

An experiment design to review the microstructure development of the filament needs to be adapted using the outcome of the simulation.

For the design of the operational MEAM apparatus a multi circuit resistive heating is needed to overcome the temperature distribution within the nozzle. Especially at the area of the semi solid material the nozzle temperature needs to be within the $\pm 3^{\circ} \mathrm{C}$ range or less. It should be reviewed whether it is advisable to slightly design a higher temperature at the nozzle outlet to overcome the convection as well as aid the layer bonding.

\subsubsection{Simulation of inductive heating}

Overall the simulation shows good results in achieving the required range of liquid fraction (see Figure 130 right). Also, the cross section of the filament shows adequate through heating with a maximum difference of about $1.5^{\circ} \mathrm{C}$. While those results are very satisfactory the sensitivity of the excitation vs the liquid mass fraction as shown in Figure 131 are of concern. Any object within the electromagnetic field would change the excitation drawn and the ohmic losses significantly so that it need to be assumed a constant temperature range of about 
$\pm 3^{\circ} \mathrm{C}$ cannot be achieved. This does correlate to the results of Garcia [114] testing such a filament main heating while failing to keep a constant temperature.
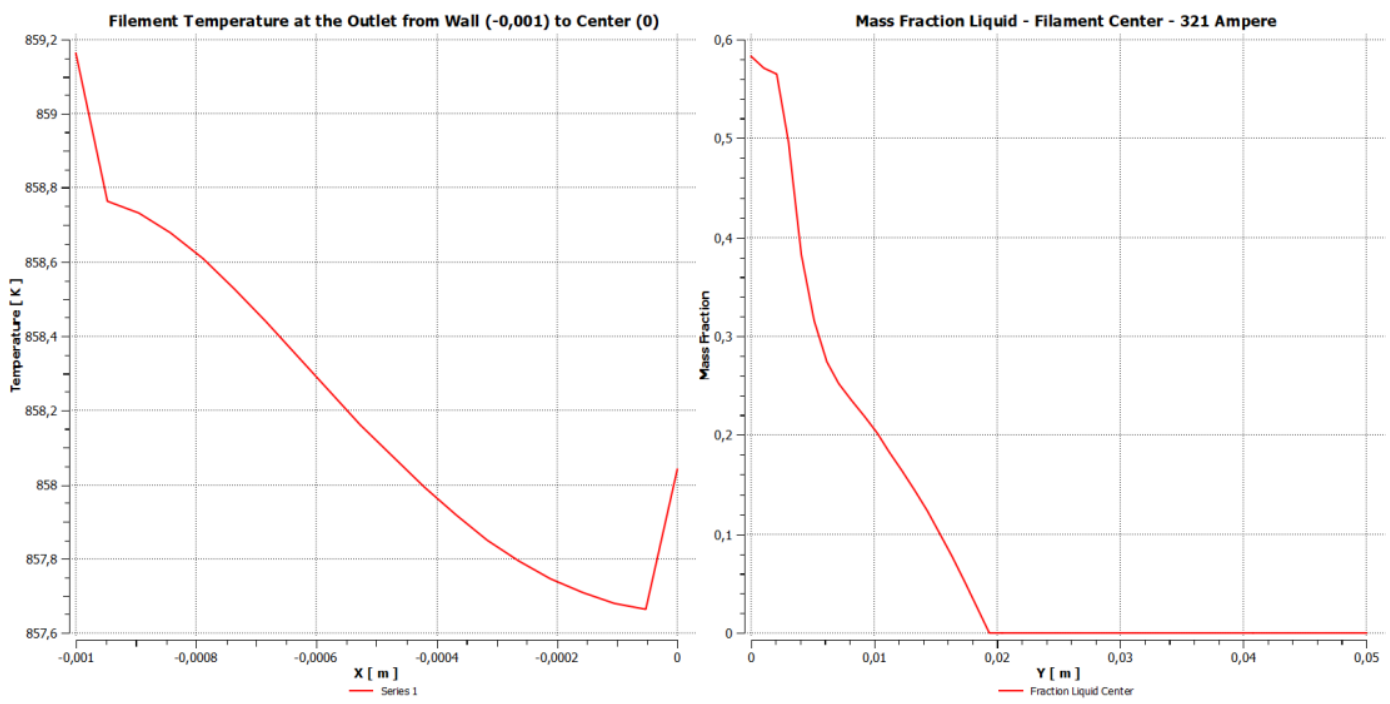

Figure 130 - Induction heating simulation results - left) Outlet temperature difference Wall -Center for 318A right) Mass Fraction Liquid at the center line 312A

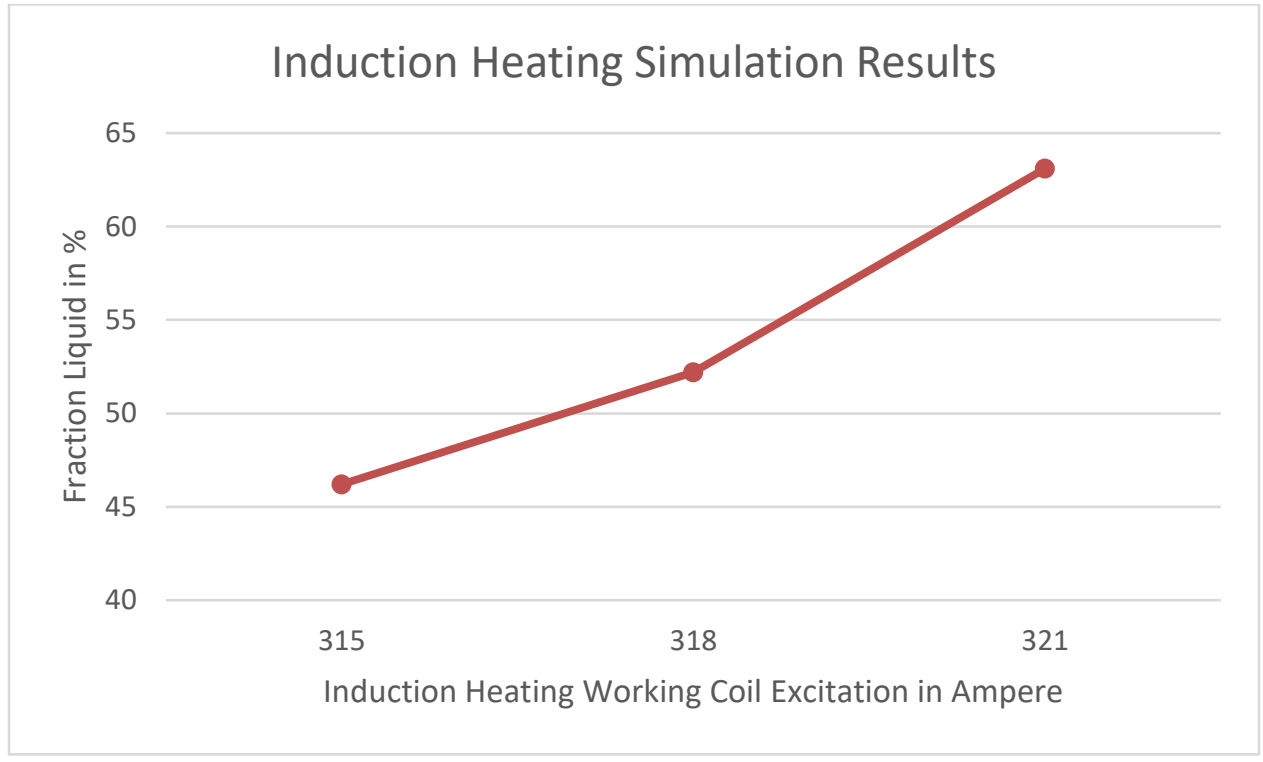

Figure 131 - - Induction heating simulation result / sensitivity of the excitation vs. fraction liquid

Thus, induction heating as the final filament heating to the semi solid state is not recommended. While the (in this work) tested resistive heating nozzle needs to be improved, the work of Chen et al. [113] show good temperature control with a 
multiple resistive heating circuits solution. To allow a minimized area of temperature that cannot be fast increased and decreased (i.e. the resistive area) a pre-heating before the resistive heating nozzle is advised. Such pre-heating does not need the temperature precision as the area with the semi-solid alloy. It might be also in a position of the MEAM apparatus that can be more easily shielded and controlled with respect to other objects moving within the electromagnetic field.

\subsubsection{Simulation summary}

Overall the simulation shows that the nozzle dimension changes at the semi-solid temperature can be neglected. The induction heating is not recommended for a heating of the filament within the semi solid range but is potentially adequate as a filament pre-heating to aid and shorten a resistive heating nozzle. The resistive heating nozzle needs to have at least a 2-circuit heating design to manage the heating up to the semi solid range as well as keeping that semi-solid mass fraction in an adequate range at the nozzle neck and outlet.

\subsection{Experiments}

\subsubsection{Heating to globular microstructure experiment}

The microstructure measured by the RQI is affected by the hold time and temperature for A356 (see Figure 132 and Figure 133). For THIXALLOY it is only affected by the holding time (see Figure 135).

The Grain Size for A356 is only dependent on the holding time (see Figure 134) while for THIXALLOY 540 it is dependent on both holding time and temperature (see Figure 136).

The fraction solid values achieved are not dependent on the temperature or holding time. It would have been expected that the temperature has an effect as defined in section 2.1.3. But due to the experimental nozzle the material must pass through an area of lower temperature before quenching. It seems the effect of that area and the time required for manual pushing into the water filled test tube diminishes that effect. 


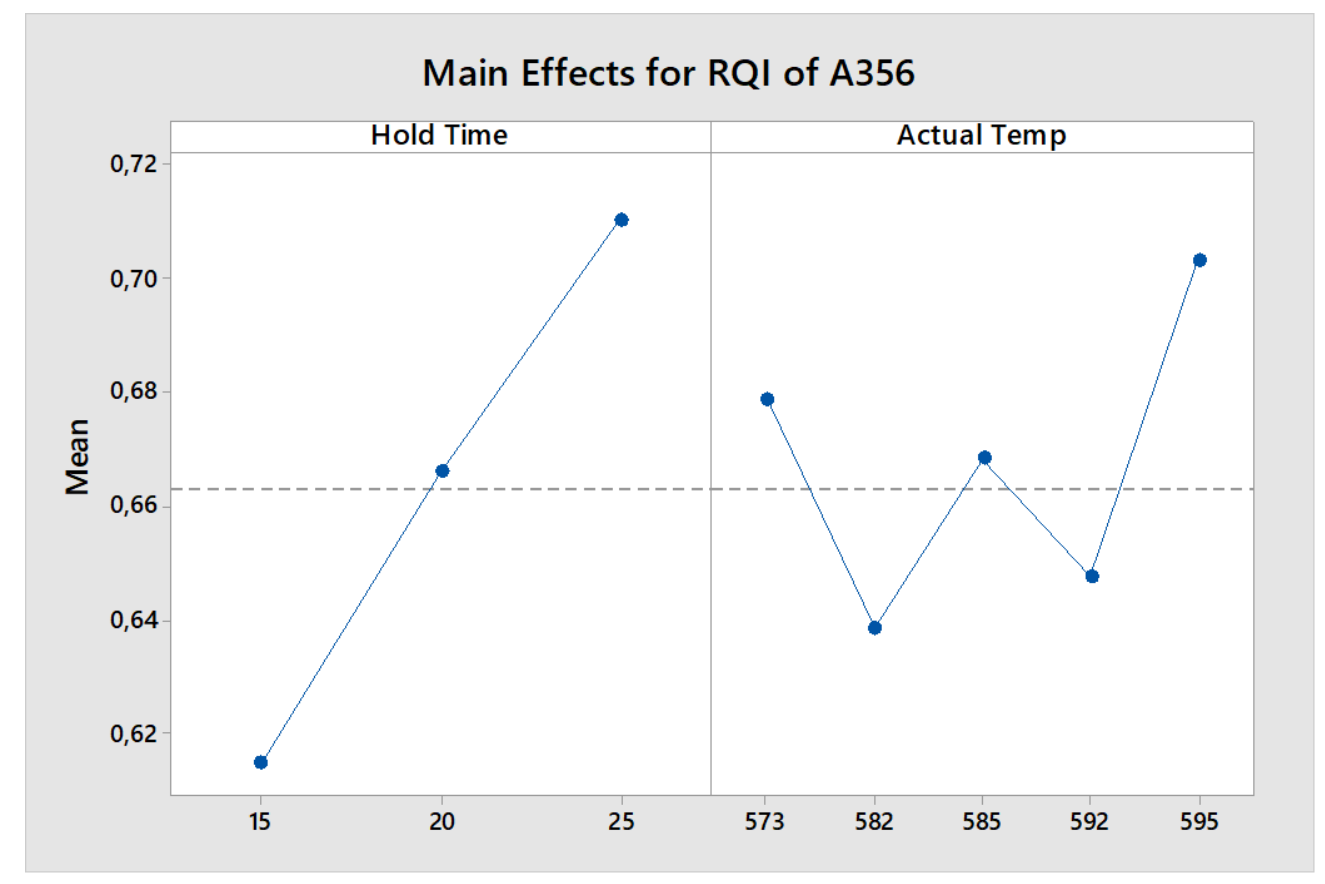

Figure 132 - A356 main effects of temperature (in ${ }^{\circ} \mathrm{C}$ ) and hold time (in min) on RQI

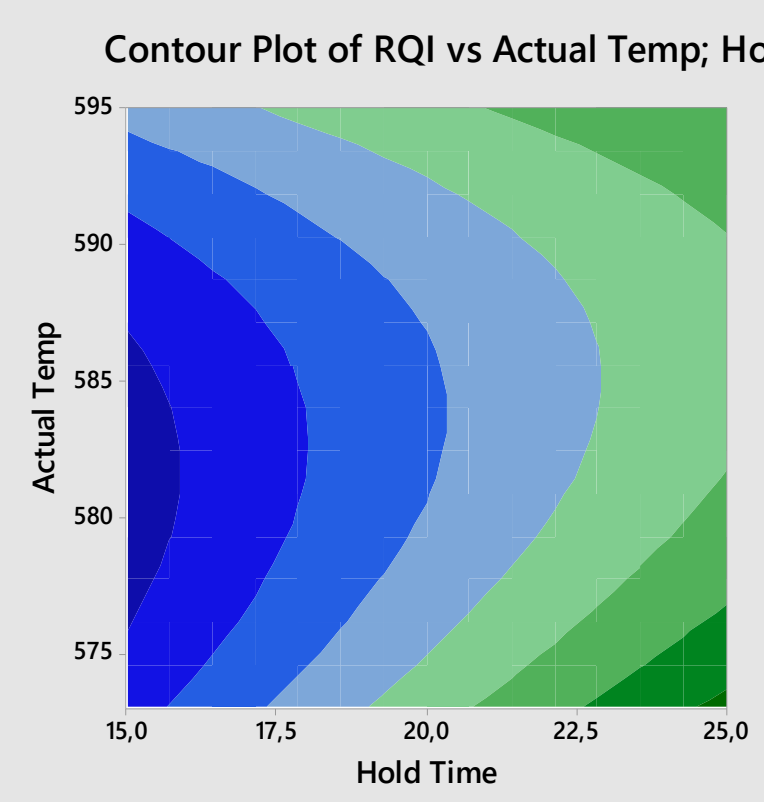

Figure 133 - A356 contour plot of temperature and hold time (in min) on RQI 


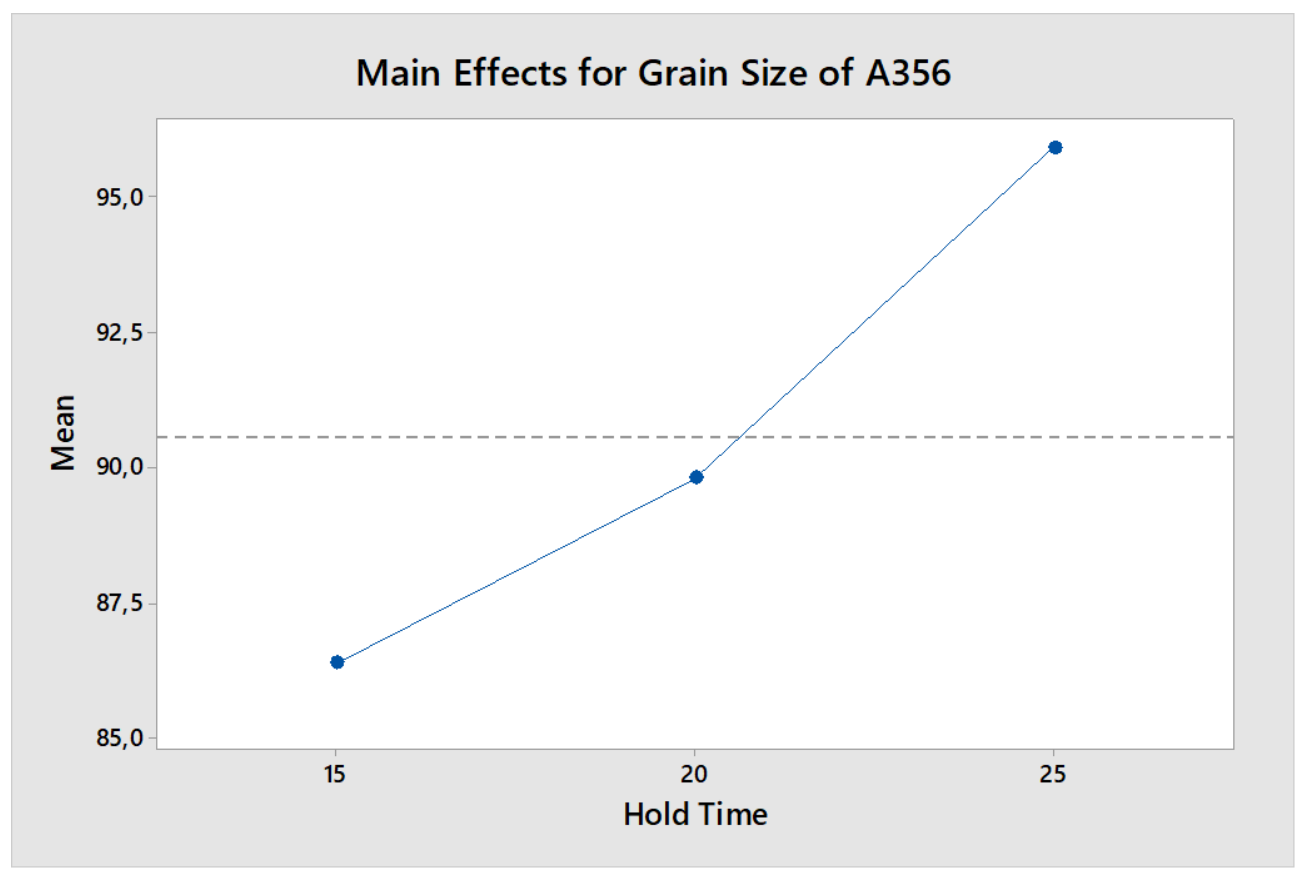

Figure 134 - A356 Main effects of hold time (in min) on grain size

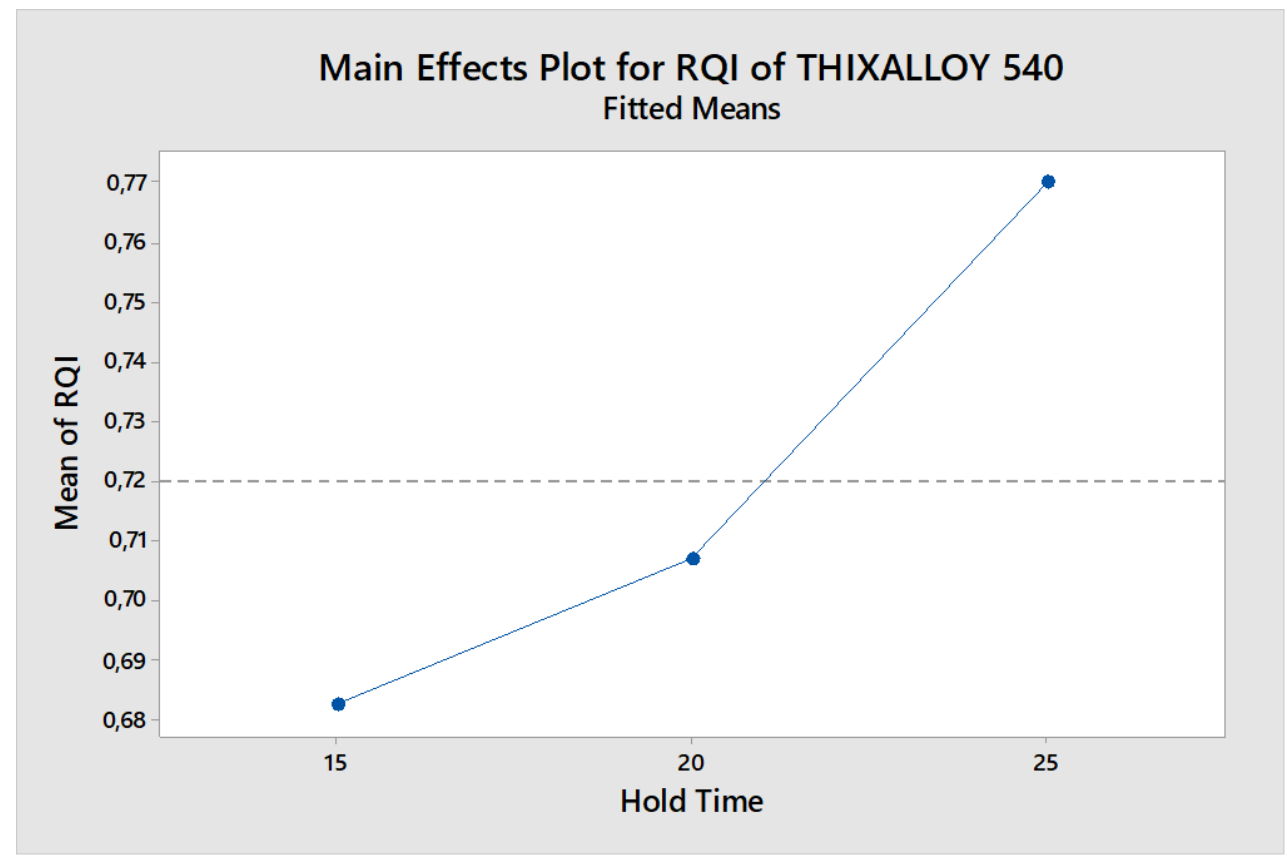

Figure 135 - THIXALLOY 540 Main effects of hold time (in min) on RQI 


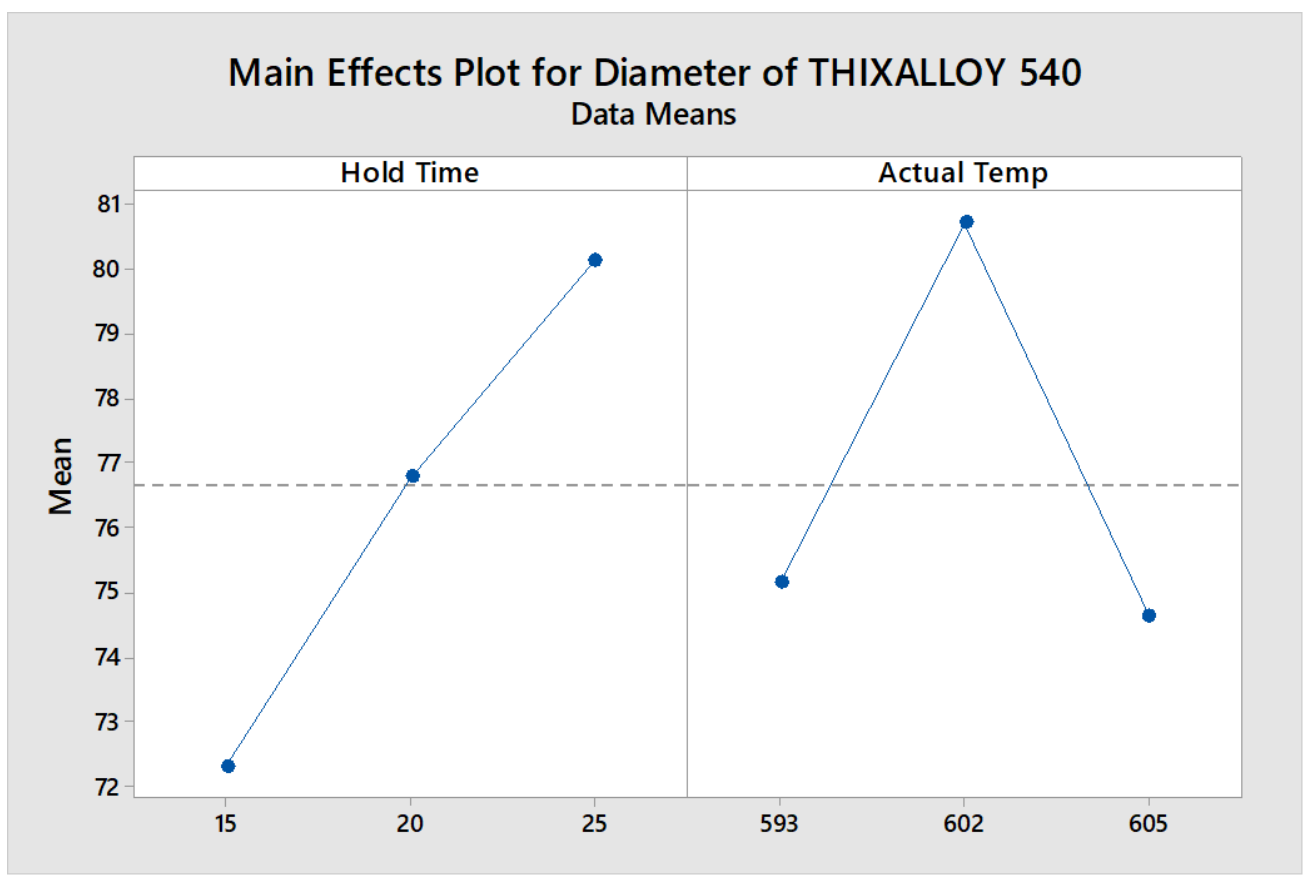

Figure 136 - THIXALLOY 540 Main effects of hold time and temperature on grain size

The goal of this experiment was to identify the parameter for a successful (see failure proof of concept in section 3.4) extrusion. The Tukey Test below supports the parameter choice.

\section{Comparisons for RQI}

Tukey Pairwise Comparisons: Hold Time

Grouping Information Using the Tukey Method and 95\% Confidence

\begin{tabular}{lrrrr} 
Hold & & & & \\
Time & N & Mean & Grouping \\
\hline 25 & 20 & 0.715417 & A & \\
20 & 22 & 0.667750 & A & B \\
15 & 21 & 0.622342 & & B
\end{tabular}

Means that do not share a letter are significantly different.

\section{Tukey Pairwise Comparisons: Actual Temp}

Grouping Information Using the Tukey Method and 95\% Confidence

\begin{tabular}{lrrrr}
$\begin{array}{l}\text { Actual } \\
\text { Temp }\end{array}$ & N & Mean & Grouping \\
\hline 595 & 12 & 0.703040 & A & \\
573 & 8 & 0.683618 & A & B \\
585 & 11 & 0.674074 & A & B
\end{tabular}


$\begin{array}{lllll}592 & 12 & 0.647529 & \text { A } & \text { B }\end{array}$

$\begin{array}{llll}582 & 20 & 0.634254 & B\end{array}$

Means that do not share a letter are significantly different.

Based on the results the factor levels for the extrusion experiment are chosen as follows:

- A356 Temperature $-595^{\circ} \mathbf{C}$ for higher fraction solid (please see also the respective process window from Finke [71]/Figure 46 expecting $F_{s}>0.55$ )

- A356 hold time - 25 min for a higher Rheocast Quality Index (RQI). 25 min also corresponds with a larger grain size (average up to $96 \mu \mathrm{m}$ ) but with an initial extrusion outlet of planned $1.1 \mathrm{~mm}$ the threshold defined by Finke [71] (6x of average grain size) is not exceeded by the material.

- THIXALLOY 540 hold time - $\mathbf{2 5}$ min while the temperature is kept at the tested $605^{\circ} \mathrm{C}$.

The expected average values for the RQI and Grain Size are shown in Table 27.

Table 27 - Expected RQI and Grain Size for holding time of 25 min prior extrusion

\begin{tabular}{|l|c|c|}
\hline Alloy & Average RQI expected & Average Grain Size expected \\
\hline A356 & $0.731 \pm 0.01\left(@ 695^{\circ} \mathrm{C}\right)$ & $97.2 \pm 3.9 \mu \mathrm{m}\left(@ 595^{\circ} \mathrm{C}\right)$ \\
\hline THIXALLOY 540 & $0.762 \pm 0.022\left(@ 605^{\circ} \mathrm{C}\right)$ & $78.1 \pm 2.6 \mu \mathrm{m}\left(@ 605^{\circ} \mathrm{C}\right)$ \\
\hline
\end{tabular}

\subsubsection{Aging experiment}

The aging experiment showed a growing but also stabilizing $R Q I$ as expected. This would not limit the processing of the material itself. Increasing precipitation could be observed within the globular grains. The grain size is growing within the measured time by about $0.75 \mu \mathrm{m}$ per minute holding time (approximation based on A356 $-0.72 \mu \mathrm{m} / \mathrm{min}$ and THIXALLOY $540-0.71 \mu \mathrm{m} / \mathrm{min}$ ). As the initial grain size is dependent on the material history or filament production it is assumed for a model that the grain size starts with an adequate one for semi-solid processing. Based on the slope of grain growth and expected initial semi solid grain size a 
prediction model has been built showing the maximum holding time until the limit of outlet orifice (6x average grain size [71]) has been reached.

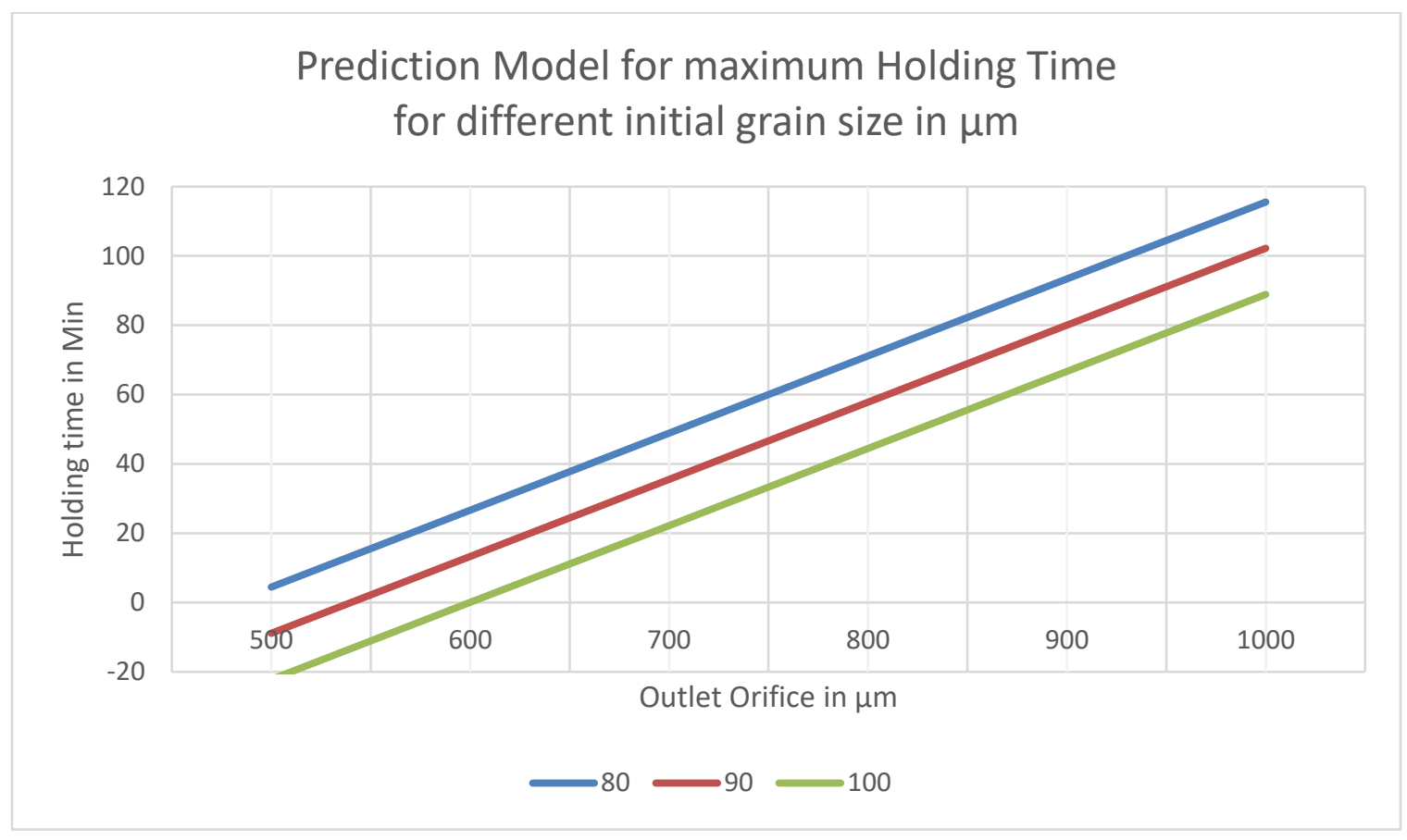

Figure 137 - Holding time prediction model for A356

This model shows that for some combination such as $100 \mu \mathrm{m}$ starting grain size the limit has already been reached for $0.5 \mathrm{~mm}$ with no holding time i.e. negative holding time. The model would need to be validated for times larger than $50 \mathrm{~min}$ but those would not be practical holding times needed for an MEAM apparatus. Those have been included to show potential holding times for outlet orifices up to $1 \mathrm{~mm}$.

For the extrusion experiment an outlet orifice of $1.1 \mathrm{~mm}$ has been chosen also to remove the influence of the grain size for clogging.

\subsubsection{Cleaning process development}

The successful cleaning process of the nozzle is determined as a chemical cleaning after removing of larger remains of the alloy material. The heating nozzle material as well as the resistive heating coil was chemically resistant against the $50 \%$ sodium hydroxide solution. Even after more than 100 cleaning cycle iterations both tested nozzles do not show any degradation. The applied boron-nitride 
coating was also removed during the cleaning which is not desirable. It is assumed that a permanent coating as applied to extrusion dies through Chemical vapor deposition (CVD) will not be affected by the cleaning process. It needs to be reviewed how the major contamination of alloy material can be removed without mechanically affecting such coating. The cleaning process with sodium hydroxide solution will take 10-15 min with dimension similar to the current experimental nozzle $(5 \mathrm{~cm}$ long) when combined with ultrasonic agitation. A soaking process needs to be applied after cleaning to remove the sodium hydroxide and the generated sodium and aluminum salt.

\subsubsection{Extrusion experiment}

For the final extrusion experiments all samples showed successful extrusion for both A356 (see example Figure 138 and appendix 0) and THIXALLOY 540 (see appendix 0). Only one sampled clogged (see Figure 139) during the sample attempt due to operator error (only first temperature increase was applied). After correcting the error i.e. immediate increase by $15^{\circ} \mathrm{C}$ and slightly more force the sample was extruded.

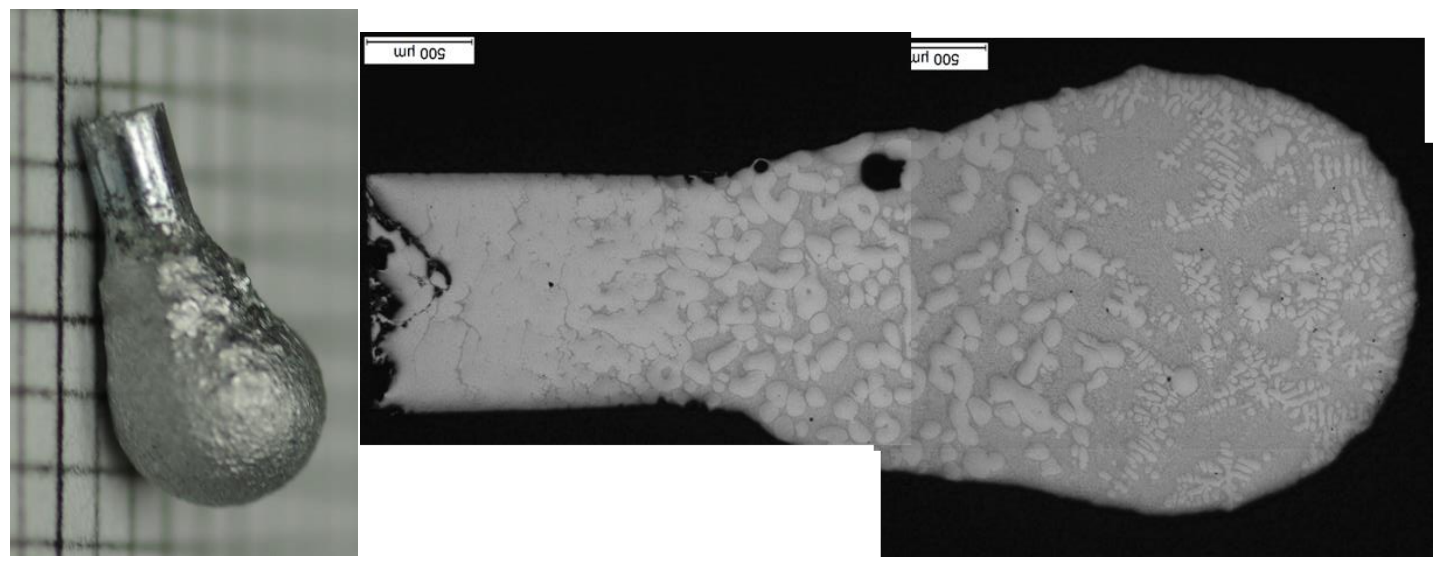

Figure 138 - A356 extrusion run 5 - left) resulting sample right) microstructure 


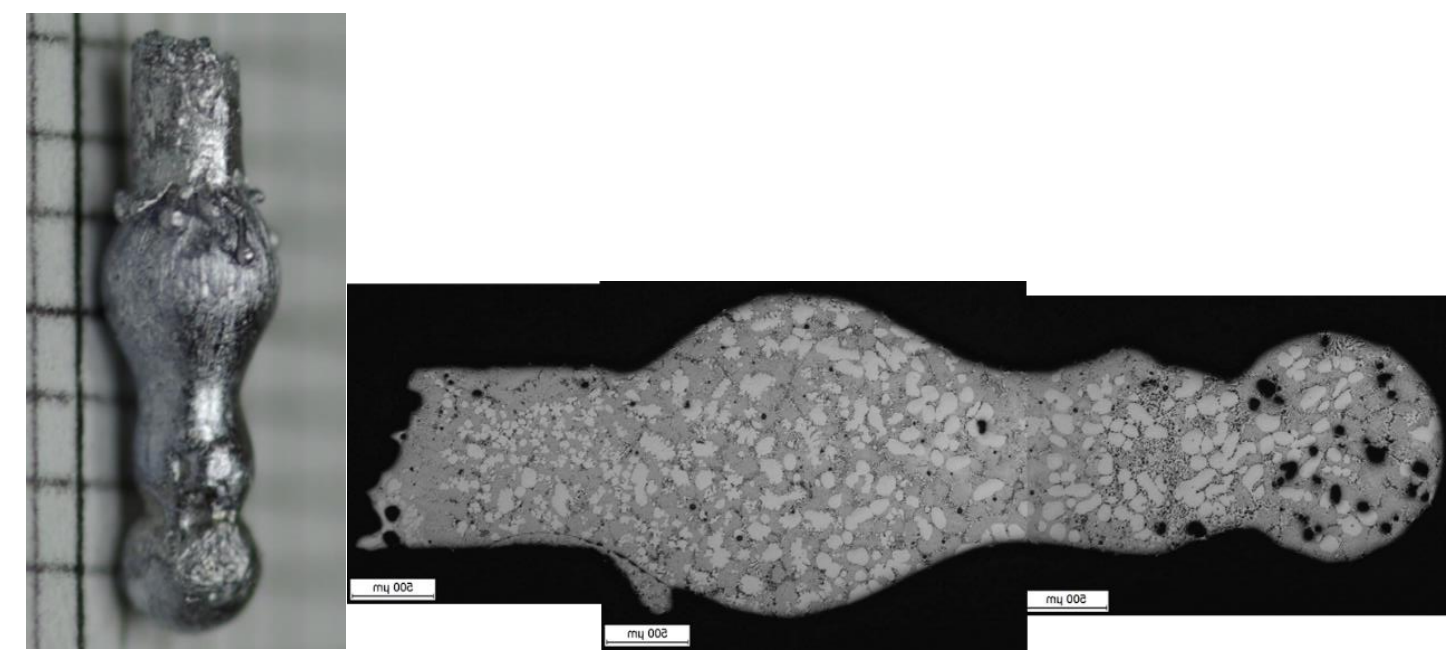

Figure 139 - THIXALLOY 540 run9 - left) resulting sample right) microstructure

All samples showed significant segregation of liquid and solid fraction with extruding first the liquid state followed by a compressed and deformed solid state. Overall some THIXALLOY 540 showed slightly better results (see sample 3-6 appendix 0) i.e. less segregation. The sample 9 for THIXALLOY 540 (see Figure 139 right) resulted in only minimal segregation. Due to the operator error the extrusion first clogged and a solid skin developed at the already extruded bubble. The additional force then extruded the sample after temperature increase. It is assumed that the higher force applied and the solid skin operating at a plug for at least some time resulted in a force / velocity combination that results in a successful extrusion without segregation.

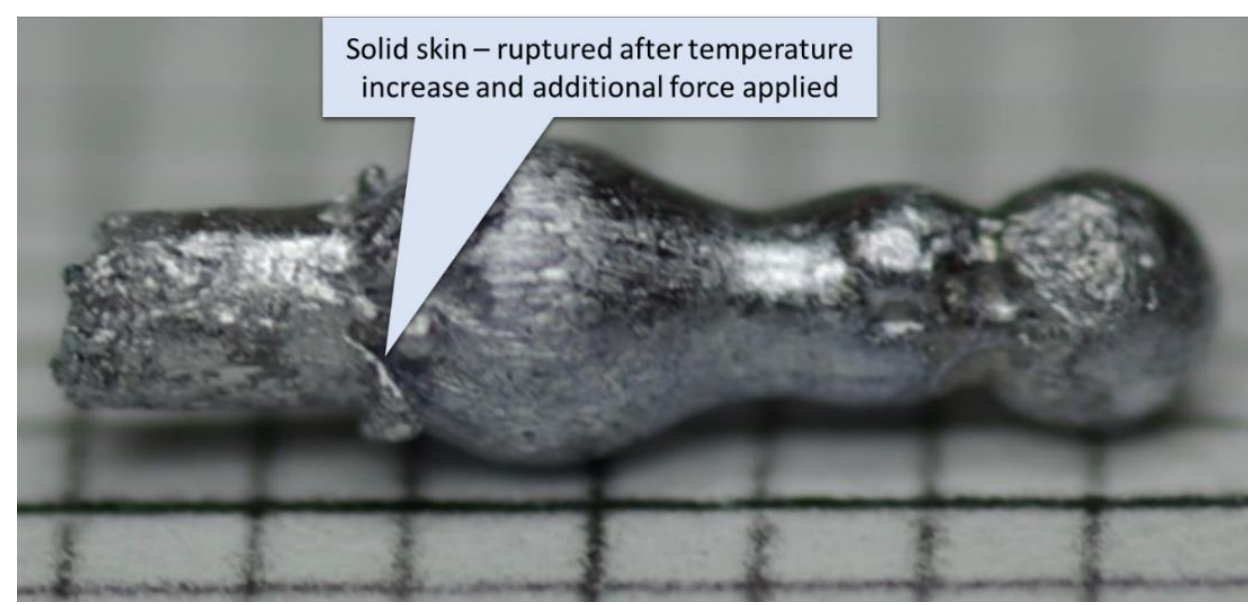

Figure 140 - THIXALLOY 540 run 9 extrusion example with ruptured skin 
The results do correlate with the segregation and threshold force for nonsegregation extrusion shown by Finke [71]. As discussed in section 2.4.3 and shown by Figure 46 as well as Figure 48 there is a process window that requires a defined minimal force and velocity. Finke also described that there is a start stop phenomena that may lead to segregation.

With the current nozzle restrictions of non-uniform temperature and no enduring coating as well as the filament not directly ready for extrusion in a globular semi solid state further experiments identifying the force / velocity process window are not possible. Also, whether start / stop phenomena can be avoided or needs to be overcome cannot be investigated.

Still the current experiments show that MEAM extrusion of aluminum alloy with a respective high RQI and small grain size is possible within the adequate process window of semi-solid state.

\subsubsection{Induction Pre-Heating experiment}

The induction pre-heating experiment results show a very easy process to preheat the filament to about half of the semi-solid temperature. This can be used to reduce the size of the subsequent resistive heating nozzle. That setup would allow to restrict the material in semi solid state during temperature elevated holding times as well as require far less accuracy and power of the induction heating setup. For a MEAM apparatus the power needs to be increased to process at higher speed than tested in the experiment.

\subsubsection{Experiment summary}

The experiments show that the RQI reaches $72-75$ for a non-globular filament after about $25 \mathrm{~min}$. This is assumed to be adequate for semi solid processing. The aging results show a significant increase of globular size up to $100 \mu \mathrm{m}-110 \mu \mathrm{m}$ after $50 \mathrm{~min}$ as well as a creation of precipitates within the grains. This grain growth limits the holding time depending on the nozzle outlet orifice as well as the starting grain size (e.g. about $20 \mathrm{~min}$ for $100 \mu \mathrm{m}$ starting grain size with $0.7 \mathrm{~mm}$ outlet orifice). A model has been developed to estimate the maximum holding time. The extrusion 
experiments showed it is possible to repeatedly extrude the semi-solid material. On the contrary only one sample did not showed segregation within the resulting extruded material. All other samples showed significant segregation while THIXALLOY 540 had slightly better outcome. It is assumed that the adequate velocity / force window cannot be reached with the existing nozzle and manual processing. Due to the filament material and the inadequate temperature distribution an automated feeding is not possible as of now.

An ultra-sonic improved cleaning process has been used during the final extrusion experiments. That does result in a faster completion of the process i.e. only 10$15 \mathrm{~min}$ needed. Reduced adhesion can be achieved by coating the inner wall of the nozzle channel and neck with Boron-Nitride.

Finally, a proof of concept of an induction pre-heating showed a low-cost option to reach about $50 \%$ of the semi-solid temperature range. 


\section{MEAM PROCESS DESIGN}

Based on the experiments the following filament, machine and processing properties as well as design elements are proposed to create an operational MEAM process using semi-solid aluminum alloy.

\subsection{MEAM feedstock material and shape design}

It has been shown during this work that drawing or extruding aluminum filament is not adequate to achieve an immediately semi-solid processing ready material.

Overall the small scale MEAM process compared to casting of aluminum billets require narrower parameter ranges. The behavior in the nozzle for example the fraction liquid is dependent on the raw material composition. As for A356 the allowed composition ranges (e.g. Si 6.5\% - 7.5\%) would influence the processability significantly (see section 2.1.9 and Figure 11). For the raw material in the MEAM process the composition ranges should be reduced e.g. $6.8 \%-7.2 \%$. For direct processing of aluminum alloy, it is critical that the material loaded into the apparatus shows already a processing ready globular structure. This will ensure that the material can be heated into the semi-solid state for a very brief time right before extrusion. As such the holding time needs to be kept to a bare minimum also to reduce the coarsening of the microstructure or other changes such as developing precipitates. The development of the globular microstructure (for maximizing the RQI) within the MEAM apparatus is deemed impractical as there has been no process found that would speed up this below 10-15 minutes. E.g. this would require maintaining material at the elevated temperature for $>15 \mathrm{~min}$ of extrusion. Production of the raw material need also to ensure that porosity is reduced to a minimum. The grain size should be as small as possible for products with fine details e.g. wall size $<2 \mathrm{~mm}$. For extrusion of $0.5 \mathrm{~mm}$ material the average grain size needs to be $<80 \mu \mathrm{m}$ to adhere to Finke's [71] grain size $<1 / 6$ of extrusion diameter. It is expected that an ECAP (see 2.2.3 and Figure 1) processing followed by a semi-solid casting (see also [122] for thin wall casting) with immediate cooling of the filament would lead to adequate results. The grain size may be relaxed for production of larger parts without fine details. In such cases material with $100 \mu \mathrm{m}$ 
average grain size is sufficient which may reduce costs but also require a larger nozzle outlet orifice size $(>0.7 \mathrm{~mm})$. Production of such filament would for example not need an ECAP process and therefore reduce the cost. The extruded material needs to be free of an aluminum oxide layer to allow layer bonding. As such the filament needs to be free of any oxide layer already as removal of such layer in the MEAM apparatus (e.g. physical or with cleaning agent) is deemed impractical. For that the filament needs to be produced in an inert atmosphere or the oxide layer needs to be removed and after that the filament transported and stored in a protective atmosphere. Alternatively, a protective coating may be identified that protects the filament during the transportation and storage but dissolves during the heating process.

The diameter of the filament is an important design aspect as well. Larger diameter will result in rigid and therefore difficult to process filament. Any deformation of the filament may not be overcome by the filament feeder and may result in clogging at the beginning of the heating channel.

Using the above material processing and design constraints a feed stock is proposed and defined as Fused Bolt Fabrication (FBF). The concept is significantly different from a continuous coiled filament as the principle used is similar to the Stick deposition molding described in section 2.3.2.1. The raw material will be prepared as described above to ensure semi-solid ready microstructure and cast into round bolts with a male / female connection design at each end (see Figure 141). For feeding purposes the bolt should have a threaded structure at the outside. To allow positioning of the bolt a notch maybe required. This will keep each bolt oriented while the thread feeder is moving it into the heating nozzle and at a certain time allowing the next one to connect automatically. The channel prevents connected bolts from separating. The rectangular shape of the male connector allows the next bolt to be oriented correctly while supplied from a container to the channel. 


\section{Bolt Design \\ Male connector end}

Bolt Design

Female connector end
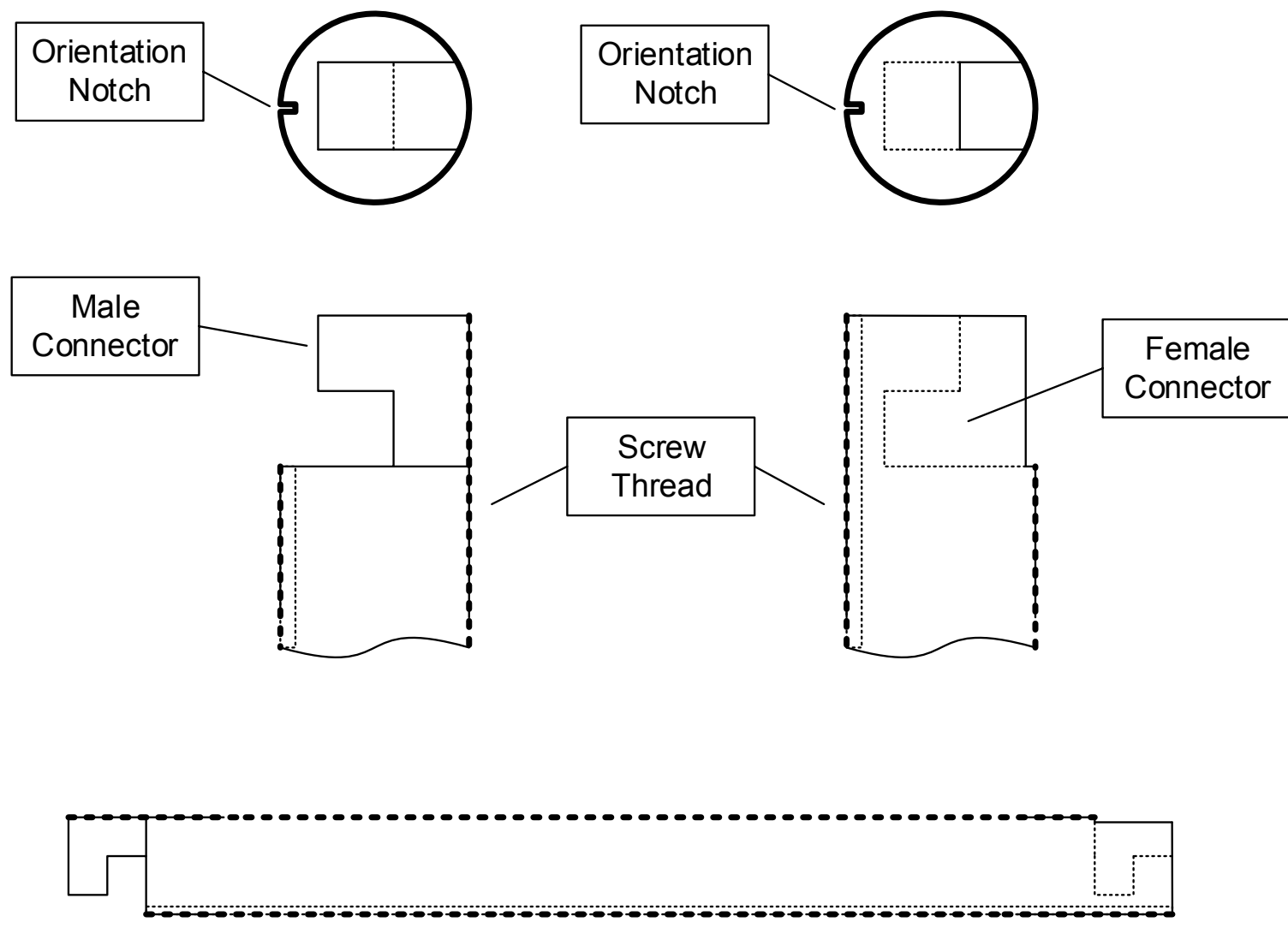

Figure 141 - Schematic Bolt design

With this design a set of bolts can be cast in a single step. It is suggested to store the aluminum oxide free bolts in a magazine either with a protective atmosphere or a protection coating. With an adequate bolt processing in the MEAM apparatus the rigidity of the bolt can be neglected. As such larger diameters such as $3-4 \mathrm{~mm}$ may be appropriate which would also allow a better semi-solid casting process.

Note: For further experiments the existing material could be used with a nozzle that has a uniform temperature at significant length of the heating channel, at the nozzle neck and outlet orifice. With such a nozzle batch processing would be possible so that verification of the processing parameter such as force vs. fraction liquid can be performed. 


\subsection{MEAM Nozzle and machine design}

The MEAM apparatus needs significant design changes to build a prototype for further experiments as well as for a final production ready apparatus.

\section{Nozzle}

The resistive nozzle, while in its current design does not show a constant temperature window, has still been identified as applicable for the MEAM process. Additional heating circuits especially at the nozzle tip are required for a constant $\left( \pm 2^{\circ} \mathrm{C}\right)$ temperature. It is assumed that 2 heating circuits may be sufficient when combining with an induction preheating. The resistive heating area for a production apparatus should be designed as small as possible to restrict the material aging (coarsening, precipitation) to a minimum during hold times. Laser pre-heating might be an option as its also allows very fast power up and down cycles. The preheating temperature needs to be in a range where microstructure processes are slow (matter of hours see also Hernandez Paz [123]). This will allow multiple power up and down cycles without the need of the material being discarded. Induction heating frequency should be chosen based on the feedstock diameter and maximum temperature. For induction pre-heating of a $2 \mathrm{~mm}$ filament to $300^{\circ} \mathrm{C}$ the frequency should be around $75 \mathrm{kHz}$ for a skin depth of $1 / 4$ of the filament diameter. When using a threaded filament or bolt design the heating channel may need an additional constriction to remove the gas within the thread at semi-solid temperatures. All nozzle inside walls need to be coated with an applicable layer of material such as Boron-Nitride that reduces the adhesive characteristic of aluminum alloy in the semi-solid state. Chemical Vapor Deposition (CVD) of boron nitride has been effectively used for extrusion dies. This might be the preferred process of ensuring non-adhesive coating layer. Besides the induction heating before to the resistive through heating a single turn induction heating coil at the nozzle is suggested. This will reheat the prior deposited alloy layer for bonding purposes as well as keep the semi-solid temperature for currently extruded alloy material. As an alternative a selective laser heating might be used. An additional design element could be the agitation of the semi-solid slurry to decrease the 
required extrusion force (see [124]). Options for this agitation would be through a sonotrode or Magneto-Hydro-Dynamic (MHD) stirring. The nozzle components that are in contact with the semi-solid slurry should be designed to be resistive against sodium hydroxide solution.

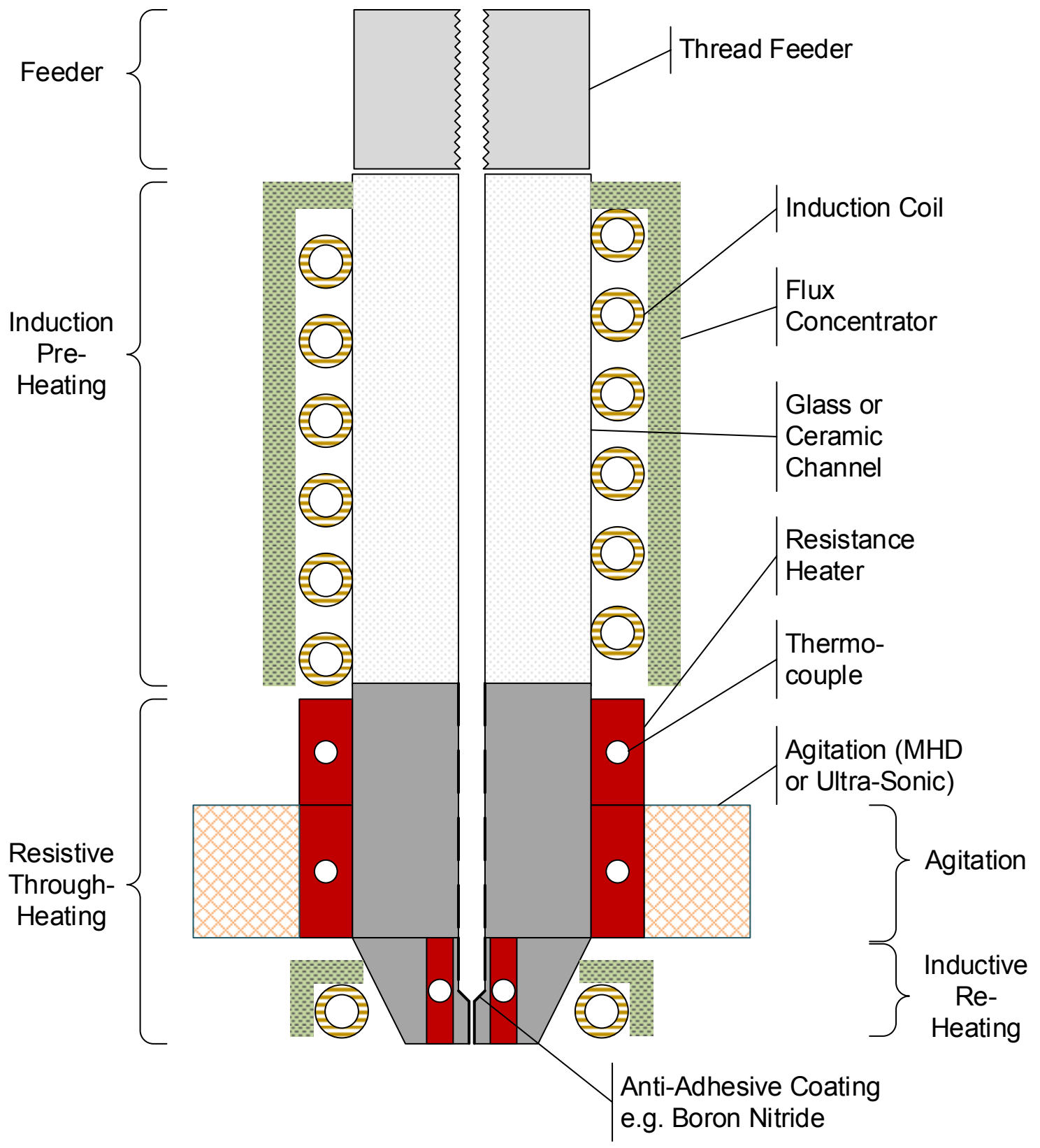

Figure 142 - Schematic MEAM nozzle design 


\section{Feeding}

In case the feed-stock will be introduced into the MEAM apparatus oxide layer free, already the storage compartment needs to be under protective gas e.g. inert gas. The force and speed process window that allows extrusion without solid liquid segregation needs to be met. For this it is assumed that a pinch roll feeder is not adequate. Instead a screw feeder used by Go et.al [70] is proposed for the MEAM apparatus to allow the application of larger forces.

For the suggested bolt material feedstock, a magazine type storage is proposed. Each bolt will be moved from a horizontal position into a vertical to be connected with the prior bolt and then pulled into the feeder as well as thereafter the heating nozzle. This allows processing of the potentially larger diameter bolt feedstock without bending. The individual bolt can be moved through the curve by the weight of the next bolts or a conveyer belt. 


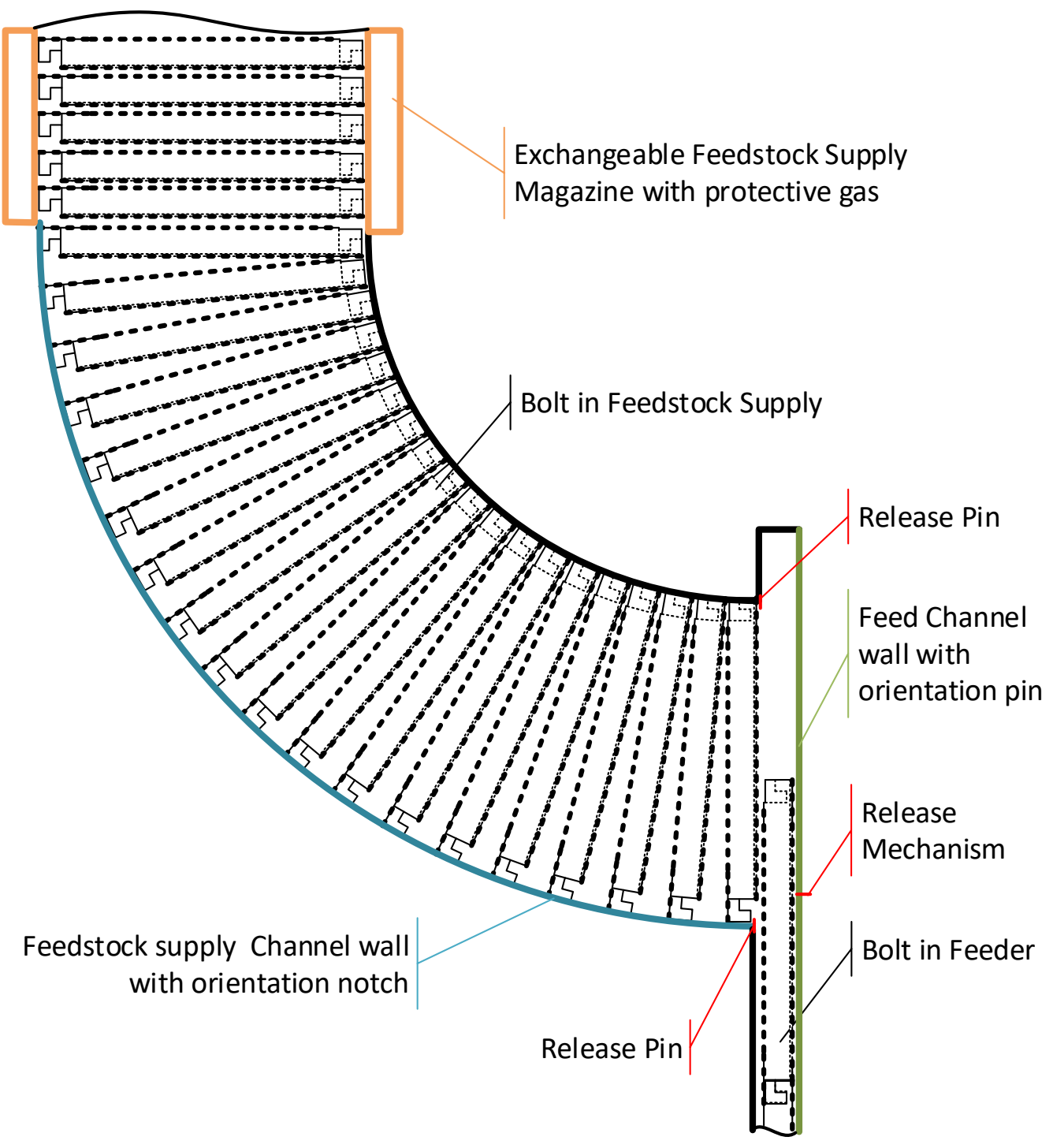

Figure 143 - Schematic feedstock supply

\section{MEAM apparatus / housing}

Within the housing or build area of the MEAM apparatus the temperature should not be elevated as the layer deposited needs to be cooled as fast as possible for better material properties of the printed product. As such a selective heating approach is necessary to ensure the prior layer is reheated for bonding purposes. While the layer deposited is cooling, oxidation needs to be prevented to allow a potential next layer to bond without an intermediate oxide layer. For this it is required to ensure inert gas atmosphere within the housing of the MEAM apparatus. Due to the complexity of the nozzle, feeder, feed stock storage and 
selective re-heating it is proposed that the build table performs all required movements in the $x-y-z$ axes. Within the MEAM apparatus a waste area is needed to extrude material from the nozzle that has reached its maximum age. A sacrifice plate or quenching waste bin is suggested which will be connected to the build table.

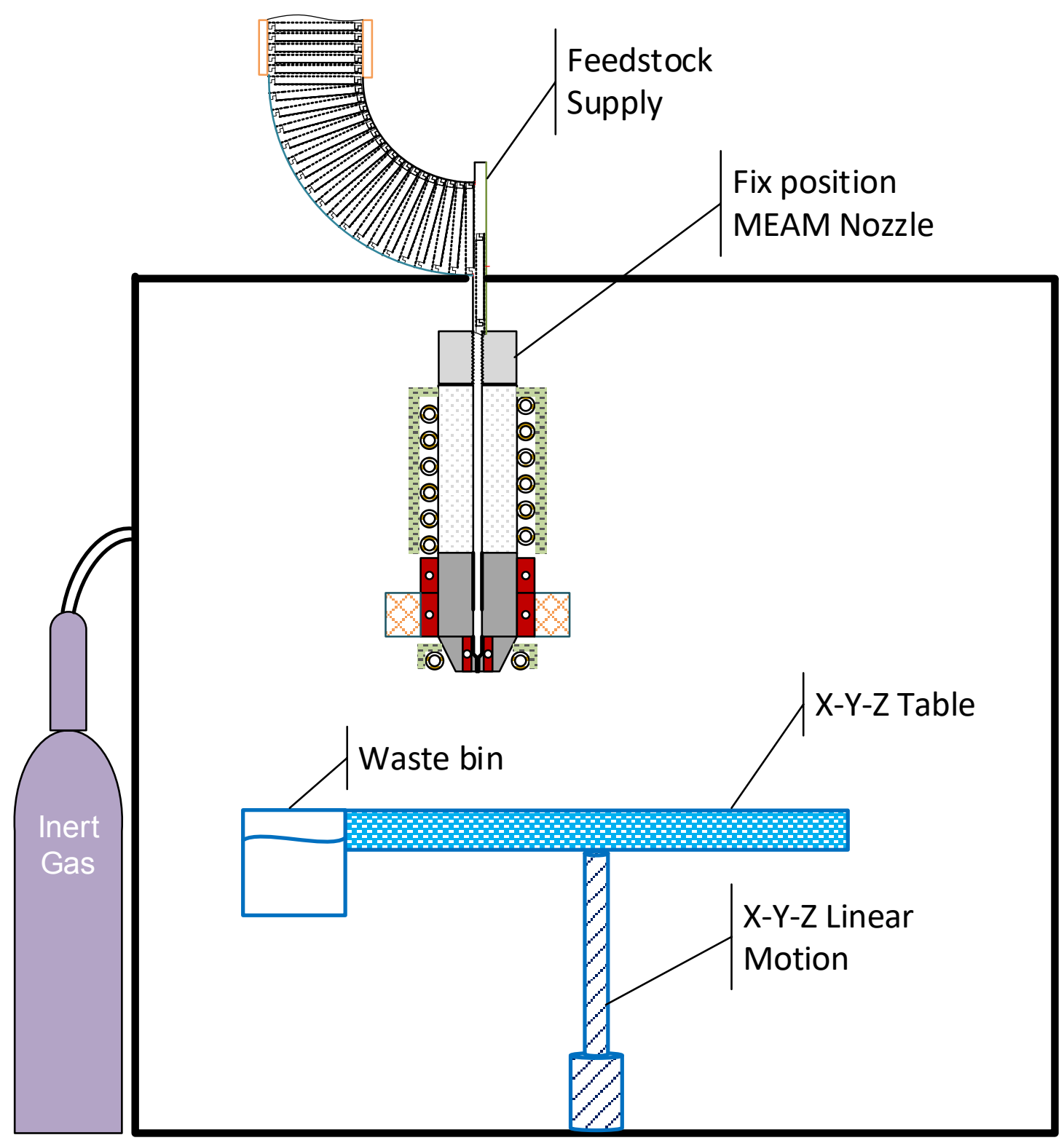

Figure 144 - MEAM apparatus - including table and housing 


\subsection{MEAM Process parameter}

The MEAM extrusion requires a precise temperature control $\left( \pm 2^{\circ} \mathrm{C}\right)$ for the extrusion at the semi-solid target temperature to achieve a $50 \%-60 \%$ fraction liquid. Especially in the complex case of an induction pre-heating, through heating resistive circuit, nozzle tip resistive heating circuit and induction reheating outside the nozzle tip controlling the interaction between all heating components needs to be designed. Critical conditions are start / stop and change of velocity.

The feeding control system needs to allow the acceleration for the startup to be high enough to reach the velocity / force process window as soon as possible. The control system must also be able to detect the force / velocity situations that would indicate clogging at the nozzle tip. This is important as in worst case a continuous feeding may result in back extrusion of semi-solid alloy into the nozzle which would damage the upper area such as the induction pre-heating components.

The control system of the MEAM apparatus needs to identify when holding time at semi solid temperature is at the maximum time for aging. This might need to be adjusted for different orifice, grain size and aging (coarsening) speed of the used alloy. Models as defined in 5.5.2 need to be loaded for each alloy type used. In case of bolt feedstock delivered in magazines the grain size and alloy type might be coded into the magazine itself so that the adjustment of the control system is automated and operator errors are minimized. As soon as the holding time is reached an extrusion process into the waste area is executed to prevent clogging.

For a mass production apparatus in-build cleaning needs to be reviewed to decrease the maintenance processes. 


\section{CONCLUSION}

This work has modeled and validated operational conditions for extrusion of aluminum alloy in a semi-solid state. Critical aspects that have been found are the composition and microstructure status of the alloy (feedstock), significance of the temperature control throughout the heating and extrusion process, the holding time at the elevated temperature as well as the velocity / force process window.

Existing filament feedstock are not ready to be processed in a semi solid state. Also, extrusion molding instead of wire drawing of filament feedstock will not create process ready material. For that a new process of semi-solid casting of such feedstock needs to be developed. In this work a non-filament but bolt feedstock was proposed that is assumed to combine the production and extrusion process requirements.

While the continuous extrusion could not be reached and significant segregation has been observed, based on prior work from Finke [71] a successful extrusion with enhanced feedstock and nozzle as well as apparatus is expected.

Practical aspects for a future MEAM process such as resistive heating, induction heating and cleaning have been reviewed and confirmed as feasible.

Overall while significant design elements are to constructed and further investigation on alloy material as well as processes are to be performed the MEAM process looks promising to create a new type of 3D printing. 


\section{FUTURE WORK}

As the presented work was to model and validated the basic extrusion potential and parameters, many aspects of a practical MEAM process are still to be investigated. The list below shows the aspects that have been derived from the results of this dissertation:

- Finalize and validate the nozzle design with a combination of induction preheating and resistive through heating. Identify the number of applicable heating circuits $(\geq 2)$ for temperature variation no greater than $\pm 2^{\circ} \mathrm{C}$. Note: For initial experiments to identify the velocity/force process window and liquid fraction a longer resistive nozzle would be sufficient but may need more heating circuits to ensure the temperature window in a longer channel area for batch processing.

- Identify the appropriate pre-heating temperature that reduces the resistive heating area while not affecting the material properties (e.g. precipitations see also Hernandez Paz [123]) even after multiple cycles.

- Review resistive heating for a threaded filament or bolt as the contact conductance is significantly reduced (see 2.3.2.2 and Figure 27)

- Identify a production process for a semi-solid casted feedstock either for filament or the discussed bolt design

- Verify the feeding mechanism (pinch roll and screw feeder) to ensure the velocity and force window parameters are met.

- Verify the acceleration process to achieve the velocity / force process window that prevents segregation. Identify other supporting parameters e.g. short-term overheating to start a continuous extrusion. Material extruded during the start process should be analyzed to understand the adherence to the later extruded material properties. In case of lower material properties, a process needs to be applied to ensure such material is outside the printed product.

- Review different liquid solid fractions (between 50\%-60\%) to find the target composition for extrusion. 
- Identify the behavior of a semi-solid slurry when applying ultra-sonic agitation in terms of segregation and processing within the nozzle neck.

- Review the production and processing of bolt designs to verify and minimize porosity

- Review deposited layer reheating approaches, e.g. induction heating based. Verify flux concentrators for targeted heating or laser-based reheating

- Review cleaning processes to verify the Boron-Nitride coating and other components (such as induction coils and flux concentrators) show resistance against sodium hydroxide solution.

- Review process parameters and design decision for higher speed MEAM. 


\section{REFERENCES}

1. Spencer, D.B., Ph.D. Thesis 1971, Massachusetts Institute of Technology: Cambridge, MA.

2. Steffi Finke, W.W., Frits Feenstra, Extrusion and Deposition of semi-solid metals, R.P.R.D.D. TNO Institute of Industrial Technology, The Netherlands / University of Twente - W.E.I. Consulting, Enschede, The Netherlands Editor. p. 639.

3. Rice, C.S., P.F. Mendez, and S.B. Brown, Metal solid freeform fabrication using semi-solid slurries. JOM, 2000. 52(12): p. 31-33.

4. Vijh, G., et al., Solid freeform fabrication of aluminum alloy components: Numerical simulations. Transactions of the Indian Institute of Metals, 2009. 62(4-5): p. 291-4.

5. Miyamoto, Y. and C. Wenbin, Freeform fabrication of aluminum parts by direct deposition of molten aluminum. Journal of Materials Processing Technology, 2006. 173(2): p. 209-12.

6. Qi, L.-h., et al., A novel selection method of scanning step for fabricating metal components based on micro-droplet deposition manufacture. International Journal of Machine Tools and Manufacture, 2012. 56: p. 50-58.

7. Callister, W.D. and D.G. Rethwisch, Materials Science and Engineering: An Introduction, Eigth Edition Binder Ready Version. 2010: John Wiley \& Sons.

8. Bargel, H.J. and G. Schulze, Werkstoffkunde. 2012: Springer.

9. Hirth, J.P. and J. Lothe, Theory of dislocations. 1982: Krieger Pub. Co.

10. http://www.aluminium-brazing.com/tag/metallurgy/. [cited 201220 Sep].

11. Barnes, H.A., Thixotropy a review. Journal of Non-Newtonian Fluid Mech., 70 (1997) 1-33, 1997. 70: p. 34.

12. Flemings, M., Behavior of metal alloys in the semisolid state. Metallurgical Transactions B (Process Metallurgy), 1991. 22B(3): p. 269-93.

13. Assistance, M.E.O.o.E.A.O.o.T., Semi-solid Metal Casting: Reducing the Cost of Copper Alloy Parts. 2003: Massachusetts Office of Technical Assistance.

14. Scheil, E., Bemerkungen zur Schichtkristallbildung. Zeitschrift für Metallkunde, 1942. 34: p. 70-72.

15. Kirkwood, D.H., Semi-solid processing of alloys. 2009, New York: Springer. 
16. Loué, W.R. and M. Suéry, Microstructural evolution during partial remelting of $\mathrm{Al} \cdot$ Si7Mg alloys. Materials Science and Engineering: A, 1995. 203(1-2): p. 1-13.

17. (ASTM), A.S.f.T.a.M., Volume 03.01 Metals -- Mechanical Testing; Elevated and Low-Temperature Tests; Metallography. 2012. p. 1636.

18. Tzimas, E. and A. Zavaliangos, Evaluation of volume fraction of solid in alloys formed by semisolid processing. Journal of Materials Science, 2000. 35(21): p. 5319-5329.

19. Gurland, J., The Measurement of Grain Contiguity in Two-Phase alloys, in Trans. AIME, 212. 1958. p. 452-455.

20. Ostwald, W., Ueber die rechnerische Darstellung des Strukturgebietes der Viskosität. Kolloid-Zeitschrift, 1929. 47(2): p. 176-187.

21. Herschel, W. and R. Bulkley, Konsistenzmessungen von GummiBenzollösungen. Kolloid-Zeitschrift, 1926. 39(4): p. 291-300.

22. Kumar, P., M.I.o.T.D.o.M. Science, and Engineering, Constitutive Modeling and Characterization of the Flow Behavior of Semi-solid Metal Alloy Slurries. 1994: Massachusetts Institute of Technology, Department of Materials Science and Engineering.

23. Pan, Q.Y., D. Apelian, and A.N. Alexandrou, Yield behavior of commercial Al-Si alloys in the semisolid state. Metallurgical and Materials Transactions B: Process Metallurgy and Materials Processing Science, 2004. 35(6): p. 1187-1202.

24. Sigworth, G.K., Rheological properties of metal alloys in the semi-solid state. Canadian Metallurgical Quarterly, 1996. 35(2): p. 101-122.

25. Casting Simulation Suite. [cited 2013 25-July]; Available from: http://www.esi-group.com/products/casting/casting-simulation-suite.

26. Orgeas, L., et al., Modelling of semi-solid processing using a modified temperature-dependent power-law model. Modelling and Simulation in Materials Science and Engineering, 2003. 11(4): p. 553-74.

27. Gebelin, J.C.F., D.; Suery, M., Comparison of One and Two-Phase Approaches for Numerical Simulation of Semi-Solid Processing, in 5th International conference Semi-solid processing of alloys and composites. 1998, Colorado School of Mines, Golden, CO. p. 309-316.

28. Nguyen, T.G., D. Favier, and M. Suery, Theoretical and experimental study of the isothermal mechanical behaviour of alloys in the semi-solid state. International Journal of Plasticity, 1994. 10(6): p. 663-93.

29. Martin, C.L., D. Favier, and M. Suery, Viscoplastic behaviour of porous metallic materials saturated with liquid. I. Constitutive equations. International Journal of Plasticity, 1997. 13(3): p. 215-35.

30. Martin, C.L., D. Favier, and M. Suery, Viscoplastic behaviour of porous metallic materials saturated with liquid. II. Experimental identification on 
a Sn-Pb model alloy. International Journal of Plasticity, 1997. 13(3): p. 237-59.

31. Pineau, F. Reactive two-phase flow model for materials in the semi-solid state. in Eighth International Conference on Advanced Computational Methods in Heat Transfer, HEAT TRANSFER VIII, March 24, 2004 - March 25, 2004. 2004. Lisbon, Portugal: WITPress.

32. Atkinson, H.V., Modelling the semisolid processing of metallic alloys. Progress in Materials Science, 2005. 50(3): p. 341-412.

33. Uggowitzer, P.J., et al., Metallkundliche Aspekte bei der semi-solid Formgebung von Leichtmetallen. 2000: Institut für Metallforschung, ETH Zürich.

34. ANSI H35.1 American national standard alloy and temper designation systems for aluminum (U.S. \& Metric Units). 2004: Aluminum Association.

35. AA 354, C355 \& A356 Foundry Alloys, in Product Bulletin, A.P.M. Group, Editor. 2007.

36. H. Kaufmann, W.F., U. Galovsky, P. J. Uggowitzer, Einfluss von Schwankungen der Zusammensetzung der Legierung AlSi7Mg auf das Fließvermögen im flüssigen und im Semi-solid Zustand. DRUCKGUSSPRAXIS, 2005. 5: p. 9.

37. Zoqui, E.J., M. Paes, and E. Es-Sadiqi, Macro- and microstructure analysis of SSM A356 produced by electromagnetic stirring. Journal of Materials Processing Technology, 2002. 120(1-3): p. 365-373.

38. Drouzy, M., S. Jacob, and M. Richard, INTERPRETATION OF TENSILE RESULTS BY MEANS OF QUALITY INDEX AND PROBABLE YIELD STRENGTH. International cast metals journal, 1980. 5(2): p. 43-50.

39. F. Khomamizadeh, A.G., Evaluation of quality index A356 aluminium alloy by microstructural analysis in Scientia Iranica, S.U.o. Technology, Editor. 2004. p. 386 - 391.

40. Alexopoulos, N.D., Definition of quality in cast aluminum alloys its characterization with appropriate indices. Journal of Materials Engineering and Performance, 2006. 15(1): p. 59-66.

41. James A. Yurko, R.A.M., Merton C. Fleming, Commercial Development of the Semi-Solid Rheocasting (SSR ${ }^{\text {TM }}$ ) Process in North American Die Casting Association (NADCA) 22nd International Congress. 2003.

42. Thanabumrungkul, S., et al., Industrial development of gas induced semisolid process. Transactions of Nonferrous Metals Society of China, 2010. 20, Supplement 3(0): p. s1016-s1021.

43. Hirt, G. and R. Kopp, Thixoforming. 2009: John Wiley \& Sons.

44. K.P. Young, E.D., P. Harvey, W. Gary, Process and Apparatus for Continuous Slurry Casting 1984: USA.

45. Kenneth P. Young, C.P.K., James A. Courtois, Fine Grained Metal Composites, U.S. Patent, Editor. 1983: USA. p. 9. 
46. Tzimas, E., Evolution of Microstructure and Rheological Behavior of Alloys in the Semisolid State. 1997: Drexel University.

47. Jr., K.T.H., Equal Channel Angular Extrusion Method, U.S. Patent, Editor. 2005: USA.

48. Terhune, S., et al., An investigation of microstructure and grain-boundary evolution during ECA pressing of pure aluminum. Metallurgical and Materials Transactions A, 2002. 33(7): p. 2173-2184.

49. Jiang, J.-f. and S.-j. Lou, Preparation of semi-solid billet of magnesium alloy and its thixoforming. Transactions of the Nonferrous Metals Society of China, 2007. 17(1): p. 46-50.

50. Ashouri, S., et al., Semi-solid microstructure evolution during reheating of aluminum A356 alloy deformed severely by ECAP. Journal of Alloys and Compounds, 2008. 466(1-2): p. 67-72.

51. P. Groche, C.M., M. Görtan, E. Bruder, Kontinuierliche Herstellung hochfester ultra-feinkörniger Drähte mithilfe des ECAS-Prozesses. Werkstattstechnik online, 2010(10): p. 8.

52. Sauermann, R., Design von Aluminium-Lithium-Legierungen für das Thixoforming. 2006: Shaker.

53. Noll, T., Die anwendungsgerechte Weiterentwicklung des Aluminiumwerkstoffes EN AC-AISi7Mg0,3(A356) für das Thixoforming mit chemischer Kornfeinung. 2003: Shaker.

54. ASTM, Standard Terminology for Additive Manufacturing Technologies. 2012.

55. Gibson, I., D.W. Rosen, and B. Stucker, Additive Manufacturing Technologies: Rapid Prototyping to Direct Digital Manufacturing. 2009: Springer.

56. Homepage of Align Technology. [cited 2012 04th Nov]; Available from: http://www.aligntech.com.

57. Adriaan Bernardus Spierings, T.L.S., Konrad Wegener, Fatigue performance of Additive Manufactured metallic Parts. Rapid Prototyping Journal,, 2012. 19(2).

58. Anderson, C., Makers: the new industrial revolution. 2012: Random House Business Books.

59. Wohlers, T., Developments in Additive Manufacturing. Manufacturing Engineering, 2010. 144(1): p. 54-55,57-59.

60. Anderson, C. and K.A. Gilles, Advances in wire-feeder technology. Welding Design and Fabrication, 2006. 79(3): p. 24-6.

61. Garzón-Alvarado, D.A., M.A. Velasco, and C.A. Narváez-Tovar, Modeling porous scaffold microstructure by a reaction-diffusion system and its degradation by hydrolysis. Computers in Biology and Medicine, 2012. 42(2): p. 147-155.

62. User Guide uPrint. 2008, Stratasys Inc. 
63. GmbH, S. Fabbster Home Page. [cited 2013 21th April]; Available from: www.fabbster.com.

64. THE FABULOUS, FANTASTIC AND FUNCTIONAL FABBSTER. [cited 2013 21th April]; Available from:

http://solidsmack.com/fabrication/fabulous-fabbster-3d-printer/.

65. Andreas Hess, M.A., Welding wire feeding device comprising at least two rollers driven by a worm gear, E.P. Register, Editor. 2005: EU.

66. Andreas Hess, M.A., Apparatus for feeding wire for welding device with device for measuring the wire speed, E.P. Register, Editor. 2003: EU.

67. Salzmann, A. Schweissaufsichtspersonal - Fachordner der SIA 161 Norm Schweissbewilligung S1 und S2. 2003 [cited 2013 08-Jun]; Available from: http://schweissaufsicht.ansa.ch/.

68. Berger;, T.P.T.P.Q.D.R.M.R.A.R.J.R. Dynamic Factors Contributing to Buckling and Birdnesting During GMAW Wire Feeding. in AWS Intl. Exposition and Annual Convention. 2005. Dallas, TX.

69. Go, J., et al., Rate limits of additive manufacturing by fused filament fabrication and guidelines for high-throughput system design. Additive Manufacturing, 2017. 16: p. 1-11.

70. Go, J. and A.J. Hart, Fast Desktop-Scale Extrusion Additive Manufacturing. Additive Manufacturing, 2017. 18: p. 276-284.

71. Finke, S., Solid freeform fabrication of metal components by extrusion and deposition of semi-solid metals. 2002, University of Twente: Enschede. p. 131.

72. Yuu, O., et al., Experimental investigations on monitoring and control of induction heating process for semi-solid alloys using the heating coil as sensor. Measurement Science \&amp; Technology, 2002. 13(8): p. 135965.

73. Incropera, F.P., Fundamentals of heat and mass transfer. 2007: John Wiley.

74. Ritt, H.-M., Regelung der induktiven Erwärmung von Metallen in den teilflüssigen Zustand. 2000, VDI-Verl.: Düsseldorf.

75. Systems, D. SolidWorks Help - Thermal Contact Resitance. [cited 2013 7th July]; Help for SolidWorks Simulation > Meshing with Contact > Thermal Contact Resistance]. Available from: http://help.solidworks.com/2012/English/SolidWorks/cworks/Ther mal Contact Resistance.htm.

76. Doege, E. and B.A. Behrens, Handbuch Umformtechnik: Grundlagen, Technologien, Maschinen. 2010: Springer London, Limited.

77. SB. Induction Heating. 2009 [cited 2013 18th July]; RepRap Builders Blog entry for induction heating of a RepRap Extruder]. Available from: http://builders.reprap.org/2009/05/induction-heating.html.

78. Pfeiffer, H., Taschenbuch Industrielle Wärmetechnik. 2007: VulkanVerlag. 
79. Davies, J., Conduction and Induction Heating. 1990: P. Peregrinus Limited.

80. Conrad, H., A. Mühlbauer, and R. Thomas, Elektrothermische Verfahrenstechnik. 1994: Vulkan-Verlag Classen.

81. B.Avitzur, C.N., Y.T.Chou, Upper-Bound Solution to Flow Through Conical Converging dies. 1980, Information Bridge: DOE Scientific and Technical Information. p. 45.

82. Gordon, W.A., C.J. Van Tyne, and Y.H. Moon, Minimizing distortion during extrusion using adaptable dies. International Journal of Mechanical Sciences, 2012.62(1): p. 1-17.

83. Kalpakjian, S. and S.R. Schmid, Manufacturing Engineering and Technology. 2006: Perarson/Prentice Hall.

84. Lerner, L.S., Physics: for scientists and engineers ; Modern physics : for scientists and engineers. 1996: Jones and Bartlett.

85. Kalpakjian, S. and S.R. Schmid, Manufacturing processes for engineering materials. 2010: Pearson education.

86. Michaeli, W., Extrusion Dies for Plastics and Rubber: Design and Engineering Computations. 2003: Hanser Gardner Publications.

87. C.J. Paradies, M.R., T. Imwinkelried, J.P. Gabathuler, Simulation of the Pressure Die Casting Process of a Thixotropic Aluminium Alloy, in 4th International Conference on Semi-Solid Processing of Al- loys and Composites. 1996, Department of Engineering Materials, University of Sheffield: Sheffield, UK. p. 115.

88. Blazynski, T.Z., Applied elasto-plasticity of solids. 1983: Macmillan Press.

89. Miles, N., G. Evans, and A. Middleditch, Bearing lengths for extrusion dies: Rationale, current practice and requirements for automation. Journal of Materials Processing Technology, 1997. 72(1): p. 162-176.

90. Kang, C.G., H.K. Jung, and K.W. Jung, Thixoforming of an aluminum component with a die designed by process simulation. Journal of Materials Processing Technology, 2001. 111(1-3): p. 37-41.

91. Yardimci, M.A. and S. Guceri, Conceptual framework for the thermal process modelling of fused deposition. Rapid Prototyping Journal, 1996. 2(2): p. 26-31.

92. Costa, S.F., F.M. Duarte, and J.A. Covas, Towards modelling of Free Form Extrusion: Analytical solution of transient heat transfer. International Journal of Material Forming, 2008. 1(SUPPL. 1): p. 703-706.

93. Carslaw, H.S. and J.J.C. Jaeger, CONDUCTION OF HEAT IN SOLIDS. 2nde edition, Edition anglaise. 1986: Clarendo Press.

94. Sun, Q., et al., Effect of processing conditions on the bonding quality of FDM polymer filaments. Rapid Prototyping Journal, 2008. 14(2): p. 72-80.

95. Swanson, W.J.T., Patrick W.; Leavitt, Paul J.; Karwoski, Peter J.; LaBossiere Joseph E.; Skubic, Robert L., High temperature modeling apparatus 2001, Stratsys Inc. . p. 4. 
96. Partain, S.C., Fused deposition modeling with localized pre-deposition heating using forced air in Department of Mechanical and Industrial Engineering. 2007, MONTANA STATE UNIVERSITY Bozeman, Montana

97. Park, K. and S.-I. Lee, Localized mold heating with the aid of selective induction for injection molding of high aspect ratio micro-features. Journal of Micromechanics and Microengineering, 2010. 20(3).

98. Sergeant, P., et al., Thermal analysis of magnetic shields for induction heating. IET Electric Power Applications, 2009. 3(6): p. 543-50.

99. Wu, G., et al., Solid freeform fabrication of metal components using fused deposition of metals. Materials and Design, 2002. 23(1): p. 97-105.

100. M.K. Agarwala, R.v.W., A. Bandyopadhyay, P.J. Whalen, A. Safari, S.C. Danforth, Fused Deposition of Ceramics and Metals: An Overview. 1996: DARPA, ONR (Contract-No. 00014-94-0115).

101. Mostafa, N., et al., A Study of Melt Flow Analysis of an ABS-Iron Composite in Fused Deposition Modelling Process. Tsinghua Science and Technology, 2009. 14(SUPPL. 1): p. 29-37.

102. Masood, S.H. and W.Q. Song, Thermal characteristics of a new metal/polymer material for FDM rapid prototyping process. Assembly Automation, 2005. 25(4): p. 309-15.

103. Mendez, P.F., Joining metals using semi-solid slurries. 1995, Massachusetts Institute of Technology. p. 27 leaves.

104. Rice, C.S., Solid freeform fabrication using semi-solid processing. 1995, Massachusetts Institute of Technology. p. 48 leaves.

105. Brown, S.B., Method and apparatus for metal solid freeform fabrication utilizing partially solidified metal slurry, U.S.P.a.T. Office, Editor. 1997, Stuart B. Brown: USA.

106. Stuart B. Brown, P.F.M., Method and apparatus for metal solid freeform fabrication utilizing partially solidified metal slurry, U.S.P.a.T. Office, Editor. 1999, Semi Solid Technologies Inc.: USA.

107. Secretary of the Commonwealth, C.D., SEMI-SOLID TECHNOLOGIES, INC. Summary Screen 2013.

108. Finke, S. and F.K. Feenstra, Solid freeform fabrication by extrusion and deposition of semi-solid alloys. Journal of Materials Science, 2002. 37(15): p. 3101-6.

109. Crump, S.S., Apparatus and method for creating three-dimensional objects, U.S.P.a.T. Office, Editor. 1992, Stratasys, Inc.: USA.

110. William J. Swanson, P.W.T., Paul J. Leavitt, Peter J. Karwoski, Joseph E. LaBossiere, Robert L. Skubic, High temperature modeling apparatus, U.S.P.a.T. Office, Editor. 2004, Stratasys, Inc.: USA.

111. Scott S. Crump, J.S.B., Timothy Sampson, Robert L. Zinniel, John Barnett, System and Method for Building Three-Dimensional Objects with metalbased Alloys, U.S.P.a.T. Office, Editor. 2011, Stratosys, Inc.: USA. 
112. Mireles, J., et al., Development of a fused deposition modeling system for low melting temperature metal alloys. Journal of Electronic Packaging, Transactions of the ASME, 2013. 135(1).

113. Chen, W., et al., Direct metal writing: Controlling the rheology through microstructure. Vol. 110. 2017. 094104.

114. Garcia, N.M., Fusion of aluminum AA6061-T6 using induction heating for the purpose of additive manufacturing. Fusion of aluminum AA6061-T6 using induction heating for the purpose of additive manufacturing, 2016.

115. Mireles, J., et al., Fused Deposition Modeling of Metals.

116. Drahtwerke Elisental. [cited 2013 8th Aug]; Available from: http://www.elisental.de/.

117. Foundry Institute - RHTW Aachen [cited 2013 1st Aug]; Available from: www.gi.rwth-aachen.de/.

118. Elmotec Antriebstechnik AG. 2013 [cited 2013 1st Aug]; Solder Wire Feeder ]. Available from: http://www.elmotec.ch/elmotecen/loettechnik/loetdrahtvorschub.php.

119. Rep-Strap Wiki page within the Rep-Rap community. [cited 2013 1st Aug]; Available from: http://reprap.org/wiki/RepStrap.

120. Haimbaugh, R.E., Practical Induction Heat Treating. 2001: ASM International.

121. Julious, S.A., Sample sizes for clinical trials. 2010, Boca Raton :: CRC Press/Taylor \& Francis.

122. Moradi, M., et al., Investigation of Thin Wall Casting Made of Semi-Solid A356 Using Back Extrusion and Die Cast. Solid State Phenomena, 2008. 141-143: p. 67-72.

123. Paz, J.F.H., Heat Treatment and Precipitation in A356 Aluminum Alloy, in Department of Mining, Metals and Materials Engineering. 2003, McGill University: Montreal, Canada.

124. Jones, J.B., et al., Ultrasonic Applied to Aluminum Extrusion Cladding of Tubes. 1959: aeroprojects Incorporated west chester pa. 


\section{APPENDIX}

\section{Heating channel measurements}

Pre-experiment:

Response: Temperature in Nozzle channel in ${ }^{\circ} \mathrm{C}$

\section{Factors:}

- Temperature sensor channel position / depth

- Temperature setting of nozzle

\section{Factor Level:}

- Temperature setting for Nozzle:
- $585^{\circ} \mathrm{C}$
- $600^{\circ} \mathrm{C}$

- Temperature sensor Depth
- $46 \mathrm{~mm}$
○ $41 \mathrm{~mm}$
○ $36 \mathrm{~mm}$
- $31 \mathrm{~mm}$
- $26 \mathrm{~mm}$
- $21 \mathrm{~mm}$
- $16 \mathrm{~mm}$
○ $11 \mathrm{~mm}$

\section{Pre-experiment:}




\begin{tabular}{|c|c|c|}
\hline Temperature setting Nozzle $\left({ }^{\circ} \mathrm{C}\right)$ & Sensor Depth in $\mathrm{mm}$ & Temp in Nozzle in ${ }^{\circ} \mathrm{C}$ \\
\hline 585 & 46 & 593 \\
\hline 585 & 41 & 590.6 \\
\hline 585 & 36 & 587 \\
\hline 585 & 31 & 578.5 \\
\hline 585 & 26 & 565.1 \\
\hline 585 & 21 & 553.2 \\
\hline 585 & 16 & 533.3 \\
\hline 585 & 11 & 493.1 \\
\hline 600 & 46 & 609.2 \\
\hline 600 & 41 & 603.9 \\
\hline 600 & 36 & 601.3 \\
\hline 600 & 31 & 591 \\
\hline 600 & 26 & 579.9 \\
\hline 600 & 21 & 566.4 \\
\hline 600 & 16 & 551.7 \\
\hline 600 & 11 & 519.6 \\
\hline 585 & 46 & 591 \\
\hline 585 & 41 & 589.5 \\
\hline 585 & 36 & 586.2 \\
\hline 585 & 31 & 577.2 \\
\hline 585 & 26 & 566.2 \\
\hline 585 & 21 & 552.2 \\
\hline 585 & 16 & 536.7 \\
\hline 585 & 11 & 496.2 \\
\hline 600 & 46 & 605 \\
\hline 600 & 41 & 602.6 \\
\hline 600 & 36 & 600.9 \\
\hline 600 & 31 & 592 \\
\hline 600 & 26 & 579.4 \\
\hline 600 & 21 & 565.4 \\
\hline 600 & 16 & 553.7 \\
\hline 600 & 11 & 520.1 \\
\hline
\end{tabular}

\section{General Linear Model:}

Factor Information

Factor Type Levels Values




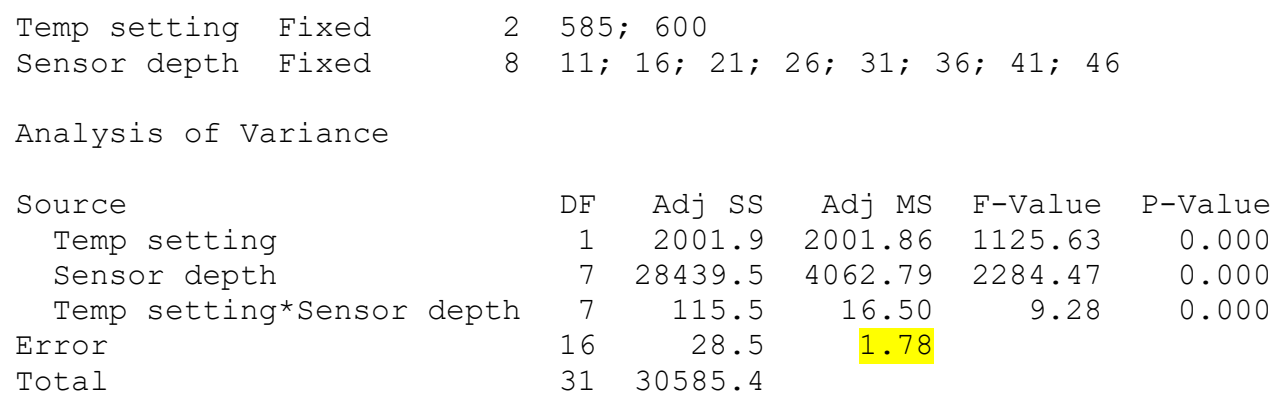

Model Summary
1.33358
$\mathrm{R}-\mathrm{sq}$
$R-s q(a d j)$
$\mathrm{R}-\mathrm{sq}$ (pred)
$99.91 \%$
$99.82 \%$
$99.63 \%$

\section{Calculation of Standard Deviation:}

MSE $=1.78 \sigma=\sqrt{M S_{E}}=1.3342$

Power and sample size:

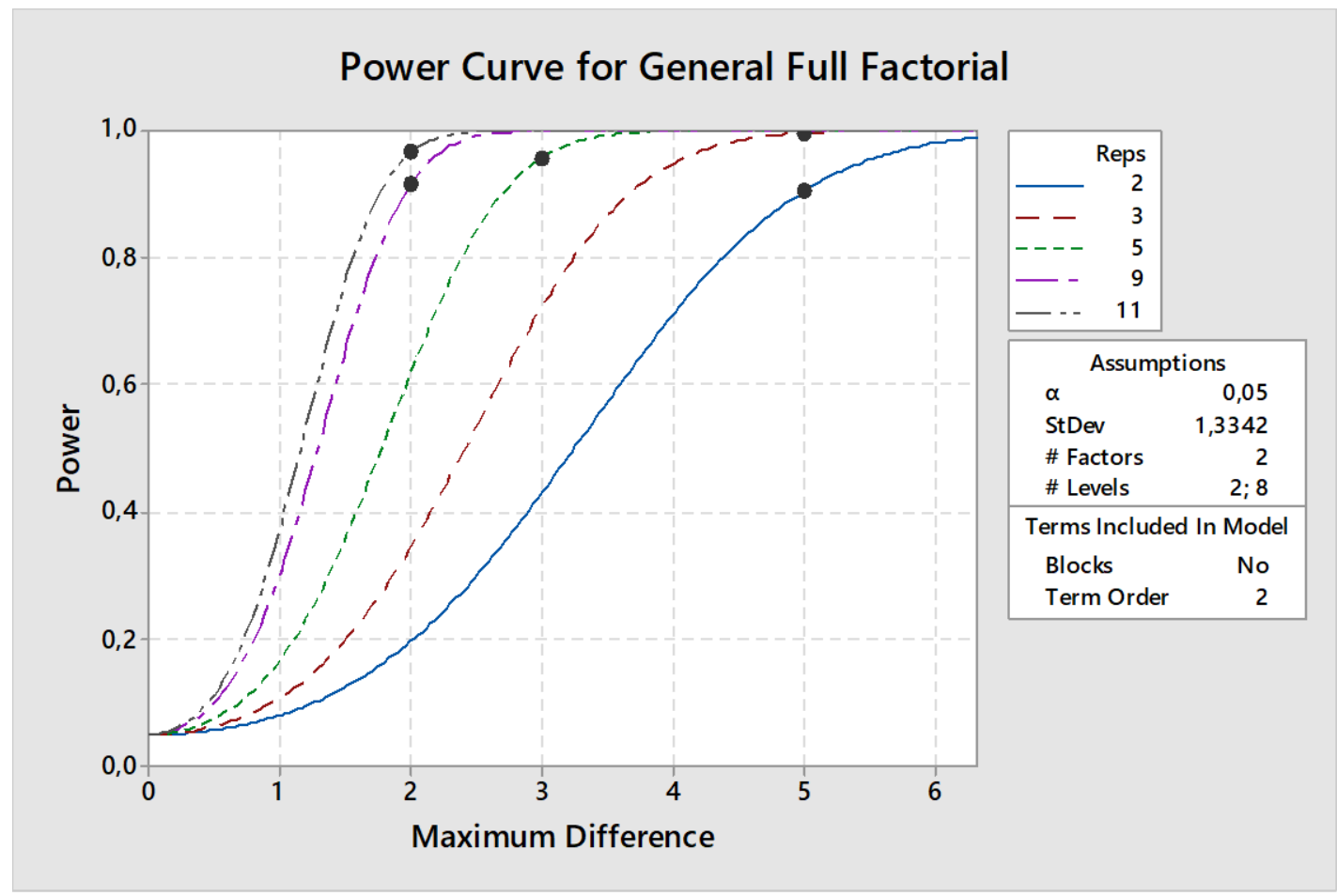

\footnotetext{
General Full Factorial Design

$\alpha=0.05$ Assumed standard deviation $=1.3342$

Factors: 2 Number of levels: 2; 8

Include terms in the model up through order: 2

Not including blocks in model.
} 


$\begin{array}{rrrrr}\text { Maximum } & & \text { Total } & \text { Target } & \\ \text { Difference } & \text { Reps } & \text { Runs } & \text { Power } & \text { Actual Power } \\ 2 & 9 & 144 & 0.90 & 0.915110 \\ 2 & 11 & 176 & 0.95 & 0.965694 \\ 3 & 5 & 80 & 0.90 & 0.957930 \\ 3 & 5 & 80 & 0.95 & 0.957930 \\ 5 & 2 & 32 & 0.90 & 0.905255 \\ 5 & 3 & 48 & 0.95 & 0.996664\end{array}$

Results:

\begin{tabular}{|c|c|c|c|c|c|c|r|}
\hline $\begin{array}{c}\text { Std } \\
\text { Order }\end{array}$ & $\begin{array}{c}\text { Run } \\
\text { Order }\end{array}$ & $\begin{array}{c}\text { Temp } \\
\text { coded }\end{array}$ & $\begin{array}{c}\text { Sensor } \\
\text { Depth } \\
\text { coded }\end{array}$ & $\begin{array}{c}\text { Temp } \\
\text { Setting }\end{array}$ & $\begin{array}{c}\text { Sensor } \\
\text { Depth }\end{array}$ & $\begin{array}{c}\text { Delta } \\
\text { Temp }\end{array}$ & $\begin{array}{c}\text { Measured } \\
\text { Temp. }\end{array}$ \\
\hline 8 & 1 & 1 & 8 & 585 & 11 & -94.6 & 490.4 \\
\hline 40 & 3 & 1 & 8 & 585 & 11 & -83.9 & 501.1 \\
\hline 72 & 16 & 1 & 8 & 585 & 11 & -94.7 & 490.3 \\
\hline 16 & 29 & 2 & 8 & 600 & 11 & -79.4 & 520.6 \\
\hline 48 & 31 & 2 & 8 & 600 & 11 & -78.0 & 522.0 \\
\hline 56 & 47 & 1 & 8 & 585 & 11 & -80.1 & 504.9 \\
\hline 24 & 51 & 1 & 8 & 585 & 11 & -82.9 & 502.1 \\
\hline 80 & 71 & 2 & 8 & 600 & 11 & -111.0 & 489.0 \\
\hline 64 & 73 & 2 & 8 & 600 & 11 & -101.1 & 498.9 \\
\hline 32 & 78 & 2 & 8 & 600 & 11 & -106.7 & 493.3 \\
\hline 71 & 4 & 1 & 7 & 585 & 16 & -44.5 & 540.5 \\
\hline 39 & 17 & 1 & 7 & 585 & 16 & -39.2 & 545.8 \\
\hline 63 & 20 & 2 & 7 & 600 & 16 & -46.7 & 553.3 \\
\hline 7 & 24 & 1 & 7 & 585 & 16 & -42.7 & 542.3 \\
\hline 79 & 33 & 2 & 7 & 600 & 16 & -44.7 & 555.3 \\
\hline 15 & 37 & 2 & 7 & 600 & 16 & -44.9 & 555.1 \\
\hline 31 & 39 & 2 & 7 & 600 & 16 & -42.7 & 557.3 \\
\hline 23 & 42 & 1 & 7 & 585 & 16 & -49.0 & 536.0 \\
\hline 47 & 48 & 2 & 7 & 600 & 16 & -43.6 & 556.4 \\
\hline 55 & 50 & 1 & 7 & 585 & 16 & -50.0 & 535.0 \\
\hline 6 & 6 & 1 & 6 & 585 & 21 & -27.5 & 557.5 \\
\hline 22 & 9 & 1 & 6 & 585 & 21 & -23.9 & 561.1 \\
\hline 46 & 18 & 2 & 6 & 600 & 21 & -31.8 & 568.2 \\
\hline 78 & 28 & 2 & 6 & 600 & 21 & -25.1 & 574.9 \\
\hline 70 & 38 & 1 & 6 & 585 & 21 & -26.2 & 558.8 \\
\hline
\end{tabular}




\begin{tabular}{|c|c|c|c|c|c|c|r|}
\hline $\begin{array}{c}\text { Std } \\
\text { Order }\end{array}$ & $\begin{array}{c}\text { Run } \\
\text { Order }\end{array}$ & $\begin{array}{c}\text { Temp } \\
\text { coded }\end{array}$ & $\begin{array}{c}\text { Sensor } \\
\text { Depth } \\
\text { coded }\end{array}$ & $\begin{array}{c}\text { Temp } \\
\text { Setting }\end{array}$ & $\begin{array}{c}\text { Sensor } \\
\text { Depth }\end{array}$ & $\begin{array}{c}\text { Delta } \\
\text { Temp }\end{array}$ & $\begin{array}{c}\text { Measured } \\
\text { Temp. }\end{array}$ \\
\hline 62 & 64 & 2 & 6 & 600 & 21 & -24.9 & 575.1 \\
\hline 30 & 66 & 2 & 6 & 600 & 21 & -25.5 & 574.5 \\
\hline 54 & 69 & 1 & 6 & 585 & 21 & -21.3 & 563.7 \\
\hline 38 & 72 & 1 & 6 & 585 & 21 & -25.0 & 560.0 \\
\hline 14 & 76 & 2 & 6 & 600 & 21 & -25.4 & 574.6 \\
\hline 69 & 2 & 1 & 5 & 585 & 26 & -10.6 & 574.4 \\
\hline 53 & 7 & 1 & 5 & 585 & 26 & -11.1 & 573.9 \\
\hline 29 & 15 & 2 & 5 & 600 & 26 & -12.7 & 587.3 \\
\hline 21 & 21 & 1 & 5 & 585 & 26 & -11.2 & 573.8 \\
\hline 37 & 30 & 1 & 5 & 585 & 26 & -11.6 & 573.4 \\
\hline 45 & 46 & 2 & 5 & 600 & 26 & -20.2 & 579.8 \\
\hline 61 & 49 & 2 & 5 & 600 & 26 & -14.0 & 586.0 \\
\hline 77 & 54 & 2 & 5 & 600 & 26 & -10.9 & 589.1 \\
\hline 13 & 61 & 2 & 5 & 600 & 26 & -13.4 & 586.6 \\
\hline 5 & 80 & 1 & 5 & 585 & 26 & -10.6 & 574.4 \\
\hline 76 & 11 & 2 & 4 & 600 & 31 & 0.5 & 600.5 \\
\hline 68 & 12 & 1 & 4 & 585 & 31 & 1.3 & 586.3 \\
\hline 28 & 19 & 2 & 4 & 600 & 31 & -0.1 & 599.9 \\
\hline 20 & 23 & 1 & 4 & 585 & 31 & -2.0 & 583.0 \\
\hline 44 & 25 & 2 & 4 & 600 & 31 & -0.7 & 599.3 \\
\hline 4 & 26 & 1 & 4 & 585 & 31 & -0.6 & 584.4 \\
\hline 60 & 35 & 2 & 4 & 600 & 31 & 0.4 & 600.4 \\
\hline 36 & 41 & 1 & 4 & 585 & 31 & -0.9 & 584.1 \\
\hline 12 & 60 & 2 & 4 & 600 & 31 & 1.6 & 601.6 \\
\hline 52 & 77 & 1 & 4 & 585 & 31 & -2.0 & 583.0 \\
\hline 3 & 8 & 1 & 3 & 585 & 36 & 6.4 & 591.4 \\
\hline 43 & 14 & 2 & 3 & 600 & 36 & 8.9 & 608.9 \\
\hline 19 & 34 & 1 & 3 & 585 & 36 & 4.7 & 589.7 \\
\hline 11 & 40 & 2 & 3 & 600 & 36 & 4.9 & 604.9 \\
\hline 67 & 52 & 1 & 3 & 585 & 36 & 5.4 & 590.4 \\
\hline 35 & 59 & 1 & 3 & 585 & 36 & 5.0 & 590.0 \\
\hline 51 & 63 & 1 & 3 & 585 & 36 & 5.8 & 590.8 \\
\hline
\end{tabular}




\begin{tabular}{|c|c|c|c|c|c|c|r|}
\hline $\begin{array}{c}\text { Std } \\
\text { Order }\end{array}$ & $\begin{array}{c}\text { Run } \\
\text { Order }\end{array}$ & $\begin{array}{c}\text { Temp } \\
\text { coded }\end{array}$ & $\begin{array}{c}\text { Sensor } \\
\text { Depth } \\
\text { coded }\end{array}$ & $\begin{array}{c}\text { Temp } \\
\text { Setting }\end{array}$ & $\begin{array}{c}\text { Sensor } \\
\text { Depth }\end{array}$ & $\begin{array}{c}\text { Delta } \\
\text { Temp }\end{array}$ & $\begin{array}{c}\text { Measured } \\
\text { Temp. }\end{array}$ \\
\hline 59 & 67 & 2 & 3 & 600 & 36 & 7.8 & 607.8 \\
\hline 75 & 68 & 2 & 3 & 600 & 36 & 9.5 & 609.5 \\
\hline 27 & 75 & 2 & 3 & 600 & 36 & 8.3 & 608.3 \\
\hline 42 & 5 & 2 & 2 & 600 & 41 & 8.7 & 608.7 \\
\hline 66 & 10 & 1 & 2 & 585 & 41 & 10.1 & 595.1 \\
\hline 50 & 22 & 1 & 2 & 585 & 41 & 12.5 & 597.5 \\
\hline 18 & 27 & 1 & 2 & 585 & 41 & 8.5 & 593.5 \\
\hline 26 & 53 & 2 & 2 & 600 & 41 & 9.8 & 609.8 \\
\hline 74 & 55 & 2 & 2 & 600 & 41 & 9.9 & 609.9 \\
\hline 10 & 56 & 2 & 2 & 600 & 41 & 10.5 & 610.5 \\
\hline 2 & 57 & 1 & 2 & 585 & 41 & 10.0 & 595.0 \\
\hline 34 & 70 & 1 & 2 & 585 & 41 & 10.5 & 595.5 \\
\hline 58 & 79 & 2 & 2 & 600 & 41 & 11.4 & 611.4 \\
\hline 65 & 13 & 1 & 1 & 585 & 46 & 8.4 & 593.4 \\
\hline 17 & 32 & 1 & 1 & 585 & 46 & 6.8 & 591.8 \\
\hline 1 & 36 & 1 & 1 & 585 & 46 & 8.5 & 593.5 \\
\hline 57 & 43 & 2 & 1 & 600 & 46 & 5.1 & 605.1 \\
\hline 9 & 44 & 2 & 1 & 600 & 46 & 5.6 & 605.6 \\
\hline 73 & 45 & 2 & 1 & 600 & 46 & 6.5 & 606.5 \\
\hline 33 & 58 & 1 & 1 & 585 & 46 & 10.0 & 595.0 \\
\hline 25 & 62 & 2 & 1 & 600 & 46 & 9.7 & 609.7 \\
\hline 41 & 65 & 2 & 1 & 600 & 46 & 10.1 & 610.1 \\
\hline 49 & 74 & 1 & 1 & 585 & 46 & 9.5 & 594.5 \\
\hline
\end{tabular}

General Linear Model: Measured Temp versus Sensor Depth; Temperature Setting

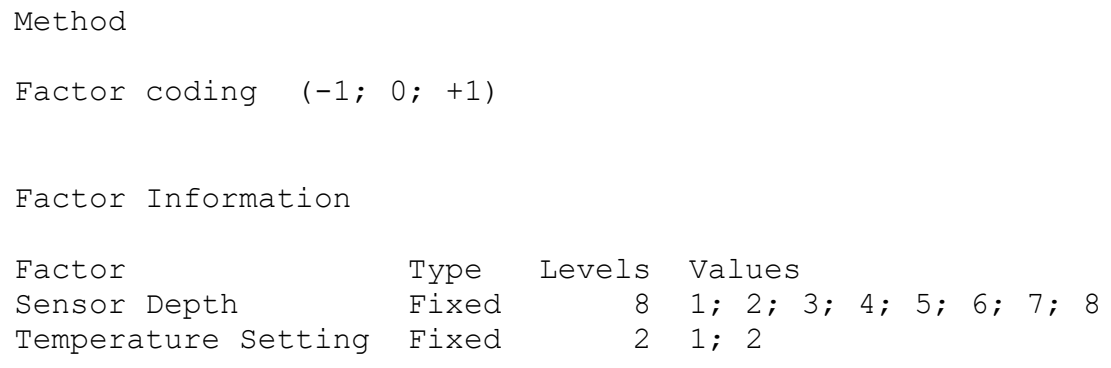




$\begin{array}{lrrrrr}\text { Analysis of Variance } & & & & & \\ \text { Source } & \text { DF } & \text { Adj SS } & \text { Adj MS } & \text { F-Value } & \text { P-Value } \\ \quad \text { Sensor Depth } & 7 & 85611.8 & 12230.3 & 543.36 & 0.000 \\ \quad \text { Temperature Setting } & 1 & 3759.3 & 3759.3 & 167.02 & 0.000 \\ \text { Error } & 71 & 1598.1 & 22.5 & & \\ \quad \text { Lack-of-Fit } & 7 & 183.3 & 26.2 & 1.18 & 0.324 \\ \quad \text { Pure Error } & 64 & 1414.8 & 22.1 & & \\ \text { Total } & 79 & 90969.1 & & & \end{array}$

Model Summary

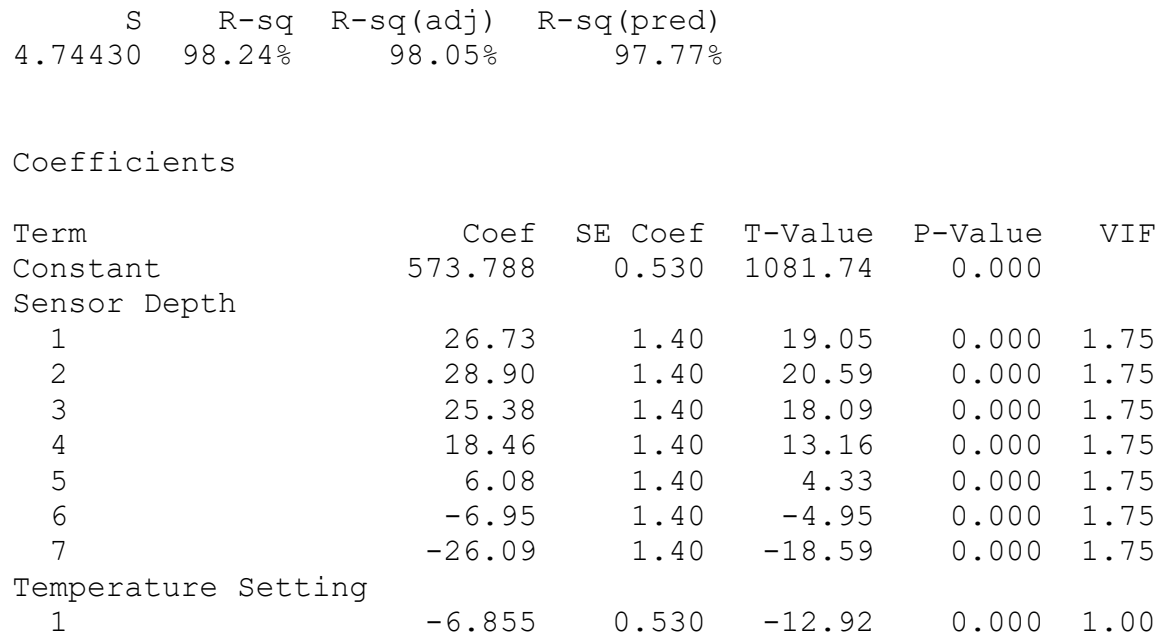

Regression Equation

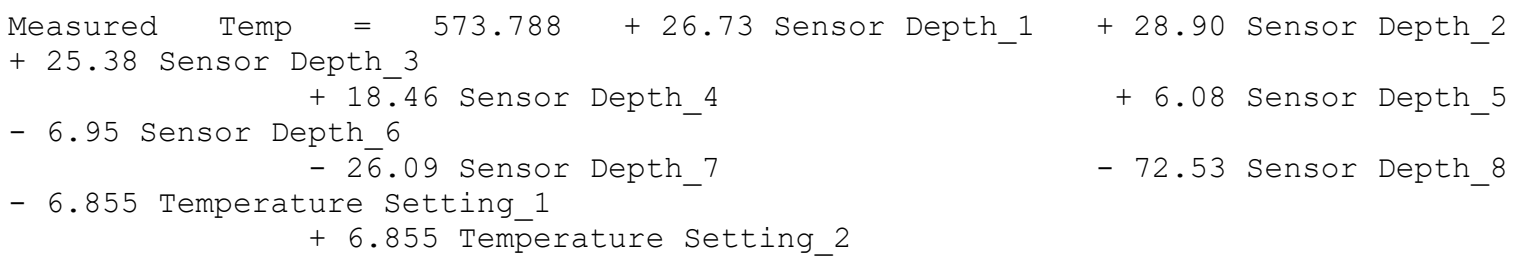




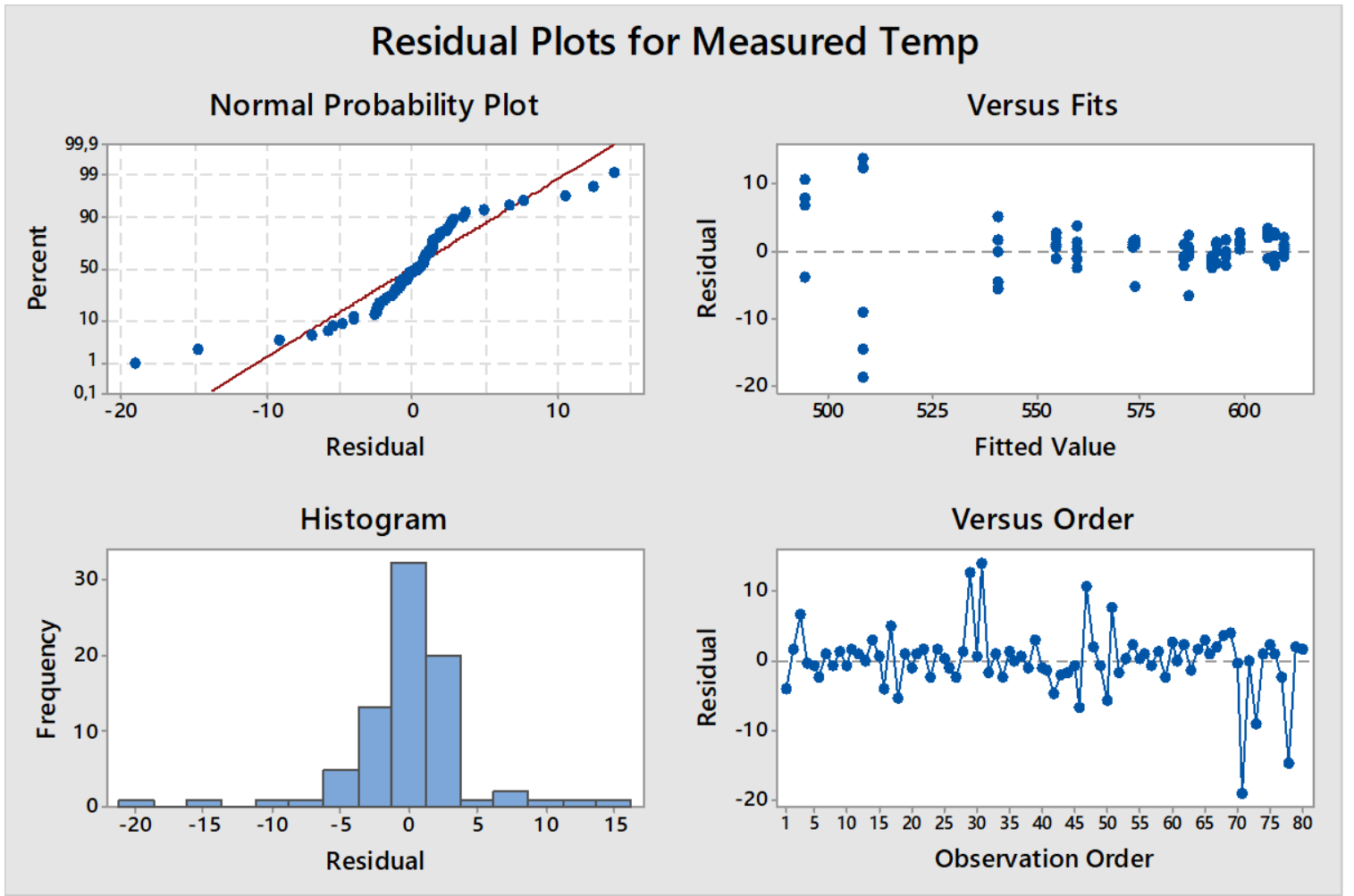

\section{Analysis with delta temperature}

Check for Blocking on Temperature setting:

\section{General Linear Model: Measured Temp Delta versus Sensor Depth; Temperature Setting}

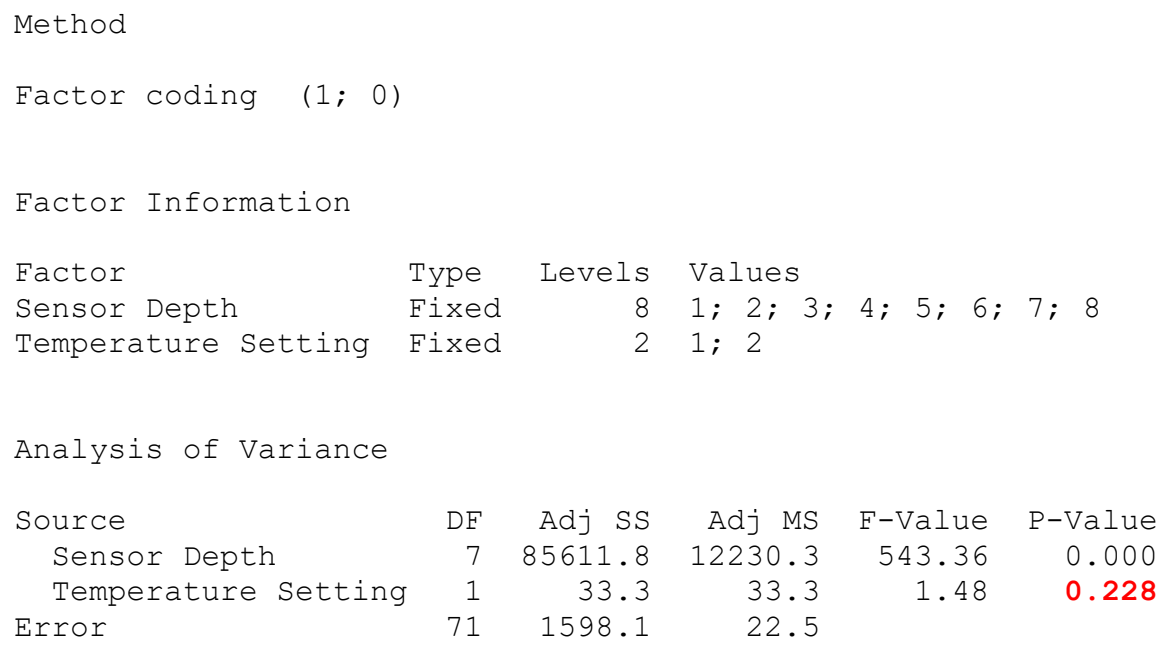




$\begin{array}{crrrrr}\text { Lack-of-Fit } & 7 & 183.3 & 26.2 & 1.18 & 0.324 \\ \text { Pure Error } & 64 & 1414.8 & 22.1 & & \\ \text { Total } & 79 & 87243.1 & & & \end{array}$

As $\mathrm{P}$ Value of Temperature Setting is $0.228>0.05$ blocking is not necessary.

\section{General Linear Model: Measured Temp Delta versus Sensor Depth}

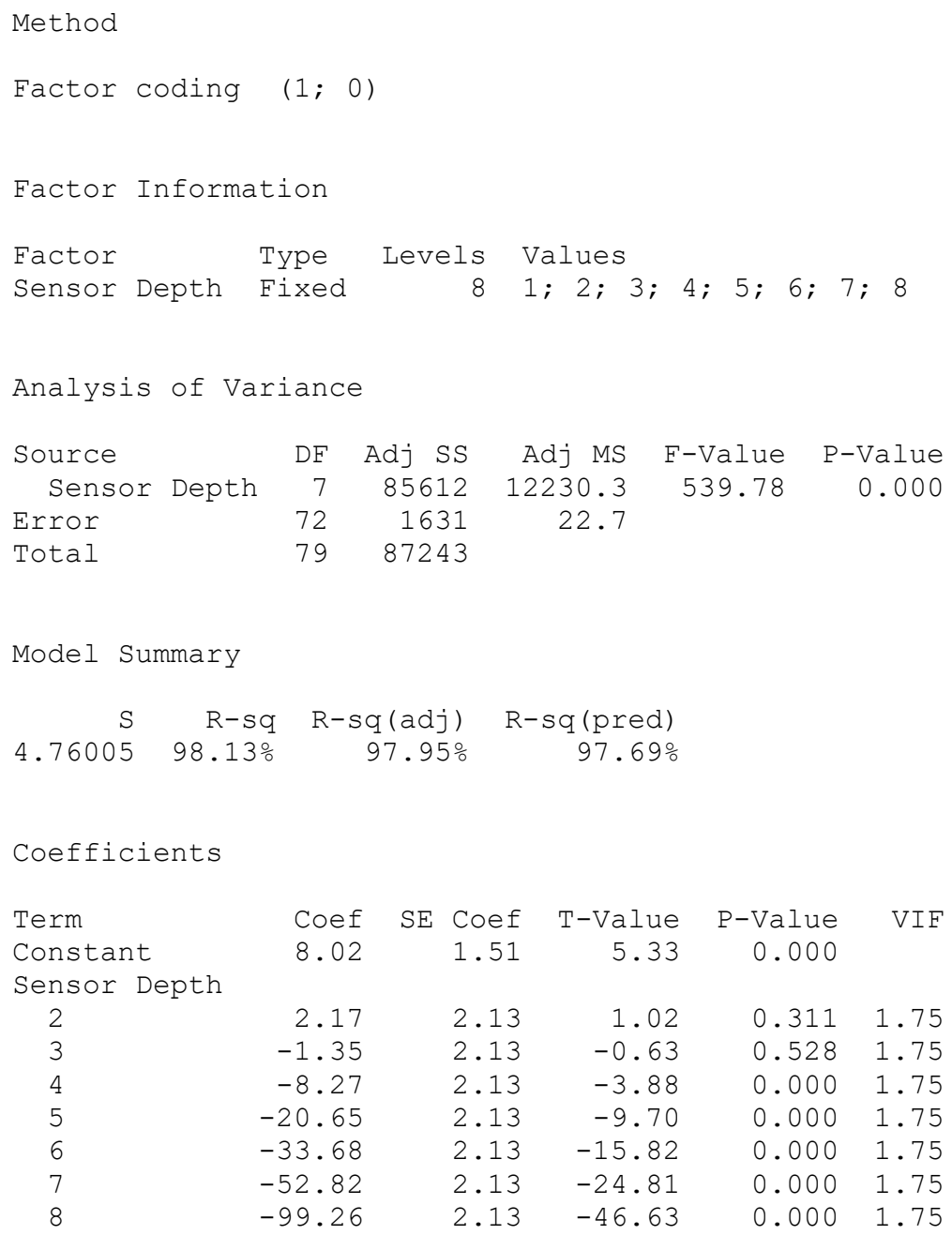



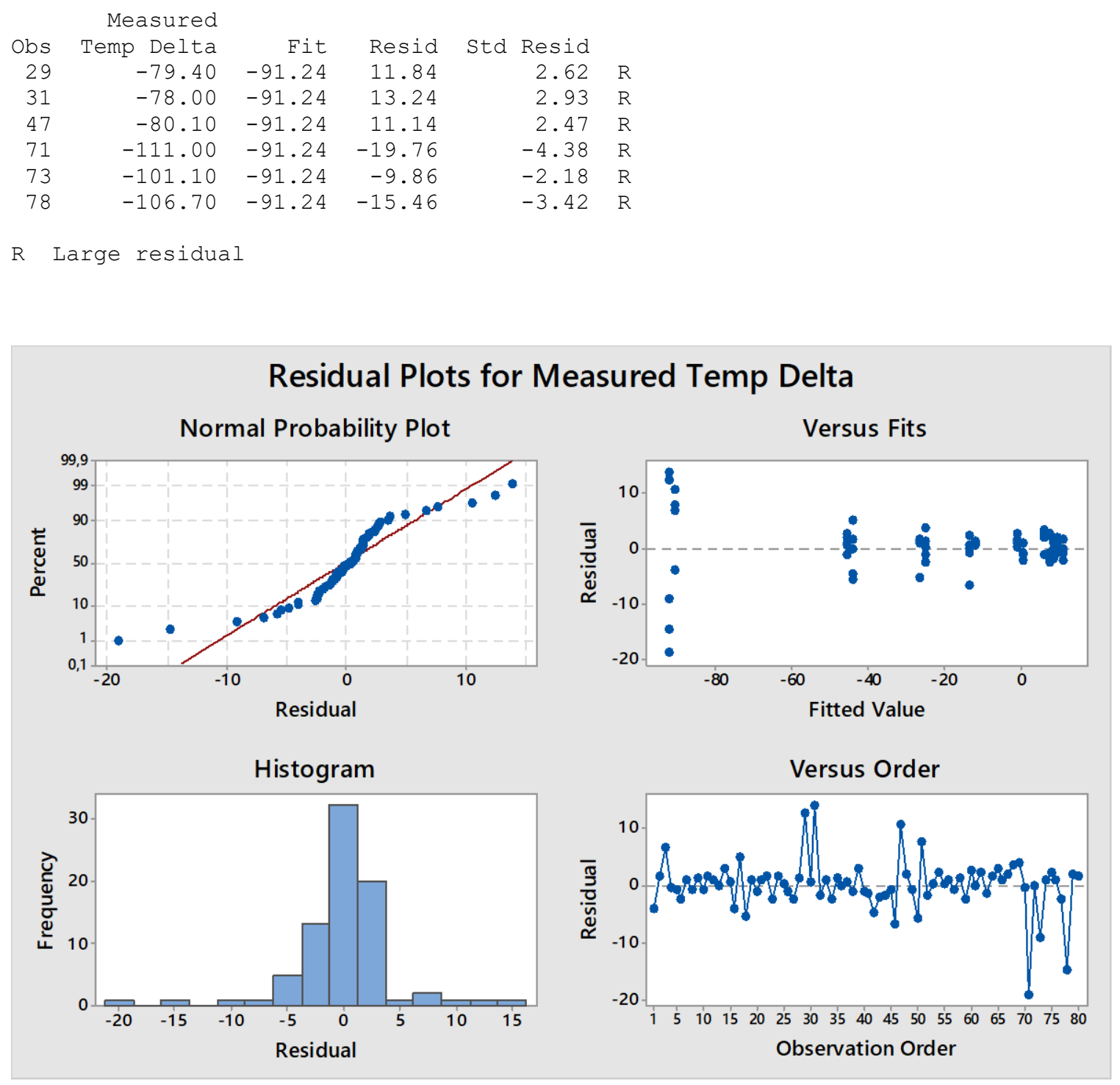

Overall inadequate linear model.

Repeat of Delta Analysis for semi solid range only with $\mathbf{3}$ unusual results removed:

General Linear Model: Delta Temp versus Sensor Depth

Method

Factor coding $(-1 ; 0 ;+1)$

Factor Information

\begin{tabular}{llrl} 
Factor & Type & Levels & Values \\
\hline Sensor Depth coded & Fixed & 6 & $1 ; 2 ; 3 ; 4 ; 5 ; 6$
\end{tabular}


Temperature Setting coded Fixed 2 1; 2

Analysis of Variance

\begin{tabular}{lrrrrr} 
Source & DF & Adj SS & Adj MS & F-Value & P-Value \\
\hline Sensor Depth coded & 5 & 9995.6 & 1999.12 & 555.97 & 0.000 \\
Temperature Setting coded & 1 & 3.5 & 3.46 & 0.96 & 0.332 \\
Sensor Depth codedTemperature Setting & 5 & 52.3 & 10.47 & 2.91 & 0.022 \\
coded & & & & & \\
Error & 48 & 172.6 & 3.60 & & \\
Total & 59 & 10224.0 & & &
\end{tabular}

\section{Model Summary}

\begin{tabular}{rrrr} 
S & R-sq & R-sq(adj) & R-sq(pred) \\
\hline 1.89625 & $98.31 \%$ & $97.92 \%$ & $97.36 \%$
\end{tabular}

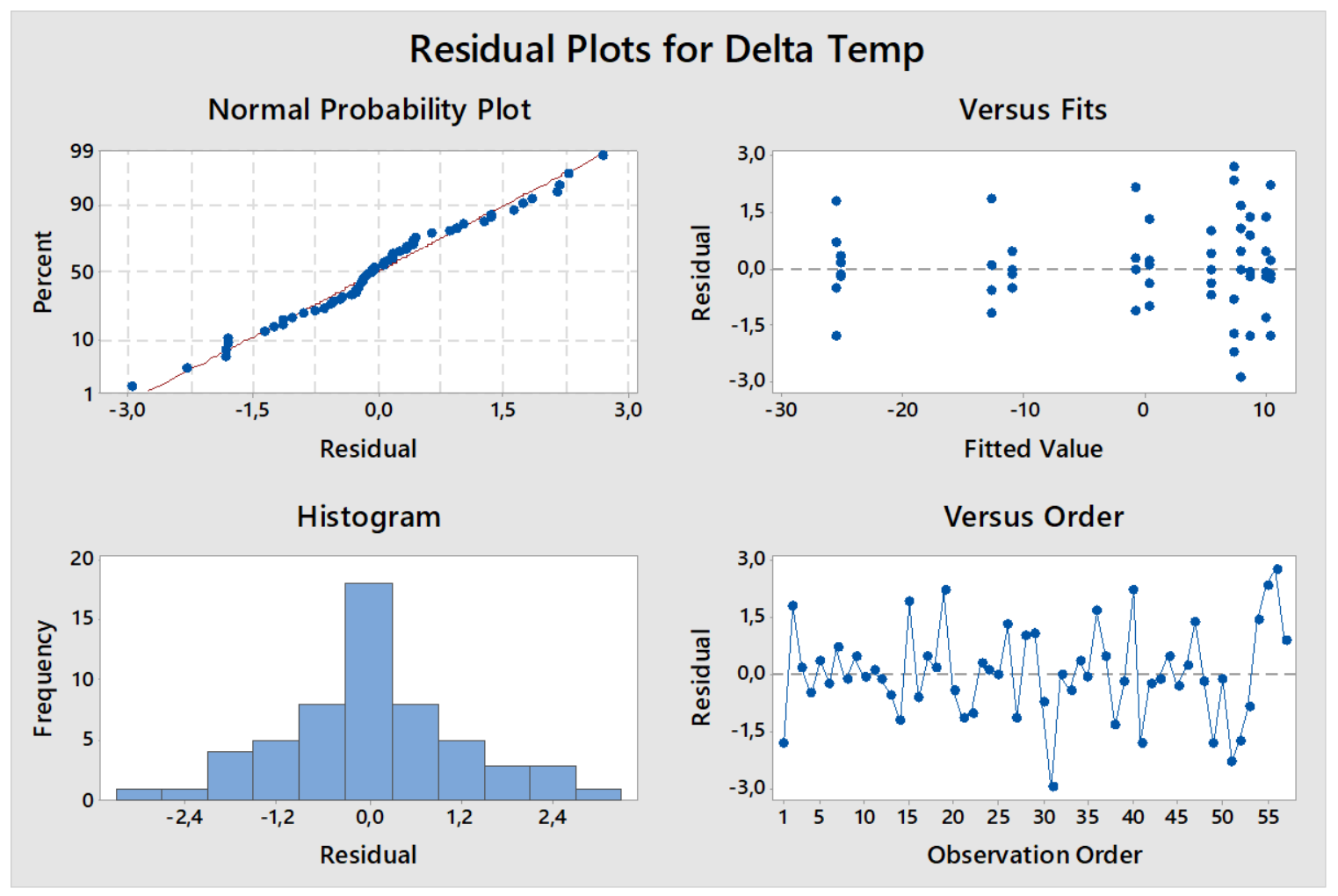




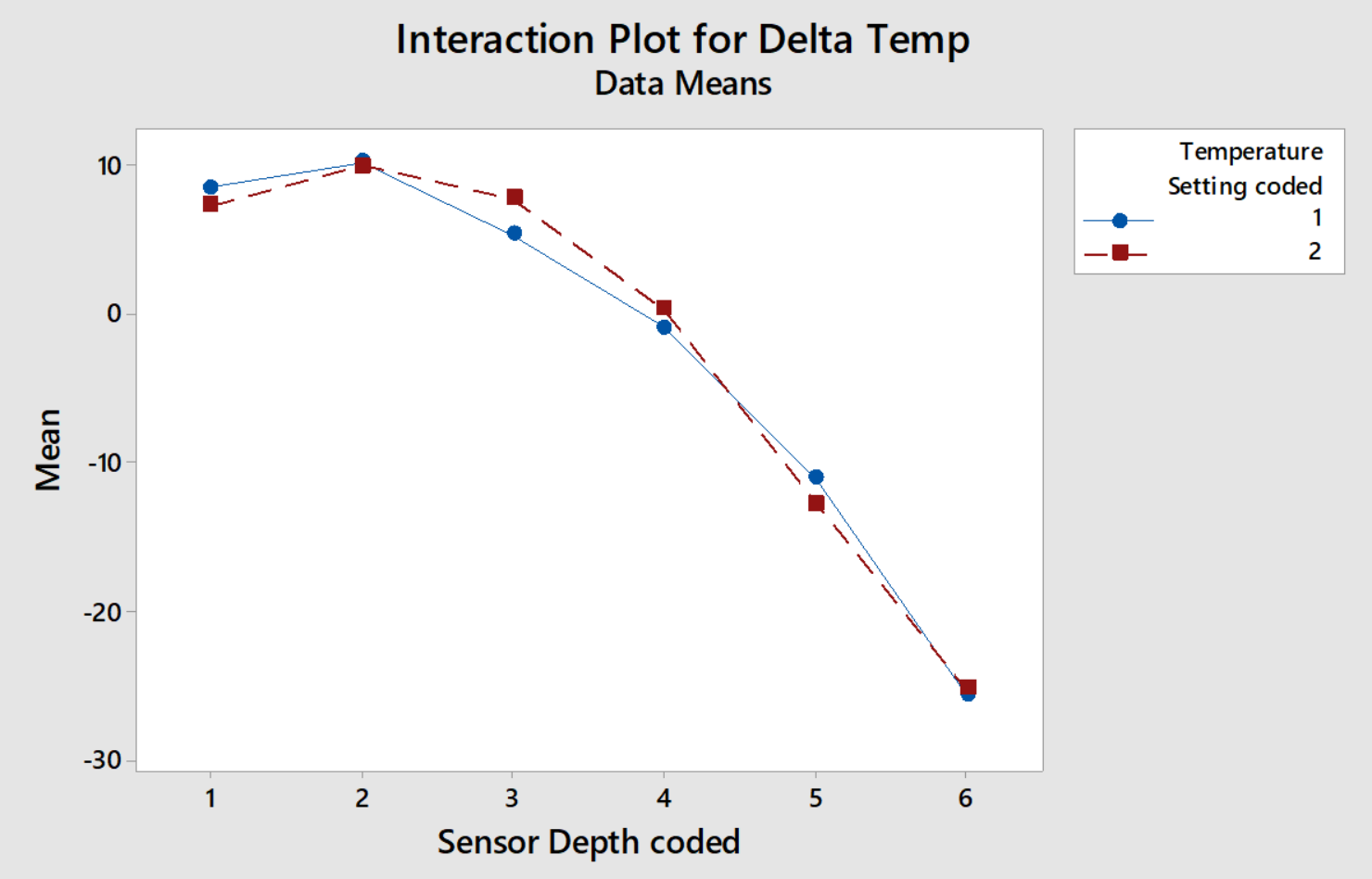




\section{Heating to globular structure experiment}

\section{Design of experiment}

\begin{tabular}{|c|c|c|c|c|c|c|c|}
\hline StdOrder & RunOrder & PtType & Blocks & Time & Temp & Alloy & Temp \\
\hline 44 & 1 & 1 & 1 & 20 Min & Low & THX540 & 595 \\
\hline 1 & 2 & 1 & 1 & $15 \mathrm{Min}$ & High & A356 & 595 \\
\hline 10 & 3 & 1 & 1 & 25 Min & High & THX540 & 605 \\
\hline 30 & 4 & 1 & 1 & $20 \mathrm{Min}$ & High & THX540 & 605 \\
\hline 35 & 5 & 1 & 1 & $25 \mathrm{Min}$ & Low & A356 & 585 \\
\hline 18 & 6 & 1 & 1 & 20 Min & High & THX540 & 605 \\
\hline 6 & 7 & 1 & 1 & 20 Min & High & THX540 & 605 \\
\hline 20 & 8 & 1 & 1 & 20 Min & Low & THX540 & 595 \\
\hline 24 & 9 & 1 & 1 & $25 \mathrm{Min}$ & Low & THX540 & 595 \\
\hline 4 & 10 & 1 & 1 & 15 Min & Low & THX540 & 595 \\
\hline 13 & 11 & 1 & 1 & 15 Min & High & A356 & 595 \\
\hline 43 & 12 & 1 & 1 & 20 Min & Low & A356 & 585 \\
\hline 29 & 13 & 1 & 1 & 20 Min & High & A356 & 595 \\
\hline 25 & 14 & 1 & 1 & $15 \mathrm{Min}$ & High & A356 & 595 \\
\hline 2 & 15 & 1 & 1 & 15 Min & High & THX540 & 605 \\
\hline 42 & 16 & 1 & 1 & 20 Min & High & THX540 & 605 \\
\hline 32 & 17 & 1 & 1 & 20 Min & Low & THX540 & 595 \\
\hline 3 & 18 & 1 & 1 & 15 Min & Low & A356 & 585 \\
\hline 34 & 19 & 1 & 1 & 25 Min & High & THX540 & 605 \\
\hline 11 & 20 & 1 & 1 & 25 Min & Low & A356 & 585 \\
\hline 38 & 21 & 1 & 1 & 15 Min & High & THX540 & 605 \\
\hline 26 & 22 & 1 & 1 & 15 Min & High & THX540 & 605 \\
\hline 27 & 23 & 1 & 1 & 15 Min & Low & A356 & 585 \\
\hline 15 & 24 & 1 & 1 & 15 Min & Low & A356 & 585 \\
\hline 23 & 25 & 1 & 1 & 25 Min & Low & A356 & 585 \\
\hline 19 & 26 & 1 & 1 & 20 Min & Low & A356 & 585 \\
\hline 48 & 27 & 1 & 1 & 25 Min & Low & THX540 & 595 \\
\hline 7 & 28 & 1 & 1 & 20 Min & Low & A356 & 585 \\
\hline 46 & 29 & 1 & 1 & $25 \mathrm{Min}$ & High & THX540 & 605 \\
\hline 14 & 30 & 1 & 1 & 15 Min & High & THX540 & 605 \\
\hline 39 & 31 & 1 & 1 & 15 Min & Low & A356 & 585 \\
\hline 31 & 32 & 1 & 1 & 20 Min & Low & A356 & 585 \\
\hline 41 & 33 & 1 & 1 & 20 Min & High & A356 & 595 \\
\hline 47 & 34 & 1 & 1 & 25 Min & Low & A356 & 585 \\
\hline 33 & 35 & 1 & 1 & 25 Min & High & A356 & 595 \\
\hline 9 & 36 & 1 & 1 & 25 Min & High & A356 & 595 \\
\hline 21 & 37 & 1 & 1 & 25 Min & High & A356 & 595 \\
\hline
\end{tabular}




\begin{tabular}{|r|r|r|r|l|l|l|r|}
\hline 28 & 38 & 1 & 1 & $15 \mathrm{Min}$ & Low & THX540 & 595 \\
\hline 36 & 39 & 1 & 1 & $25 \mathrm{Min}$ & Low & THX540 & 595 \\
\hline 8 & 40 & 1 & 1 & $20 \mathrm{Min}$ & Low & THX540 & 595 \\
\hline 17 & 41 & 1 & 1 & $20 \mathrm{Min}$ & High & A356 & 595 \\
\hline 12 & 42 & 1 & 1 & $25 \mathrm{Min}$ & Low & THX540 & 595 \\
\hline 37 & 43 & 1 & 1 & $15 \mathrm{Min}$ & High & A356 & 595 \\
\hline 45 & 44 & 1 & 1 & $25 \mathrm{Min}$ & High & A356 & 595 \\
\hline 5 & 45 & 1 & 1 & $20 \mathrm{Min}$ & High & A356 & 595 \\
\hline 40 & 46 & 1 & 1 & $15 \mathrm{Min}$ & Low & THX540 & 595 \\
\hline 16 & 47 & 1 & 1 & $15 \mathrm{Min}$ & Low & THX540 & 595 \\
\hline 22 & 48 & 1 & 1 & $25 \mathrm{Min}$ & High & THX540 & 605 \\
\hline
\end{tabular}

\section{Image manipulation process}

\section{Step overview:}

\begin{tabular}{|c|c|c|c|c|}
\hline Step & Name & Step by Step Guide & $\begin{array}{l}\text { Description / } \\
\text { Reason }\end{array}$ & Makros \\
\hline \multicolumn{5}{|c|}{ Image J } \\
\hline Opt 1 & Open Image & File -> Open & Open Original & \\
\hline Opt 2 & $\begin{array}{l}\text { Save } \\
\text { Processed } \\
\text { Image }\end{array}$ & File -> Save As & $\begin{array}{l}\text { Save Processed } \\
\text { Image }\end{array}$ & \\
\hline Opt 3 & $\begin{array}{l}\text { Re-Open } \\
\text { Original Image }\end{array}$ & $\begin{array}{l}\text { File -> Open (Original } \\
\text { Image) }\end{array}$ & & \\
\hline Opt 4 & Create Stack & $\begin{array}{l}\text { Image }->\text { Stacks }->\text { Image } \\
\text { to Stack }\end{array}$ & $\begin{array}{l}\text { Create Stack with } \\
\text { Original and } \\
\text { Processed Image }\end{array}$ & \\
\hline 1 & $\begin{array}{l}\text { Make } \\
\text { Rectangle } \\
2048 \times 1400\end{array}$ & & $\begin{array}{l}(0,0,2048,1400) \\
\text { Sets rectangle for } \\
\text { cropping the scale }\end{array}$ & \multirow{5}{*}{$\begin{array}{c}\text { Macro1- } \\
\text { ImagaProcessing } \\
\\
\text { Process -> } \\
\text { Multiple Image } \\
\text { Processor }\end{array}$} \\
\hline 2 & Crop & Image->Crop & $\begin{array}{l}\text { Crops the lower part } \\
\text { of the image with } \\
\text { the scale }\end{array}$ & \\
\hline 3 & Smoothen & Process $->$ Smooth & $\begin{array}{l}\text { Blurs the active } \\
\text { image or selection. } \\
\text { This filter replaces } \\
\text { each pixel with the } \\
\text { average of its } 3 \times 3 \\
\text { neighborhood. }\end{array}$ & \\
\hline 4 & Median Filter & $\begin{array}{l}\text { Process -> Filter -> } \\
\text { Median } \\
\text { Radius: } 4 \text { Pixel }\end{array}$ & Noise removal & \\
\hline 5 & 8 Bit Image & Image -> Type -> 8-bit & $\begin{array}{l}\text { Change image to } 8 \\
\text { bit for auto } \\
\text { Thresholding }\end{array}$ & \\
\hline
\end{tabular}




\begin{tabular}{|c|c|c|c|c|}
\hline 6 & $\begin{array}{l}\text { Auto Local } \\
\text { Thresholding }\end{array}$ & $\begin{array}{l}\text { Image -> Adjust -> Auto } \\
\text { local threshold } \\
\text { Method: Otsu } \\
\text { Radius: } 100 \\
\text { Optional: choose -> Stack }\end{array}$ & $\begin{array}{l}\text { Create black and } \\
\text { white picture } \\
\text { Optional: Add to } \\
\text { Stack }\end{array}$ & \\
\hline 7 & $\begin{array}{l}\text { Remove } \\
\text { Outliers }\end{array}$ & $\begin{array}{l}\text { Process -> Noise -> } \\
\text { Remove Outliers } \\
\text { Dark objects: Radius 5-10 } \\
\text { Bright objects: Radius 10- } \\
40\end{array}$ & $\begin{array}{l}\text { Remove Outliers } \\
\text { (black pores in } \\
\text { globules and white } \\
\text { pseudo- } \\
\text { particles/branches) }\end{array}$ & \multirow[t]{2}{*}{$\begin{array}{l}\text { Macro 2-Remove } \\
\text { Outliers 10dark- } \\
\text { 10bright } \\
\text { Process -> } \\
\text { Multiple Image } \\
\text { Processor }\end{array}$} \\
\hline 8 & $\begin{array}{l}\text { RGB Color } \\
\text { Image }\end{array}$ & $\begin{array}{l}\text { Image -> Type -> RGB } \\
\text { Color }\end{array}$ & $\begin{array}{l}\text { Change image to } \\
\text { Color for manual } \\
\text { globule separation }\end{array}$ & \\
\hline \multicolumn{5}{|c|}{ GIMP } \\
\hline 9 & $\begin{array}{l}\text { Open original } \\
\text { image }\end{array}$ & File -> Open & Open Original & \\
\hline 10 & $\begin{array}{l}\text { Brightness/ } \\
\text { Contrast }\end{array}$ & $\begin{array}{l}\text { Tools -> Color-> } \\
\text { brightness/contrast } \\
\text { Brightness: ca. }-30 \\
\text { Contrast: ca. }+100\end{array}$ & $\begin{array}{l}\text { Set high contrast } \\
\text { and low brightness } \\
\text { for better visibility } \\
\text { of globule outlines } \\
\text { in superimposed } \\
\text { image }\end{array}$ & \\
\hline 11 & $\begin{array}{l}\text { Open } \\
\text { processed } \\
\text { image }\end{array}$ & File -> Open & $\begin{array}{l}\text { Open processed } \\
\text { image }\end{array}$ & \\
\hline 12 & $\begin{array}{l}\text { Superimpose } \\
\text { images }\end{array}$ & $\begin{array}{l}\text { Processed image: Edit -> } \\
\text { copy visible } \\
\text { Original image: Edit-> } \\
\text { Paste as -> New Layer } \\
\text { Opacity of new Layer ca. } \\
30-50 \%\end{array}$ & $\begin{array}{l}\text { Superimpose } \\
\text { original and } \\
\text { processed image for } \\
\text { better } \\
\text { comparability }\end{array}$ & \\
\hline 13 & $\begin{array}{l}\text { Separate } \\
\text { globules }\end{array}$ & $\begin{array}{l}\text { Manual separation of } \\
\text { globules by drawing lines } \\
\text { with size }=5 \text { (preferred } \\
\text { color bright green) }\end{array}$ & $\begin{array}{l}\text { Color is chosen for } \\
\text { good contrast }\end{array}$ & \\
\hline 14 & $\begin{array}{l}\text { Delete original } \\
\text { image layer }\end{array}$ & & & \\
\hline 15 & Crop & Image-> Auto crop & $\begin{array}{l}\text { Crops image to } \\
2048 \times 1400\end{array}$ & \\
\hline 16 & $\begin{array}{l}\text { Save } \\
\text { separated } \\
\text { image }\end{array}$ & File -> Export as $->$ & $\begin{array}{l}\text { Saves the processed } \\
\text { image with } \\
\text { separated globules }\end{array}$ & \\
\hline \multicolumn{5}{|c|}{ Image J } \\
\hline 17 & $\begin{array}{l}\text { Length } \\
\text { calibration of } \\
\text { ImageJ }\end{array}$ & $\begin{array}{l}\text { Analyze }->\text { Set Scale: } \\
\text { known distance in pixels= } \\
305 \\
\text { known distance }=200 \\
\text { Unit of length }=\mu \mathrm{m}\end{array}$ & $\begin{array}{l}\text { The scale ratio was } \\
\text { determined by } \\
\text { analysis of an } \\
\text { original image }\end{array}$ & $\begin{array}{l}\text { Macro3- } \\
\text { ParticleAnalysis }\end{array}$ \\
\hline
\end{tabular}




\begin{tabular}{|c|c|c|c|c|}
\hline & & $\begin{array}{l}\text { Activate the checkbox for } \\
\text { global } \\
\text { Scale: } 1.525 \text { pixels/ } \mu \mathrm{m}\end{array}$ & $\begin{array}{l}\text { This step is } \\
\text { necessary after } \\
\text { every startup of } \\
\text { ImageJ, not for } \\
\text { every image }\end{array}$ & $\begin{array}{l}\text { Process -> } \\
\text { Multiple Image } \\
\text { Processor }\end{array}$ \\
\hline 18 & $\begin{array}{l}\text { Set } \\
\text { measurements }\end{array}$ & $\begin{array}{l}\text { Analyze -> Set } \\
\text { Measurements } \\
\text {-Area } \\
\text {-Shape descriptors } \\
\text {-Area fraction } \\
\text {-Perimeter }\end{array}$ & $\begin{array}{l}\text { Definition of output } \\
\text { parameters of } \\
\text { following analysis } \\
\text { This step is } \\
\text { necessary after } \\
\text { every startup of } \\
\text { ImageJ, not for } \\
\text { every image }\end{array}$ & \\
\hline 19 & $\begin{array}{l}8 \text { Bit Image } \\
\text { Thresholding } \\
\text { Inversion }\end{array}$ & $\begin{array}{l}\text { Image -> Type }->8 \text {-bit } \\
\text { Image -> Adjust -> } \\
\text { Threshold }(0,200) \\
\text { Edit -> Invert }\end{array}$ & $\begin{array}{l}\text { Change image to } 8 \\
\text { bit for Thresholding } \\
\text { Create black and } \\
\text { white picture, value } \\
\text { depends on chosen } \\
\text { color in step } 13 \\
\text { Inversion necessary } \\
\text { for Analyze particles }\end{array}$ & \\
\hline 20 & $\begin{array}{l}\text { Analyze } \\
\text { particles }\end{array}$ & $\begin{array}{l}\text { Analyze -> Analyze } \\
\text { Particles } \\
\text { Size: } 100 \text {-Infinity } \\
\text { Show: Outlines } \\
\text {-Display results } \\
\text {-Summarize } \\
\text {-Add to manager } \\
\text { (-include holes) }\end{array}$ & $\begin{array}{l}\text { If ,include holes' is } \\
\text { checked the results } \\
\text { show the effective } \\
\text { percentage of the } \\
\text { liquid phase. } \\
\text { Otherwise the } \\
\text { relative percentage } \\
\text { is shown }\end{array}$ & \\
\hline
\end{tabular}

\section{Macro1:}

makeRectangle(0, 0, 2048, 1400);

run("Crop");

run("Smooth");

run("Median...", "radius=4");

run("8-bit");

run("Auto Local Threshold", "method=Otsu radius=100 parameter_1=0 parameter_2=0 white");

\section{Macro 2:}


run("Remove Outliers...", "radius=10 threshold=50 which=Dark");

run("Remove Outliers...", "radius=10 threshold=50 which=Bright");

run("RGB Color");

\section{Macro 3:}

inputname=getTitle; // sets ShortFileName to file name without .jpg for renaming results StrInd1=indexOf(inputname, ".jpg");

ShortFileName=substring(inputname,0,StrInd1);

run("Set Scale...", "distance=305 known=200 pixel=1 unit=um global"); //sets scale for measurement setOption("BlackBackground", false);

run("8-bit"); //prepares image for particle analysis

setAutoThreshold("Default");

setThreshold(0, 200);

run("Convert to Mask");

run("Invert");

run("Set Measurements...", "area perimeter shape area_fraction display redirect=None decimal=3"); //sets parameters for output of particle analysis

selectWindow(inputname); // selects Image and starts first analysis for effective area fraction (holes/pores are ignored)

run("Analyze Particles...", "size=100-Infinity show=Outlines display clear include summarize add");

selectWindow("Results"); // selects results window and saves results saveAs("Results", "<Location>||Results||"+ShortFileName+"_includeHoles.tsv");

selectWindow(inputname); // selects Image and starts second analysis for relatice area fraction (holes/pores are taken into account)

run("Analyze Particles...", "size=100-Infinity show=Outlines display clear summarize add"); selectWindow("Results"); // selects results window and saves results saveAs("Results", "<Location>||Results||"ShortFileName+"_excludeHoles.tsv"); selectWindow(inputname); // selects Image and starts third analysis circularity (without particles on edges)

run("Analyze Particles...", "size=100-Infinity display exclude clear include summarize add"); selectWindow("Results"); // selects results window and saves results saveAs("Results", "<Location>||Results||"+ShortFileName+"_inHoles_exEdges.tsv"); selectWindow("Summary"); // selects summary window and saves results saveAs("Results", "<Location>||Results||"+ShortFileName+"_Summary_eff_real_circ.tsv"); 
A356 Rheocast Quality Index (RQI) results - Heating Experiment

\begin{tabular}{|c|c|c|c|c|c|c|c|c|c|c|c|c|c|}
\hline $\begin{array}{l}\text { Temp } \\
\text { setting }\end{array}$ & $\begin{array}{c}\text { Hold } \\
\text { Time in } \\
\text { min }\end{array}$ & $\begin{array}{c}\text { Run } \\
\text { Number }\end{array}$ & $\begin{array}{c}\text { Model } \\
\text { Temperature }\end{array}$ & FS & $\begin{array}{c}\text { Number } \\
\text { of } \\
\text { Grains }\end{array}$ & Area & Perimeter & $\begin{array}{l}\text { Shape } \\
\text { Factor }\end{array}$ & Diameter & GS & GLS & GS/GLS & RQI \\
\hline 595 & 15 & 2 & 582 & 71.227 & 66 & 8198.239 & 412.350 & 1.65 & 79.53 & 10.35 & 11.45 & 0.90 & 0.547688 \\
\hline 595 & 15 & 2 & 595 & 64.908 & 62 & 7696.481 & 367.653 & 1.40 & 83.74 & 9.50 & 9.90 & 0.96 & 0.686617 \\
\hline 595 & 15 & 2 & 592 & 59.311 & 41 & 12720.724 & 480.708 & 1.45 & 105.85 & 7.50 & 8.10 & 0.93 & 0.640524 \\
\hline 595 & 15 & 11 & 595 & 71.962 & 75 & 7084.599 & 359.288 & 1.45 & 78.87 & 10.15 & 10.65 & 0.95 & 0.657289 \\
\hline 595 & 15 & 11 & 592 & 73.893 & 48 & 12141.351 & 454.501 & 1.35 & 106.85 & 10.15 & 10.65 & 0.95 & 0.703921 \\
\hline 595 & 15 & 14 & 582 & 71.859 & 72 & 7918.54 & 413.113 & 1.72 & 76.67 & 11.35 & 13.25 & 0.86 & 0.499456 \\
\hline 595 & 15 & 14 & 595 & 72.868 & 91 & 7193.056 & 356.719 & 1.41 & 80.66 & 11.15 & 11.75 & 0.95 & \begin{tabular}{|l|}
0.674074 \\
\end{tabular} \\
\hline 595 & 15 & 14 & 592 & 56.583 & 52 & 9447.402 & 410.411 & 1.42 & 92.08 & 7.10 & 8.30 & 0.86 & 0.602927 \\
\hline 595 & 15 & 43 & 595 & 73.278 & 93 & 6833.913 & 346.348 & 1.40 & 78.93 & 11.70 & 12.40 & 0.94 & 0.675489 \\
\hline 595 & 15 & 43 & 592 & 68.406 & 71 & 8056.838 & 389.737 & 1.50 & 82.69 & 10.00 & 10.90 & 0.92 & 0.611512 \\
\hline 585 & 15 & 18 & 573 & 76.734 & 61 & 9494.525 & 429.110 & 1.54 & 88.50 & 10.25 & 11.25 & 0.91 & 0.59036 \\
\hline 585 & 15 & 18 & 585 & 72.895 & 82 & 7544.177 & 353.874 & 1.32 & 85.28 & 10.45 & 11.95 & 0.87 & 0.662023 \\
\hline 585 & 15 & 18 & 582 & 73.561 & 51 & 10513.767 & 442.178 & 1.48 & 95.11 & 9.60 & 11.50 & 0.83 & 0.564089 \\
\hline 585 & 15 & 23 & 573 & 70.848 & 129 & 4972.937 & 280.049 & 1.26 & 71.03 & 13.95 & 14.85 & 0.94 & 0.748518 \\
\hline 585 & 15 & 23 & 585 & 66.38 & 51 & 10174.048 & 438.633 & 1.50 & 92.78 & 9.95 & 11.05 & 0.90 & 0.598359 \\
\hline 585 & 15 & 23 & 582 & 65.937 & 58 & 8296.902 & 408.232 & 1.60 & 81.30 & 9.90 & 10.90 & 0.91 & 0.568225 \\
\hline 585 & 15 & 24 & 585 & 75.648 & 102 & 6878.729 & 348.019 & 1.40 & 79.06 & 11.40 & 12.30 & 0.93 & 0.661473 \\
\hline 585 & 15 & 24 & 582 & 78.224 & 54 & 10501.064 & 418.207 & 1.33 & 100.44 & 9.70 & 13.50 & 0.72 & 0.542124 \\
\hline 585 & 15 & 31 & 573 & 69.096 & 73 & 7737.54 & 400.026 & 1.65 & 77.37 & 11.05 & 11.95 & 0.92 & 0.561863 \\
\hline 585 & 15 & 31 & 585 & 67.016 & 58 & 8763.851 & 422.180 & 1.62 & 83.03 & 10.10 & 11.40 & 0.89 & 0.547427 \\
\hline 585 & 15 & 31 & 582 & 70.547 & 50 & 10824.239 & 460.430 & 1.56 & 94.04 & 9.55 & 10.85 & 0.88 & 0.564747 \\
\hline 595 & 20 & 13 & 582 & 70.444 & 94 & 6006.923 & 343.340 & 1.56 & 69.98 & 12.30 & 12.70 & 0.97 & 0.620175 \\
\hline
\end{tabular}




\begin{tabular}{|c|c|c|c|c|c|c|c|c|c|c|c|c|c|}
\hline $\begin{array}{l}\text { Temp } \\
\text { setting }\end{array}$ & $\begin{array}{l}\text { Hold } \\
\text { Time in } \\
\text { min }\end{array}$ & $\begin{array}{c}\text { Run } \\
\text { Number }\end{array}$ & $\begin{array}{c}\text { Model } \\
\text { Temperature }\end{array}$ & FS & $\begin{array}{c}\text { Number } \\
\text { of } \\
\text { Grains }\end{array}$ & Area & Perimeter & $\begin{array}{l}\text { Shape } \\
\text { Factor }\end{array}$ & Diameter & GS & GLS & GS/GLS & RQI \\
\hline 595 & 20 & 13 & 595 & 63.398 & 80 & 7186.428 & 349.691 & 1.35 & 82.20 & 9.60 & 10.40 & 0.92 & 0.681698 \\
\hline 595 & 20 & 13 & 592 & 59.572 & 54 & 9998.395 & 445.444 & 1.58 & 89.78 & 9.55 & 10.25 & 0.93 & 0.589975 \\
\hline 595 & 20 & 33 & 582 & 71.889 & 53 & 7995.684 & 384.502 & 1.47 & 83.18 & 10.65 & 11.75 & 0.91 & 0.615999 \\
\hline 595 & 20 & 33 & 595 & 71.604 & 80 & 7258.226 & 345.138 & 1.31 & 84.12 & 9.60 & 10.90 & 0.88 & 0.674372 \\
\hline 595 & 20 & 33 & 592 & 70.475 & 53 & 10198.419 & 427.453 & 1.43 & 95.43 & 9.05 & 11.25 & 0.80 & 0.564238 \\
\hline 595 & 20 & 41 & 582 & 76.708 & 113 & 6810.528 & 340.981 & 1.36 & 79.89 & 12.10 & 13.25 & 0.91 & 0.672202 \\
\hline 595 & 20 & 41 & 595 & 69.861 & 76 & 8442.914 & 372.561 & 1.31 & 90.65 & 10.30 & 10.60 & 0.97 & 0.742743 \\
\hline 595 & 20 & 41 & 592 & 61.302 & 67 & 6341.341 & 319.152 & 1.28 & 79.48 & 8.50 & 10.00 & 0.85 & 0.664989 \\
\hline 595 & 20 & 45 & 582 & 63.824 & 73 & 8101.095 & 370.799 & 1.35 & 87.39 & 9.50 & 9.80 & 0.97 & 0.717751 \\
\hline 595 & 20 & 45 & 595 & 65.944 & 76 & 7692.420 & 359.034 & 1.33 & 85.70 & 9.50 & 9.90 & 0.96 & 0.719598 \\
\hline 595 & 20 & 45 & 592 & 61.649 & 54 & 9548.927 & 423.002 & 1.49 & 90.30 & 8.80 & 9.50 & 0.93 & 0.62121 \\
\hline 585 & 20 & 12 & 573 & 79.61 & 63 & 9474.784 & 424.108 & 1.51 & 89.36 & 9.55 & 10.75 & 0.89 & 0.588059 \\
\hline 585 & 20 & 12 & 585 & 72.183 & 68 & 9108.235 & 391.260 & 1.34 & 93.12 & 9.55 & 10.85 & 0.88 & 0.658092 \\
\hline 585 & 20 & 12 & 582 & 66.358 & 49 & 10764.366 & 439.338 & 1.43 & 98.01 & 8.65 & 9.05 & 0.96 & 0.669836 \\
\hline 585 & 20 & 26 & 585 & 82.973 & 87 & 8748.072 & 391.374 & 1.39 & 89.41 & 12.90 & 14.10 & 0.91 & 0.656612 \\
\hline 585 & 20 & 28 & 573 & 76.896 & 78 & 9387.275 & 391.364 & 1.30 & 95.94 & 11.60 & 12.80 & 0.91 & 0.697968 \\
\hline 585 & 20 & 28 & 585 & 74.274 & 48 & 10518.642 & 407.261 & 1.25 & 103.31 & 8.00 & 9.30 & 0.86 & 0.685537 \\
\hline 585 & 20 & 28 & 582 & 77.324 & 45 & 12311.367 & 459.351 & 1.36 & 107.21 & 8.50 & 10.60 & 0.80 & 0.587949 \\
\hline 585 & 20 & 32 & 573 & 72.218 & 76 & 8536.087 & 356.399 & 1.18 & 95.80 & 9.95 & 10.55 & 0.94 & 0.796464 \\
\hline 585 & 20 & 32 & 585 & 69.144 & 75 & 7813.917 & 356.832 & 1.30 & 87.59 & 9.05 & 10.45 & 0.87 & 0.667857 \\
\hline 585 & 20 & 32 & 582 & 69.69 & 70 & 8879.702 & 365.044 & 1.19 & 97.30 & 9.30 & 10.30 & 0.90 & 0.756073 \\
\hline 595 & 25 & 35 & 582 & 76.33 & 51 & 12595.509 & 465.078 & 1.37 & 108.33 & 9.75 & 10.55 & 0.92 & 0.676279 \\
\hline 595 & 25 & 35 & 595 & 68.349 & 51 & 9416.292 & 377.596 & 1.20 & 99.75 & 7.70 & 9.00 & 0.86 & 0.710041 \\
\hline 595 & 25 & 35 & 592 & 71.986 & 53 & 9280.354 & 382.923 & 1.26 & 96.94 & 9.10 & 9.60 & 0.95 & 0.753914 \\
\hline
\end{tabular}




\begin{tabular}{|c|c|c|c|c|c|c|c|c|c|c|c|c|c|}
\hline $\begin{array}{l}\text { Temp } \\
\text { setting }\end{array}$ & $\begin{array}{c}\text { Hold } \\
\text { Time in } \\
\text { min }\end{array}$ & $\begin{array}{c}\text { Run } \\
\text { Number }\end{array}$ & $\begin{array}{c}\text { Model } \\
\text { Temperature }\end{array}$ & FS & $\begin{array}{c}\text { Number } \\
\text { of } \\
\text { Grains }\end{array}$ & Area & Perimeter & $\begin{array}{l}\text { Shape } \\
\text { Factor }\end{array}$ & Diameter & GS & GLS & GS/GLS & RQI \\
\hline 595 & 25 & 36 & 582 & 74.001 & 75 & 7754.492 & 346.481 & 1.23 & 89.52 & 11.05 & 11.55 & 0.96 & 0.776577 \\
\hline 595 & 25 & 36 & 595 & 72.792 & 71 & 7750.956 & 361.212 & 1.34 & 85.83 & 11.05 & 11.45 & 0.97 & 0.72044 \\
\hline 595 & 25 & 36 & 592 & 71.385 & 45 & 11406.883 & 449.984 & 1.41 & 101.40 & 8.20 & 9.30 & 0.88 & 0.624185 \\
\hline 595 & 25 & 37 & 582 & 75.768 & 67 & 8315.749 & 373.966 & 1.34 & 88.95 & 9.25 & 10.25 & 0.90 & 0.674318 \\
\hline 595 & 25 & 37 & 595 & 71.483 & 57 & 11074.978 & 427.513 & 1.31 & 103.62 & 9.30 & 9.70 & 0.96 & 0.730071 \\
\hline 595 & 25 & 37 & 592 & 71.164 & 50 & 11506.713 & 444.459 & 1.37 & 103.56 & 8.85 & 9.25 & 0.96 & 0.700324 \\
\hline 595 & 25 & 44 & 582 & 72.979 & 77 & 7820.224 & 354.772 & 1.28 & 88.17 & 11.00 & 11.30 & 0.97 & 0.760054 \\
\hline 595 & 25 & 44 & 595 & 67.172 & 60 & 10108.508 & 405.533 & 1.29 & 99.71 & 9.15 & 9.25 & 0.99 & 0.764054 \\
\hline 595 & 25 & 44 & 592 & 68.786 & 52 & 10345.515 & 419.908 & 1.36 & 98.55 & 9.30 & 9.90 & 0.94 & 0.69263 \\
\hline 585 & 25 & 5 & 573 & 65.082 & 71 & 7457.429 & 316.679 & 1.07 & 94.20 & 8.35 & 9.55 & 0.87 & 0.81704 \\
\hline 585 & 25 & 5 & 585 & 68.217 & 49 & 11740.877 & 468.131 & 1.49 & 100.32 & 8.80 & 9.20 & 0.96 & 0.643977 \\
\hline 585 & 25 & 5 & 582 & 53.501 & 38 & 11309.127 & 477.936 & 1.61 & 94.65 & 7.65 & 8.35 & 0.92 & 0.569999 \\
\hline 585 & 25 & 20 & 573 & 74.533 & 52 & 10847.977 & 435.886 & 1.39 & 99.55 & 8.55 & 9.75 & 0.88 & 0.629179 \\
\hline 585 & 25 & 20 & 585 & 70.362 & 50 & 11700.278 & 442.476 & 1.33 & 105.77 & 7.90 & 8.00 & 0.99 & 0.74159 \\
\hline 585 & 25 & 20 & 582 & 64.164 & 45 & 11937.513 & 502.865 & 1.69 & 94.96 & 8.25 & 8.65 & 0.95 & 0.565795 \\
\hline 585 & 25 & 25 & 573 & 72.101 & 146 & 4520.788 & 233.95 & 1.00 & 77.29 & 13.00 & 13.20 & 0.98 & 0.984848 \\
\hline 585 & 25 & 25 & 585 & 60.871 & 84 & 6381.649 & 309.671 & 1.20 & 82.43 & 10.10 & 10.20 & 0.99 & 0.828062 \\
\hline 585 & 25 & 25 & 582 & 60.489 & 92 & 6161.738 & 302.18 & 1.18 & 81.56 & 9.60 & 9.90 & 0.97 & 0.822276 \\
\hline 585 & 25 & 34 & 585 & 76.768 & 31 & 18382.488 & 602.026 & 1.57 & 122.14 & 7.85 & 10.65 & 0.74 & 0.46979 \\
\hline
\end{tabular}


THIXALLOY 540 Rheocast Quality Index (RQI) results - Heating Experiment

\begin{tabular}{|c|c|c|c|c|c|c|c|c|c|c|c|c|c|}
\hline $\begin{array}{l}\text { Temp } \\
\text { setting }\end{array}$ & $\begin{array}{c}\text { Hold } \\
\text { Time in } \\
\text { min }\end{array}$ & $\begin{array}{c}\text { Run } \\
\text { Number }\end{array}$ & $\begin{array}{c}\text { Model } \\
\text { Temperature }\end{array}$ & FS & $\begin{array}{c}\text { Number } \\
\text { of } \\
\text { Grains }\end{array}$ & Area & Perimeter & $\begin{array}{l}\text { Shape } \\
\text { Factor }\end{array}$ & Diameter & GS & GLS & GS/GLS & RQI \\
\hline 605 & 15 & 15 & 593 & 68.984 & 124 & 5038.021 & 301.73 & 1.44 & 66.79 & 12.45 & 12.85 & 0.97 & 0.67375 \\
\hline 605 & 15 & 15 & 605 & 62.502 & 132 & 4330.439 & 265.455 & 1.29 & 65.25 & 12.45 & 13.35 & 0.93 & 0.720192 \\
\hline 605 & 15 & 15 & 602 & 60.453 & 88 & 5633.197 & 309.445 & 1.35 & 72.82 & 9.55 & 9.95 & 0.96 & 0.709542 \\
\hline 605 & 15 & 21 & 605 & 56.799 & 98 & 5137.193 & 280.23 & 1.22 & 73.33 & 10.00 & 10.50 & 0.95 & 0.78292 \\
\hline 605 & 15 & 22 & 593 & 61.711 & 91 & 5362.245 & 303.539 & 1.37 & 70.66 & 11.75 & 12.75 & 0.92 & 0.673993 \\
\hline 605 & 15 & 22 & 605 & 57.727 & 86 & 5908.654 & 332.632 & 1.49 & 71.05 & 9.90 & 10.60 & 0.93 & 0.626758 \\
\hline 605 & 15 & 22 & 602 & 57.687 & 85 & 5498.787 & 305.257 & 1.35 & 72.05 & 10.05 & 10.75 & 0.93 & 0.693271 \\
\hline 605 & 15 & 30 & 593 & 59.747 & 94 & 6137.555 & 317.585 & 1.31 & 77.30 & 9.15 & 9.75 & 0.94 & 0.717632 \\
\hline 605 & 15 & 30 & 605 & 60.498 & 89 & 5730.626 & 313.529 & 1.37 & 73.11 & 9.65 & 10.15 & 0.95 & 0.696495 \\
\hline 605 & 15 & 30 & 602 & 58.323 & 56 & 7644.082 & 378.316 & 1.49 & 80.82 & 9.35 & 11.35 & 0.82 & 0.552894 \\
\hline 605 & 20 & 4 & 593 & 73.46 & 101 & 5343.48 & 318.842 & 1.51 & 67.04 & 10.70 & 11.60 & 0.92 & 0.609269 \\
\hline 605 & 20 & 4 & 605 & 65.523 & 78 & 6216.046 & 333.337 & 1.42 & 74.59 & 10.60 & 12.30 & 0.86 & 0.60584 \\
\hline 605 & 20 & 4 & 602 & 80.742 & 51 & 13029.58 & 492.268 & 1.48 & 105.87 & 10.35 & 10.95 & 0.95 & 0.638651 \\
\hline 605 & 20 & 6 & 593 & 64.958 & 110 & 5463.626 & 303.481 & 1.34 & 72.01 & 12.35 & 13.05 & 0.95 & 0.705479 \\
\hline 605 & 20 & 6 & 605 & 60.541 & 113 & 5186.882 & 291.442 & 1.30 & 71.19 & 11.10 & 11.60 & 0.96 & 0.734306 \\
\hline 605 & 20 & 6 & 602 & 63.135 & 76 & 6828.08 & 338.599 & 1.34 & 80.66 & 8.80 & 10.10 & 0.87 & 0.652076 \\
\hline 605 & 20 & 7 & 593 & 66.316 & 110 & 5215.763 & 268.907 & 1.10 & 77.58 & 10.95 & 11.65 & 0.94 & 0.851945 \\
\hline 605 & 20 & 7 & 605 & 52.827 & 92 & 5435.168 & 282.770 & 1.17 & 76.88 & 8.95 & 9.25 & 0.97 & 0.82649 \\
\hline 605 & 20 & 7 & 602 & 46.665 & 71 & 6198.303 & 307.404 & 1.21 & 80.65 & 8.00 & 8.40 & 0.95 & 0.785008 \\
\hline 605 & 20 & 16 & 593 & 56.279 & 68 & 7776.328 & 367.856 & 1.38 & 84.56 & 8.50 & 9.80 & 0.87 & 0.626357 \\
\hline 605 & 20 & 16 & 605 & 57.132 & 86 & 5797.531 & 297.544 & 1.22 & 77.94 & 8.95 & 10.15 & 0.88 & 0.725617 \\
\hline 605 & 20 & 16 & 602 & 53.187 & 74 & 6626.983 & 324.902 & 1.27 & 81.59 & 8.55 & 9.35 & 0.91 & 0.721399 \\
\hline 605 & 25 & 3 & 593 & 62.685 & 92 & 6115.514 & 324.179 & 1.37 & 75.46 & 12.20 & 12.50 & 0.98 & 0.713712 \\
\hline
\end{tabular}




\begin{tabular}{|c|c|c|c|c|c|c|c|c|c|c|c|c|c|c|}
\hline $\begin{array}{c}\text { Temp } \\
\text { setting }\end{array}$ & $\begin{array}{c}\text { Hold } \\
\text { Time in } \\
\text { min }\end{array}$ & $\begin{array}{c}\text { Run } \\
\text { Number }\end{array}$ & $\begin{array}{c}\text { Model } \\
\text { Temperature }\end{array}$ & FS & $\begin{array}{c}\text { Number } \\
\text { of } \\
\text { Grains }\end{array}$ & Area & Perimeter & $\begin{array}{c}\text { Shape } \\
\text { Factor }\end{array}$ & Diameter & GS & GLS & GS/GLS & RQI \\
\hline 605 & 25 & 3 & 605 & 63.922 & 102 & 6101.375 & 319.267 & 1.33 & 76.44 & 11.95 & 12.75 & 0.94 & 0.704997 \\
\hline 605 & 25 & 3 & 602 & 60.088 & 75 & 6491.548 & 310.975 & 1.19 & 83.50 & 9.20 & 9.50 & 0.97 & 0.816905 \\
\hline 605 & 25 & 19 & 593 & 63.551 & 83 & 6650.172 & 317.912 & 1.21 & 83.67 & 10.15 & 10.55 & 0.96 & 0.795504 \\
\hline 605 & 25 & 19 & 605 & 64.277 & 119 & 5115.014 & 281.602 & 1.23 & 72.66 & 11.10 & 11.70 & 0.95 & 0.768993 \\
\hline 605 & 25 & 19 & 602 & 55.81 & 86 & 5942.689 & 291.708 & 1.14 & 81.49 & 8.40 & 9.00 & 0.93 & 0.819093 \\
\hline 605 & 25 & 29 & 593 & 62.514 & 96 & 6195.436 & 310.814 & 1.24 & 79.73 & 11.40 & 11.60 & 0.98 & 0.792004 \\
\hline 605 & 25 & 29 & 605 & 61.727 & 74 & 7094.42 & 332.859 & 1.24 & 85.25 & 9.15 & 9.65 & 0.95 & 0.762957 \\
\hline 605 & 25 & 29 & 602 & 57.228 & 59 & 7949.99 & 368.585 & 1.36 & 86.28 & 8.65 & 9.05 & 0.96 & 0.70286 \\
\hline 605 & 25 & 48 & 593 & 76.723 & 141 & 5193.36 & 289.37 & 1.28 & 71.79 & 13.45 & 13.55 & 0.99 & 0.773632 \\
\hline 605 & 25 & 48 & 605 & 69.76 & 115 & 5849.225 & 299.883 & 1.22 & 78.02 & 12.00 & 12.10 & 0.99 & 0.810588 \\
\hline 605 & 25 & 48 & 602 & 68.437 & 59 & 9677.18 & 442.782 & 1.61 & 87.42 & 10.00 & 10.70 & 0.93 & 0.579689 \\
\hline
\end{tabular}


Count of failures before adequate sample

\begin{tabular}{|c|c|c|c|c|c|c|}
\hline StdOrder & RunOrder & Time & $\begin{array}{l}\text { Temp } \\
\text { coded }\end{array}$ & Alloy & Temp & Count of failures \\
\hline 1 & 2 & $15 \mathrm{Min}$ & High & A356 & 595 & 0 \\
\hline 10 & 3 & $25 \mathrm{Min}$ & High & THX540 & 605 & 0 \\
\hline 30 & 4 & $20 \mathrm{Min}$ & High & THX540 & 605 & 1 \\
\hline 35 & 5 & $25 \mathrm{Min}$ & Low & A356 & 585 & 0 \\
\hline 18 & 6 & $20 \mathrm{Min}$ & High & THX540 & 605 & 2 \\
\hline 6 & 7 & $20 \mathrm{Min}$ & High & THX540 & 605 & 7 \\
\hline 13 & 11 & $15 \mathrm{Min}$ & High & A356 & 595 & 0 \\
\hline 43 & 12 & $20 \mathrm{Min}$ & Low & A356 & 585 & 0 \\
\hline 29 & 13 & $20 \mathrm{Min}$ & High & A356 & 595 & 1 \\
\hline 25 & 14 & $15 \mathrm{Min}$ & High & A356 & 595 & 2 \\
\hline 2 & 15 & $15 \mathrm{Min}$ & High & THX540 & 605 & 3 \\
\hline 42 & 16 & $20 \mathrm{Min}$ & High & THX540 & 605 & 0 \\
\hline 3 & 18 & $15 \mathrm{Min}$ & Low & A356 & 585 & 0 \\
\hline 34 & 19 & $25 \mathrm{Min}$ & High & THX540 & 605 & 1 \\
\hline 11 & 20 & $25 \mathrm{Min}$ & Low & A356 & 585 & 2 \\
\hline 38 & 21 & $15 \mathrm{Min}$ & High & THX540 & 605 & 4 \\
\hline 26 & 22 & $15 \mathrm{Min}$ & High & THX540 & 605 & 0 \\
\hline 27 & 23 & $15 \mathrm{Min}$ & Low & A356 & 585 & 0 \\
\hline 15 & 24 & $15 \mathrm{Min}$ & Low & A356 & 585 & 10 \\
\hline 23 & 25 & $25 \mathrm{Min}$ & Low & A356 & 585 & 0 \\
\hline 19 & 26 & $20 \mathrm{Min}$ & Low & A356 & 585 & 0 \\
\hline 7 & 28 & $20 \mathrm{Min}$ & Low & A356 & 585 & 0 \\
\hline 46 & 29 & $25 \mathrm{Min}$ & High & THX540 & 605 & 0 \\
\hline 14 & 30 & $15 \mathrm{Min}$ & High & THX540 & 605 & 0 \\
\hline 39 & 31 & $15 \mathrm{Min}$ & Low & A356 & 585 & 0 \\
\hline 31 & 32 & $20 \mathrm{Min}$ & Low & A356 & 585 & 0 \\
\hline 41 & 33 & $20 \mathrm{Min}$ & High & A356 & 595 & 0 \\
\hline 47 & 34 & $25 \mathrm{Min}$ & Low & A356 & 585 & 0 \\
\hline 33 & 35 & $25 \mathrm{Min}$ & High & A356 & 595 & 0 \\
\hline 9 & 36 & $25 \mathrm{Min}$ & High & A356 & 595 & 0 \\
\hline 21 & 37 & $25 \mathrm{Min}$ & High & A356 & 595 & 0 \\
\hline 17 & 41 & $20 \mathrm{Min}$ & High & A356 & 595 & 9 \\
\hline 37 & 43 & $15 \mathrm{Min}$ & High & A356 & 595 & 0 \\
\hline 45 & 44 & $25 \mathrm{Min}$ & High & A356 & 595 & 0 \\
\hline 5 & 45 & $20 \mathrm{Min}$ & High & A356 & 595 & 0 \\
\hline 22 & 48 & $25 \mathrm{Min}$ & High & THX540 & 605 & 0 \\
\hline
\end{tabular}




\section{Verification of failures based on alloy (result no significant effect):}

General Linear Model: Count of failures versus Alloy

Factor Information

Factor Type Levels Values

Alloy Fixed 2 A356; THX540

Analysis of Variance

Source DF Adj SS Adj MS F-Value P-Value

$\begin{array}{llllll}\text { Alloy } & 1 & 2.000 & 2.000 & 0.31 & 0.581\end{array}$

Error $\quad 34 \quad 219.0006 .441$

Total $\quad 35 \quad 221.000$

Model Summary

S R-sq R-sq(adj) R-sq(pred)

$2.53795 \quad 0.90 \% \quad 0.00 \% \quad 0.00 \%$

\section{Verification of failures based on time and temperature for A356 (result no significant effect):}

General Linear Model: Count of failures versus Temp coded; Time

Factor Information

Factor

Type Levels Values

Temp coded

Fixed 2

Time

Fixed 3

High; Low

15 Min; 20 Min; 25 Min

Analysis of Variance

$\begin{array}{llllll}\text { Source } & \text { DF } & \text { Adj SS } & \text { Adj MS } & \text { F-Value } & \text { P-Value } \\ \text { Temp coded } & 1 & 0.000 & 0.0000 & 0.00 & 1.000 \\ \text { Time } & 2 & 7.000 & 3.5000 & 0.46 & 0.641 \\ \text { Temp coded*Time } & 2 & 21.000 & 10.5000 & 1.37 & 0.279 \\ \text { Error } & 18 & 138.000 & 7.6667 & & \\ \text { Total } & 23 & 166.000 & & & \\ \text { Model Summary } & & & & & \\ \text { S } & \text { R-sq } & \text { R-sq(adj) } & \text { R-sq(pred) } & & \\ 2.76887 & 16.87 \% & 0.00 \% & 0.00 \% & & \end{array}$


Verification of failures based on time and temperature for THIXALLOY (result no significant effect):

General Linear Model: Count of failures versus Time

Factor Information

$\begin{array}{llll}\text { Factor } & \text { Type } & \text { Levels } & \text { Values } \\ \text { Time } & \text { Fixed } & 3 & \text { 15 Min; 20 Min; 25 Min }\end{array}$

Analysis of Variance

$\begin{array}{llllll}\text { Source } & \text { DF } & \text { Adj SS } & \text { Adj MS } & \text { F-Value } & \text { P-Value } \\ \quad \text { Time } & 2 & 10.50 & 5.250 & 1.11 & 0.370 \\ \text { Error } & 9 & 42.50 & 4.722 & & \\ \text { Total } & 11 & 53.00 & & & \\ & & & & & \\ \text { Model Summary } & & & & \\ \text { S } & \text { R-sq } & \text { R-sq(adj) } & \text { R-sq(pred) } & \\ 2.17307 & 19.81 \% 1.99 \% & 0.00 \% & \end{array}$




\section{Aging experiment}

\section{Design of Experiment}

\begin{tabular}{|r|r|r|r|l|l|l|r|}
\hline StdOrder & RunOrder & PtType & Blocks & Time & Temp & Alloy & Temp \\
\hline 5 & 1 & 1 & 1 & 40 Min & High & A356 & 595 \\
\hline 21 & 2 & 1 & 1 & 50 Min & High & A356 & 595 \\
\hline 15 & 3 & 1 & 1 & 30 Min & Low & A356 & 585 \\
\hline 17 & 4 & 1 & 1 & 40 Min & High & A356 & 595 \\
\hline 13 & 5 & 1 & 1 & 30 Min & High & A356 & 595 \\
\hline 8 & 6 & 1 & 1 & 40 Min & Low & THX540 & 595 \\
\hline 4 & 7 & 1 & 1 & 30 Min & Low & THX540 & 595 \\
\hline 23 & 8 & 1 & 1 & 50 Min & Low & A356 & 585 \\
\hline 20 & 9 & 1 & 1 & 40 Min & Low & THX540 & 595 \\
\hline 14 & 10 & 1 & 1 & 30 Min & High & THX540 & 605 \\
\hline 3 & 11 & 1 & 1 & 30 Min & Low & A356 & 585 \\
\hline 10 & 12 & 1 & 1 & 50 Min & High & THX540 & 605 \\
\hline 24 & 13 & 1 & 1 & 50 Min & Low & THX540 & 595 \\
\hline 19 & 14 & 1 & 1 & 40 Min & Low & A356 & 585 \\
\hline 22 & 15 & 1 & 1 & 50 Min & High & THX540 & 605 \\
\hline 7 & 16 & 1 & 1 & 40 Min & Low & A356 & 585 \\
\hline 1 & 17 & 1 & 1 & 30 Min & High & A356 & 595 \\
\hline 9 & 18 & 1 & 1 & 50 Min & High & A356 & 595 \\
\hline 11 & 19 & 1 & 1 & 50 Min & Low & A356 & 585 \\
\hline 12 & 20 & 1 & 1 & 50 Min & Low & THX540 & 595 \\
\hline 6 & 21 & 1 & 1 & 40 Min & High & THX540 & 605 \\
\hline 16 & 22 & 1 & 1 & 30 Min & Low & THX540 & 595 \\
\hline 2 & 23 & 1 & 1 & 30 Min & High & THX540 & 605 \\
\hline 18 & 24 & 1 & 1 & 40 Min & High & THX540 & 605 \\
\hline
\end{tabular}




\section{Extrusion samples A356/THIXALLOY 630}

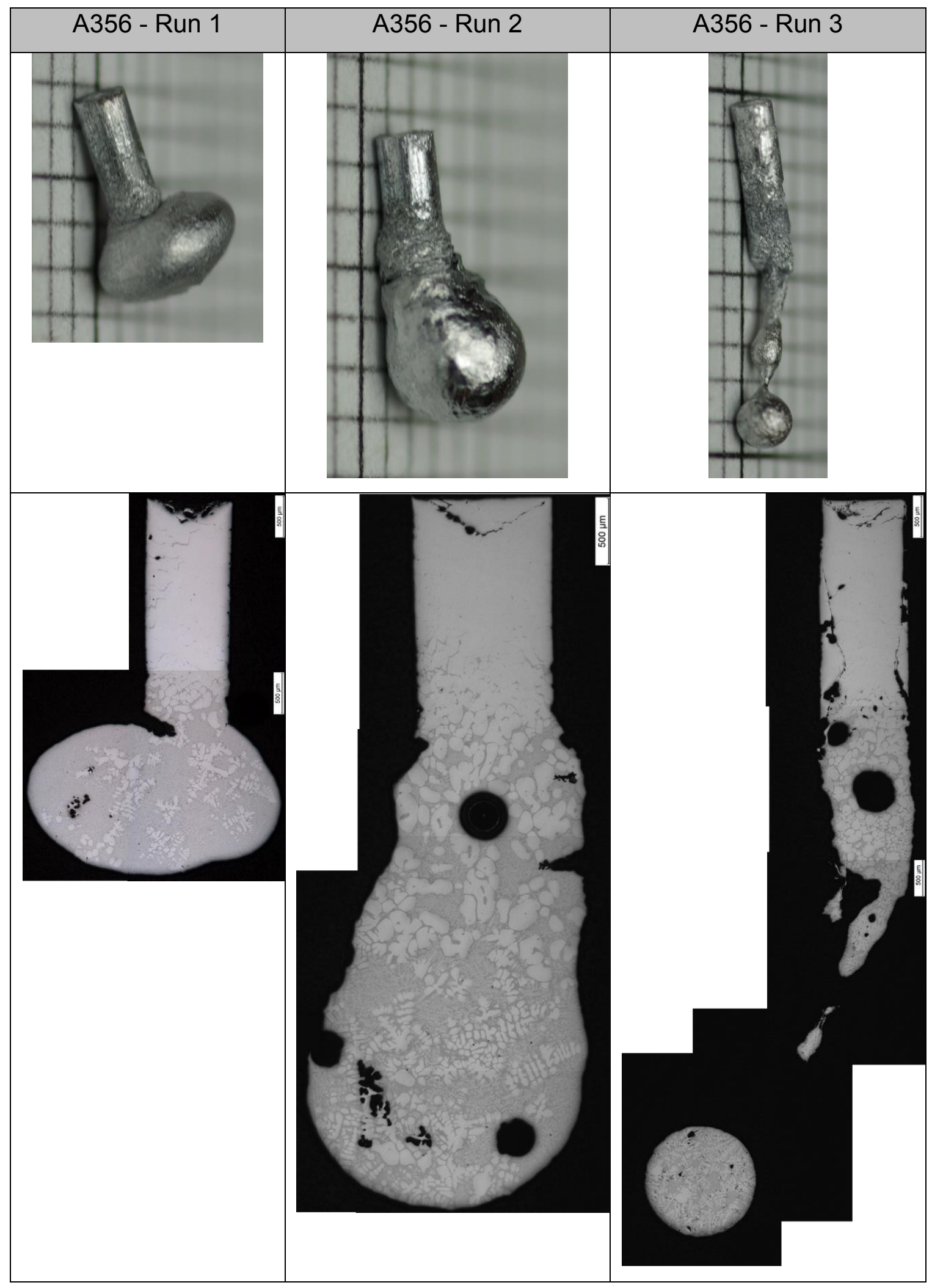




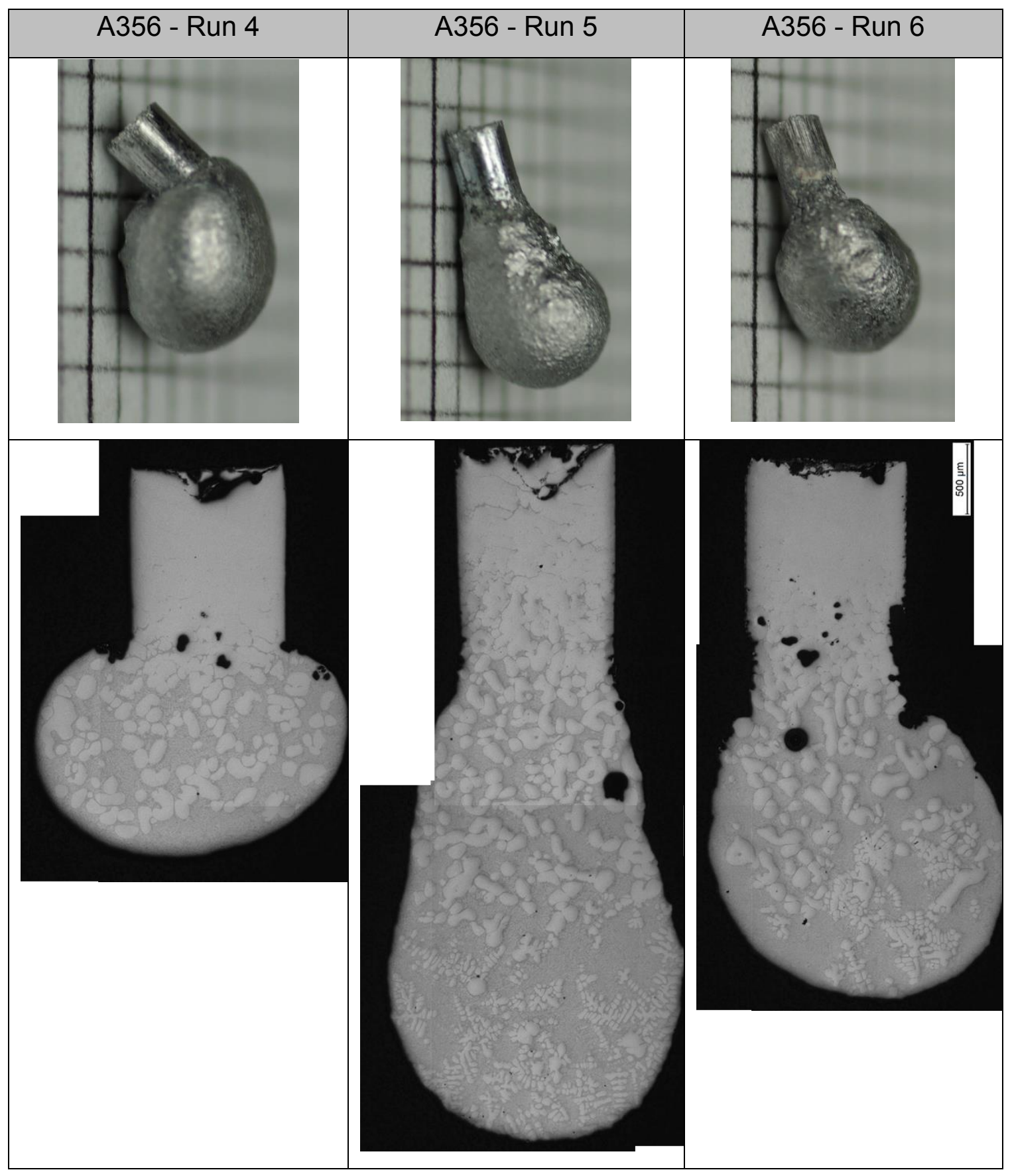




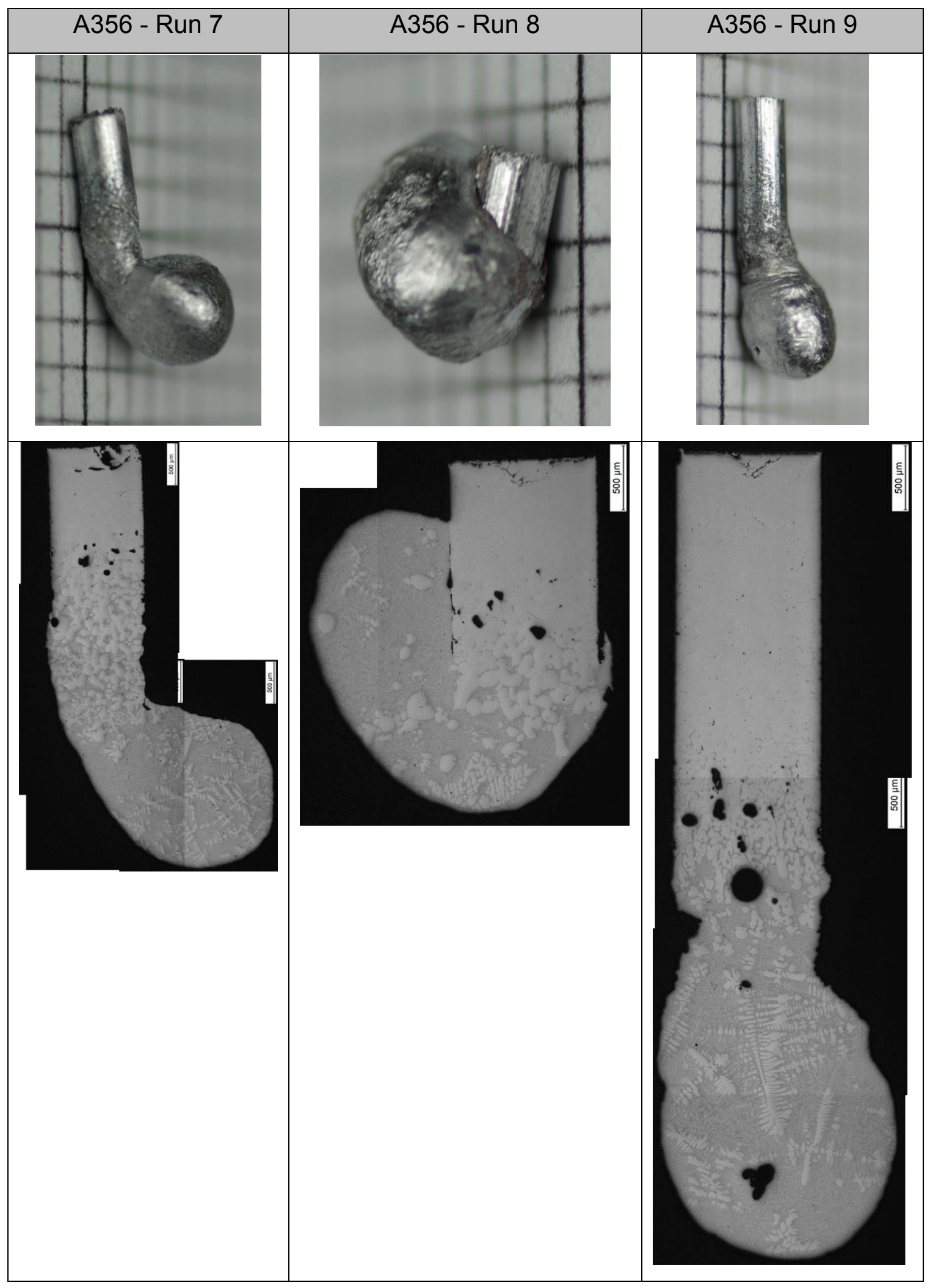




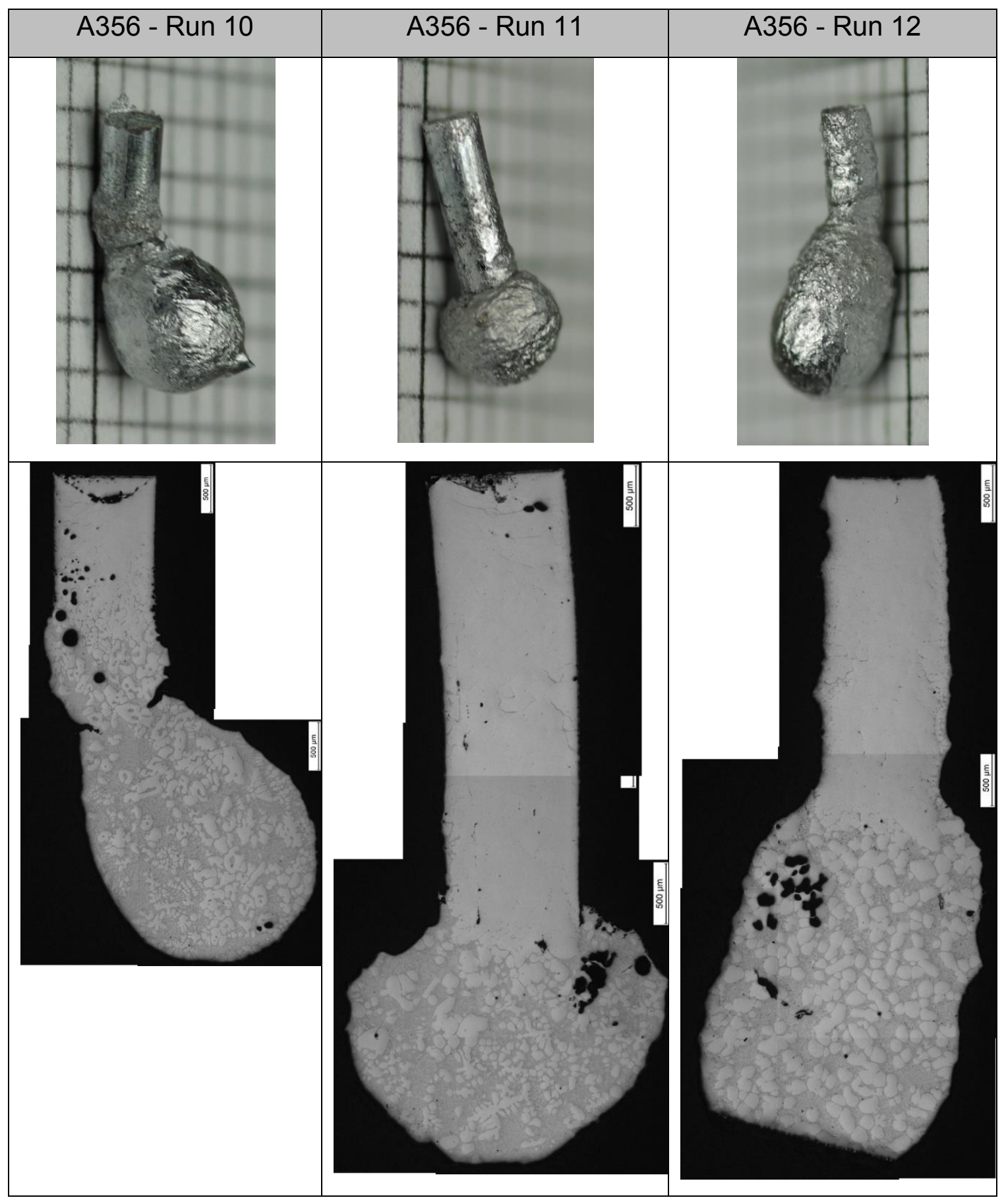


Extrusion samples THIXALLOY 540

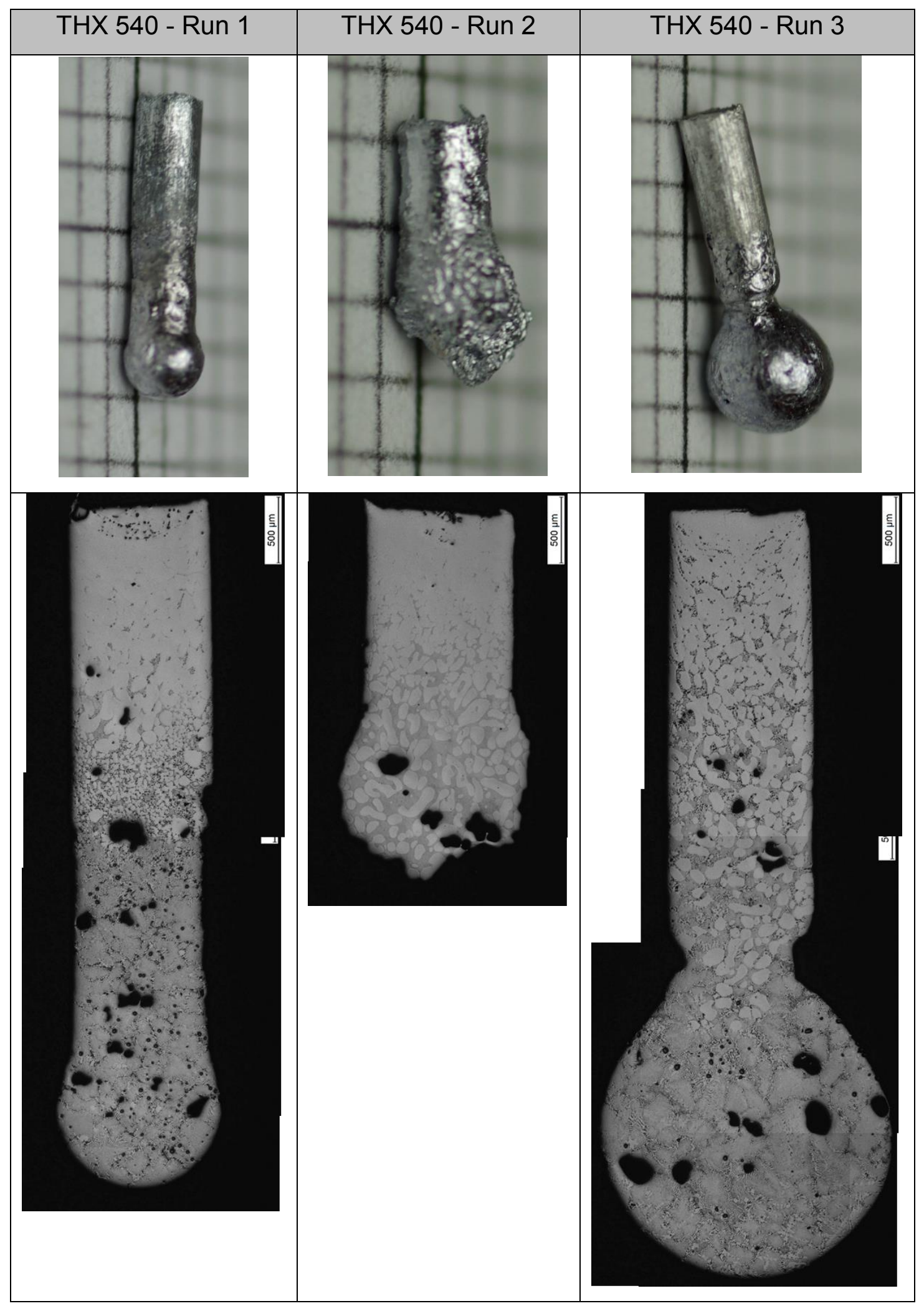




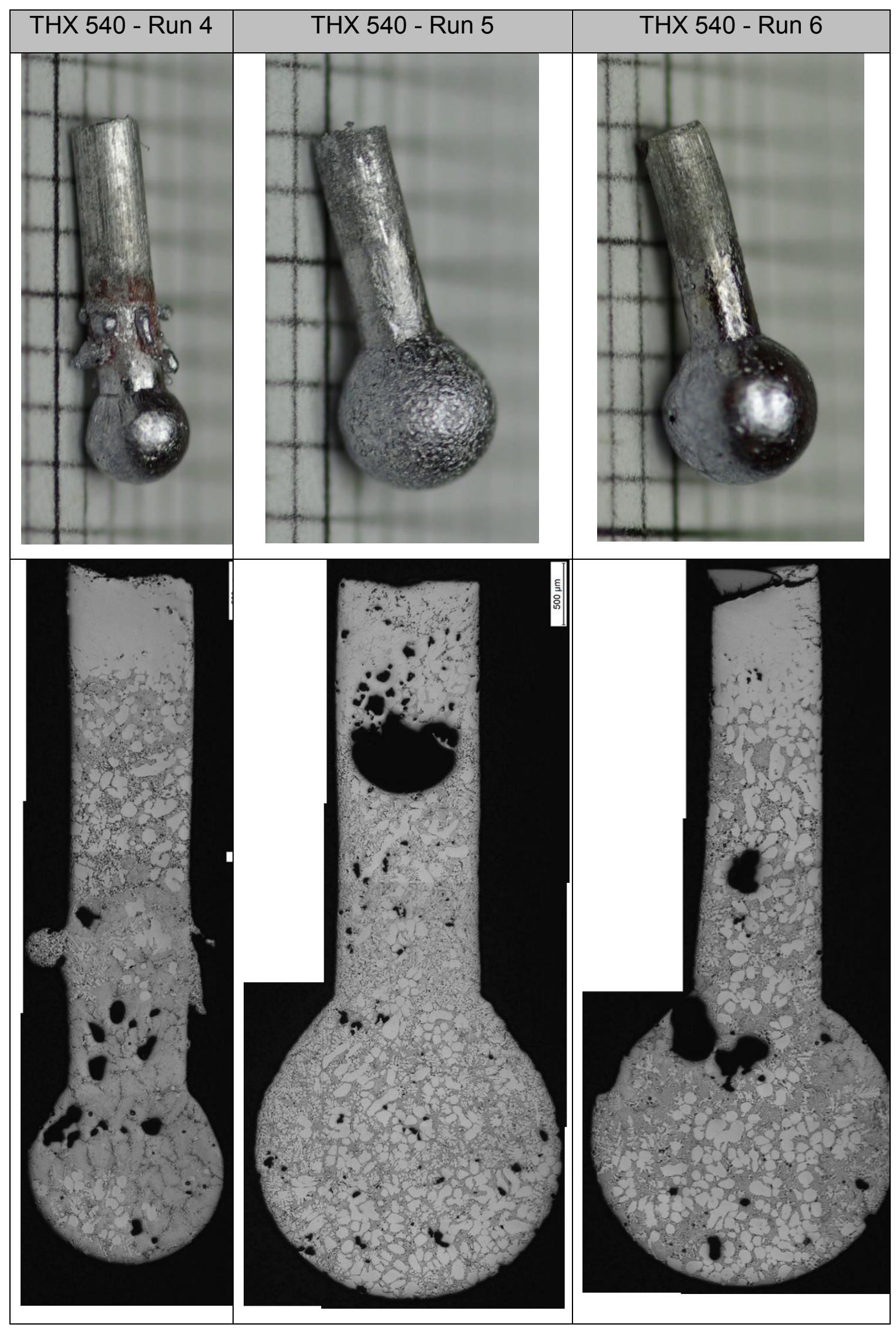




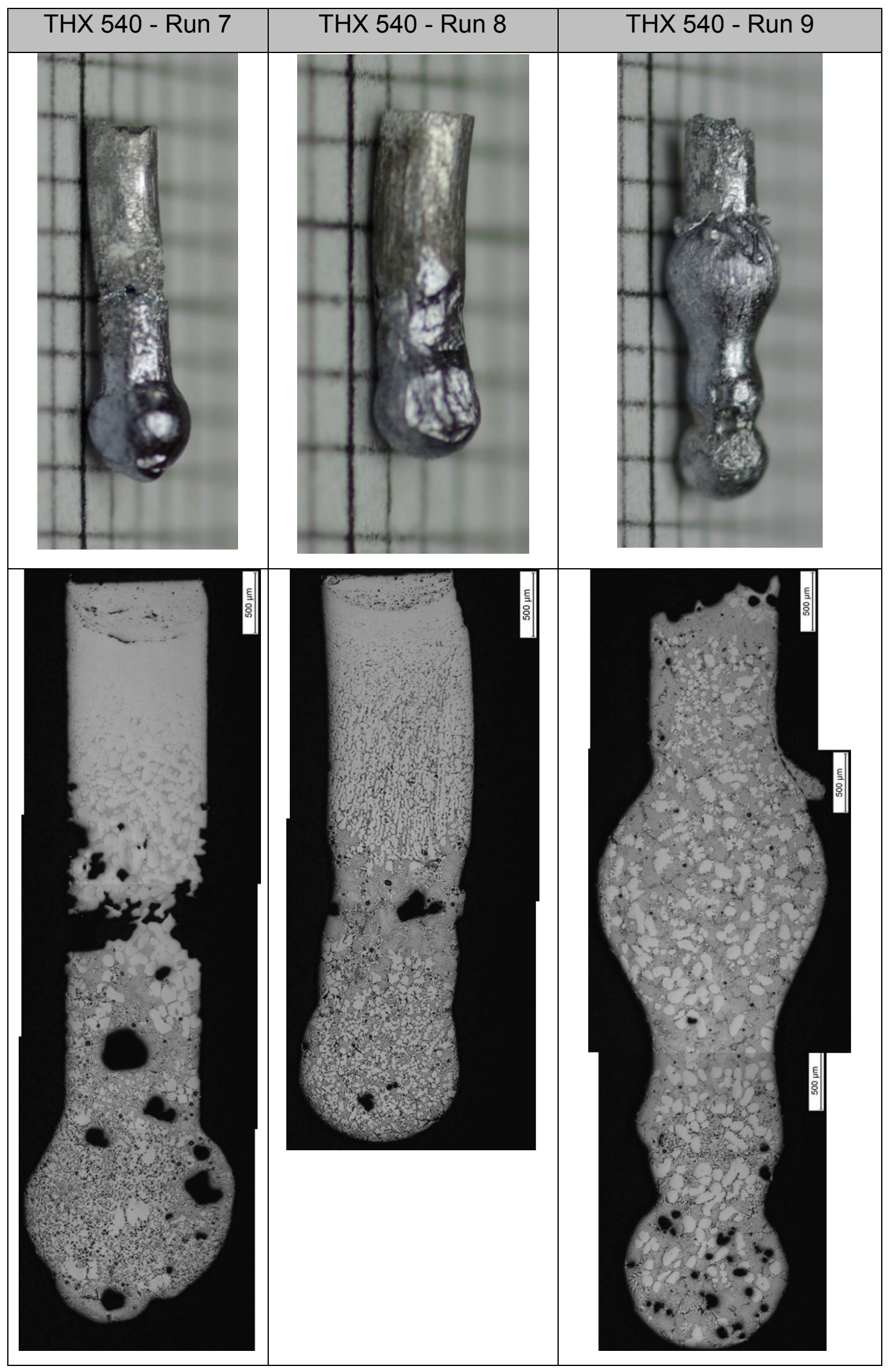




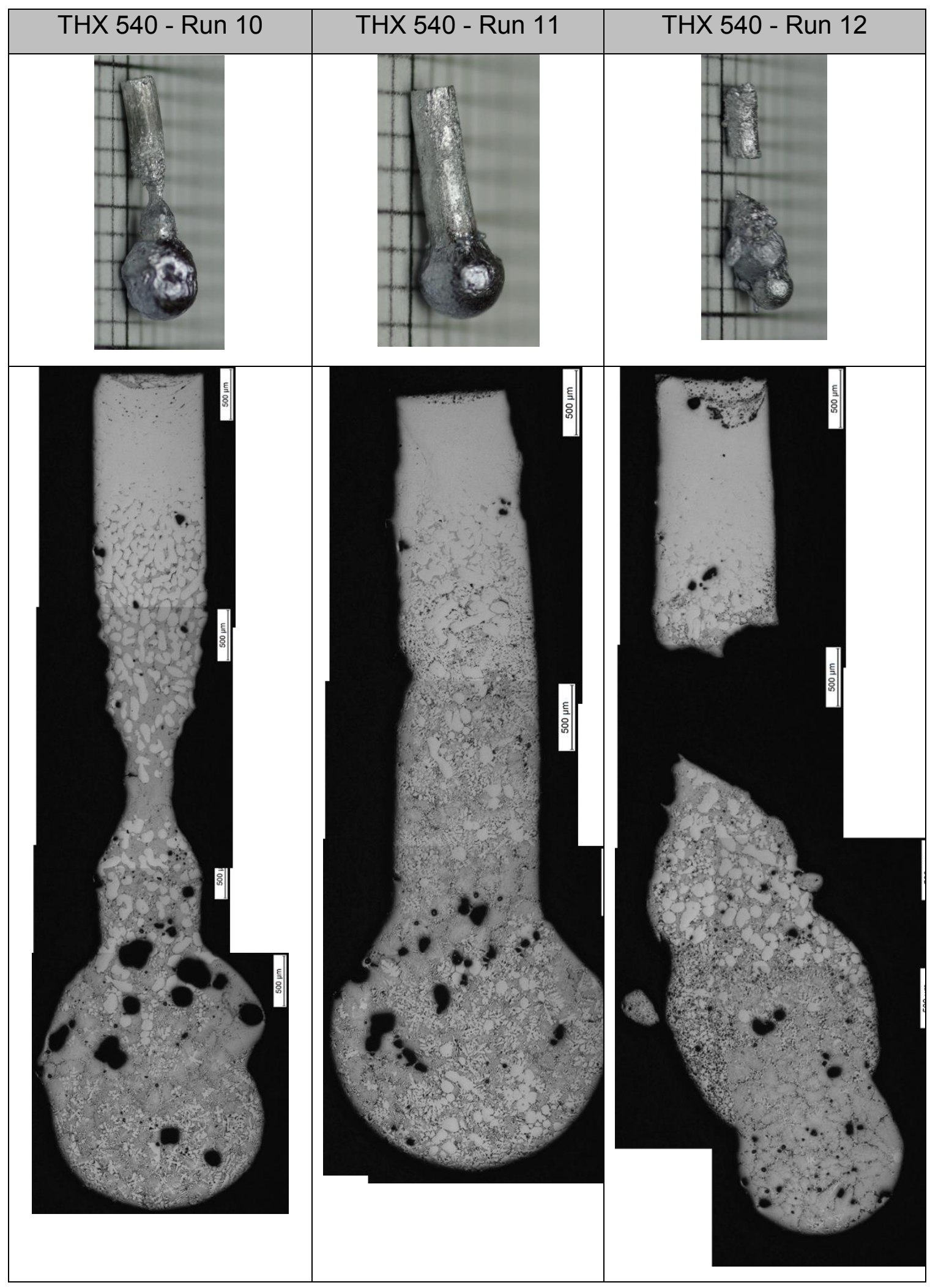




\title{
CURRICULUM VITA
}

\author{
NAME: \\ LarsHerhold \\ ADDRESS: $\quad$ Bleckmannweg 1 \\ 10367 Berlin \\ Germany \\ DOB: \\ Berlin, Germany, September 26, 1975 \\ EDUCATION \\ Dipl.Ing.(FH) Computer Engineering \\ \& TRAINING \\ University of Applied Science Berlin, Germany \\ $1997-2001$ \\ Master of Science Industrial Engineering \\ University of Louisville, KY, USA \\ 2009 - 2011 \\ PhD Student \\ University of Louisville, KY, USA \\ $2009-2011$ \\ PROFESSIONAL Information Systems Audit and Control Association (ISACA) \\ SOCIETIES International Society for Pharmaceutical Engineering (ISPE)
}

